\title{
Methodische Ansätze zur Erfassung von Waldbäumen mittels digitaler Luftbildauswertung
}

\author{
Dissertation \\ zur Erlangung des Doktorgrades \\ der Fakultät für Forstwissenschaften und Waldökologie \\ der Georg-August-Universität Göttingen
}

\author{
vorgelegt von \\ Hans-Jörg Fuchs \\ geboren in Osterode am Harz
}

Göttingen im November 2003 
D7

1. Berichterstatter: Prof. Dr. Ch. Kleinn

2. Berichterstatter: Prof. Dr. J. Saborowski

Tag der mündlichen Prüfung: 13.11.2003

erschienen als elektronisches Dokument

(http://webdoc.sub.gwdg.de/diss/2004/fuchs/index.html) 


\section{Vorwort}

Die vorliegende Arbeit entstand im Rahmen meiner Tätigkeit als wissenschaftlicher Assistent am Institut für Waldinventur und Waldwachstum der Universität Göttingen. Mein besonderer Dank gilt Herrn Prof. Dr. A. Akça, der mir die Möglichkeit gegeben hat, diese Arbeit zu schreiben. Eine Bearbeitung der Themenstellung wäre ohne seine Erfahrung und Hilfe nicht möglich gewesen.

Herrn Prof. Dr. Ch. Kleinn danke ich für die Weiterführung der Betreuung, das großzügige Gewähren von Freiräumen, die gute Zusammenarbeit und Ermutigung, die für den Abschluß dieser Arbeit unerläßlich waren.

Ich bedanke mich bei Herrn Prof. Dr. J. Saborowski für die unkomplizierte Übernahme des Koreferates und die wertvolle fachliche Hilfe.

Herrn Prof. Dr. K. von Gadow danke ich für die freundliche Arbeitsatmosphäre am Institut, die zahlreichen internationalen Begegnungen und Anregungen.

Mein Dank gilt auch der Forstlichen Versuchsanstalt in Rheinland-Pfalz und der Landesanstalt für Ökologie, Bodenordnung und Forsten / Landesamt für Agrarordnung (LÖBF / LAfAO) in Nordrhein-Westfalen, die durch finanzielle Förderung, Bereitstellung der Luftbilder und Grundlagendaten die vorliegenden Untersuchungen ermöglichten.

Mein Dank gehört Dr. Hyun-Kook Cho, Stefan Kunth, Mike Kahle, Hendrik Heydecke und Bettina Wegner für ihre engagierte Zusammenarbeit in mehreren Forschungsprojekten und Ulrike Dockter für ihre spontane Einsatzbereitschaft.

Kai Böttcher vom Landesamt für Forsten und Großschutzgebiete in MecklenburgVorpommern und den MitarbeiterInnen am Institut danke ich für die freundschaftliche Unterstützung und fachlichen Diskussionen. Axel Buschmann, Stefan Kunth sowie meiner Frau Birgit danke ich für die Hilfe beim Korrigieren der Arbeit.

Voraussetzung zur Realisierung vieler Ideen war die Möglichkeit der Verwendung von Computer-Programmen, die der Freien Software-Lizenz des GNU-Projektes unterliegen. Besonders danke ich den ProgrammiererInnen und aktiven HelferInnen des Geoinformationssystemes GRASS, der Datenbank PostgreSQL und des StatistikSystemes R, die mich zu neuen Erkenntnissen geführt haben. 



\section{Inhaltsverzeichnis}

$\begin{array}{lr}\text { Inhaltsverzeichnis } & \mathbf{5}\end{array}$

1 Einleitung $\quad 7$

1.1 Zielsetzung ....................... 8

1.2 Waldzustandserfassung in Europa . . . . . . . . . . . . . . . 9

1.3 Einsatz der Fernerkundung auf Dauerbeobachtungsflächen . . . . . . . 12

2 Literaturübersicht 14

2.1 Erkennung von Waldschäden mit Farbinfrarot-Luftbildern . . . . . . . 14

2.2 Waldwachstumskundliche Auswertungen und Luftbildanwendungen . . 16

3 Material und Methoden $\quad 18$

3.1 Untersuchungsflächen . . . . . . . . . . . . . . . . . . . . 18

3.2 Datengrundlage und Bildmaterial . . . . . . . . . . . . . . . . 19

3.2 .1 Luftbilder . . . . . . . . . . . . . . . . . . . . 19

3.2.2 Terrestrische Erhebungen . . . . . . . . . . . . . 23

3.2.2.1 Kronenverlichtung und -vergilbung . . . . . . . 23

3.2.2.2 Dendrometrische Daten . . . . . . . . . . . 24

3.3 Untersuchungsmethoden . . . . . . . . . . . . . . . . . 24

3.3.1 Relevante Eigenschaften von Luftbildaufnahmen . . . . . . . . 24

3.3.1.1 Reflexion von Waldbäumen und Waldbeständen . . . 24

3.3.1.2 Atmosphärische Einflüsse . . . . . . . . . . . . 28

3.3.1.3 Sonnen- und Beobachtungsgeometrie . . . . . . . . 28

3.3.2 Technische Grundlagen der Luftbildauswertung . . . . . . . . . 29

3.3.2.1 Analytischer Stereoplotter . . . . . . . . . . . . . 29

3.3.2.2 Bildorientierung . . . . . . . . . . . 32

3.3.2.3 Scanner . . . . . . . . . . . . . 35

3.3.2.4 Digitale Fotogrammetrische Systeme (DFS) . . . . . 35

3.3.2.5 Digitale Höhenmodelle . . . . . . . . . . . . . . . 36

3.3.2.6 Digitale Orthofoto-Herstellung . . . . . . . . . . 39

3.3.2.7 Datenverarbeitung . . . . . . . . . . . . . 39

3.3 .3 Luftbildmerkmale . . . . . . . . . . . . . . . . 41

3.3.3.1 Interpretationsschlüssel . . . . . . . . . . 42

3.3.3.2 Segmentierung . . . . . . . . . . . . 43

3.3.3.3 Spektrale Merkmale . . . . . . . . . . . . . . 46

3.3.3.4 Formmerkmale . . . . . . . . . . . . . . . 48 
3.3.3.5 Baumhöhe und Höhenänderung . . . . . . . . . . 56

3.3.3.6 Überschirmungsgrad . . . . . . . . . . . . . . . 56

3.3.3.7 Stehendes Totholz . . . . . . . . . . . . 57

3.3.3.8 Erkennung von ausgeschiedenen Bäumen . . . . . 58

3.3.4 Ablauf der Luftbildauswertung . . . . . . . . . . . . . . . 59

3.3.4.1 Visuelles Auswertungsverfahren . . . . . . . . . 60

3.3.4.2 Digitales Auswertungsverfahren . . . . . . . . . 62

3.3.5 Genauigkeit digitaler Höhenmodelle . . . . . . . . . . . . . . 62

3.3.6 Statistische Auswertung ................. 65

3.3.6.1 Geometrische Genauigkeit . . . . . . . . 65

3.3.6.2 Thematische Genauigkeit . . . . . . . . . . 66

3.3.6.3 Klassifikation . . . . . . . . . . . . 67

3.3.6.4 Lineare Regressionsanalyse . . . . . . . . . . . 69

4 Ergebnisse $\quad \mathbf{7 1}$

4.1 Bewertung der automatischen Erstellung von Höhenmodellen . . . . . . 71

4.1.1 Vergleich mit terrestrischen Baumhöhen . . . . . . . . . . . 71

4.1.2 Vergleich mit Höhen aus einem analytischen Stereoplotter . . . 74

4.1.3 Vergleich von Höhendifferenzen im dreifachen Überdeckungsbereich von Stereomodellen . . . . . . . . . . . . . 81

4.2 Überschirmungsgrad . . . . . . . . . . . . . . . . . . . . . . 82

4.3 Segmentierung . . . . . . . . . . . . . . 86

4.4 Farb- und Formmerkmale von Baumkronen . . . . . . . . . . . . . . . 88

4.4.1 Farbmerkmale im digitalen Orthofoto . . . . . . . . . . . . . 88

4.4.2 Kronenbreite, -länge, -schirmfläche, -mantelfläche und -volumen 91

4.4.3 Kompaktheit, Länglichkeit und Orientierung . . . . . . . . . 95

4.4.4 Kronenrauhigkeit und Gaußsche Krümmung . . . . . . . . . . 99

4.5 Erkennung von ausgeschiedenen Bäumen _ . . . . . . . . . . . 101

4.6 Baumart . . . . . . . . . . . . . . . . . 103

4.7 Vitalität . . . . . . . . . . . . . . . . . . . . . 104

4.8 Beziehungen zwischen Grundflächenzuwachs und Kronenvariablen . 105

5 Diskussion $\quad 112$

$\begin{array}{lll}6 & \text { Zusammenfassung } & 118\end{array}$

$\begin{array}{ll}\text { Literaturverzeichnis } & 122\end{array}$ 


\section{Einleitung}

Fotografische Luftbildaufnahmen aus Flugzeugen sind seit Jahrzehnten wichtige Hilfsmittel zur Informationsgewinnung in der Forstwirtschaft. Luftbildaufnahmen repräsentieren ein flächenabbildendes Fernerkundungssystem. Daten und Bilder von Wäldern können aufgenommen, gespeichert und zu einem späteren Zeitpunkt ausgewertet werden, ohne dass eine Anwesenheit erforderlich ist. Zu den vielfältigen forstlichen Anwendungsbereichen gehören z. B. die Orientierung im Gelände, die Kartenerstellung und Aktualisierung von Geoinformationssystemen (GIS), die Einteilung von Waldbeständen, die Erfassung des Zustandes und der Veränderung von Wäldern im Rahmen von nationalen, regionalen und betrieblichen Inventuren und die Überwachung von Waldschäden.

Obwohl in den letzten Jahren neue digitale Fernerkundungssensoren entwickelt wurden, werden Reihenmeßkammern und Luftbildfilme in aktuellen Befliegungen für praktische Anwendungen bevorzugt. Die Gründe liegen in der ausgereiften Technik und der hohen geometrischen Auflösung und Genauigkeit. Außerdem existieren große öffentlich zugängliche Luftbildarchive, die einen unschätzbaren Wert für die Erfassung der Veränderung der Landoberfläche haben.

Analoge Luftbilder wurden bisher überwiegend mit visuellen Methoden der Bildinterpretation und Bildmessung (Fotogrammetrie) ausgewertet. Seit Mitte der 80er Jahre gewinnt die Entwicklung der digitalen Fotogrammetrie an Bedeutung. Analoge Luftbilder können mit einem Scanner in ein digitales Format überführt werden, was die quantitative Analyse mit digitalen Bildverarbeitungsmethoden ermöglicht. Die zunehmende Prozessorleistung und Speicherkapazität der Computer erleichtert die Informationsgewinnung aus Luftbildern. Die Technik der computergesteuerten Stereoplotter (analytische Auswertungsgeräte) wird durch Software auf kostengünstigen StandardComputern ersetzt (Heipke 2001, Jacobsen 2001). Erst durch diese Technik erhalten viele Fachbereiche einen Zugang zur intensiven Nutzung von Luftbildern. Weitere Vorzüge der digitalen Fotogrammetrie sind die Automatisierung der Bildorientierung und Erstellung von Oberflächenmodellen. Digital entzerrte Fotos (Orthofotos) können in Geoinformationssysteme integriert werden und bieten neue Möglichkeiten der Bildanalyse.

Der Informationsbedarf über Wälder ist gestiegen, weil die Waldbewirtschaftung nicht nur auf die Maximierung der Holzproduktion ausgerichtet ist, sondern gleichzeitig die Stabilität von Waldökosystemen berücksichtigt (Pretzsch 2002). Ein Beispiel für die Erweiterung des Informationsinteresses ist die Waldzustandserfassung in $\mathrm{Eu}-$ ropa. Sie beruht auf Stichprobenerhebungen auf einem permanent eingerichteten Netz von Probeflächen und auf der Anlage langfristiger Versuchsflächen. 
In terrestrischen Wiederholungsinventuren wird eine Vielzahl von Variablen erhoben, die unter anderem wesentliche Kenngrößen der Vitalität ${ }^{1}$ von Wäldern beschreiben. Daneben werden Luftbilder zur Dokumentation der langfristigen Versuchsflächen (Dauerbeobachtungsflächen des Level 2-Programmes) eingesetzt. Die Arbeitsgruppe „Remote Sensing for Forest Health Assessment" der Europäischen Union (EU) empfiehlt ausdrücklich den Einsatz von großmaßstäbigen Farbinfrarot-Luftbildern. Merkmale und Methoden zur Auswertung der Luftbilder sind in einem EU-Handbuch dokumentiert (WGRSA 2000). Es werden fünf Intensitätsstufen der Verwendung von Luftbildern im Level 2-Programm unterschieden, die von der Dokumentation bis zur detaillierten fotogrammetrischen Analyse reichen.

\subsection{Zielsetzung}

Ziel der vorliegenden Arbeit ist die Zustandserfassung von acht ausgewählten Dauerbeobachtungsflächen in Rheinland-Pfalz und Nordrhein-Westfalen durch Zeitreihenauswertungen von Farbinfrarot-Luftbildern in den Maßstäben 1:2700 bis 1:4300. Die Untersuchungen konzentrieren sich auf die folgenden Fragestellungen:

1. Welche Baummerkmale können aus digitalen Farbinfrarot-Luftbildern bestimmt werden?

2. Welche Methoden eignen sich zur Ableitung von Variablen, die für die Waldzustandserfassung relevant sind?

3. Welche Beziehungen gibt es zwischen Baummerkmalen im Luftbild und dem aus terrestrischen Messungen hergeleiteten Grundflächenzuwachs?

Neben der Fotointerpretation (Deutung von Bildinhalten) werden Methoden der Fotogrammetrie (dreidimensionale Rekonstruktion von Lage und Form von Objekten aus Fotografien (Kraus 1997)) eingesetzt. Der Schwerpunkt der vorliegenden Untersuchungen liegt in Anwendungen der digitalen Fotogrammetrie.

Bei der Suche nach aussagefähigen Merkmalen ist es naheliegend, die Vorgehensweise der visuellen Interpretation darzustellen, bei der Farben, geometrische Strukturen sowie die räumliche Umgebung berücksichtigt werden. Die flächendeckende visuelle Interpretation von Einzelbäumen in Luftbildern ist zeitaufwändig und unterliegt subjektiven Einflüssen. Deshalb gibt es viele Bemühungen, die Bildinterpretation zu automatisieren und zu objektivieren. Die menschliche Fähigkeit der Objekterkennung

\footnotetext{
${ }^{1}$ Vitalität bedeutet Lebenskraft, Lebendigkeit oder Lebensfülle (Duden 2000). Wird diese Bedeutung auf Waldbäume übertragen, kann man darunter den Gesundheitszustand oder die Überlebensfähigkeit bei Stress und Beschädigungen verstehen (Roloff 1989). Vitalität ist keine direkt messbare Eigenschaft, sondern kann nur anhand verschiedener Indikatoren beurteilt werden. Beispiele solcher Indikatoren sind Holzzuwachs, Kronenverlichtung und ihre Veränderung oder auch der Längenzuwachs von Trieben (Brang 1998, S. 14). Unter Waldschäden wird im Allgemeinen eine Störung verstanden, die durch negative Folgen gekennzeichnet ist, wie die Reduktion des Wachstums und eine aktuell erhöhte Mortalität von Bäumen (Franklin 2001, S. 303).
} 
übersteigt bisher das ausschließliche Erkennen und Deuten des Bildinhaltes über Computerprogramme (Mustererkennung). Mit der Integration von Methoden der digitalen Bildverarbeitung, Mustererkennung und Geoinformatik sollen Ansätze zur Verbesserung der digitalen Luftbildauswertung erprobt werden.

Die Ermittlung und Prognose des Holzzuwachses von Bäumen ist von zentraler Bedeutung für eine nachhaltige Waldbewirtschaftung. Vom praktischen Standpunkt aus interessiert der Durchmesserzuwachs des Stammes, weil einer Durchmesserzunahme meist einer Wertzunahme entspricht. Das Wachstum eines Stammes als biologischer Prozess besteht in dem Ansetzen eines neuen Holzmantels. Deshalb wäre eine Ermittlung der Volumenzunahme zweckmäßig. Da die Erhebung des Volumenzuwachses eines stehenden Stammes aufwändig und mit größeren Fehlern behaftet ist, wird in dieser Arbeit der Grundflächenzuwachs ${ }^{2}$ als Weiser für die Holzproduktion des Einzelbaumes eingesetzt.

Der Holzzuwachs eines Baumes ist ein Indikator für die Vitalität eines Baumes. Der Durchmesser- und Grundflächenzuwachs steht im engen Zusammenhang mit der Baumkrone, in der lebenswichtige Vorgänge der Assimilation stattfinden. Die Untersuchung der Beziehungen zwischen Nadel- / Blattverlusten und dem Grundflächenzuwachs ist deshalb von Interesse. Darüber hinaus sind Kronendimensionen eine wichtige Grundlage für einzelbaumorientierte Waldwachstumsmodelle. Eine Erhebung mit Methoden der Fernerkundung könnte zur Verbesserung oder Verifizierung der Modelle beitragen.

\subsection{Waldzustandserfassung in Europa}

Ende der siebziger Jahre wurden erste Symptome einer Verschlechterung der Vitalität der Waldbäume in verschiedenen europäischen Ländern als Verlichtung und Vergilbung der Baumkronen beobachtet. In verschiedenen Kombinationen waren diese an dem Syndrom "neuartiger Waldschäden" beteiligt; viele Baumarten auf großen Flächen waren hiervon betroffen. Bei der Suche nach den Ursachen fanden sich immer mehr Hinweise, dass grenzüberschreitende Luftverunreinigungen eine maßgebliche Rolle spielen.

Die Bemühungen um eine verbesserte Beobachtung und Überwachung der "neuartigen Waldschäden" in Europa führten dazu, dass 1985 die „United Nations Economic Commission for Europe“ (UN/ECE) unter der „Convention on Long-Range Transboundary Air Pollution“ (CLRTAP) das „International Cooperative Programme on the Assessment and Monitoring of Air Pollution Effects on Forests" (ICP-FOREST) etablierte.

1986 akzeptierten die Mitgliedsstaaten der Europäischen Union (EU) das „Scheme on the Protection of Forests against Atmospheric Pollution“. Weitere internationale politische Prozesse wie die Resolution Nr. 1 der 1. „Ministeral Conference on the Protection of Forests in Europe“ (Strasbourg 1990) und Resolution Nr. 1, 2 und 4 der 2. „Mi-

\footnotetext{
${ }^{2}$ Die Grundfläche eines Baumes wird als kreisförmiger Stammquerschnitt in 1,3 m Höhe definiert.
} 
nisteral Conference on the Protection of Forests in Europe" (Helsinki 1993) beinhalten allgemeine Leitlinien für eine nachhaltige Forstwirtschaft in Europa, die Bewahrung der biologischen Diversität und langfristigen Anpassung der Wälder an Klimaveränderungen (MCPFE 1993). Seit 1994 haben ICP Forests und die Europäische Union Zielsetzungen in enger Kooperation erweitert und ein System der Umweltkontrolle mit den Intensitäts-Stufen Level 1, 2 und 3 aufgebaut. Zur Zeit nehmen 36 europäische Länder, Kanada und die USA teil. Das Monitoring-System ist nicht nur relevant für die Politik der Luftreinhaltung, sondern auch für andere internationale politische Prozesse, die Biodiversität, eine nachhaltige Forstwirtschaft und Klimaveränderungen berücksichtigen (ICP-FOREST 2000).

Die Intensitätsstufe Level 1 wird seit 1986 auf einem großräumigen transnationalen systematischen Stichprobennetz (16 x 16 km) durchgeführt. Jeder Stichprobenpunkt ist durch eine Anzahl von Variablen, wie z.B. der jährlichen Aufnahme des Kronenzustandes, gekennzeichnet. Zusätzlich werden Untersuchungen zum Boden- und Ernährungszustand der Bäume durchgeführt.

Das Hauptziel des Level 1-Programmes ist die Gewinnung von Informationen über räumliche und zeitliche Variationen des Waldzustandes in Beziehung zu Stressfaktoren (insbesondere Luftverunreinigung) auf regionaler, nationaler und internationaler Ebene.

Das "European Programme for the Intensive Monitoring of Forest Ecosystems" (Intensitätsstufe Level 2) (Dauerbeobachtungsflächenprogramm) begann 1994 mit der Einrichtung von permanenten Untersuchungsflächen mit einer Flächengröße von mindestens 0,25 ha. Es handelt sich um Fallstudien zur Langzeitüberwachung (mind. 15 - 20 Jahre), die nach den folgenden allgemeinen, nicht statistischen Kriterien ausgewählt wurden (BMELF 1994, 1997):

- Berücksichtigung der wichtigsten Baumarten und Wachstumsbedingungen des jeweiligen Landes,

- Mindestfläche 0,25 ha,

- genügende Anzahl von Bäumen für dauernde Probennahme auf oder in der Nähe der Fläche,

- kein direkter Schadstoffeintrag durch nahe gelegene, bekannte Quellen (z. B. landwirtschaftliche Betriebe, Hauptverkehrsstraßen oder Industriebetriebe),

- Einrichten einer Pufferzone, deren Breite von Alter und Typ des Waldes abhängig ist,

- Beobachtungsfläche und Pufferzone mit möglichst einheitlicher Zusammensetzung von Baumarten, Alter, Bodenart und Hangneigung,

- keine Differenzen in der Bewirtschaftung der Fläche, der Pufferzone und des umgebenden Bestandes,

- ausreichende Entfernung zu Bestandesrändern,

- permanente Markierung der Eckpunkte und Grenzen der Flächen,

- dauerhafte Nummerierung aller Probebäume, 
- keine Beschränkung hinsichtlich des Zugangs oder der Probennahme.

Bisher wurden 865 Beobachtungsflächen in 30 europäischen Ländern ausgewählt (ICPFOREST 2000). Die in der vorliegenden Arbeit untersuchten Flächen Eiche 502, Eiche 405 (Level 2-Nr. 705) und Fichte 130 - 133 (Level 2-Nr. 701) wurden in dieses Programm aufgenommen. Auf den Beobachtungsflächen werden Erfassungen des Kronenzustandes, der Wachstums- und Ernährungssituation der Bäume sowie weiterer Einflussfaktoren (Witterung, Bodenzustand, Stoffeinträge und -austräge) durchgeführt (Tab. 1.1).

Tab. 1.1: Erhebungen auf der Intensitätsstufe Level 2 (ICP-FOREST 2000)

\begin{tabular}{lll}
\hline Indikator & $\begin{array}{l}\text { zeitliche } \\
\text { Auflösung }\end{array}$ & Intensität \\
\hline Kronenzustand & jährlich & alle Flächen \\
Nadel- / Blattanalysen & alle 2 Jahre & alle Flächen \\
Bodenchem. Kapazitäts- und & alle 10 Jahre & alle Flächen \\
Intensitätsparameter & alle 5 Jahre & alle Flächen \\
Zuwachs & kontinuierlich & $>10 \%$ der \\
Atmosphärische Deposition & $>10 \%$ der \\
Chemie der Bodenlösung & kontinuierlich & Flächen \\
(Sickerwasser) & & $>10 \%$ der \\
Bodenvegetation & alle 5 Jahre & Flächen \\
& & $>10 \%$ der \\
Meteorologische Parameter & kontinuierlich & Flächen \\
Luftqualität (z. B. Ozon) & alle 1-4 Wochen & \\
Phänologie & optional & \\
Bodenfauna & optional & \\
Fernerkundung & optional & \\
\hline
\end{tabular}

Das ursprüngliche Hauptziel des Untersuchungsprogrammes Level 2 ist ein besseres Verständnis der Auswirkungen der Luftverunreinigung (besonders durch Schwefel- und Stickoxide, Ammoniak, Ammonium) und anderer Stressfaktoren auf Waldökosysteme. Weitere Ziele sind: 
- Beschreibung des Zustandes und der natürlichen und anthropogen bedingten Veränderungen von Waldökosystemen,

- Erhebung von verlässlichen raumbezogenen und zeitlich hochauflösenden Informationen unter Berücksichtigung heutiger und zukünftig zu erwartender Stoffeinträge sowie klimatischer Veränderungen,

- Aufbau einer Datengrundlage und Infrastruktur für Grundlagenforschung und angewandte Fragestellungen,

- Grundlagen für heutige und weit in die Zukunft reichende Behandlungs- und Pflegekonzepte der forstlichen Praxis,

- Bereitstellung von Informationen und Ergebnissen, die auf einen europäischen Maßstab übertragen werden können.

Einige Untersuchungen (Kronenzustandsbeurteilung, Nadel- / Blattanalysen, chemische Bodenanalyse) werden auf der Level 1 und Level 2-Stufe gleichzeitig durchgeführt. Hierdurch sollen Ergebnisse auf europäische Länderebene übertragen werden.

Die Intensitätsstufe Level 3 sieht nicht die Einrichtung neuer Untersuchungsflächen vor, sondern die Zusammenführung von Informationen aus Level 1 und 2 (BMELF 1994, 1997).

\subsection{Einsatz der Fernerkundung auf Dauerbeobachtungsflächen}

Die langfristige Auslegung des Level 2-Programmes erfordert die exakte Darstellung von Auswahlkriterien und Methoden, die für die Beschreibung des Waldzustandes und seiner Veränderungen angewendet werden können. Da die Erhebungen von verschiedenen Ländern und Institutionen durchgeführt werden, ist die Erarbeitung und Anwendung objektiver und reproduzierbarer Verfahren eine notwendige Voraussetzung. Standardverfahren existieren bisher nur für terrestrische Erhebungen (ICP-FOREST 1994).

Der Einsatz von Methoden der Fernerkundung auf der Level 2-Ebene ist optional und eine von vielen Möglichkeiten, die zur Überwachung des Gesundheitszustandes der Wälder angewendet werden können. In Deutschland, Frankreich, Italien, Irland und Österreich liegen für einige Level 2-Flächen mehrjährige CIR-Luftbildzeitreihen vor, die in Deutschland teilweise bis in die 80er Jahre zurückreichen. Empfehlungen zur Auswertung der CIR-Luftbilder (WGRSA 2000, Wolff et al. 1999) sehen fünf Intensitätsstufen vor:

1. Dokumentation ohne weitere Auswertungen,

2. wie Option 1 und Erhebung der Repräsentanz einer Untersuchungsfläche durch visuelle Interpretation der näheren Umgebung,

3. wie Option 2 und visuelle Interpretation von Einzelbaummerkmalen,

4. wie Option 3 und fotogrammetrische Messungen von Einzelbäumen,

5. wie Option 4 und fotogrammetrische Modellierung von Gelände- und Kronenoberflächenmodellen. 
Die Auswertungsverfahren beruhen überwiegend auf Methoden der visuellen Fotointerpretation mit Hilfe einfacher Auswertungsgeräte. Erst mit den Optionen 4 und 5 ist die Verwendung fotogrammetrischer Instrumente vorgesehen. Methoden der digitalen Bildverarbeitung werden bisher nur für die Auswertung von digitalen Satellitenbildern vorgeschlagen. In dem EU-Projekt SEMEFOR (Satellite Based Environmental Monitoring of European Forests) werden die Einsatzmöglichkeiten von satellitengetragenen Sensoren für das Level 1-Monitoring in Europa untersucht. Ein Ergebnis dieser Studie ist der Vorschlag, die Level 2-Flächen als Grundlage für die Klassifikation der Satellitenszenen zu verwenden (SEMEFOR 2002). 


\section{Literaturübersicht}

\subsection{Erkennung von Waldschäden mit Farbinfrarot-Luftbildern}

Die Bedeutung des Einsatzes der Fernerkundung zur Erkennung von Schäden in der Land- und Forstwirtschaft spiegelt sich in einer großen Anzahl von Veröffentlichungen wieder. Ein umfassender Überblick wird in den Darstellungen von Henninger und Hildebrandt (1980), Hildebrandt (1996) und Pröbsting (1996) gegeben. Im Folgenden wird auf einige wichtige Arbeiten eingegangen, die für die Auswertung von CIR-Luftbildern von Bedeutung sind.

Luftbilder werden seit Jahrzehnten erfolgreich zur Erkennung von Waldschäden genutzt. In den USA und Kanada wurden bereits in den 60er und 70er Jahren visuelle Bildinterpretationen zur Erfassung von Insektenschäden eingesetzt (Murtha 1972, Ciesla 1974). Waldschäden in der Nähe von Industrie- und Rauchfeuerungsanlagen konnten in Europa nachgewiesen werden (Hildebrandt und Kenneweg 1968, Wolf 1970, Kenneweg 1972).

Luftbildgestützte Inventuren kamen in den 80er Jahren großflächig in Europa zum Einsatz. Eine Pilotstudie zur großräumigen Erfassung emissionsbedingter Waldschäden wurde 1982 in Niedersachsen durchgeführt (Kenneweg und Liesebach 1984). Landesweite Inventuren in Baden-Württemberg, Niedersachsen, Saarland und regionale Inventuren in Bayern, Hessen, Nordrhein-Westfalen und Rheinland-Pfalz beruhten auf streifenweisen Luftbildbefliegungen im Maßstab 1:3000 - 1:6000. Der Vitalitätszustand von Einzelbäumen wurde in systematischen Stichproben erhoben und auf Bestandesebene zusammengefaßt. Zur visuellen Interpretation wurden einfache Auswertungsgeräte wie Spiegelstereoskope und Linien- und Punktrasterfolien eingesetzt.

Seit Ende der 80er Jahre fanden verstärkt analytische Stereoauswertungssysteme Eingang in die forstliche Praxis. Anwendungsbeispiele sind das Auffinden von Luftbildstichproben, die Abgrenzung von Schadensflächen und die Auswertung von Folgeaufnahmen zur Erfassung von Veränderungen (Akça 1989, Scherrer et al. 1994, Mauser 1998). Im Vordergrund standen bestandesbezogene Analysen im Rahmen regionaler Luftbilderhebungen (z. B. Ackermann und Hartmann 1992, Ackermann et al. 1996).

Mehrere Untersuchungen am Institut für Forsteinrichtung und Ertragskunde belegen die Eignung der fotogrammetrischen Erfassung von Einzelbäumen aus großmaßstäbigen CIR-Luftbildern. Kronendimensionen und Vitalitätszustand wurden in Zeitreihen großmaßstäbiger Luftbilder erfasst. Eine Vitalitätsverbesserung von Fichten und Eichen nach Düngungsmaßnahmen konnte nachgewiesen werden (Akça et al. 1990, 1991, 1995, 2000, Feldkötter et al. 1996). Mozgawa (1984) führte fotogrammetrische Kronen- 
messungen in Luftbildern durch und stellte Veränderungen der Kronenform von Fichten aufgrund eines verminderten Höhenwachstums fest.

Erste Konzepte zur automatischen Interpretation von Luftbildern wurden bereits Anfang der 70er Jahre entwickelt (z. B. Akça 1970, Tschupke 1974) und von Kim (1988) weitergeführt. Mikrodensitometrische Messungen in Schwarzweiß- und Farbinfrarot-Luftbildern dienten zur Analyse von Farb- und Texturmerkmalen. Zur Klassifikation wurden einfache Schwellenwertverfahren und lineare Diskriminanzanalysen eingesetzt, um in hierarchischen Verfahren Wald- und Nichtwald, Baumarten und Vitalitätsstufen zu unterscheiden. Mansberger (1992) setzte bereits digitalisierte CIRLuftbilder ein und entwickelte ein Verfahren zur digitalen Unterstützung der Interpretation der Vitalität von Waldbäumen. Er berechnete einen Differenzkanal zwischen den spektralen Bereichen des nahen Infrarot und Rot. Diese werden als einziges Merkmal zur statistischen Klassifikation der Kronenverlichtung eingesetzt. Beisch (1996) klassifizierte Fichtenkronen in Farbinfrarot-Luftbildern mit dem gleichen Differenzkanal. Er kam zu der Schlußfolgerung, dass die Verwendung des Differenzkanales besser geeignet ist für die Unterscheidung der Vergilbungsstufen als zur Einschätzung der Kronenverlichtung.

Gleichzeitig wurden Methoden der digitalen Bildverarbeitung für die Auswertung multispektraler Flugzeugscannerdaten (z. B. BENDIX MS, FLI (Rock et al. 1988) sowie Daedalus ATM (Herrmann 1989, Baumgarten 1990, Kenneweg et al. 1991, Leckie et al. 1992, Levesque und King 2003) und AIS-2 (Riggs und Running 1991)) weiterentwickelt. Im Vordergrund standen pixelbasierte Verfahren und der Einsatz von Bildverbesserungen zur Klassifikation von Baumarten und Vitalitätsstufen. Baumgarten (1990) verwendete Daedalus-Scannerdaten und setzte zur Klassifikation der Kronenverlichtung von Buchen und Eichen einen transformierten Normalisierten DifferenzVegetationsindex (NDVI) ein. Schramm et al. (1989) untersuchten Eichen, Buchen und Kiefern im Stadtwald Frankfurt mit multispektralen Daedalus Scannerdaten. Die Signaturanalysen ergaben geringe Unterschiede zwischen den Laubholzbaumarten, wodurch Fehlklassifikationen entstanden. Mit der ausschließlichen Verwendung der spektralen Signaturen eines Daedalus Scanners war eine Trennung der Eichen und Buchen nicht möglich. Scheer (2000) führte Signaturanalysen in CIR-Luftbildern an Fichten mit verschiedenen Kronenverlichtungsgraden durch. In einer Diskriminanzanalyse bestätigte er die Eignung der zweiten Hauptkomponente der Farbkanäle eines Orthofotos für die Vitalitätsbeurteilung. Yuan et al. (1991) setzten für die Klassifikation von Schäden in Ahorn-Beständen die spektralen Variablen NDVI, die ursprünglichen vier Kanäle und zusätzliche Texturmerkmale einer Video-Aufnahme ein. Koch et al. (2002) empfehlen die Verwendung von Texturmerkmalen zur digitalen Unterscheidung von Baumarten.

Halbritter (1996) untersuchte automatisch erstellte Oberflächenmodelle eines Waldbestandes in großmaßstäbigen Farbinfrarot-Luftbildern mit Hilfe eines digitalen fotogrammetrischen Systemes. Miller et al. (2000) erkannten neue Möglichkeiten der digitalen Oberflächenmodelle und Orthofotos zur Erfassung von Veränderungen in Waldbeständen mittels Zeitreihenanalysen. Hoffmann (2001) und Adler (2001) verwendeten digitale fotogrammetrische Systeme und digitaliserte CIR-Luftbilder zur Zustandsbeschreibung von Naturwaldreservaten bzw. Dauerbeobachtungsflächen. 
Zur Lösung des Problemes der unzureichenden Ergebnisse pixelbasierter Klassifikationen wird von Haenel et al. (1988) eine Objektentdeckung in Luftbildern vorgeschlagen. Sie unterscheiden zwei Methoden der Objekterkennung: die Bildsegmentierung, die z. B. zur Abgrenzung von Waldflächen eingesetzt werden kann und die direkte Objektsuche, wobei allgemeine Vorstellungen über ein Kronenmodell (z. B. zum Kronenrand der Krone abfallende Grauwerte oder annähernde Kreisgestalt) genutzt werden.

Die automatische Erkennung von Einzelbäumen aus digitalen Bildern eröffnet neue Möglichkeiten der Informationsgewinnung in Waldbeständen (Dralle 1997, Brandtberg 1999). Verschiedene Fallstudien wurden auf dem internationalen Forum „Automated Interpretation of High Spatial Resolution Digital Imagery for Forestry“ vorgestellt (Hill und Leckie 1998). Eine Zusammenstellung vielfältiger Lösungsansätze Ansätze und Algorithmen zur Erkennung von Einzelbäumen gibt Culvenor (2003). Beisch (1998) und Haala und Brenner (1999) kombinierten multispektrale Orthofotos mit geometrischen Informationen digitaler Oberflächenmodelle zur Klassifikation von Bäumen in Stadtgebieten.

\subsection{Waldwachstumskundliche Auswertungen und Luftbildanwendungen}

Der Zusammenhang zwischen Holzzuwachs und Kronenvariablen wurde bisher vorwiegend mit terrestrischen Methoden untersucht. In den älteren Arbeiten von Burger (1939, 1940, 1947) werden Zusammenhänge zwischen der Zuwachsleistung, Laubmasse und Blattoberfläche der Baumarten Fichte, Buche und Eiche beschrieben. Mayer (1958) untersuchte die Beziehungen zwischen Kronenschirm-, Kronenmantelfläche und dem Grundflächen- und Durchmesserzuwachs von Eichen. Spiecker (1991) stellte straffe Zusammenhänge zwischen Kronenbreite und Brusthöhendurchmesser (BHD) von Eichen fest und leitete daraus Orientierungshilfen für die Eingriffsstärke von Durchforstungen ab. Ebert und Rieger (2000) untersuchten den Zusammenhang zwischen terrestrisch erhobenen Kronendimensionen und dem BHD, Grundflächen- und Volumenzuwachs am Beispiel 60jähriger Eichen. Sie berichten über enge lineare Zusammenhänge zwischen Kronenmantelfläche, Kronenlänge und Kronenbreite mit dem BHD, Grundflächen- und Volumenzuwachs.

Die straffe Korrelation der im Luftbild ermittelten Kronenbreite und Brusthöhendurchmesser ist z. B. von Eule (1959) für Buche und von Kahleys und Klier (1968) für Fichte mittels einfacher Auswertungsgeräte untersucht worden. Akça $(1979,1984)$ erfasste die Kronendimensionen von Fichten eines Durchforstungsversuches mit Hilfe analoger Stereoauswertungsgeräte und großmaßstäbiger Luftbilder. Es wurden straffe Beziehungen zwischen Kronenbreite und BHD sowie zwischen Kronendimensionen und Grundflächenzuwachs festgestellt. Spellmann (1984) und Wolff (1992) nutzten fotogrammetrische Einzelbaummerkmale auf Basis von Luftbildstichproben zur Regressionsschätzung des Holzvorrates im Rahmen von Betriebsinventuren.

Die Möglichkeiten der Erfassung von Einzelbäumen mit analytischen Stereoauswer- 
tungsgeräten in großmaßstäbigen Luftbildern wurden von Akça (1989), Oester (1991) und Maxin (1991) untersucht und von Münch (1995) für die Erfassung von Bestandeslücken auf Naturwaldflächen eingesetzt. Aus einer Zeitreihenanalyse entwickelte Münch Konkurrenzindizes unter Verwendung der Baumhöhe und der Höhenänderung von Nachbarbäumen.

Herwitz et al. (2000) bestimmten Änderungsraten der Kronenschirmfläche von Eichen aus einer Reihe von Farb- und Farbinfrarot-Luftbildern der Maßstäbe 1:1500 bis 1:10000 über eine Zeitraum von 21 Jahren. 


\section{Material und Methoden}

\subsection{Untersuchungsflächen}

Die in der vorliegenden Arbeit untersuchten Dauerbeobachtungsflächen liegen in Rheinland-Pfalz in den Wuchsgebieten Westerwald, Hunsrück und Pfälzerwald und in Nordrhein-Westfalen im Wuchsgebiet Niederrheinische Tiefebene. Die Fichtenflächen 130, 131, 132 und 133 werden im deutschen Level 2-Programm mit Nr. 701, die Eichenflächen 405, WBO und WBK als Level 2-Fläche Nr. 705 bezeichnet. Die Teilflächen haben eine Größe von 0,1 bis 0,5 ha.

Die klimatischen und edaphischen Bedingungen der Eichenfläche 502 im Flachland unterscheiden sich von denen der anderen Flächen, die im Bergland liegen. Auf der Fläche 502 liegt eine Pseudovergleyung vor. Die mittleren Lufttemperaturen sind höher, die mittleren Niederschläge geringer als in den anderen Wuchsgebieten. Eine allgemeine Beschreibung der Waldbestände, Klima- und Standortsmerkmale der Untersuchungsflächen geben die Tabellen 3.1 und 3.2 wieder.

Tab. 3.1: Waldbestandesmerkmale der Untersuchungsflächen (aus BMELF 1997 und Schröck 1994)

\begin{tabular}{|l|c|c|c|c|c|}
\hline Hauptbaumart (HBA) & \multicolumn{2}{|c|}{ Fichte } & Buche & \multicolumn{2}{c|}{ Eiche } \\
\hline Flächen-Nr. & 108 & $130-133$ & 203 & 405 & 502 \\
Alter Hauptbaumart & $81(1989)$ & $67(1991)$ & $147(1989)$ & $169-196$ & 120 (1996) \\
& & & & $(1986)$ & \\
Grundflächenanteil & $100 \%$ & $100 \%$ & $100 \%$ & $100 \%$ & $80 \%$ \\
Nebenbaumart (NBA) & - & - & - & Buche & Buche \\
Alter Nebenbaumart & - & - & & $95(1986)$ & $66-110$ \\
& & & & & $(1996)$ \\
Grundflächenanteil & - & - & - & Unterstand & $20 \%$ \\
Jahr der Aufnahme & 1989 & 1995 & 1989 & 1995 & 1995 \\
Stammzahl n & $165[\mathrm{n}]$ & $461[\mathrm{n} / \mathrm{ha}]$ & $33[\mathrm{n}]$ & 204 [n/ha] & $216[\mathrm{n} / \mathrm{ha}]$ \\
Oberhöhe (h100) HBA & & $26,5 \mathrm{~m}$ & & $29,9 \mathrm{~m}$ & $27,7 \mathrm{~m}$ \\
Bestockungsgrad & 0,9 & & 0,8 & & \\
Bonität & $\mathrm{I} .9$ & & $\mathrm{I} .6$ & & \\
Vorrat [Vfm/ha] & & 492,1 & & 437,8 & 389 \\
Lfd. jährl. Zuwachs & & 14,0 & & 4,4 & $2,5-7,5$ \\
[Vfm/ha] & & & & & \\
\hline
\end{tabular}


Tab. 3.2: Klima- und Standortsmerkmale der Untersuchungsflächen (aus BMELF 1997 und Schröck 1994)

\begin{tabular}{|c|c|c|c|c|c|}
\hline Land & \multicolumn{4}{|c|}{ Rheinland-Pfalz } & \multirow{3}{*}{$\begin{array}{c}\begin{array}{c}\text { Nordrhein- } \\
\text { Westfalen }\end{array} \\
\text { Kleve } \\
\begin{array}{c}\text { Nieder- } \\
\text { rheinische } \\
\text { Tiefebene }\end{array} \\
\end{array}$} \\
\hline Forstamt & \multirow{2}{*}{\multicolumn{2}{|c|}{$\frac{\text { Montabaur }}{\text { Westerwald }}$}} & \multicolumn{2}{|c|}{ Merzalben } & \\
\hline Wuchsgebiet & & & Hunsrück & Pfälzer & \\
\hline Flächen-Nr. & 108 & 203 & $130-133$ & 405 & 502 \\
\hline Höhenlage & 470 & 365 & 634 & 547 & 30 \\
\hline $\begin{array}{l}\text { Niederschlag } \\
{[\mathrm{mm} / \mathrm{a}]}\end{array}$ & 850 & 850 & 1080 & 950 & $750-800$ \\
\hline $\begin{array}{l}\text { Lufttemperatur } \\
{\left[{ }^{\circ} \mathrm{C}\right]}\end{array}$ & 8 & 8 & 6 & 8 & $9,5-9,0$ \\
\hline Bodentyp & $\begin{array}{c}\text { mittelgr. } \\
\text { Braunerde }\end{array}$ & $\begin{array}{c}\text { mittelgr. } \\
\text { Braunerde }\end{array}$ & $\begin{array}{c}\text { tiefgr. } \\
\text { Braunerde }\end{array}$ & $\begin{array}{l}\text { sehr tiefgr. } \\
\text { Braunerde }\end{array}$ & $\begin{array}{l}\text { Pseudogley- } \\
\text { Braunerde }\end{array}$ \\
\hline Substrat & $\begin{array}{l}\text { Quarzit } \\
\text { (Unter- } \\
\text { devon) }\end{array}$ & $\begin{array}{c}\text { Ton- } \\
\text { schiefer }\end{array}$ & $\begin{array}{l}\text { Sandstein } \\
\text { mit } \\
\text { Decklehm }\end{array}$ & $\begin{array}{l}\text { Mittlerer } \\
\text { Buntsand- } \\
\text { stein }\end{array}$ & $\begin{array}{c}\text { kiesig- } \\
\text { sandige } \\
\text { Strauchmo- } \\
\text { räne }\end{array}$ \\
\hline Humusform & & & $\begin{array}{c}\text { feinhumus- } \\
\text { reicher } \\
\text { Moder }\end{array}$ & $\begin{array}{l}\text { Of-Mull, } \\
\text { z.T. mull- } \\
\text { artiger } \\
\text { Moder }\end{array}$ & $\begin{array}{c}\text { feinhumus- } \\
\text { armer } \\
\text { Moder }\end{array}$ \\
\hline
\end{tabular}

\subsection{Datengrundlage und Bildmaterial}

\subsubsection{Luftbilder}

Die Aufnahme der 49 CIR-Luftbilder wurde mit einer Längsüberdeckung von 60 bis $80 \%$ durchgeführt. Zwei sich überdeckende Luftbilder einer Reihenaufnahme bilden ein Stereopaar. Objektpunkte, die in dem Überdeckungsbereich abgebildet werden, sind die Grundlage für ein Stereomodell.

Die Befliegungen wurden in 1- bis 7-jährigen Abständen wiederholt. Zeitreihen über eine Dauer von 6 bis zu 11 Jahren konnten dokumentiert werden. Die mittleren Bildmaßstäbe liegen zwischen 1:2700 und 1:4300 bei einer Flughöhe von 900 bis 1900 m (Tab. 3.3), so dass ein Luftbild eine Flächengröße zwischen 38 und 85 ha abbildet. Die Flüge wurden in nördlicher oder südlicher Richtung durchgeführt, wobei kalibrierte 
Reihenmesskammern mit Normalwinkelobjektiven verwendet wurden. Eine Ausnahme ist die Befliegung der Fichten-Flächen 130-133 im Jahr 1997, bei der ein Weitwinkelobjektiv eingesetzt wurde.

Tab. 3.3: Technische Daten des verwendeten Luftbildmaterials

\begin{tabular}{|c|c|c|c|c|c|c|c|}
\hline Bildnr. & $\begin{array}{l}\text { Aufnahme- } \\
\text { datum }\end{array}$ & $\begin{array}{l}\text { Uhr- } \\
\text { zeit }\end{array}$ & $\begin{array}{l}\text { Kammer- } \\
\text { konstante } \\
{[\mathrm{mm}]}\end{array}$ & $\begin{array}{l}\text { Flug- } \\
\text { höhe } \\
{[\mathrm{m}]}\end{array}$ & $\begin{array}{l}\text { Mittl. } \\
\text { Maßstab }\end{array}$ & $\begin{array}{l}\text { Scan } \\
\text { auflsg. } \\
{[\mu m]}\end{array}$ & Bem. \\
\hline \multicolumn{8}{|c|}{ Fläche 108 (Fichte) } \\
\hline $175 / 176$ & 22.8 .1984 & 13.45 & 304,96 & 1220 & $\approx 1: 4000$ & - & Schatten \\
\hline $12 / 13$ & 25.9.1986 & & 305,03 & 1037 & $\approx 1: 3400$ & - & Schatten \\
\hline $17 / 18$ & 30.7 .1992 & & 304,96 & 1220 & $\approx 1: 4000$ & - & Rotstich \\
\hline $519 / 520$ & 3.8 .1995 & & 304,775 & 1219 & $\approx 1: 4000$ & - & Rotstich \\
\hline \multicolumn{8}{|c|}{ Fläche 203 (Buche) } \\
\hline $178 / 179$ & 22.8 .1984 & & 304,96 & 1311 & $\approx 1: 4300$ & - & Schatten \\
\hline $158 / 159$ & 21.8.1987 & 15.00 & 304,96 & 1220 & $\approx 1: 4000$ & - & Schatten \\
\hline $14 / 15$ & 30.7.1992 & & 304,96 & 1250 & $\approx 1: 4100$ & - & Rotstich \\
\hline $513 / 532$ & 3.8.1995 & & 304,775 & 1280 & $\approx 1: 4200$ & - & Rotstich \\
\hline \multicolumn{8}{|c|}{ Fläche 405 (Eiche) } \\
\hline $72 / 73$ & 20.7.1992 & 13.30 & 304,968 & 1790 & $\approx 1: 4100$ & 24 & \\
\hline $23 / 24$ & 17.7.1999 & 15.30 & 305,048 & 1700 & $\approx 1: 3800$ & 24 & \\
\hline \multicolumn{8}{|c|}{ Fläche 130-133 (Fichte) } \\
\hline 92/93/94 & 29.7.1992 & 12.47 & 304,968 & 1875 & $\approx 1: 4080$ & 24 & Rotstich \\
\hline $1038 / 1039$ & 22.9.1997 & 12.51 & 153,802 & 1280 & $\approx 1: 4230$ & 24 & Schatten \\
\hline \multicolumn{8}{|c|}{ Fläche 502 (Eiche) } \\
\hline $56 / 57 / 58$ & 8.9.1988 & 13.32 & 305,024 & 979 & $\approx 1: 3100$ & 24 & \\
\hline $152 / 153$ & 21.8.1989 & 14.09 & 305,024 & 986 & $\approx 1: 3100$ & 24 & \\
\hline $51 / 52$ & 30.7 .1990 & 15.15 & 305,024 & 950 & $\approx 1: 3000$ & 24 & \\
\hline $329 / 330$ & 29.7.1991 & 12.59 & 305,072 & 978 & $\approx 1: 3100$ & 24 & \\
\hline $19 / 20$ & 29.7.1992 & 11.19 & 305,072 & 916 & $\approx 1: 2900$ & 24 & \\
\hline 294/295/296 & 18.8.1993 & 13.37 & 304,811 & 896 & $\approx 1: 2800$ & 24 & \\
\hline $54 / 55$ & 3.8 .1994 & 13.23 & 304,811 & 1118 & $\approx 1: 3600$ & 24 & Rotstich \\
\hline $200 / 201$ & 24.7.1995 & 15.44 & 304,811 & 1138 & $\approx 1: 3700$ & 24 & Rotstich \\
\hline $295 / 296$ & 8.8.1997 & 12.26 & 305,024 & 1121 & $\approx 1: 3600$ & 24 & Gelb \\
\hline $5 / 6$ & 27.7.1998 & 10.31 & 305,327 & 1101 & $\approx 1: 3500$ & 24 & \\
\hline $245 / 246$ & 28.7.1999 & 14.57 & 305,573 & 1125 & $\approx 1: 3600$ & $12-96$ & \\
\hline
\end{tabular}


Der CIR-Film Kodak Aerochrome IR 2443 ist ein Umkehrfilm, der aus drei Schichten besteht, die für den grünen, roten und nahinfraroten Spektralbereich mit Wellenlängen von 0,4 bis $0,9 \mu \mathrm{m}$ empfindlich sind. Alle drei Schichten sind auch für den blauen Teil des Lichtspektrums empfindlich. Bereits während der Bildaufnahme wird der negative Einfluss des blauen Lichtes durch die Verwendung eines Objektiv-Gelbfilters eliminiert. Nach der Belichtung des CIR-Filmes mit grüner, roter und nahinfraroter Strahlung entstehen während der Filmentwicklung im belichteten Diapositiv die Grundfarben Gelb (yellow), Purpur (magenta) und Blaugrün (cyan). Durch subtraktive Farbmischung entsteht eine „Falschfarben“-Darstellung, da die nahinfrarote Strahlung für den Menschen nicht sichtbar ist. Beim CIR-Luftbildfilm wird dem nahinfraroten Licht die Farbe Rot, dem roten Licht die Farbe Grün und dem grünen Licht die Farbe Blau zugeordnet (Lillesand und Kiefer 1994). Hieraus resultiert die typische Farbdifferenzierung: Laubbaumkronen werden in kräftigen Rottönen abgebildet, Nadelbaumkronen in den dunkleren Farbtönen Purpur bis Violett. Abgestorbene Bäume mit einer geringen Reflexion im nahen Infrarot nehmen grünblaue Farben an.

Wie aus Abbildung 3.1 (a) hervorgeht, überlappen sich die spektralen Empfindlichkeitskurven des Kodak Aerochrome IR 2443, so dass die breiten Bänder der Spektralbereiche Blau-Grün, Grün-Rot und Rot-Nahinfrarot nicht deutlich abgegrenzt sind. Die Maxima der Sensitivität der Emulsionen liegen bei Wellenlängen von 0,55 $\mu \mathrm{m}, 0,65 \mu \mathrm{m}$ und $0,73 \mu \mathrm{m}$.

Die theoretischen Schwärzungskurven (Abb. 3.1 (b)) können in der Realität stark variieren in Abhängigkeit der produzierten Emulsionen, dem Alter des Filmes, den Lagerungsbedingungen und der Filmentwicklung. Die Folge ist eine stark schwankende Farbwiedergabe, die den Vergleich von Luftbildern verschiedener Aufnahmezeitpunkte erschwert (Voretzsch et al. 1986, WGRSA 2000). Eine Anpassung der Interpretationsschlüssel hinsichtlich der Farbmerkmale und die Durchführung terrestrischer Geländeerhebungen sind deshalb notwendige Arbeitsschritte zur Auswertung von Veränderungen aus Luftbildzeitreihen.

Die fotografische Qualität der Bilder in den Jahren 1992, 1994 und 1995 wird durch einen starken Rotstich vermindert. Die Kontrasteigenschaften des Kodak Aerochrome 2443 sind geringer im Vergleich mit panchromatischen Farbfilmen. Der Film bildet jedoch Objekte mit einer Auflösung von 32 - 63 Linienpaaren/mm (Eastman Kodak Company 2000) ab. Bei einer Flughöhe von 1000 m und Verwendung eines Normalwinkelobjektives können noch kontrastarme Details von etwa $5 \mathrm{~cm}$ Größe auf dem Luftbildfilm erkannt werden. Kenngrößen der verwendeten Filmcharge können durch Messungen der optischen Filmdichte von aufgenommenen standardisierten Farbkeilen bestimmt werden (Voretzsch et al. 1986). Eine andere Möglichkeit ist das Auslegen von Bodenreferenzplatten vor der Befliegung, deren Reflexionsvermögen bekannt ist (Fischer und von Kienlin 1987). Diese zusätzlichen Messungen wurden beim vorliegenden Luftbildmaterial nicht durchgeführt. 


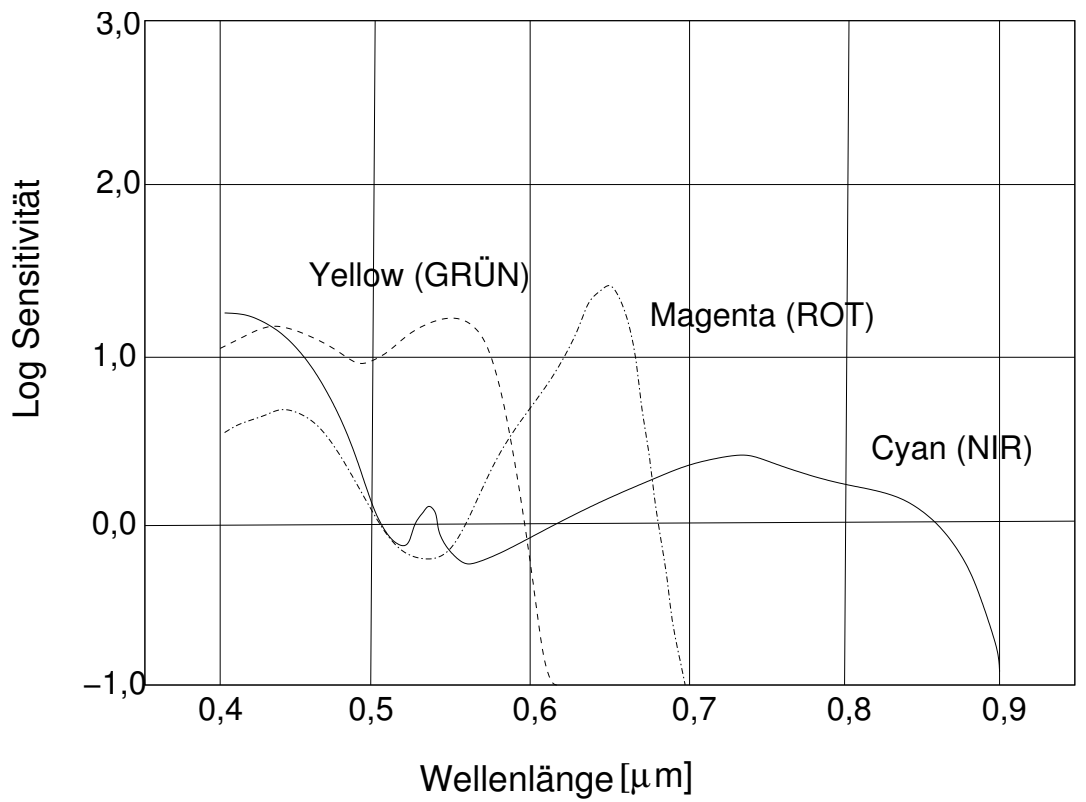

(a)

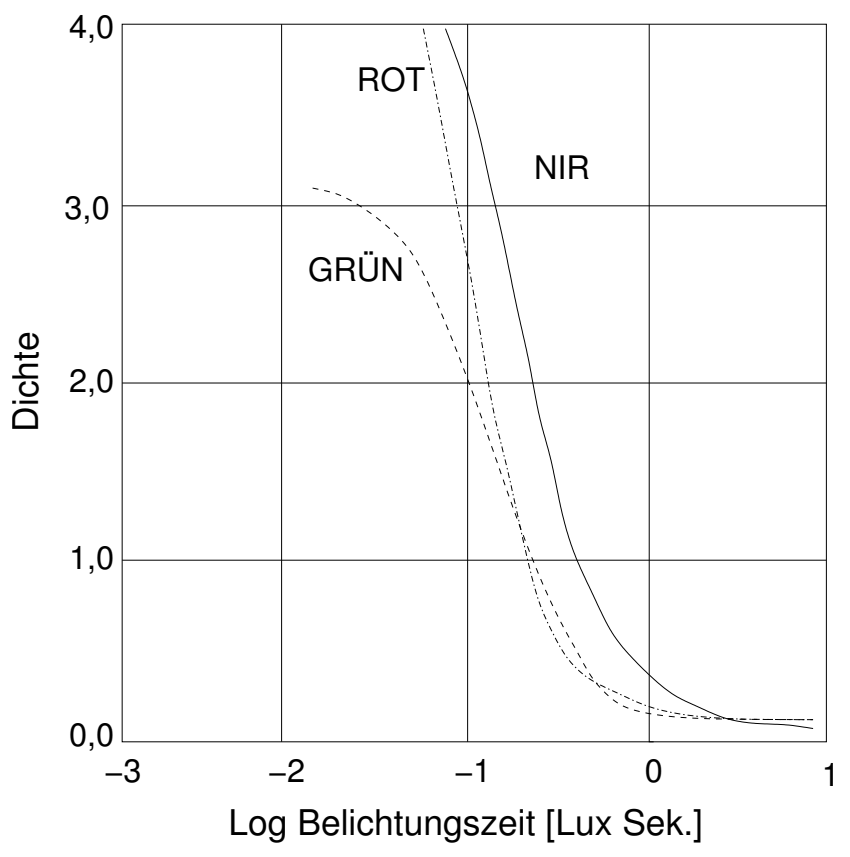

(b)

Abb. 3.1: (a) Spektrale Sensitivität und (b) theoretische Schwärzungskurven der Emulsionen des Farbinfrarot-Filmes Kodak Aerochrome II 2443 für die spektralen Bereiche Rot, Grün und nahes Infrarot (NIR) (nach Eastman Kodak Company 2000). 


\subsubsection{Terrestrische Erhebungen}

\subsubsection{Kronenverlichtung und -vergilbung}

Die Schätzung der Kronenverlichtung und -vergilbung wurde jährlich an Probebäumen auf den Untersuchungsflächen durchgeführt. Die Handbücher der Europäischen Kommission (Anonymus 2002) beschreiben die Methode der visuellen Erhebung des Kronenzustandes im Gelände. Das Kriterium der Blatt- bzw. Nadelvergilbung wird geschätzt, indem der Anteil vergilbter Blätter und Nadeln in $5 \%$-Stufen bewertet wird (Tab. 3.4).

Die Beurteilung der Kronenverlichtung wird im Vergleich zu einem voll belaubten Referenzbaum am gleichen Wuchsort in $5 \%$-Stufen durchgeführt. Dabei werden nur die Bereiche der Baumkrone berücksichtigt, die sich ohne störende Einflüsse durch benachbarte Baumkronen oder Lichtmangel entwickeln konnten. Kronenteile, die durch mechanische Beschädigung oder Beschattung beeinträchtigt sind, werden nicht in die Beurteilung einbezogen. Der Boniturbereich, d. h. der Kronenbereich, der zur Kronenzustandsbeurteilung dient, berücksichtigt im wesentlichen die Lichtkrone (ADW 1997).

Die Felderhebungen des Kronenzustandes werden jährlich in den Monaten Juli und August durchgeführt. Zusätzliche Informationen über die Einschätzung der Kronenverlichtung und des Insektenfraßes im Frühjahr standen auf der Eichenfläche 502 zur Verfügung.

Die Ergebnisse der Kronenzustandserhebungen werden zu einer Kombinationsstufe, auch Schadstufe genannt, zusammengefasst und dargestellt. Diese Klasseneinteilung und Zusammenführung der Merkmale Verlichtung und Vergilbung sind seit 1986 europäischer Standard (ADW 1997, s. Tab. 3.4). Mit der Verlichtungsstufe 4 (abgestorben) wird das stehende Totholz erfaßt.

Tab. 3.4: Herleitung der Kombinationsstufe aus Verlichtungsstufe (VL) und Vergilbungsstufe (VG)

\begin{tabular}{cc|ccccc}
\hline \multicolumn{6}{c|}{ Kronenverlichtung } & \multicolumn{5}{|c}{ Vergilbung } \\
VL- & VL- & $\mathbf{0}$ & $\mathbf{1}$ & $\mathbf{2}$ & $\mathbf{3}$ & VG-Stufe \\
Stufe & Prozent & $0-10 \%$ & $11-25 \%$ & $26-60 \%$ & $61-100 \%$ & VG-Prozent \\
\hline $\mathbf{0}$ & $0-10 \%$ & 0 & 0 & 1 & 2 & \\
$\mathbf{1}$ & $11-25 \%$ & 1 & 1 & 2 & 2 & Kombinations- \\
$\mathbf{2}$ & $26-60 \%$ & 2 & 2 & 3 & 3 & stufe \\
$\mathbf{3}$ & $61-99 \%$ & 3 & 3 & 3 & 3 & \\
\hline $\mathbf{4}$ & $100 \%$ & 4 abgestorben \\
\hline
\end{tabular}




\subsubsection{Dendrometrische Daten}

Der Durchmesser- und Grundflächenzuwachs von Einzelbäumen wurde für die entsprechenden Untersuchungszeiträume aus der Ablesung von Zuwachsmessbändern hergeleitet. Bei den Flächen 108 und 203 wurden diese aus zwei gemittelten BHD-Messungen bestimmt. Eine ertragskundliche Aufnahme mit Höhen- und Kronenansatzmessungen lag bei allen Versuchsflächen zu mindestens einem Zeitpunkt vor. Auf der Fläche 502 wurden außerdem in einer Vollaufnahme sämtliche Baumfußkoordinaten eingemessen.

Tab. 3.5: Terrestrisch erfasste Variablen auf den Untersuchungsflächen

\begin{tabular}{lccccc}
\hline Flächen-Nr. & 108 & $130-133$ & 203 & 405 & 502 \\
\hline Baumnr. & $\mathrm{x}$ & $\mathrm{x}$ & $\mathrm{x}$ & $\mathrm{x}$ & $\mathrm{x}$ \\
BHD & $\mathrm{x}$ & $\mathrm{x}$ & $\mathrm{x}$ & $\mathrm{x}$ & $\mathrm{x}$ \\
Zuwachsmessband & & $\mathrm{x}$ & & $\mathrm{x}$ & $\mathrm{x}$ \\
Baumhöhe & $\mathrm{x}$ & $\mathrm{x}$ & $\mathrm{x}$ & $\mathrm{x}$ & $\mathrm{x}$ \\
Kronenansatz & $\mathrm{x}$ & $\mathrm{x}$ & $\mathrm{x}$ & $\mathrm{x}$ & $\mathrm{x}$ \\
Baumfußkoordinaten & & & & & $\mathrm{x}$ \\
\hline Kronenverlichtung Sommer & $\mathrm{x}$ & $\mathrm{x}$ & $\mathrm{x}$ & $\mathrm{x}$ & $\mathrm{x}$ \\
Vergilbung & $\mathrm{x}$ & $\mathrm{x}$ & $\mathrm{x}$ & $\mathrm{x}$ & \\
Ursache des Ausscheidens & & $\mathrm{x}$ & & & \\
Kronenverlichtung Frühjahr & & $\mathrm{x}$ & & & $\mathrm{x}$ \\
Insektenfraß Frühjahr & & & & & $\mathrm{x}$ \\
\hline
\end{tabular}

\subsection{Untersuchungsmethoden}

\subsubsection{Relevante Eigenschaften von Luftbildaufnahmen}

\subsubsection{Reflexion von Waldbäumen und Waldbeständen}

Bei fotografischen Luftbildaufnahmen besteht kein direkter Kontakt mit den untersuchten Objekten. Die Information wird durch elektromagnetische Strahlung übertragen. Die Globalstrahlung (Sonnen- und Himmelsstrahlung) wird von Vegetationsoberflächen zum Teil aufgenommen (absorbiert), zurückgestrahlt (reflektiert) und durchgelassen (transmittiert) (Hildebrandt 1996).

Die Intensität und spektrale Zusammensetzung von Reflexion, Absorption und Transmission ist von physikalischen, chemischen und anatomischen Eigenschaften der Pflanzen abhängig. Die Blätter eines Baumes haben im Allgemeinen von allen oberirdischen 
Baumteilen die größte Oberfläche. In Fernerkundungsaufnahmen von Wäldern aus gröBerer Entfernung ist deshalb das Reflexionsverhalten der Blätter von Bedeutung.

Das Reflexionsverhalten von Blattorganen wurde intensiv unter Laborbedingungen und im Nahbereich mit Spektrometern untersucht. Die Ergebnisse wurden in Form von Reflexionskurven dargestellt, die die Rückstrahlung in Prozent der Einstrahlung einzelner Wellenlängenbereiche beschreiben. Im Folgenden werden einige Untersuchungsergebnisse für die Spektralbereiche des sichtbaren Lichtes $(0,4-0,7 \mu \mathrm{m})$ und des nahen Infrarot $(0,7-0,9 \mu \mathrm{m})$ dargestellt, da sie die Grundlage für die Erfassung von Waldbäumen und -beständen auf Farbinfrot-Luftbildern bilden.

Die spektrale Zusammensetzung der Reflexion von Blattorganen im sichtbaren Bereich von 0,4 bis $0,7 \mu \mathrm{m}$ wird im Wesentlichen durch die Lichtabsorption der Pigmente der obersten Blattschicht bestimmt. Grüne Chlorophylle und gelbe Carotinoide geben den Blättern ihre typische Farbe. Bei intakten Blattorganen wird das rote und blaue Licht stärker absorbiert als das grüne Licht. Nimmt der Chlorophyllgehalt durch Stresseinwirkung oder vor dem herbstlichen Blattfall der Laubbäume ab, wird eine höhere Reflexion und geringere Absorption mit einer Verschiebung des Reflexionsmaximums in Richtung des roten Spektralbereiches beobachtet (Buschmann und Nagel 1992, Hoffmann und Kirchhof 1989, Koch 1987).

Liegen Reflexionsmessungen in einer hohen spektralen Auflösung vor, ist ein weiteres Merkmal auffällig: Die Position des Wendepunktes im Anstieg des Reflexionssignales zum nahen Infrarot (,red edge“) verschiebt sich bei geringeren Chlorophyllgehalten hin zu kürzeren Wellenlängen (,blue shift“) (Rock et al. 1988, Westman und Price 1988). Dieses scheint ein für Pflanzen allgemein gültiges Schema der Reflexionsveränderung bei Stresseinwirkung zu sein (Abb. 3.2). Essery und Morse (1992) beobachten einen ähnlichen Verlauf der Reflexionskurven von jungen Fichten nach Experimenten zur Einwirkung von saurem Niederschlag und Ozon.

Die typischen phänologischen Veränderungen der Reflexion von Laubbäumen im Jahresverlauf - mit einem hohen Reflexionswert im sichtbaren Spektralbereich während des Laubaustriebes im Frühjahr, einem Absinken der Reflexionswerte im Sommer und einem Anstieg im Spätsommer bis zum herbstlichen Blattfall - sind vor allem auf die Änderung der Blattpigmentgehalte zurückzuführen, wie Koch (1987) am Beispiel der Rotbuche nachweist. Laubholzarten haben im Allgemeinen eine höhere Reflexion als Nadelholz (Fichte) (Hoffmann und Kirchhof 1989).

(Hoffmann und Kirchhof 1989) berichten über Laborversuche zum Einfluss der Blattstapelung. Bei Überlagerung von mehreren belaubten Ästen bei der Buche wurde keine Veränderung der Rückstrahlung im sichtbaren Spektralbereich beobachtet. Eine Trennung des Reflexionsverhaltens von Schatten- und Sonnenblättern der Buche konnte von Hoffmann und Kirchhof (1989) nicht durchgeführt werden. Koch (1987) beschreibt geringe Unterschiede bei Buchen und Fichten mit verschiedenen Blattverlustgraden im sichtbaren Spektralbereich.

Der zelluläre Aufbau eines Blattes ist von großer Bedeutung für das Reflexionsverhalten der Blattorgane bei Waldbäumen im nahen Infrarot $(0,7-0,9 \mu \mathrm{m})$ (Gausman 1977). Die Blattgewebestruktur wirkt sich besonders im nahen Infrarot aus, da in diesem Bereich wenig Strahlung absorbiert wird (Abb. 3.3). Ein geringeres Zellvolumen 


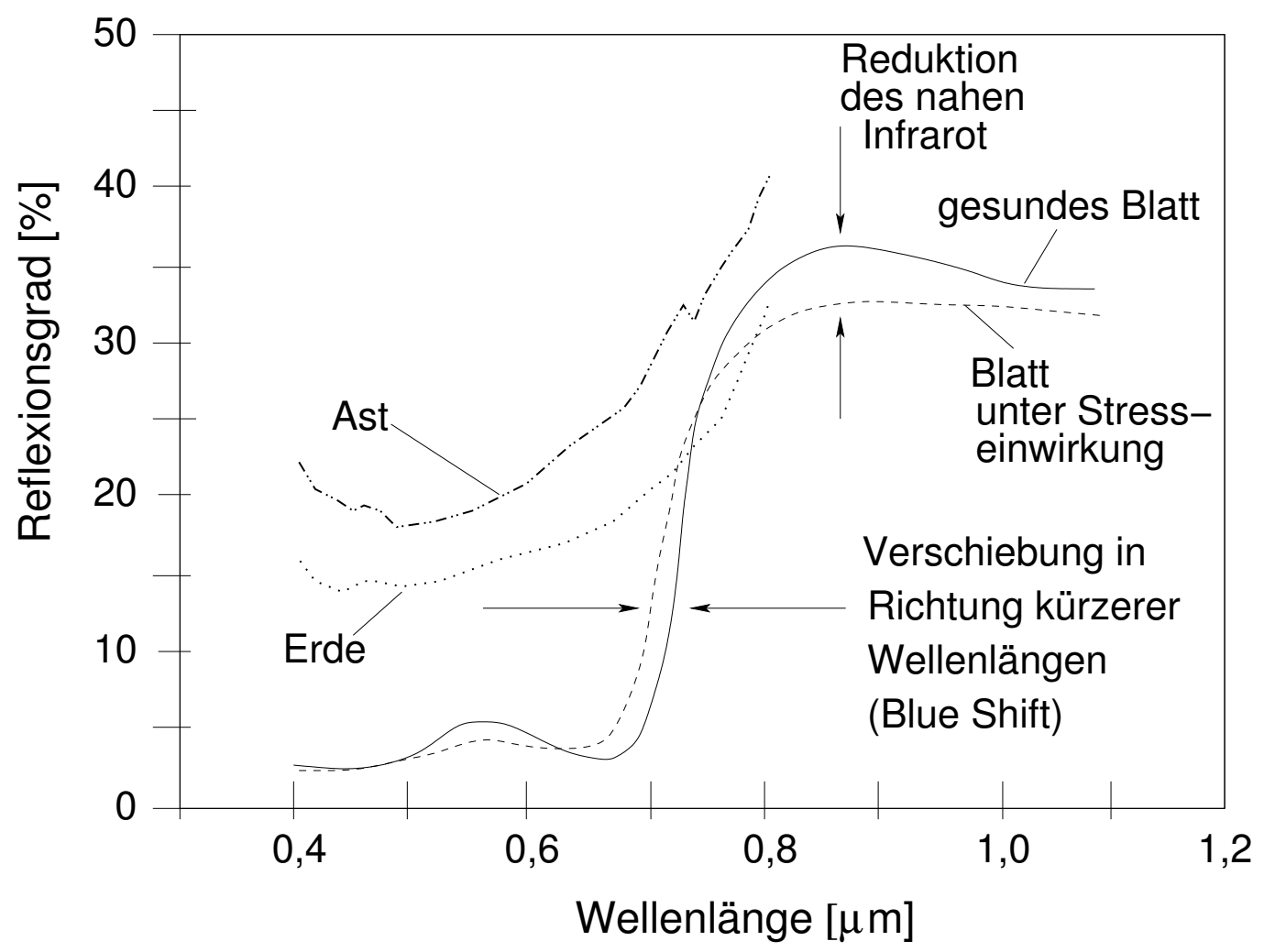

Abb. 3.2: Typische Reflexionskurven grüner Blätter im gesunden Zustand und unter Stresseinwirkung, Äste und unbedecktem Boden (verändert nach Reid 1992 und Buschmann und Nagel 1992)

(Westman und Price 1988) und die Verminderung der Dichte der Interzellularen (luftgefüllte Zellzwischenräume) verringert die Reflexion im nahen Infrarot durch Änderung der Lichtbrechung und -streuung innerhalb des Blattes (Buschmann und Nagel 1992). Eine Verminderung der Interzellulardichte tritt bei Geweberissen, Nekrosen oder infolge von Austrocknung bzw. Wassermangel auf. Nach Koch (1987) sowie Riggs und Running (1991) muss ein Wasserverlust allerdings sehr groß sein, um den Reflexionsgrad zu verringern. Der spektrale Bereich des mittleren Infrarots $(1,5-1,75 \mu \mathrm{m})$ ist zum Nachweis von Wassermangel bei Pflanzen besser geeignet (Tucker 1980), liegt aber außerhalb der Sensitivität des CIR-Filmes Kodak 2443.

Eine hohe Reflexion im nahen Infrarot weist im Allgemeinen auf eine gesunde Vegetation hin. Es gibt jedoch auch Ausnahmen von diesen Gesetzmäßigkeiten. Essery und Morse (1992) berichten von einer Erhöhung des nahen Infrarot bei jungen Fichten, die einer zweijährigen Behandlung mit Ozon und saurem Nebel unterlagen. Die Messungen wurden mit einem Feldspektrometer im Nahbereich und unter Vermeidung der Aufnahme der Bodenreflexion vorgenommen.

Eine Verminderung des Chlorophyllgehaltes bei vergilbten Blättern hat dagegen keine Bedeutung oder führt nur zu einer geringen Abschwächung der Reflexion im nahen 


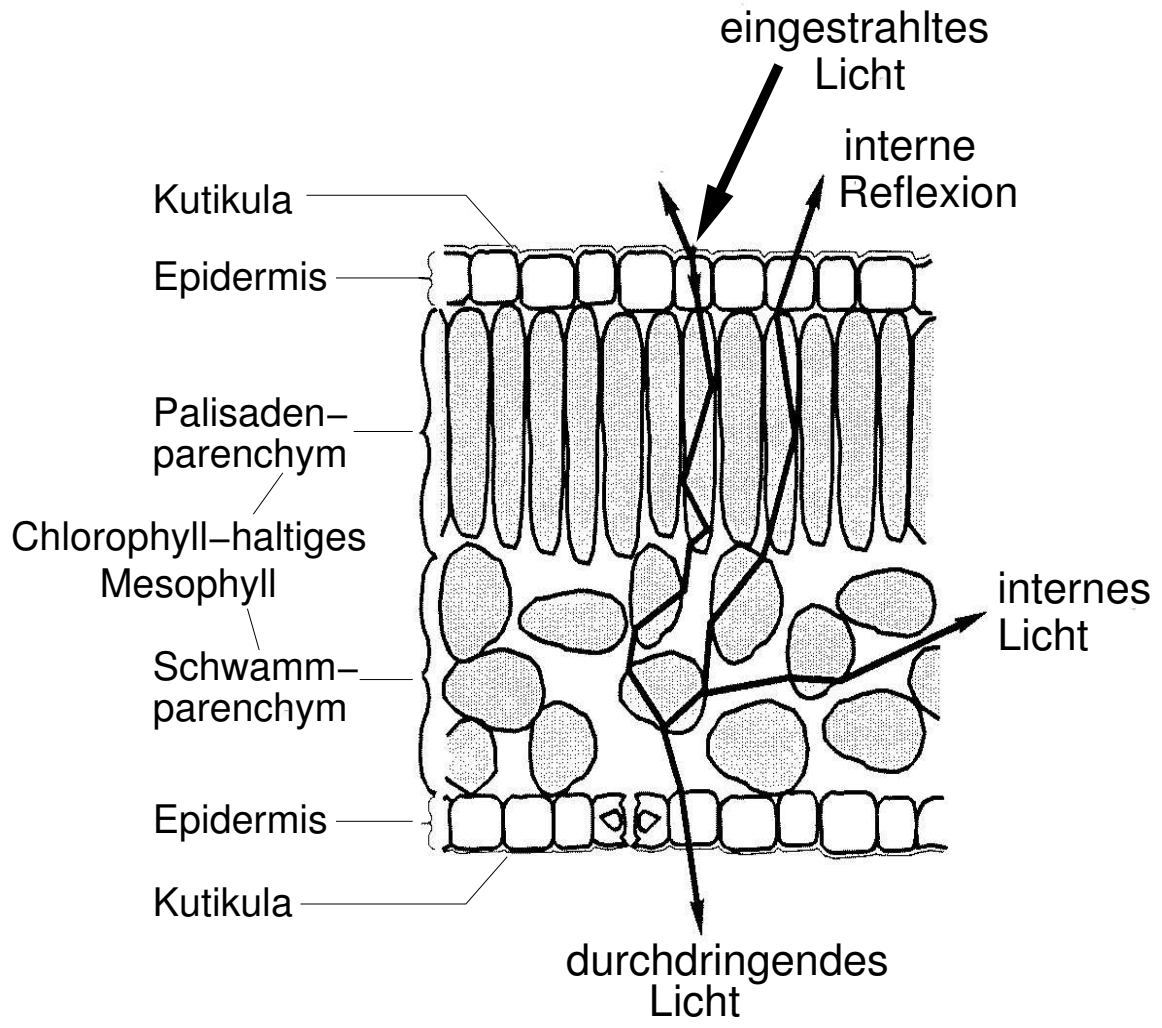

Abb. 3.3: Schematische Darstellung des Strahlungsweges durch ein Laubblatt (aus Buschmann und Nagel 1992)

Infrarot (Koch 1987).

Die Stapelung mehrerer Blätter bei der Buche ergibt eine deutliche Zunahme der Reflexion (hohe Transmission und Mehrfachreflexion) (Hoffmann und Kirchhof 1989), während zunehmende Blatt- oder Nadelverluste zu einer Abnahme der Reflexion im spektralen Bereich im Fall von Buchen und Kiefern führen (Landauer und Voß 1989, Koch 1987).

Die Unterscheidung von Laub- und Nadelholzarten wird im nahen Infrarot durch erhebliche Unterschiede der Höhe der nahinfraroten Rückstrahlung erleichtert. Diese sind auf anatomische Unterschiede im Nadel- / Blattaufbau zurückzuführen.

Reflexionsignale aus Fernerkundungsaufzeichnungen werden aus größerer Entfernung aufgenommen und erfassen nicht nur ein einzelnes Blatt, sondern integrieren den Einfluss von Ästen, Stammteilen oder Früchten. Die Reflexion von Ästen steigt allmählich vom blauen Spektralbereich zum nahen Infrarot an. In Waldbeständen mit geringem Überschirmungsgrad, in denen auch der Untergrund beleuchtet wird, trägt dieser zum Reflexionssignal der Baumkronen bei. Die Reflexion von braunem Boden unterscheidet sich wesentlich von grünen Blättern, da ein Anstieg und Wendepunkt zum nahen Infrarot fehlt und die Reflexion im nahen Infrarot geringer ausfällt (vgl. Abb. 3.2). 
Schatten innerhalb der Krone, die durch die artspezifische Architektur der Baumkronen entstehen oder bei stärkeren Verlusten der Blattorgane auftreten, können einen größeren Einfluss auf die Reflexionswerte bekommen als Blattorgane (Koch 1987). In lichten Beständen mit Bodenvegetation und hohen Nadel- / Blattverlusten kann es zu einer Überstrahlung und Erhöhung der Reflexion im nahen Infrarot kommen. Wird der Reflexionsgrad von Waldbeständen mit einer verringerten geometrischen Auflösung aufgenommen, werden die Schattenanteile zwischen den Baumkronen erfasst, was zu einer verminderten Reflexion im nahen Infrarot führt.

\subsubsection{Atmosphärische Einflüsse}

Die Zusammensetzung der reflektierten Strahlung wird beim Durchdringen der Atmosphäre verändert, was kontrastarme Farbaufnahmen zur Folge haben kann. Eine Ursache liegt im Aerosolgehalt der Atmosphäre, der zu einer vermehrten Lichtstreuung führt (Schneider 1989). Zur Korrektur können komplexe Atmosphärenmodelle verwendet werden, wenn zeitgleich zur Bildaufnahme entsprechende atmosphärische Messungen (z. B. Luftdruck, Ozongehalt, relative Luftfeuchtigkeit, Aerosolgehalt) vorliegen. Radiometrische Korrekturen können außerdem aus empirisch gemessenen Reflexionsgraden hergeleitet werden. Hierzu werden Probeflächen und Objekte mit Feldspektrometern zum Zeitpunkt der Bildaufnahme aufgesucht und die Globalstrahlung und spezifische Reflexion der Referenzflächen gemessen. Dies ist mit einem erheblichen Aufwand verbunden.

Eine Korrektur der atmosphärischen Einflussfaktoren wurde bei den verwendeten Luftbildern nicht durchgeführt. Die Flughöhen von 900 - 1900 m sind vergleichsweise gering. In der vorliegenden Untersuchung wurde zunächst ein relativer Vergleich innerhalb eines Bildjahrganges angestrebt.

\subsubsection{Sonnen- und Beobachtungsgeometrie}

Aus Messungen in Luftbildern und multispektralen Scanneraufnahmen ist bekannt, dass der Bildort, die Sonnen- und die Sensorgeometrie die Ergebnisse digitaler Klassifikation und Interpretation beeinflussen (Lillesand und Kiefer 1994, Kleman 1987, 1986). Für die digitale Auswertung und die Herstellung der digitalen Orthofotos wurden deshalb Luftbilder ausgewählt, bei denen die Untersuchungsflächen in der Nähe des Bildhauptpunktes liegen.

Bei flächenabbildenden Sensoren aus dem Flugzeug mit geringen Flughöhen kann der Einfluss der Blickrichtung sehr ausgeprägt sein. Die Beziehung zwischen den Bildkoordinaten und der zugehörigen Blickrichtung kann durch den Zenitwinkel und das Azimut gegeben werden. In Tabelle 3.6 ist der Zenitwinkel der Beobachtungsrichtung näherungsweise aus den absoluten Orientierungsdaten (Projektionszentren, Flughöhe über Grund) in Bezug zum Mittelpunkt der Untersuchungsflächen berechnet worden. Der Zenitwinkel der Blickrichtung liegt nur im Befliegungsjahr 1989 über $5^{\circ}$, so dass alle Bilder eines 12jährigen Untersuchungszeitraumes hinsichlich dieses Einflussfaktors vergleichbar sind. Für die digitale Klassifikation wurde nur ein kleiner Bildbereich 
in der Nähe der Untersuchungsflächen ausgewertet. Für eine Auswertung der gesamten Fläche eines Luftbildes kann eine zusätzliche Bestimmung des Azimutes der Beobachtungsrichtung vorteilhaft sein. Der Sonnen- sowie der Blickrichtungsazimut bestimmen den Mitlicht- und Gegenlichtbereich eines Luftbildes. Holopainen und Wang (1998), Kraus (1990) und Mansberger (1992) stellen radiometrische Korrekturmethoden unter Berücksichtigung der Beobachtungsrichtung vor. Nach Transformation der Bildkoordinaten in ein Koordinatensystem, dessen y-Achse nach dem Sonnenazimut ausgerichtet ist, gibt die transformierte y-Koordinate an, ob sich ein Objekt im Mitlicht- oder Gegenlichtbereich des Luftbildes befindet (Abb. 3.4). Hieraus lassen sich objektspezifische Regressionen zur radiometrischen Korrektur ableiten (z. B. Holopainen und Wang 1998). Der Sonnenzenitwinkel zum Zeitpunkt der Bildaufnahmen der Fläche 502 liegt in allen Jahren, mit Ausnahme 1988, über 50.

Eine andere Möglichkeit der Verringerung des Einflusses der Sonnen- und Beobachtungsrichtung besteht in der Verwendung von Differenz- oder Ratiokanälen (Hildebrandt 1996, Kraus 1990).

Tab. 3.6: Sonnengeometrie (Sonnenzenitwinkel und -azimut) und Zenit der Beobachtungsrichtung (Blickwinkel) einer Luftbildzeitreihe (Fläche 502)

\begin{tabular}{cccccc}
\hline Bildnr. & Flächennr. & Jahr & Sonnenzenitwinkel & Sonnenazimut & Blickwinkel $\left[^{\circ}\right]$ \\
\hline 57 & 502 & 1988 & 43.7 & 184.2 & 3,0 \\
152 & 502 & 1989 & 50.1 & 188.2 & 8,4 \\
51 & 502 & 1990 & 52.1 & 217.6 & 4,1 \\
330 & 502 & 1991 & 56.0 & 161.7 & 6,8 \\
20 & 502 & 1992 & 51.7 & 126.3 & 2,3 \\
295 & 502 & 1993 & 51.7 & 179.2 & 2,9 \\
54 & 502 & 1994 & 55.6 & 172.2 & 4,5 \\
200 & 502 & 1995 & 50.3 & 228.4 & 3,0 \\
295 & 502 & 1997 & 51.4 & 150.4 & 2,8 \\
6 & 502 & 1998 & 51.7 & 112.9 & 3,7 \\
245 & 502 & 1999 & 51.7 & 211.3 & 0,4 \\
\hline
\end{tabular}

\subsubsection{Technische Grundlagen der Luftbildauswertung}

\subsubsection{Analytischer Stereoplotter}

Die Datenerfassung der visuellen fotogrammetrischen Auswertung auf den Untersuchungsflächen 203 und 108 sowie Kontrollmessungen im Vergleich zu den digitalen Fotogrammetrischen Systemen fanden am analytischen Stereoplotter KERN DSR 11/18 


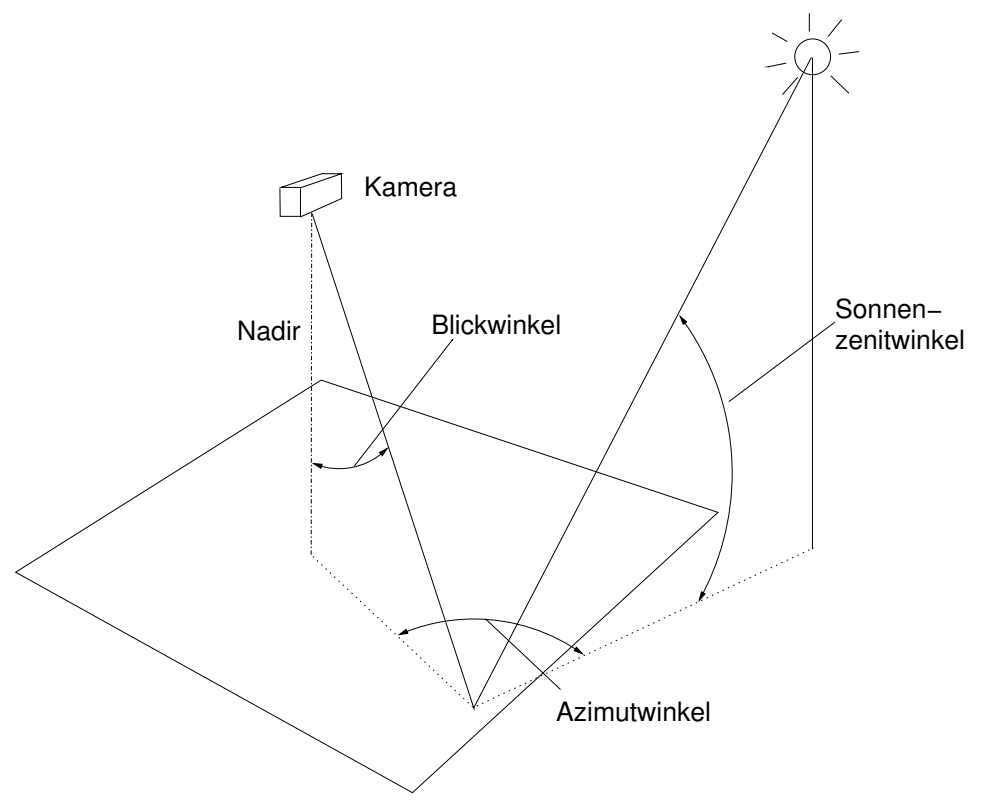

(a) Blickwinkel und Sonnenzenitwinkel

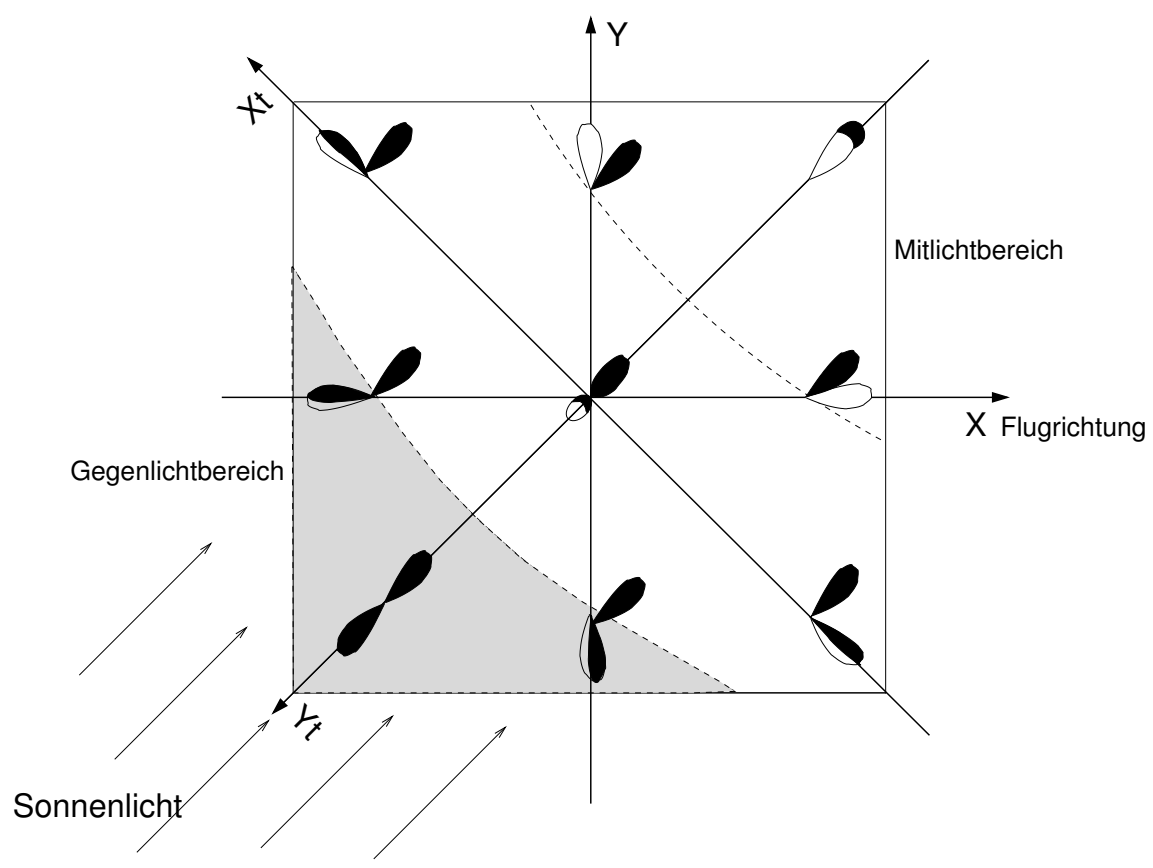

(b) Transformiertes Bildkoordinatensystem (Xt, Yt) zur Bestimmung des Mitlicht- und Gegenlichtbereiches

Abb. 3.4: Effekte der Sonnen- und Beobachtungsrichtung in Luftbildern (verändert nach Lillesand und Kiefer 1994 (a) und Mansberger 1992 (b)) 
statt, im folgenden kurz als DSR 18 bezeichnet. Analytische Stereoplotter dienen zur Berechnung der Positionen von Objektpunkten aus den korrespondierenden Bildkoordinaten von zwei sich überlappenden Stereofotografien. Es sind universelle dreidimensionale Kartiergeräte, die X, Y und Z in einem kartesischen Objektkoordinatensystem produzieren, wenn sie sorgfältig orientiert und kalibriert sind. Das DSR 18 besteht aus einem Hauptrechner, einem Steuerrechner, digitalen Encodern zur Messung der Position der Bildträgerplatten, Servomotoren, die auf Signale des Steuerrechners ansprechen und einem Stereobetrachtungsystem mit Bildträgerplatten. Die Komponenten werden schematisch in Abb. 3.5 dargestellt. Eine Besonderheit des DSR 18 sind große Bildträgerplatten, auf denen zwei Luftbildstereopaare (vier Luftbilder der Größe $23 \mathrm{x}$ $23 \mathrm{~cm}$ ) gleichzeitig orientiert werden. Bei Zeitreihenanalysen werden Luftbildmodelle verschiedener Aufnahmejahre mit einer geringen Verzögerung gewechselt und Veränderungen in einem direkten Vergleich festgestellt.

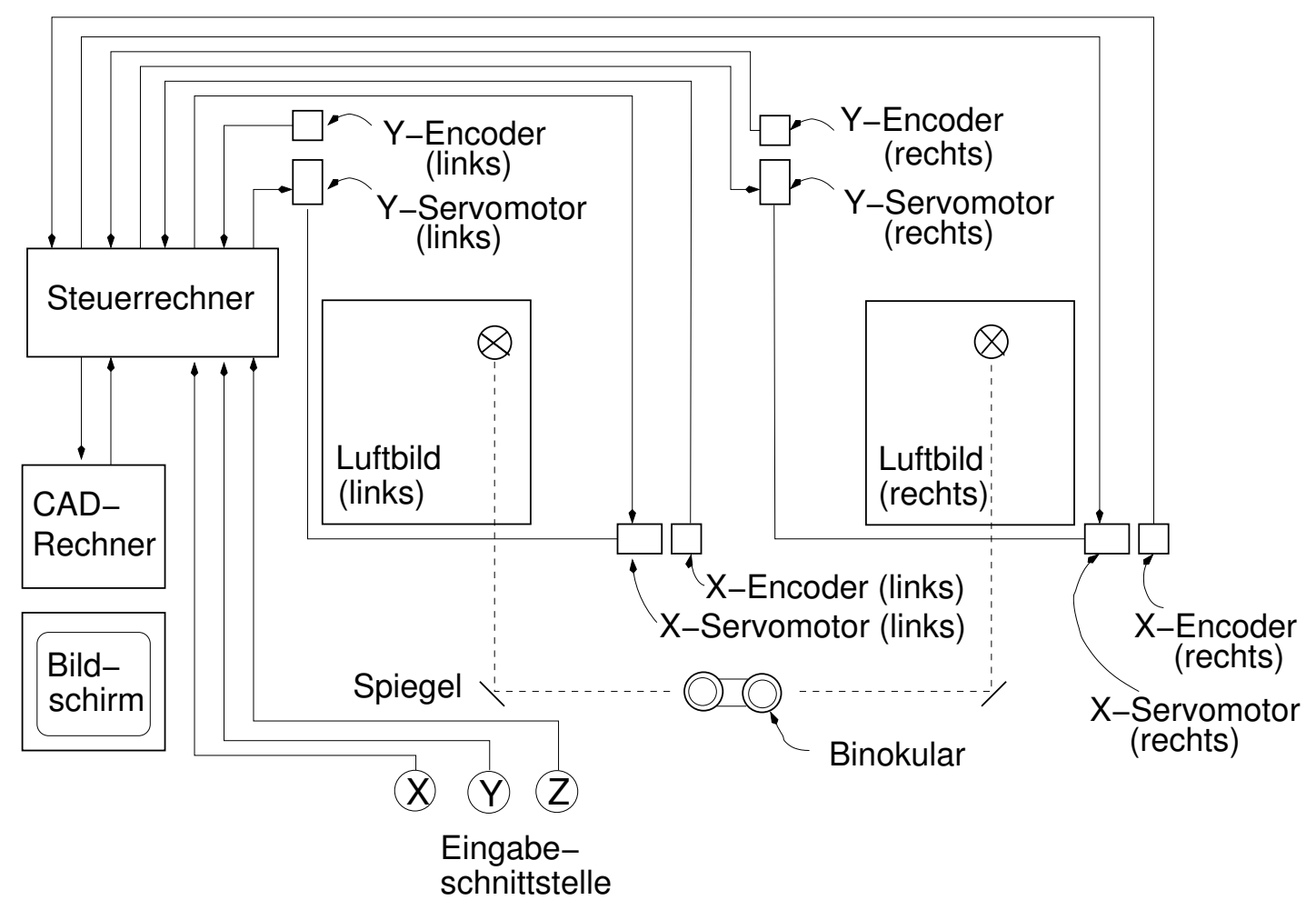

Abb. 3.5: Schematische Darstellung der Komponenten eines analytischen Stereoplotters (verändert nach Wolf und Dewitt 2000)

Die Grundfunktionen eines analytischen Stereoplotters sind genaue Messungen von $\mathrm{x}$ - und $\mathrm{y}$-Koordinaten in beiden Bildern eines Stereopaares und das Auffinden definierter x- und y-Bildkoordinaten. Der Hauptrechner übernimmt die Kommunikation mit dem Auswerter über verschiedene Programme zur Instrumentenkalibrierung, Bildorientierung und Datenerfassung (AVIOSOFT-Systemsoftware (Softmap 1999)). Der Steuerrechner ist über eine Schnittstelle mit dem Hauprechner verbunden. In einer Echt- 
zeitschleife werden Änderungen der verschiedenen Eingabemöglichkeiten des Auswerters akzeptiert. Der Steuerrechner ermittelt die neue Position der Bildträgerplatten, die über Servomotoren nachgeführt werden (Abb. 3.5). Die interne Messgenauigkeit ist sehr hoch und beträgt $3 \mu \mathrm{m}$. Nach abgeschlossener Bildorientierung können Messungen in einem dreidimensionalen Modell durchgeführt werden. Das Betrachtungssystem besteht aus einem Binokular mit Zoom-Einrichtung, mit dem die Diapositive in originaler Filmauflösung gesehen werden können. Messmarken werden in den Strahlengängen des Binokulars eingespiegelt. Die Position eines Punktes wird bestimmt, indem der Auswerter die Messmarke auf einen Modellpunkt aufsetzt.

\subsubsection{Bildorientierung}

Ein Stereomodell wird mathematisch nach drei Orientierungsschritten (innere, relative und absolute Orientierung) definiert (Kraus 1997, Hildebrandt 1996). Die Verfahrensschritte sind bei den analytischen und digitalen fotogrammetrischen Systemen im Wesentlichen gleich.

Zunächst werden zwei analoge Diapositive auf den Bildträgerplatten des Stereoplotters positioniert bzw. die digitalen Luftbilder im Orientierungsmodul des digitalen fotogrammetrischen Systems geladen. Dann werden x- und y-Koordinaten in den analogen bzw. digitalen Bildern gemessen. Diese Koordinaten werden in ein Bildkoordinatensystem transformiert, dessen Ursprung das Projektionszentrum darstellt. Die Gesetze der Zentralperspektive können mathematisch als Kollinearitätsbedingung formuliert werden. Sie besagt, dass das Projektionszentrum (eines Fotos), jeder Objektpunkt und dessen Abbildung im Foto auf einer Geraden in einem dreidimensionalen Koordinatensystem liegen. In zwei Kollinearitätsgleichungen (eine Gleichung für die x-Bildkoordinate und eine Gleichung für die y-Bildkoordinate) können die Beziehungen dargestellt werden (für eine ausführliche Herleitung s. Wolf und Dewitt (2000), Kraus (1997)). Die äußere Orientierung eines Luftbildes wird durch die Lage des Projektionszentrums im Raum und die Richtung der drei Drehwinkel Längsneigung $\varpi$, Querneigung $\phi$ und Kantung $\kappa$ beschrieben.

Die Beziehung zwischen Bild- und Objektkoordinaten (Landeskoordinaten) wird über Passpunkte hergestellt. Passpunkte sind Punkte, deren Landeskoordinaten bekannt sind und die auf den Luftbildern identifiziert werden könnnen. Für die Durchführung der absoluten Orientierung sind theoretisch mindestens zwei Lage- und drei Höhenpasspunkte zu bestimmen. In der Praxis ist eine Bestimmung mit mindestens vier Vollpasspunkten zu empfehlen, die möglichst weit auseinander und in der Nähe der Modellecken liegen sollten. Eine zusätzliche Überbestimmung dient der Kontrolle. Bei den durchgeführten Untersuchungen wurden die Passpunktkoordinaten aus vorliegendem Kartenmaterial (Deutsche Grundkarte oder Luftbildkarte im Maßstab 1: 5000) bestimmt. Für die Fläche 502 standen außerdem signalisierte Passpunkte zur Verfügung, die vor den Bildflügen tachymetrisch eingemessen wurden. Für größere Projekte kann eine Einsparung der Passpunktbeschaffung mit Hilfe einer Aerotriangulation sinnvoll sein. Im DFS SoftPlotter ist ein Programm zur Bündelblockausgleichung enthalten, das 
eine gleichzeitige Orientierung von mehr als zwei Einzelbildern in einem Schritt erlaubt (Jacobsen 2002, Kraus 1997).

Die relativen Lage- und Höhengenauigkeiten der absoluten Orientierung sind für die Beurteilung des digitalen fotogrammetrischen Systems „SoftPlotter“ gegenüber dem analytischen Stereoplotter DSR 18 von Bedeutung. Die geringsten Fehler in der Größenordnung unter 0,5 m wurden in 11 Modellen mit den signalisierten Passpunkten der Fläche 502 mit dem „SoftPlotter“ erreicht. Der Modellfehler der übrigen Luftbilder liegt in allen Fällen unter 3 m. Die Qualität der Passpunkte hat einen größeren Einfluss auf das Orientierungsergebnis als die Wahl eines digitalen oder analytischen Auswertungssystemes. Für eine Überprüfung der absoluten Genauigkeit sollte eine Überbestimmung der Passpunkte erfolgen, die für die Fläche 502 gegeben war. Im Fall der anderen Untersuchungsflächen war eine optimale Anzahl und Verteilung der Passpunkte in den Luftbildmodellen häufig nicht zu realisieren. Hier wurden zusätzliche Passpunkte fotogrammetrisch bestimmt und in andere Bildjahrgänge übertragen (Tab. 3.7). 
Tab. 3.7: Güte der absoluten Bildorientierung (RMS-Fehler, *=Restabweichung Lage, $* *=$ Restabweichung Höhe)

\begin{tabular}{|c|c|c|c|c|c|}
\hline Bildnr. & Jahr & Anzahl Paßpunkte & $\begin{array}{l}\text { Lage } \\
\mathrm{x}[\mathrm{m}]\end{array}$ & $\begin{array}{c}\text {-fehler } \\
\mathrm{y}[\mathrm{m}]\end{array}$ & $\begin{array}{c}\text { Höhenfehler } \\
\text { z [m] }\end{array}$ \\
\hline Fläche 108 & (Fichte) & DSR 18 & & & \\
\hline $175 / 176$ & 1984 & 7 & $\pm 0,42 *$ & & $\pm 0,16^{* *}$ \\
\hline $12 / 13$ & 1986 & 7 & $\pm 0,34^{*}$ & & $\pm 0,29 * *$ \\
\hline $17 / 18$ & 1992 & 7 & $\pm 0,24^{*}$ & & $\pm 0,33 * *$ \\
\hline $519 / 520$ & 1995 & 7 & $\pm 0,71^{*}$ & & $\pm 0,27 * *$ \\
\hline Fläche 203 & (Buche) & DSR 18 & & & \\
\hline $178 / 179$ & 1984 & 6 & $\pm 0,54^{*}$ & & $\pm 0,27 * *$ \\
\hline $158 / 159$ & 1987 & 6 & $\pm 1,16^{*}$ & & $\pm 0,52 * *$ \\
\hline $14 / 15$ & 1992 & 6 & $\pm 0,83^{*}$ & & $\pm 0,26^{* *}$ \\
\hline $531 / 532$ & 1995 & 6 & $\pm 0,69 *$ & & $\pm 0,18^{* *}$ \\
\hline Fläche 405 & (Eiche) & SoftPlotter & & & \\
\hline $72 / 73$ & 1992 & 7 & 0,31 & 0,42 & 0,48 \\
\hline $23 / 24$ & 1999 & 15 & 1,05 & 1,07 & 0,41 \\
\hline Fläche 130-133 & (Fichte) & SoftPlotter & & & \\
\hline 92/93/94 & 1992 & 12 & 1,93 & 2,29 & 2,56 \\
\hline $1038 / 1039$ & 1997 & 13 & 0,76 & 1,52 & 0,4 \\
\hline Fläche 502 & (Eiche) & SoftPlotter & & & \\
\hline $56 / 57 / 58$ & 1988 & 8 (7 signalisiert) & 0.18 & 0,19 & 0,28 \\
\hline $152 / 153$ & 1989 & 4 (2 signalisert) & 0,45 & 0,40 & 0,34 \\
\hline $52 / 52$ & 1990 & 8 (8 signalisiert) & 0,05 & 0,05 & 0,25 \\
\hline $329 / 330$ & 1991 & 7 (6 signalisiert) & 0,16 & 0,08 & 0,26 \\
\hline $19 / 20$ & 1992 & 6 (6 signalisert) & 0,07 & 0,04 & 0,11 \\
\hline $294 / 295 / 296$ & 1993 & 9 (8 signalisert) & 0,42 & 0,26 & 0,20 \\
\hline $54 / 55$ & 1994 & 9 (8 signalisert) & 0,40 & 0,29 & 0,20 \\
\hline $200 / 201$ & 1995 & 8 (8 signalisiert) & 0,45 & 0,26 & 0,44 \\
\hline $295 / 296$ & 1997 & 7 (6 signalisert) & 0,24 & 0,12 & 0,10 \\
\hline $5 / 6$ & 1998 & 8 (4 signalisert) & 0,16 & 0,10 & 0,26 \\
\hline $245 / 246$ & 1999 & 5 (4 signalisert) & 0,22 & 0,25 & 0,03 \\
\hline
\end{tabular}




\subsubsection{Scanner}

Scanner konvertieren analoge Luftbilder in eine digitale Form. Hinsichtlich der Wahl des Scanners und der räumlichen Auflösung sind in Abhängigkeit des Auswertungszieles und der Genauigkeitsanforderungen Entscheidungen zu treffen. Für die Digitaliserung wurde der fotogrammetrische Scanner RM2 von Wehrli \& Associate Inc. und ein A3 Büroflachbettscanner Mustek Paragon 1200 eingesetzt. Für eine hohe geometrische und radiometrische Genauigkeit ist die Verwendung eines fotogrammetrischen Scanners vorteilhaft. Der RM2-Scanner korrespondiert mit dem Aufbau eines analytischen Stereoplotters und besteht aus einer Bildträgerplatte, einem Filterrad mit einer RGB-Auswahl des spektralen Bereiches, einem Linsensystem (Rodenstock) und einem TDI- (Time delay and integration sensor) Sensor. Der TDI-Sensor ist ein Flächensensor mit $2048 \times 96$ Elementen. Die kontinuierlichen elektromagnetischen Signale werden mit einer radiometrischen Auflösung von 12 bit integriert und auf 8 bit mit einer konfigurierbaren Farbtabelle komprimiert. Die Farbauszüge Blau, Grün, Rot werden in drei Durchgängen digitalisiert. Nach Kalibirierung des Scanners wird eine geometrische Genauigkeit von $3 \mu \mathrm{m}$ erreicht. Das Digitalisierungsintervall für Filmvorlagen kann mit 12, 24, 48 oder $96 \mu$ m ausgewählt werden.

Der Mustek Flachbettscanner hat eine maximale geometrische Auflösung von 1200 $\times 600$ dpi. Ein Zeilensensor scannt die Farbauszüge der Papier- oder Filmvorlagen in einem Durchgang. Für den Vergleichstest wurde mit einer Auflösung von 600 dpi (entspricht $48 \mu m$ ) digitalisiert.

In einer vergleichenden Untersuchung wurde der Einfluss der räumlichen Auflösung des Scanners auf die Genauigkeit digitaler Höhenmodelle untersucht (Kap. 3.3.5).

\subsubsection{Digitale Fotogrammetrische Systeme (DFS)}

Digitale Fotogrammetrische Systeme, im Folgenden DFS genannt, verwenden digitale Bilder im Gegensatz zu den analogen Luftbilddiapositiven. Die digitalen Bilder können aus gescannten Luftbildern entstehen. Es können aber auch Bilder aus direkten digitalen Aufnahmen von flugzeug- und satellitengetragenen Sensoren verarbeitet werden (z. B. Aster, IRS, Ikonos, Spot, Quickbird). Ein DFS besteht aus einem Computer mit hoher Rechnerleistung und Festplattenkapazität, großem Arbeitsspeicher und einem Software-System. Die Software ist modular aufgebaut und übernimmt die gleichen Funktionen wie analytische Stereoplotter. So können fotogrammetrische Aufgaben wie Bildorientierung mit Bündelausgleichung, Berechnung und Visualisierung von digitalen Höhenmodellen, Generierung und Mosaikbildung von Orthofotos und Vektordatenerfassung in einer CAD (Computer-Aided-Design)-Umgebung erledigt werden.

Eine Stereobetrachtung in Farbe ist beim DFS „SoftPlotter“ auf einem Bildschirm mit Wechselblendentechnik möglich. Auf dem Bildschirm werden abwechselnd linke und rechte Bilder mit einer Frequenz von $120 \mathrm{~Hz}$ präsentiert. Über eine Infrarotsteuerung wird das Auf- und Abblenden der rechten und linken Seite einer speziellen Flüssigkristallbrille geregelt. Das rechte Bild wird mit dem rechten Auge und das linke Bild mit dem linken Auge gesehen, wodurch ein räumlicher Stereoeffekt entsteht. In 
einem CAD-Programm können dreidimensionale Messungen mit einer Maus oder anderen Eingabemöglichkeiten wie bei einem analytischen Plotter durchgeführt werden. Die wichtigsten Vorteile der DFS gegenüber analytischen Auswertungssystemen sind die automatische Erzeugung von digitalen Höhenmodellen, die digitale Orthofotoherstellung und die halbautomatische Bildorientierung (Heipke 1995).

\subsubsection{Digitale Höhenmodelle}

Ein digitales Höhenmodell (DHM) ist eine digitale und mathematische Repräsentation eines existierenden oder virtuellen Objektes und seiner Oberfläche (Paparoditis und Polidori 2002). Wenn die Information auf Bodenerhebungen beschränkt ist, spricht man von einem digitalen Geländemodell (DGM), wenn die Information die höchsten Punkte enthält, bezeichnet man diese als digitales Oberflächenmodell (DOM). Die Differenz von DGM und DOM wird als normalisiertes digitales Oberflächenmodell bezeichnet (NDOM).

\section{Digitales Oberflächenmodell (DOM)}

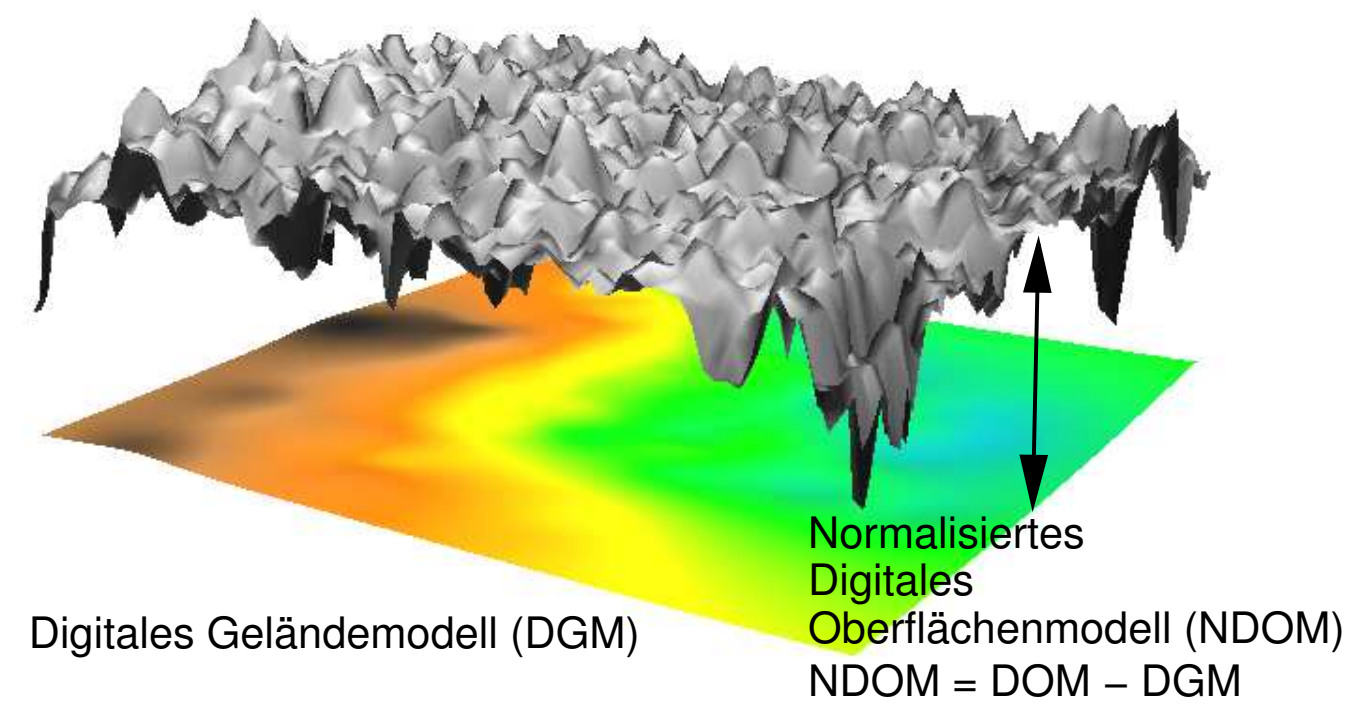

Abb. 3.6: Digitales Oberflächenmodell, Geländemodell und normalisiertes Oberflächenmodell der Eichen-Fläche 502

Für einen Vergleich der Genauigkeit der digitalen Höhenmodelle wurden drei verschiedene digitale fotogrammetrische Auswertungssysteme (DFS) eingesetzt: Das DFS „SoftPlotter“ (Version 1.8.3, Autometric Inc. 1998) ist ein einzeln stehendes SoftwareSystem, das alle in Kap. 3.3.2.4 beschriebenen Funktionen übernehmen kann.

Die DFS „OrthoEngine“ (PCI Geomatics 2001) und „OrthoBASE“ sind innerhalb der Bildverarbeitungssysteme PCI Geomatica 8.0 und ERDAS Imagine 8.5 integriert 
und bieten ebenfalls Funktionen zur Erzeugung von digitalen Höhenmodellen an. Eine Stereobetrachtung und die Erzeugung von 3D-Vektordaten ist mit Hilfe des Anaglyphenverfahrens realisiert. In allen drei Systemen können auch digitale Bilder satellitengestützter Sensoren verarbeitet werden. Die für die DHM-Ableitung verwendeten Algorithmen der Bildzuordnung sind nicht veröffentlicht. Es können einige allgemeine Merkmale der Bildzuordnungsalgorithmen beschrieben werden.

Alle drei Systeme verwenden für die DHM-Generierung die Methode der flächenbasierten Bildzuordnung. In der Literatur werden zwei Verfahren unterschieden: die Kreuzkorrelation und die Kleinste-Quadrate-Bildzuordnung. Bei der Kreuzkorrelation dienen kleine Bildausschnitte der Größe von 5 x 5 bis 15 x 15 Pixel als Grundelemente der Zuordnung korrespondierender Punkte. Das Zentrum der Matrix wird als Position des Zuordnungspunktes verwendet. Die Bildzuordnung wird durchgeführt, indem eine Matrix A (Musterfenster) der Größe von 5 x 5 Bildelementen um ein gegebenes Pixel im linken Bild betrachtet wird (s. Abb. 3.7). Eine Suche nach der korrespondierenden Matrix B im rechten Bild (Zuordnungsfenster) wird durchgeführt, indem eine Matrix der gleichen Größe wie Matrix A über einen Suchbereich des rechten Bildes geschoben wird. Der Suchbereich im rechten Bild ist größer als das Musterfenster im linken Bild, da die exakte Position des Zuordnungspunktes im rechten Bild zunächst nicht bekannt ist. Für jede Position des Zuordnungsfensters im rechten Bild wird der Kreuzkorrelationskoeffizient $^{1}$ aus den Grauwerten der Matrix A und B berechnet. Er berechnet sich nach der folgenden Formel (Wolf und Dewitt 2000):

$$
c=\frac{\sum_{i=1}^{m} \sum_{i=1}^{n}\left[\left(A_{i j}-\bar{A}\right)\left(B_{i j}-\bar{B}\right)\right]}{\sqrt{\left[\sum_{i=1}^{m} \sum_{j=1}^{n}\left(A_{i j}-\bar{A}\right)^{2}\right]\left[\sum_{i=1}^{m} \sum_{j=1}^{n}\left(B_{i j}-\bar{B}\right)^{2}\right]}}
$$

wobei

$c \quad=$ Korrelationskoeffizient

$m \quad=$ Anzahl der Zeilen der Matrix

$n \quad=$ Anzahl der Spalten der Matrix

$A_{i j}=$ Grauwerte der Mustermatrix A in Zeile $\mathrm{i}$, Spalte $\mathrm{j}$

$\bar{A} \quad=$ Mittelwert der Grauwerte der Matrix A

$B_{i j}=$ Grauwerte der Zuordnungsmatrix B in Zeile $\mathrm{i}$, Spalte $\mathrm{j}$

$\bar{B} \quad=$ Mittelwert der Grauwerte der Matrix B

Der Korrelationskoeffizient kann Werte von -1 bis +1 annehmen. Die Werte -1 oder +1 bezeichnen eine perfekte Korrelation und eine exakte Bildzuordnung. Nachdem der Korrelationskoeffizient für alle möglichen Positionen im Suchfenster berechnet wurde, entsteht eine Korrelationsmatrix C. Liegt das Maximum dieser Korrelationsmatrix über

\footnotetext{
${ }^{1}$ auch Produktmoment-Korrelationskoeffizient genannt
} 
einem Schwellenwert, kann der jeweilige Punkt zugeordnet werden (Kasser und Egels 2001, Al-Rousan et al. 1997, Kraus 1997, Heipke 1996).
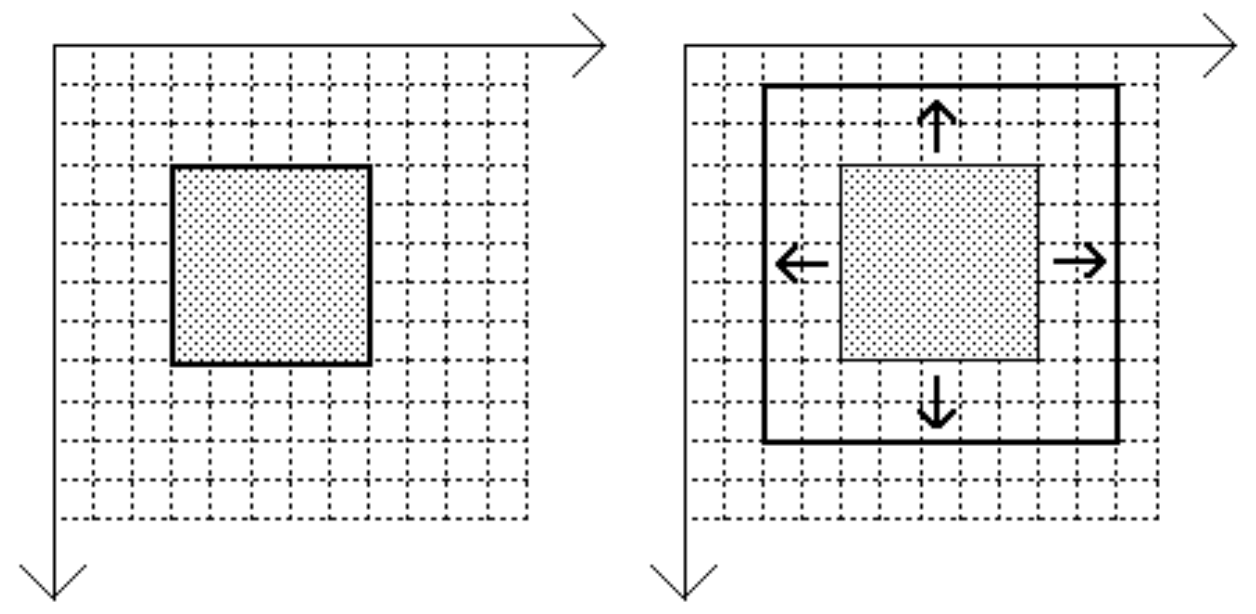

Abb. 3.7: Musterfenster (linkes Bild) und Zuordnungsfenster mit Suchmatrix (rechtes Bild) (aus Heipke 1996)

Eine Verbesserung der Bildzuordnung kann durch einen weiteren Ansatz zur flächenbasierten Bildzuordnung erreicht werden. Bei der Methode der Kleinsten-QuadrateZuordnung (Least-Squares-Matching, auch LSM genannt) wird ein kleiner Ausschnitt im Referenzbild vorgegeben. Dieser Ausschnitt wird geometrisch in ein anderes Bild eingepasst. Hierfür wird eine affine Transformation eingesetzt, wobei das Oberflächenelement als Ebene angesehen wird. In mehreren Rechenschritten wird die Summe der Abweichungsquadrate minimiert. Als Beobachtungen dienen die Unterschiede zwischen den Grauwerten beider Bilder. Herleitung des mathematischen Modells und Hinweise für eine programmtechnische Umsetzung werden z. B. in Wolf und Dewitt (2000) und Förstner (1982) gegeben. Der Vorteil des LSM ist die hohe Lagegenauigkeit von 0,1 bis 0,3 Pixel. Es sind allerdings sehr genaue Näherungswerte der Positionen der Fenster notwendig, damit die Punkte zugeordnet werden können.

Um die Rechenzeit zu verkürzen, wird die Suche auf eine einzige Linie begrenzt, indem das Prinzip der epipolaren Geometrie als zusätzliche Bedingung eingeführt wird. Die relative Orientierung von zwei Luftbildern zum Zeitpunkt der Aufnahme muss bekannt sein. Eine epipolare Ebene eines Objektpunktes wird im dreidimensionalen Raum zwischen den Projektionszentren und diesem Punkt aufgespannt. Epipolare Linien werden im linken und rechten Bild berechnet. Um die Bildzuordnung zu vereinfachen, werden die Bilder in einem Vorverarbeitungsschritt in den Normalfall der Stereoauswertung transformiert, indem die y-Parallaxen im gesamten Stereomodell beseitigt werden. Die Bildzuordnung braucht dann nur noch auf einer Linie in Richtung der Bildbasis durchgeführt werden (Heipke 1996).

Eine weitere Methode zur Steigerung der Effizienz der Algorithmen ist die Verwendung von Bildpyramiden. Die Luftbilder werden in mehrenen Auflösungen um den Fak- 
tor 2 reduziert. Die Bildzuordnung wird zunächst mit der geringsten Auflösung durchgeführt. Die Ergebnisse dienen dann als Näherungen für die nächst höhere Auflösung.

Die Systeme unterscheiden sich hinsichtlich der Wahlmöglichkeiten zur Steuerung der Parameter der Bildkorrelation. Während OrthoEngine nur drei Strategien anbietet, stellen SoftPlotter und OrthoBase eine Vielzahl zusätzlicher Einstellungsparameter bereit. Die Ergebnisse der Bildzuordnung werden in einem weiteren Prozess gefiltert und fehlende Werte interpoliert. SoftPlotter und OrthoEngine ermöglichen es darüber hinaus, diese Funktion auszuschalten. Die Handbücher der drei Software-Hersteller bieten nur wenige konkrete Hinweise für die Auswahl der optimalen Einstellungen und beschreiben die verwendeten Algorithmen nur im Allgemeinen (Baltsavias und Käser 1998). Anwender sind auf eigene empirische Tests angewiesen, um die Einstellungen zu optimieren (vgl. Gooch und Chandler 2001).

\subsubsection{Digitale Orthofoto-Herstellung}

Ein Orthofoto ist ein differentiell entzerrtes, von der Zentralperspektive in eine orthogonale Projektion umgewandeltes Luftbild (Hildebrandt 1996). Es verbindet die Vorteile der Geometrie einer Karte mit dem Informationsgehalt eines Luftbildes. In Orthofotos können Strecken und Winkel wie in einer Karte gemessen werden und eignen sich sehr gut für eine Integration in Geoinformationssystemen. Sie werden häufig als Referenz verwendet, wenn Geodaten unterschiedlicher Qualität zusammengeführt werden. In der vorliegenden Arbeit wurden Orthofotos für eine rechnergestützte, digitale Auswertung eingesetzt.

Für die Herstellung der Orthofotos mit Mitteln der digitalen Fotogrammetrie müssen die digitalen Originalluftbilder, die absoluten Orientierungsdaten (s. Kap. 3.3.2.2) und ein digitales Höhenmodell vorliegen.

\subsubsection{Datenverarbeitung}

Für die Auswertung der Ergebnisse des visuellen Verfahrens wurde die Software SPlus 4.5 und MS Excel eingesetzt. Die Ergebnisse der Interpretation sowie die Lagekoordinaten der Kronenrandpunkte und der Kronenspitzen waren Datengrundlage für die kartografischen Darstellungen mit Arc/Info 8.0.1 Workstation und ArcView 3.1. Die Ergebnisse der Kronenmessungen des visuellen Auswertungsverfahrens wurden mit dem Programm KRONE erstellt (Heydecke und Fuchs 2000).

Zur weiteren Entwicklung von Programmen und zur Durchführung von Analysen wurde Software eingesetzt, die der Freien Software-Lizenz ${ }^{2}$ des GNU-Projektes (GNU

\footnotetext{
${ }^{2}$ „Free software is a matter of the users' freedom to run, copy, distribute, study, change and improve the software. More precisely, it refers to four kinds of freedom, for the users of the software:

- The freedom to run the program, for any purpose.

- The freedom to study how the program works, and adapt it to your needs.

- The freedom to redistribute copies so you can help your neighbor.

- The freedom to improve the program, and release your improvements to the public, so that the whole community benefits." (GNU project 2002)
} 
GPL, General Public Licence, GNU project 2002) und der Open-Source-Initiative (OSI 2002) unterliegen. Die einzelnen Komponenten werden im Folgenden beschrieben (Abb. $3.8)$.

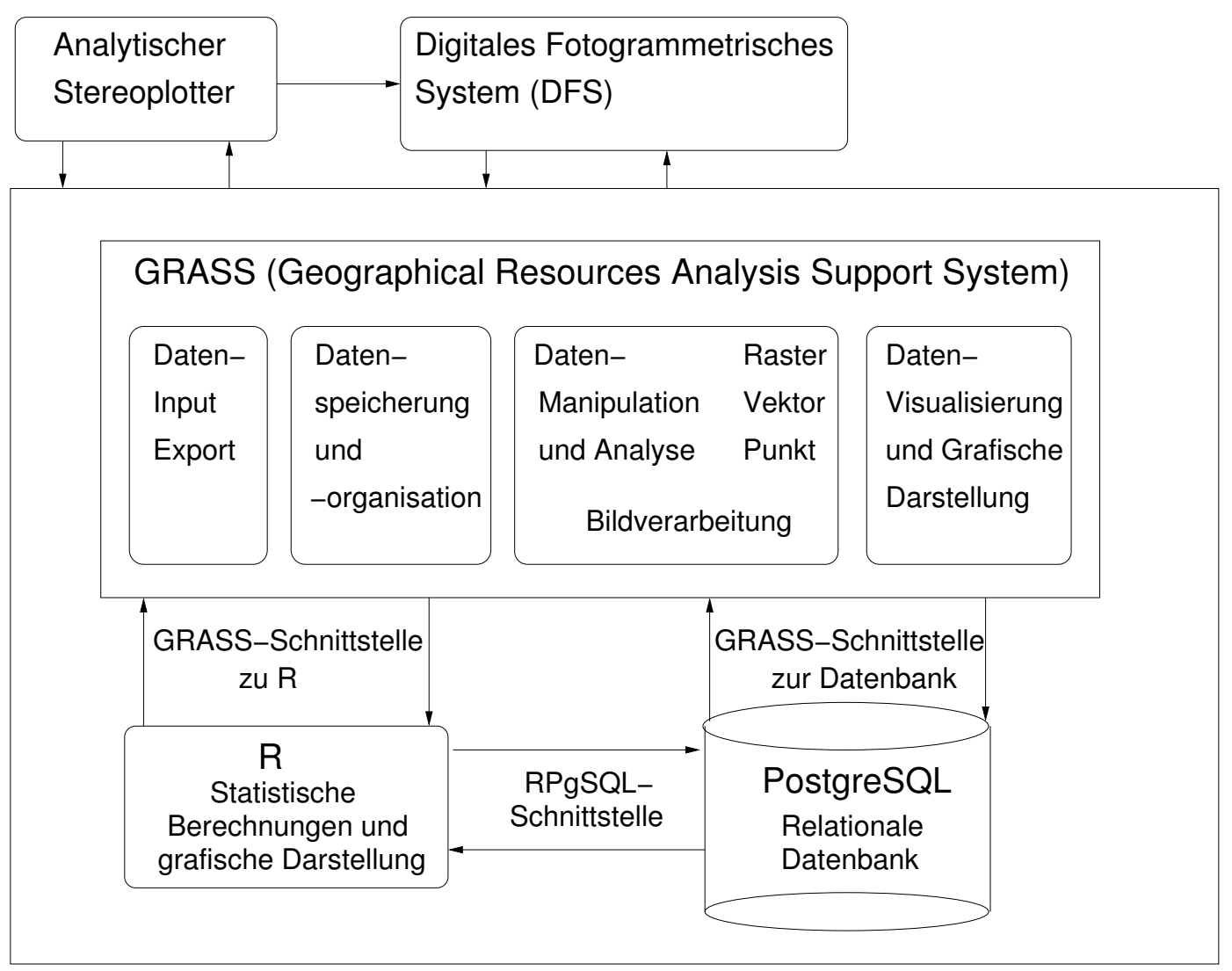
Abb. 3.8: Übersicht über die verwendeten Software-Werkzeuge zur weiteren Datenver-
arbeitung

Geoinformationssystem und Bildverarbeitung. Zentrale Bedeutung nimmt das geografische Informationssystem GRASS (Geographical Resources Analysis Support System) (GRASS Development Team 2002) ein (Abb. 3.8). GRASS wurde ursprünglich von einem Forschungslabor der US-Armee (CERL) in Champain, Ilinois, zur Unterstützung der Landnutzungsplanung von Militärstandorten im Zeitraum von 1982 - 1995 entwickelt. Seit 1999 wird GRASS unter der GNU GPL (General Public License) weiterentwickelt. Das Internet spielt eine Hauptrolle in der Koordinierung und Unterstützung der Software-Entwicklung. GRASS bietet grundlegende Funktionen eines geografischen Informationssystemes wie Raster- und Vektordatenverarbeitung, Bildverarbeitungs- und grafische Visualisierungsmodule (Neteler und Mitasova 2002, Neteler 2000). 
Relationale Datenbank. Da in der internen Datenbankstruktur von GRASS bisher nur ein Attribut abgelegt werden kann, wird die Datenspeicherung durch die relationale Datenbank PostgreSQL (PostgreSQL Global Development Group 2002) ergänzt.

Statistik-System. Eine Erweiterung der statistischen Funktionen mit dem Statistik-System R (R Project 2002) erleichtert eine explorative Analyse und Präsentation der Ergebisse. Die statistische Datenanalyse basiert auf modernen Programmierungskonzepten, die die Integration von Skripten mit kompilierten dynamisch geladenen Bibliotheken erlauben (Venables und Ripley 2000).

\subsubsection{Luftbildmerkmale}

Das Handbuch der Europäischen Kommission zur Anwendung der Fernerkundung für die Waldzustandserfassung definiert Schlüsselvariablen für Erhebungen im Rahmen des Level 2-Programmes (Tab. 3.8) (WGRSA 2000).

Tab. 3.8: Einzelbaum- und Bestandesmerkmale für die Erfassung mittels Fernerkundung auf Level 2-Flächen

\begin{tabular}{l|l}
\hline Einzelbaummerkmale & Bestandesmerkmale \\
\hline 3-D-Position (x,y,z) & Abgrenzung der Untersuchungsfläche \\
Baumhöhe & Repräsentanz \\
Kronenlänge & natürliche Altersklasse \\
Kronenpolygon & Überschirmungsgrad \\
Kronenmodell & Vitalitätszustand des Bestandes \\
Kronenzustand & Bewirtschaftungspraxis \\
Vergilbung & digitales Bestandesoberflächenmodell \\
soziale Stellung & digitales Geländemodell \\
Besonderheiten & \\
\hline
\end{tabular}

Mit der geringsten Auswertungsintensität sind Beschreibungen des Bestandes vorgesehen. Es werden Lage und Abgrenzung der Untersuchungsflächen dokumentiert und eine qualitative Einschätzung der Flächen im Vergleich zur nahen Umgebung getroffen. Die Einschätzung wird anhand der Artenzusammensetzung, der natürlichen Altersklasse, des Überschirmungsgrades, der Bewirtschaftungspraxis und des Vitalitätszustandes getroffen. Jedes dieser Merkmale wird in drei Klassen unterteilt: 1. vergleichbar, 2. ähnlich und 3. verschieden. Hierzu ist anzumerken, dass der Begriff „,nahe Umgebung“ nicht ausreichend definiert ist. Zum anderen sind die aufgeführten Bestandesmerkmale komplex und lassen sehr viel Subjektivität bei der gutachtlichen Einstufung zu. Das Er- 
messen des Interpreten ist auch beim Vergleich des Vitalitätszustandes zu groß, da die Klassengrenzen nicht eindeutig definiert werden.

Die wichtigsten einzelbaumbezogenen Merkmale sind Kronenzustand und Vergilbung, die mit visueller Interpretation unter Verwendung der baumartenspezifischen Schlüssel bewertet werden. Zusätzlich sind Kronenmessungen mit fotogrammetrischen Auswertungssystemen möglich. Der lagetreuen Erfassung und Zuordnung der Einzelbäume wird eine hohe Bedeutung zugemessen, da diese erst eine Verknüpfung zu anderen Erhebungen auf den Untersuchungsflächen ermöglicht und die Voraussetzung für Folgeaufnahmen darstellt.

\subsubsection{Interpretationsschlüssel}

Interpretationsschlüssel sind Auswertungsanweisungen, die die Grundlage einer visuellen Luftbildauswertung bilden. Sie stellen die Beziehungen zwischen den terrestrischen und luftbildsichtbaren Merkmalen her und ermöglichen eine Beurteilung nach einheitlichen Kriterien. Sie sind die Grundlage für Schulungsprogramme von Luftbildinterpreten in größeren Inventurprojekten.

In der vorliegenden Arbeit wurde ein allgemeiner Interpretationsschlüssel für die Baumarten Fichte, Buche und Eiche eingesetzt. Dieser Schlüssel wurde von der Arbeitsgemeinschaft Forstlicher Luftbildinterpreten (AFL) bereits 1993 als Richtlinie beim Verein Deutscher Ingenieure (VDI ) (AFL/VDI 1993) veröffentlicht und ist in das aktuelle EU-Handbuch (WGRSA 2000) übernommen worden. Er ist ein Beispielschlüssel, der Gestalt- und Farbmerkmale beschreibt, die mit Stereogrammen und Aufsichtzeichnungen veranschaulicht werden. Als Gestaltmerkmal wird die Form der Krone definiert. Sie ist die größte Einheit der geometrischen Merkmale und beschreibt die äußeren Konturen im Um- und Aufriss. Die Grobstruktur ist eine mittlere Einheit zur Strukturbeschreibung innerhalb der Krone. Die Feinstrukur ist die kleinste Einheit zur Beschreibung von Ästen und Blattgruppen. Die Farbmerkmale sind aufgrund der Schwankungen der Farbwiedergabe im CIR-Film nur allgemein definiert. Die Farbbeschreibung lehnt sich an das HSI-Farbsystem (Hue, Saturation, Intensity) an und charakterisiert Farbton, Sättigung und Helligkeit. Mit der Farbverteilung werden Farbschwankungen innerhalb der Baumkrone bewertet und als Texturmerkmal definiert.

Die allgemeinen Merkmale des AFL-Schlüssels sollten durch zusätzliche Geländeerhebungen angepasst werden, die im idealen Fall zeitnah zur Befliegung realisiert werden. Bei den vorliegenden Untersuchungen war dies nicht möglich, da die Luftbildauswertungen nicht im gleichen Jahr wie die Befliegungen durchgeführt wurden. Die Identifizierung von Einzelbäumen war mit Hilfe von Stammverteilungsplänen und Kronenkarten möglich, an denen jährlich eine terrestrische Einschätzung des Nadel/ Blattverlustes in $5 \%$-Stufen durchgeführt wurde. Diese Bäume mit bekannter Einstufung im Gelände wurden in einer Lernphase des Interpreten mit dem allgemeinen Merkmalen des AFL-Schlüssels verglichen. Zusätzliche Bäume, deren Einstufung dem Interpreten nicht bekannt war, dienten zur Überprüfung der Interpretationsergebnisse. 


\subsubsection{Segmentierung}

Segmentierung ist die Zusammenfassung von Bildpunkten zu größeren Einheiten. Nach Maßgabe quantitativer Merkmale (z. B. Grauwert oder Textur) werden Objekte in einem digitalen Bild vom Hintergrund isoliert. In Anwendungsbereichen der digitalen Bildverarbeitung und Mustererkennung ist dies ein wesentlicher Schritt in Richtung einer automatischen Erkennung von Objekten. Anschließend können Eigenschaften der Segmente beschrieben und zu sinnvollen Objekten zusammengesetzt werden (Haberäcker 2000, Kraus 1990).

In dieser Arbeit werden einzelne Baumkronenpolygone als Segmente einer visuellen stereoskopischen Abgrenzung der Kronenränder definert. Dieses Vorgehen stellt bisher das zuverlässigste Verfahren dar. Die menschliche Wahrnehmung ist auf die Erkennung flächenhafter Strukturen spezialisiert. Das Augen- und Gehirnsystems des Menschen ist in dieser Hinsicht sehr flexibel und leistungsfähig. So können bestimmte Muster auf einem Luftbild sicher erkannt werden, auch wenn Beleuchtung des Objektes, Bildmaßstab und Qualität der Abbildung stark variieren (Albertz 1998).

Auf Grundlage der Kronenpolygone können statistische Kennwerte wie der arithmetische Mittelwert und die Standardabweichung verschiedener digitaler Attribute der Bildpunkte innerhalb der Segmente berechnet werden.

Zur automatischen Segmentierung von digitalen Bildern wurden eine Vielzahl von Algorithmen entwickelt. Gonzalez und Woods (2002) unterscheiden folgende Methoden:

1. Kanten und Linienextraktion,

2. Kantenverbindung (Edge Linking),

3. Schwellenwertverfahren,

4. Gebietswachstum (Region Growing),

5. und das morphologische Wasserscheidenverfahren.

Das Wasserscheidenverfahren ist ein erfolgversprechendes Verfahren für die Delinierung von Einzelbäumen. Böttcher und Kleinn (2003), Ziegler et al. (2000), Schardt et al. (2002) verwenden für diesen Zweck hochauflösende Oberflächenmodelle aus Laserscannerdaten, Wang et al. (2002) verarbeiten multispektrale CASI-Aufnahmen. In einem Experiment wurde die Verwendung eines fotogrammetrischen digitalen Oberflächenmodells getestet (Kap. 4.3).

Das Grundprinzip des Wasserscheidenverfahrens besteht in der Verwendung eines digitalen Höhenmodells (DHM) aus diskreten Punkten, das ein Landschaftsmodell repräsentiert. Dieses Landschaftsmodell wird virtuell mit Niederschlägen überflutet. Fallen Niederschläge auf diese Landschaft, fließt ein Regentropfen gemäß der Gravitation den steilsten Weg bergab, bis er ein lokales Minimum erreicht. Eine andere Vorstellung geht davon aus, dass eine Landschaft untergetaucht wird, wobei das ansteigende Wasser durch Löcher in lokalen Tiefpunkten aufsteigt (Gonzalez und Woods 2002, Roerdink 
und Meijster 2001). Das Hauptziel der Segmentierungsalgorithmen besteht darin, Wasserscheiden zu finden. Wasserscheiden bilden die Trennlinien des Wasserabflusses innerhalb der Regionen. Es werden drei Klassen von Punkten unterschieden: (a) Punkte, die zum Minimum einer Region gehören, (b) Punkte, von denen aus ein Tropfen Wasser mit Sicherheit zu einem einzigen Minimum fließt und (c) Punkte, von denen aus das Wasser in Richtung mehrerer Minima fließt. Die Menge der Punkte, die die Bedingung (b) erfüllen, werden Einzugsgebiete genannt, die Punkte, die Bedingung (c) erfüllen, sind die Trennlinien oder Wasserscheiden.

Ein sehr effizienter Algorithmus für diesen Zweck der topographischen Analyse ist das Programm Terraflow, das an der Duke University, Durham, NC, entwickelt wurde (Arge et al. 2001). Es wurde in das Geoinformationsystem GRASS implementiert (r.terraflow).

Das Prinzip des Fließwege-Algorithmus (Flow Routing) besteht in dem Überfluten des Geländes und der Zuordnung der Fließrichtung. Dieses wird in vier Teilschritten erreicht:

(a) es werden flache Gebiete (Plateaus) und Senken identifiziert; (b) die Fließrichtungen auf den Plateaus werden zugeordnet; (c) das Gelände wird überflutet und (d) die Fließrichtungen werden berechnet.

Zur Berechnung der Fließrichtung wird ein Fenster der Größe von $3 \times 3$ Pixel (acht Nachbarelemente um ein zentrales Element (Pixel)) bestimmt. Ein Nachbar des zentralen Pixels wird „Bergab-Nachbar“ oder „Bergauf-Nachbar“ genannt, wenn er eine geringere bzw. größere Höhe als das zentrale Pixel aufweist. Der Gradient des zentralen Pixels zu einem seiner Nachbarn wird als das Verhältnis zwischen der Höhendifferenz der Pixel und der zwischen ihnen liegenden horizontalen Distanz definiert. Der Gradient des Zentralpixels ist positiv hinsichtlich der „Bergauf-Nachbarn und Null hinsichtlich der Nachbarn mit der gleichen Höhe. Es wird nun nach der Fließrichtung mit dem höchsten Gradienten, d. h. mit dem steilsten „Bergab-Nachbarn“ gesucht.

Es gibt zwei Methoden zur Berechnung der Fließrichtungen: die Einweg-Fließrichtungsmethode, auch D8 genannt, (Wasser fließt in eine einzige Richtung zum Nachbarn mit dem höchsten Gefälle) und die Mehrwege-Fließrichtungsmethode, wobei das Wasser in mehrere Richtungen zu allen Nachbarn mit Gefälle fließt.

Zur Lösung des Problems der automatischen Abgrenzung von Baumkronen mittels des Wasserscheidenverfahrens sind mehrere Vorverarbeitungsschritte durchzuführen: 
1. Ein normalisiertes digitales Oberflächenmodell wird invertiert, d. h. die Spitzen der Kronen werden als Tiefpunkte oder Senken definiert (z. B. mit r.recode in GRASS). Das invertierte Kronenoberflächenmodell wird als Landschaft interpretiert, die Kronenränder stellen Trennlinien zwischen den Einzugsgebieten dar.

2. Das Höhenmodell wird mit einem Tiefpassfilter geglättet. Gaußsche Filterkerne der Größe $m \times m$ werden aus einer diskretisierten, zweidimensionalen Gaußfunktion mit verschiedenen Standardabweichungen abgeleitet. Die Dichte einer zweidimensionalen Normalverteilung wird mit der folgenden Formel berechnet (Haberäcker 2000):

$$
g(x, y, \sigma)=\frac{1}{2 \pi \sigma^{2}} e^{-\frac{x^{2}+y^{2}}{2 \sigma^{2}}}
$$

wobei

$$
\begin{aligned}
& \mathrm{x}, \mathrm{y}=\text { Pixelkoordinaten eines bewegten Fensters mit dem Zentralpixel }(0,0) \\
& \sigma \quad=\text { Standardabweichung. }
\end{aligned}
$$

Filterkerne verschiedener Größen und Standardabweichungen wurden mit dem Modul r.mapcalc in GRASS definiert und zur Glättung der invertierten Höhenmodelle eingesetzt.

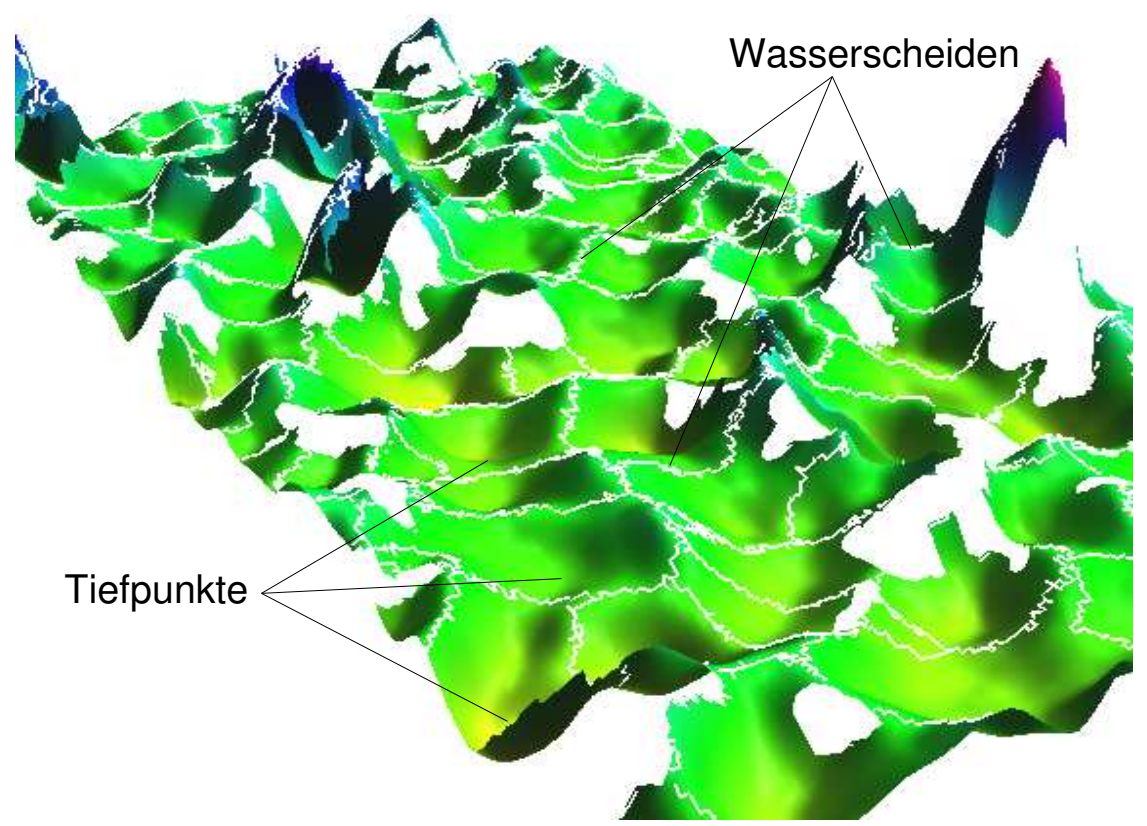

Abb. 3.9: Invertiertes normalisiertes Oberflächenmodell eines Eichen-BuchenMischbestandes mit Tiefpunkten (Kronenspitzen) und Wasserscheiden (Kronenränder) 


\subsubsection{Spektrale Merkmale}

RGB-Kanäle des digitalen Orthofotos. Ein digitales Farbinfrarot-Orthofoto besteht aus drei Farbauszügen oder Kanälen der Spektralbereiche Grün, Rot und nahes Infrarot, denen die additiven Farben Blau, Grün und Rot zugeordnet werden. Die Bezeichnung der Kanäle richtet sich im Folgenden nach der spektralen Sensitivität.

Normalisierter Differenz-Vegetationsindex (NDVI). Der NDVI ist einer der am häufigsten verwendeten Indices, die in der Fernerkundung zur Charakterisierung der Vegetation eingesetzt werden (Bannari et al. 1995). Die Kombination der Reflexionssignale des roten und infraroten Spektralbereiches erlaubt die Trennung von aktiv Fotosynthese betreibenden Pflanzen und Nicht-Vegetation (Tucker 1979). Die Verwendung von Differenzen und Summen von Bändern verringert den Einfluß von atmosphärischen, bildortabhängigen und topographischen Störeinflüssen und wird deshalb in Zeitreihenvergleichen bevorzugt (Mather 1999). Während der Zusammenhang zwischen Chlorophyllgehalt und NDVI nicht sehr eng ist, haben mehrere Autoren eine Beziehung zur grünen Biomasse und zum Blattflächenindex (LAI) nachgewiesen (Spanner et al. 1990).

Als Ratiokanal wurde der „Normalized Difference Vegetation Index“ (NDVI) als Fließkommazahl nach der Formel berechnet (r.mapcalc in GRASS):

$$
N D V I=\frac{(N I R-R O T)}{(N I R+R O T)}
$$

Eine Transformation auf eine 8 Bit-Auflösung (Grauwerte von 0 bis 255) wurde nach Mather (1999) durchgeführt :

$$
N D V I_{i j}^{\prime}=\frac{N D V I_{i, j}-N D V I_{\min }}{N D V I_{\max }-N D V I_{\min }} \cdot 255
$$

wobei

$i \quad=$ Spalten der NDVI-Matrix,

$j \quad=$ Zeilen der NDVI-Matrix,

$N D V I_{\min }=$ minimaler Wert der NDVI-Matrix,

$N D V I_{\max }=$ maximaler Wert der NDVI-Matrix.

Differenzkanäle. Als Differenzkanal wurde der Index

$$
V I_{d i f f}=G R \ddot{U} N-R O T
$$

(z. B. Tucker 1979) für die Erkennung der abgestorbenen Bäume genutzt.

Hauptkomponenten. Die Hauptkomponentenanalyse ist eine der wichtigsten Techniken der statistischen Faktorenanalyse (Bortz 1999) und wird in der Fernerkundung zur Komprimierung und Verbesserung der Klassifikation von hyper- und multispektralen Fernerkundungsdaten eingesetzt (Schowengerdt 1997, Jensen 1996). Die Kanäle der 
digitalisierten CIR-Luftbilder korrelieren meist stark miteinander. Durch die Extraktion der Hauptkomponenten entstehen neue synthetische Kanäle, die voneinander unabhängig sind und sukzessiv maximale Varianz erklären. In der vorliegenden Arbeit wurde geprüft, ob die erste Hauptkomponente der digitalisierten CIR-Luftbilder die automatischen Bildzuordnungen bei der Berechnung der digitalen Oberflächenmodelle verbessern kann (Kap. 3.3.5). Die zweite Hauptkomponente der digitalen Orthofotos wird zur Klassifikation der Vitalität eingesetzt (s. Kap. 4.7, Berechnung mit i.pca in GRASS). Die erste Hauptkomponente der Lagekoordinaten der Kronenrandpunkte dient zur Bestimmung der Hauptorientierung von Baumkronen und Lücken mit Schatten.

Textur. Textur ist ein wichtiges visuelles Merkmal, das durch die räumliche Verteilung von Farbvariationen innerhalb relativ kleiner Bildbereiche entsteht. Obwohl Texturen große Bedeutung für die Analyse von fotografischen und multispektralen Bildern haben, gibt es keine präzisen Definitionen (vgl. Haralick und Shapiro 1992, Van Gool et al. 1985). Zur Charakterisierung der Textur digitaler Bilder werden verschiedene statistische Verfahren angewendet, die lokale Bildeigenschaften beschreiben. Häufig wird eine Grauwertübergangsmatrix (Cooccurrence-Matrix) eingesetzt, die auf der Schätzung von bedingten Wahrscheinlichkeitsfunktionen beruht. Die relativen Häufigkeiten des Auftretens einer Grauwertkombination bezüglich einer Relation werden in einer symmetrischen Matrix berechnet und sind Funktionen des Winkels zwischen benachbarten Grauwerten und der Distanz. Von den 14 klassischen Texturmerkmalen, die Haralick et al. (1973) vorstellen, wurden die Merkmale contrast (con) und variance (var) ausgewählt und für einzelne Baumkronenpolygone mit dem GRASS-Modul i.texture berechnet. Contrast ist mit der mittleren Grauwertdifferenz zwischen Nachbarpixeln assoziiert, Variance ist ein Maß für die mittlere Grauwertdifferenz zwischen Pixeln und ihrem Mittelwert:

$$
\begin{aligned}
\text { con } & =\sum_{i=0}^{N g-1} \sum_{j=0}^{N g-1}(i-j)^{2} \cdot g(i, j) \\
\operatorname{var} & =\sum_{i=0}^{N g-1} \sum_{j=0}^{N g-1}(i-\mu)^{2} \cdot g(i, j)
\end{aligned}
$$

wobei

$i=$ Spalten der Cooccurrence-Matrix,

$j \quad=$ Zeilen der Cooccurrence-Matrix,

$N_{g} \quad=$ Anzahl der Grauwerte in einem Bild (bzw. Bildsegment),

$g(i, j)=$ Auftrittswahrscheinlichkeit der Pixel-Paar-Kombinationen,

$$
\begin{aligned}
& =p(i, j) / \sum_{i=0}^{N_{g}-1} \sum_{j=0}^{N_{g}-1} p(i, j) \\
\mu & =\sum_{i=0}^{N_{g}-1} \sum_{j=0}^{N_{g}-1} i \cdot g(i, j) .
\end{aligned}
$$


Contrast nimmt relativ geringe Werte an, wenn die höchsten Werte der CooccurrenceMatrix in der Nähe der Hauptdiagonalen liegen, weil die Differenzen $i-j$ hier am kleinsten sind. Die Größe des bewegten Fensters beträgt in der vorliegenden Arbeit $3 \times 3$ Pixel.

Contrast und Variance korrelieren in vielen praktischen Anwendungen stark miteinander. Contrast wird in vielen Studien bevorzugt eingesetzt. So verwenden Koch et al. (2002) Contrast zur Trennung verschiedener Baumarten, Yuan et al. (1991) zur Klassifikation der Vitalität von Bäumen und Clausi (2002) sowie Baraldi und Parmiggiani (1995) zur Segmentierung von Land- und Eisbedeckungsklassen.

\subsubsection{Formmerkmale}

Alle Kenngrößen der Baumkronen beziehen sich auf den im Luftbild sichtbaren Bereich der Lichtkrone. Es werden jeweils zwei Methoden A und B zur Berechnung vorgestellt, die sich für eine visuelle Auswertung mit einzelnen Punktmessungen und für eine digitale Auswertung auf der Grundlage von Rasterdaten eignen. Berechnungen von A (visuelle Auswertung) wurden mit dem Pascal-Programm KRONE (Heydecke und Fuchs 2000) durchgeführt. Berechnungen von B (digitale Auswertung) wurden mit Shell-Skripten und der Verknüpfung verschiedener Module (r.volume, r.surf.area, r.mapcalc, r.le.patch, r.transect, r.report, r.univar, r.slope.aspect) innerhalb von GRASS realisiert.

Kronenschirmfläche. A: Die Kronenschirmfläche $(K S F)$ einzelner Bäume wird gemäß der Gaußschen Dreiecksformel für geschlossene Polygonzüge aus den Lagekoordinaten der Kronenrandpunkte berechnet (Kahmen 1993).

B: In digitalen Bildern wird die Anzahl der Pixel innerhalb eines Segmentes ermittelt und mit der Fläche eines Pixels multipliziert.

Kronenlänge. A: Die Kronenlänge $(K L)$ ist die Differenz aus der Höhe der Kronenspitze $Z_{S p}$ und den gemittelten Höhen der sichtbaren Kronenrandpunkte $\bar{K}_{R}$. Sie wird aus den Z-Koordinaten der Baumspitze und der mittleren Höhe der gemessenen Kronenrandpunkte berechnet als:

$$
K L=Z_{S p}-\bar{K}_{R}
$$

B: Die Kronenlänge wird als Spannweite der normalisierten Höhen des Oberflächenmodelles innerhalb eines Kronenpolygones bestimmt.

Kronenbreite. Die Kronenbreite wird über die Gleichung

$$
K B=2 \sqrt{\frac{K S F}{\pi}}
$$

als Durchmesser eines Kreises, dessen Flächengröße der Kronenschirmfläche entspricht, hergeleitet. 
Kronenmantelfläche und Kronenvolumen. A: Sie werden für Fichten, Eichen und Buchen aus geometrischen Modellkörpern bestimmt. Eichen- und Buchenkronen werden mit einem Kugelschnitt, Fichten als Kegel approximiert (Tab. 3.9, Abb. 3.10):
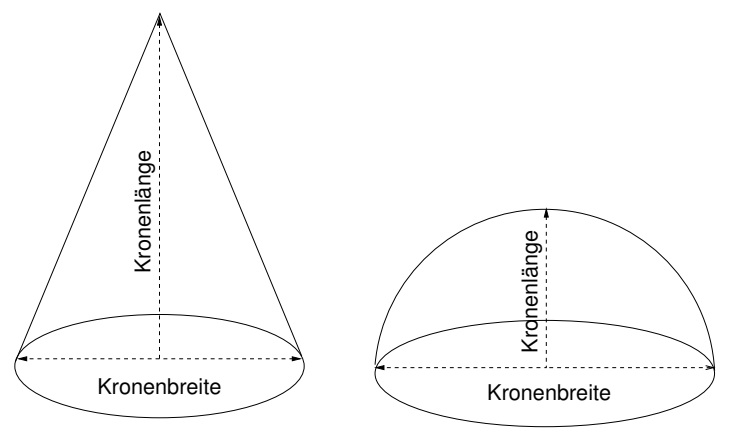

Abb. 3.10: Kegel und Kugelschnitt

Tab. 3.9: Mantelfläche und Volumen geometrischer Modellkörper

\begin{tabular}{llll}
\hline $\begin{array}{l}\text { Modell- } \\
\text { körper }\end{array}$ & $\begin{array}{l}\text { Baum- } \\
\text { art }\end{array}$ & $\begin{array}{l}\text { Kronen- } \\
\text { mantelfläche } \\
\text { (KMF) }\end{array}$ & Kronenvolumen (KVOL) \\
\hline $\begin{array}{l}\text { Kugel- } \\
\text { schnitt }\end{array}$ & $\begin{array}{l}\text { Eiche, } \\
\text { Buche }\end{array}$ & $K M F=\pi\left(\frac{K B^{2}}{4}+K L^{2}\right)$ & $K V O L=\frac{3}{\pi} K L^{2}\left(\frac{3}{2} K B-K L\right)$ \\
Kegel & Fichte & $K M F=\pi \frac{K B}{2} \sqrt{\frac{K B^{2}}{4}+K L^{2}}$ & $K V O L=\frac{1}{3} K S F \cdot K L$ \\
\hline
\end{tabular}

B: Die Kronenmantelfläche wird mit dem GRASS-Befehl r.surf.area als Summe der Dreiecksflächen von dreidimensional triangulierten Punkten (Pixelzentren) im jeweiligen Segment berechnet (GRASS Benutzerhandbuch, GDT 2002). Die Ergebnisse der Berechnung sind abhängig von der geometrischen Auflösung, die mit 0,25 m Kantenlänge der Pixel in der Bodenauflösung sehr klein gewählt wurde. Abb. 3.11 visualisiert die unregelmäßigen Kronenoberflächen von Eichen, Buchen und Fichten als Illuminations- und Drahtmodelle.

Bei Verwendung von Rasterdaten wird das Kronenvolumen $V_{k}$ mit dem GRASSBefehl r.volume ermittelt. Die Fläche jedes Pixels der Kantenlänge $s$ wird mit der Dif- 
ferenz $N D O M-\min \{N D O M\}$ multipliziert:

$$
V_{k}=s^{2} * \sum_{i}^{N_{G}}\left(N D O M_{i}-\min \left\{N D O M_{i}\right\}\right)
$$

wobei:

$$
\begin{aligned}
s & =\text { Kantenlänge der Pixel, } \\
\min \left\{\text { NDOM }_{i}\right\} & =\text { Minimum der normalisierten Höhen im Segment, } \\
i & N_{G}=\text { Anzahl der Pixel mit Grauwerten innerhalb des Segmentes, } \\
\text {NDOM }_{i} & =\text { normalisierte Höhe. }
\end{aligned}
$$

Position der Kronenspitze ( $\left.x_{s p}, y_{s p}, z_{s p}\right)$. A: Die Messmarke wird auf den höchsten aus der Krone herausragenden Ast aufgesetzt und die Koordinaten gespeichert.

B: Die Koordinaten des Maximums eines normalisierten Oberflächenmodelles innerhalb eines Segmentes $\begin{gathered}\max \left\{N D O M_{i}\right\} \\ i\end{gathered}$

Position des Baumfußes $\left(x_{t}, y_{t}, z_{t}\right)$. Die Baumfußkoordinaten der Bäume liegen nach einer tachymetrischen Vermessung im Gelände als dreidimensionale Koordinaten vor $\left(z_{t}=\right.$ terrestrisch ermittelte Höhe). Die Lagekoordinaten $x, y$ können aus den Koordinaten der Kronenspitze näherungsweise bestimmt werden. Fuchs und Cho (2001) beobachten relativ geringe mittlere Lagefehler von 2,2 bis 2,7 m als Abweichungen der visuellen fotogrammetrischen Kronenspitzen von den terrestrisch eingemessenen Baumfüßen in einem Eichen-Buchen-Mischbestand.

Position Flächenschwerpunkt $\left(x_{c p}, y_{c p}\right)$. B: Der Flächenschwerpunkt der Kronenschirmfläche als Massenschwerpunkt berechnet sich nach der Formel:

$$
x_{c p}=\frac{\sum m_{j} \cdot x_{j}}{\sum m_{j}}, y_{c p}=\frac{\sum m_{j} \cdot y_{j}}{\sum m_{j}}
$$

wobei

$x_{j}, y_{j}=$ Koordinaten der Pixel, die zu einem Segment gehören,

$m_{j} \quad=$ Masse des Pixels $j$.

Bei Rasterdaten sind die Massen $m_{j}$ gleich eins (Haberäcker 2000).

Umfang. B: Der Umfang ist die Länge eines Segmentrandes. Alle mit einem Segmentwert codierten Segmente werden daraufhin untersucht, ob die vier oder acht nächsten Nachbarn ebenfalls denselben Code besitzen. Bildpunkte, für die dieses nicht zutrifft, sind Randpixel, die aufsummiert werden. 


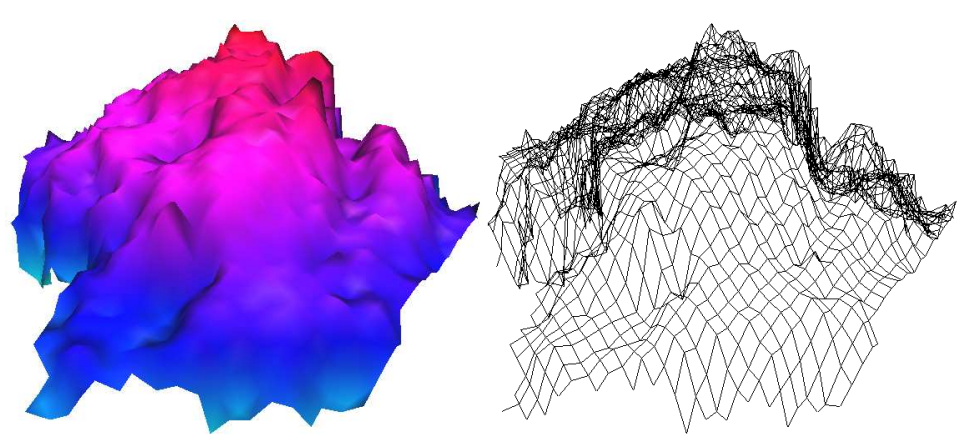

(a)

(b)

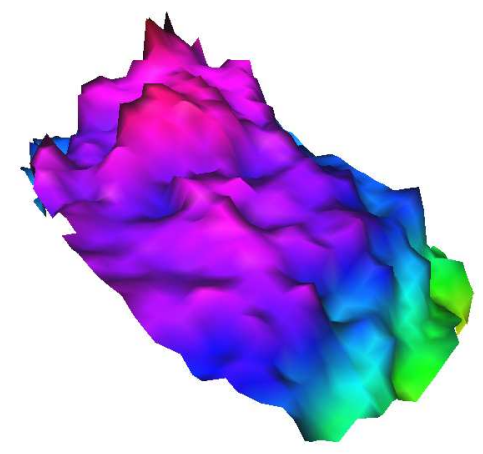

(c)

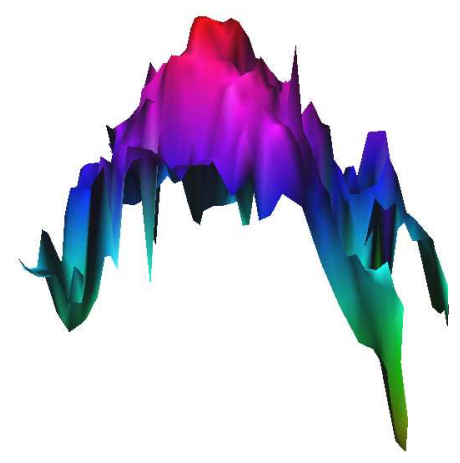

(e)

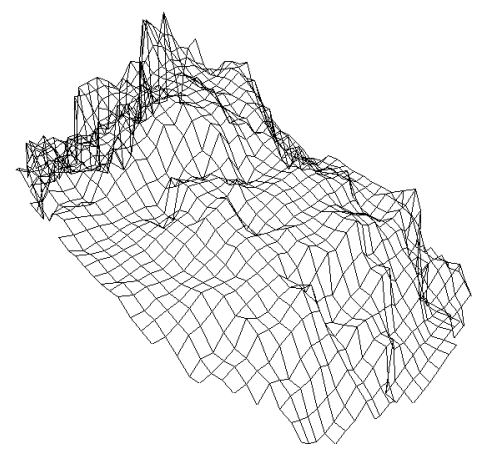

(d)

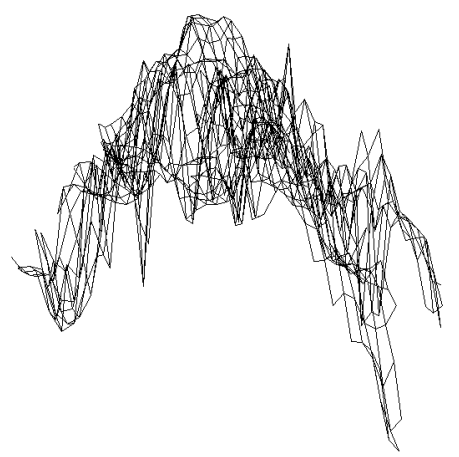

(f)

Abb. 3.11: Digitale dreidimensionale Oberflächenmodelle einer Eichenkrone $(a, b)$, Buchenkrone (c, d) und Fichtenkrone (e, f) als Illuminations- und Drahtmodelle 
Kompaktheit. Als Formindex wird häufig das einfache Verhältnis von Umfang und Fläche verwendet (z. B. Baatz und Schäpe 2000, Austin 1984). Das Verhältnis variiert mit der Flächengröße, deshalb wird dieses für die Form eines Kreises normalisiert mit

$$
K=\frac{0,282 \cdot \text { Umfang }}{\sqrt{\text { Fläche }}} .
$$

Der Index kann Werte von 1,0 für einen Kreis, 1,12 für ein Quadrat und unendlich für lange und schmale Formen annehmen und ist mit Hilfe von r.le.patch (GRASS-Modul) aus Rasterdaten einfach zu ermitteln.

Orientierung. Die Orientierung eines Segmentes wird aus der Annäherung an eine Ellipse ermittelt, deren große und kleine Hauptachse die Orientierung bestimmen (Haberäcker 2000). Die Berechnungsschritte sind:

1. Bestimmung der Koordinaten des Flächenschwerpunktes (Arc/Info Grid-Befehl: zonalcentroid)

2. Berechnung der Kovarianzmatrix, des Flächeninhaltes des Segmentes und daraus die Eigenwerte $\lambda_{1}$ und $\lambda_{2}$ und Eigenvektoren der Kovarianzmatrix. Die Orientierung wird mit dem größeren der beiden Eigenwerte $\left(\lambda_{1}\right)$ berechnet (z. B. mit dem Arc/Info Grid-Befehl zonalcentroid). Ist das Segment ein Kreis und sind beide Eigenwerte bzw. Halbachsen gleich, wird eine Orientierung von $0^{\circ}$ oder $180^{\circ}$ zugeordnet.

Länglichkeit. Das Verhältnis der Eigenwerte kann als abgeleitetes Merkmal für die Beschreibung der Länglichkeit eingesetzt werden als

$$
L=\frac{\lambda_{1}}{\lambda_{2}}
$$

Bei einem nicht länglichen Segment ist der Quotient 1, bei länglichen Segmenten deutlich größer als 1 (Haberäcker 2000).

Rauhigkeit. Ein einfaches Maß für die Rauhigkeit einer Baumkrone ist das Verhältnis der Oberflächenausdehnung einer Krone (Kronenmantelfläche) zur orthogonalen Flächengröße (Kronenschirmfläche) (Hildebrandt 1996, Wolff et al. 1999):

$$
C R=\frac{K M F}{K S F}
$$

Krümmung. Die folgenden Definitionen wurden Alexandrov et al. (1989) entnommen. Die Krümmung einer Oberfläche an einem gegebenen Punkt auf der Oberfläche kann durch das Ausmaß charakterisiert werden, mit dem die Oberfläche ihre tangentiale Ebene verläßt. In Abb. 3.12 wird die Krümmung eines gegebenen Punktes durch eine Vielzahl von Kurven definiert, die auf der Oberfläche liegen und durch den gegebenen Punkt in verschiedenen Richtungen verlaufen. Es wird eine tangentiale Ebene durch den Punkt M konstruiert und die spezifische Richtung der Flächennormalen bestimmt, die 
senkrecht auf der Tangentialebene steht und durch den Punkt M verläuft. Dann werden Flächenkurven betrachtet, die durch solche Ebenen ausgeschnitten werden, die durch die Flächennormale im Punkt M verlaufen. Diese Kurven werden Normalschnitte genannt. Nach dem Theorem von Euler kann gezeigt werden, dass in jedem Punkt einer Oberfläche zwei besondere Richtungen existieren:

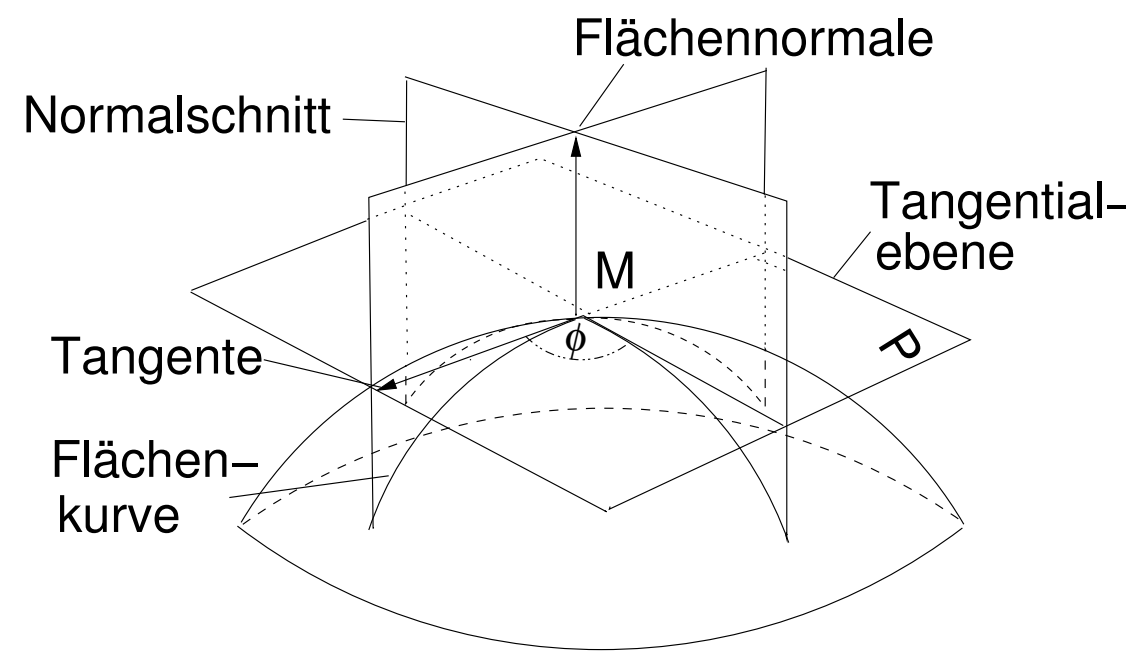

Abb. 3.12: Begriffe zur Definition der Oberflächenkrümmung eines gegebenen Punktes $\mathrm{M}$

1. diese stehen senkrecht zueinander,

2. die Krümmungen $k_{1}$ und $k_{2}$ der Normalschnitte in diesen Richtungen haben die kleinsten und größten Werte (Extremwerte) aller Normalschnitte,

die Krümmung $k(\phi)$ des Normalschnittes, der von dem Schnitt der Krümmung $k_{1}$ mit dem Winkel $\phi$ gedreht ist, wird definiert mit:

$$
k(\phi)=k_{1} \cdot \cos ^{2} \phi+k_{2} \cdot \sin ^{2} \phi .
$$

Die Krümmungen $k_{1}$ und $k_{2}$ werden Hauptkrümmungen einer Oberfläche an einem gegebenen Punkt genannt. Zu ihnen gehören die Hauptkrümmungsrichtungen, die senkrecht zueinander stehen.

Die Gaußsche Krümmung einer Oberfläche von einem gegebenen Punkt ist das Produkt der Hauptkrümmungen (minimale und maximale Krümmung):

$$
k=k_{1} * k_{2} \text {. }
$$

Das Vorzeichen der Gaußschen Krümmung definiert den Charakter der Oberfläche in der Nähe des betrachteten Punktes. Für $k>0$ ist die Oberfläche konvex ( $k_{1}$ und $k_{2}$ haben das gleiche Vorzeichen) und für $k<0$, wenn $k_{1}$ und $k_{2}$ verschiedene Vorzeichen haben, ist die Oberfläche konkav. Dreschler (1981) verwendet die Gaußsche Krümmung und 
die Hauptkrümmungen für die automatische Bildzuordnung von Punkten in digitalen Bildern.

In der vorliegenden Arbeit wird der absolute Wert der Gaußschen Krümmung berechnet, der den Grad der Krümmung einer Oberfläche im Allgemeinen angibt und als Abstraktion von verschiedenen Krümmungen in verschiedenen Richtungen verstanden werden kann (Alexandrov et al. 1989).

Das Modul r.slope.aspect in GRASS verwendet ein multiples lineares Regressionsmodell mit Wechselwirkung der Gleichung

$$
z=\beta_{0}+\beta_{1} x+\beta_{2} y+\beta_{3} x^{2}+\beta_{4} y^{2}+\beta_{5} x y+\varepsilon .
$$

Zur Näherung einer Oberfläche innerhalb kleiner Ausschnitte werden die sechs Regressionskoeffizenten mit der Methode der Kleinsten Quadrate geschätzt. Die Größe des Oberflächenausschnittes wurde durch eine Fenstergröße von $5 \times 5$ Pixel festgelegt. Die Hauptkrümmungen $k_{1}$ und $k_{2}$ können aus den Regressionskoeffizienten bestimmt werden (Wood 1996):

$$
\begin{aligned}
& k_{1}=-\beta_{3}-\beta_{4}-\sqrt{\left(\beta_{3}-\beta_{4}\right)^{2}+\beta_{5}} \\
& k_{2}=-\beta_{3}-\beta_{4}+\sqrt{\left(\beta_{3}-\beta_{4}\right)^{2}+\beta_{5}} .
\end{aligned}
$$

Kronenprofile. B: Mit dem Shell-Skript r.rad.sh wurde ein Programm erstellt, das folgende Schritte durchführt:

1. Einlesen eines normalisierten Oberflächenmodelles;

2. Bestimmung der Lage der Kronenspitze als maximaler Wert innerhalb des Kronenpolygons;

3. Die Kronenspitze ist der Ursprung eines Polarkoordinatensystemes, in dem im Abstand von $22.5^{\circ}$ sechzehn Strahlen einer bestimmten Länge berechnet werden (r.transect);

4. Lesen und Speichern der Werte des normalisierten Oberflächenmodelles entlang der Strahlen;

5. Visualisierung der Transekte mit dem Modul d.profile;

6. Gegenüberliegende Radien werden zu acht Kronenprofilen zusammengefasst (Abb. 3.13).

Die Kronenprofile können zur Analyse von Einzelbäumen verwendet werden. Abb. 3.13 visualisiert die Lichtkrone der Eiche 48010, Fläche 502. Das aufwändige Abloten der Kronen im Gelände oder die Verwendung eines Kronenfensters (Hussein et al. 2000) können entfallen. Die Profile können als Grundlage für die Entwicklung von baumartenspezifischen Modellen der Lichtkrone dienen. 

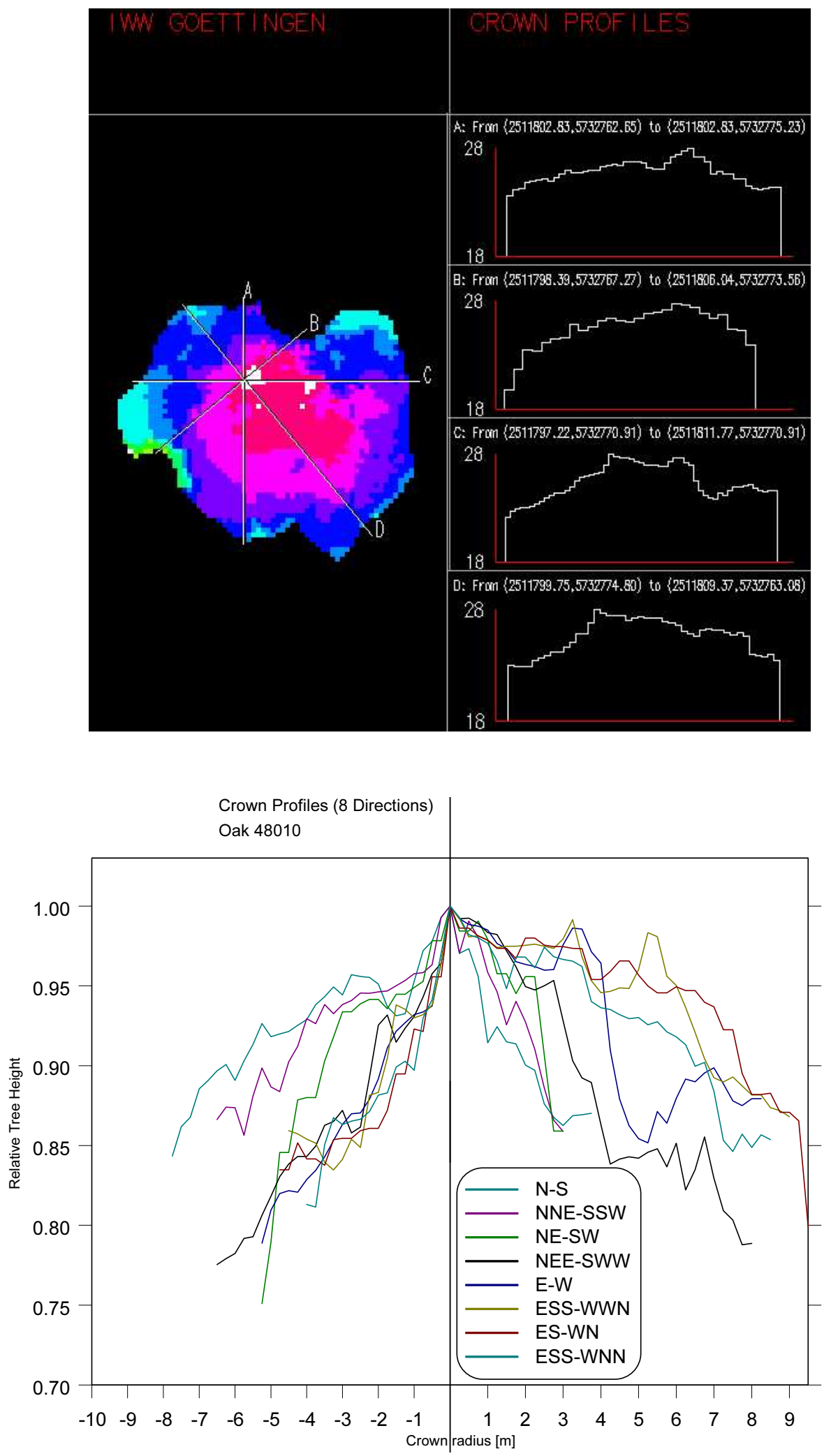

Abb. 3.13: Visualisierung der Kronenprofile der Eiche 48010, Fläche 502 


\subsubsection{Baumhöhe und Höhenänderung}

Die Luftbild-Baumhöhe (LH) ist die Differenz zwischen den Z-Koordinaten der Baumspitze $\left(z_{s p}\right)$ und des Baumfußpunktes $\left(z_{t}\right)$ :

$$
L H=z_{s p}-z_{t} .
$$

A: Die Höhe der Baumspitze wird fotogrammetrisch im Stereomodell gemessen, während die Höhe des Baumfußpunktes aus Luftbildern nur exakt erhoben werden kann, wenn der Boden in größeren Bestandeslücken sichtbar ist. In Beständen mit geringen Überschirmungsgraden wird von Akça (1989) die Messung von Geländepunkten in der Nähe der Probeflächen und die Berechnung einer Regressionsebene $z_{b}=f\left(x_{b}, y_{b}\right)$ aus den Raumkoordinaten der Bodenpunkte $\left(x_{b}, y_{b}, z_{b}\right)$ empfohlen. Mit Hilfe der Lagekoordinaten der Baumspitze $\left(x_{s p}, y_{s p}\right)$ lässt sich dann die Höhe des Baumfußpunktes $z_{t}$ aus der Regressionsebene schätzen.

Eine andere Möglichkeit ist die Erstellung eines Geländemodelles aus der terrestrischen Vermessung der Versuchsflächeninfrastruktur und der Baumfußpositionen. Zur Modellierung des Geländemodelles der Fläche 502 wurden unregelmäßig verteilte Höhenpunkte mit einem Spline-Algorithmus (GRASS-Modul s.surf.rst) interpoliert (Mitasova und Hofierka 1993).

B: Die Baumhöhe der digitalen Auswertung ergibt sich aus dem Wert des normalisierten digitalen Oberflächenmodelles an der Position der Kronenspitze.

Zur Ermittlung der Baumhöhenänderung innerhalb eines Zeitraumes ist die Berechnung eines Geländemodelles nicht notwendig. Die Höhenänderung eines Einzelbaumes wird als Differenz

$$
H Z=z_{\text {spe }}-z_{\text {spa }}
$$

der z-Koordinaten der Kronenspitze am Anfang $\left(z_{s p a}\right)$ und Ende der Messperiode $\left(z_{s p e}\right)$ bestimmt.

\subsubsection{6 Überschirmungsgrad}

Der Überschirmungsgrad ist ein Bestandesmerkmal, das effektiv in Luftbildern erhoben werden kann. Die Kronenüberschirmung ist definiert als der Flächenanteil einer Untersuchungsfläche oder einer Bestandesfläche, der von Baumkronen bedeckt ist. Er kann maximal $100 \%$ betragen:

$$
\ddot{U} \text { berschirmungsgrad }=\frac{\ddot{U} \text { berschirmte Bestandesfläche }}{\text { Gesamt fläche }} \cdot 100 \text { Prozent } .
$$

Um den Überschirmungsgrad aus Luftbildern zu bestimmen, werden häufig visuelle Schätzungen durch den Vergleich mit Kronendichteskalen durchgeführt, Stichproben mit einem Punktraster erhoben oder Kronenschirmflächen von Einzelbäumen bzw. Bestandeslücken kartiert. 
A: In dieser Arbeit wurden Kronenschirmflächen der Bäume visuell kartiert, deren Baumfuß innerhalb der Grenzen der Untersuchungsflächen liegen. Dieses Verfahren kann zu einer Über- oder Unterschätzung des Überschirmungsgrades führen. Schirmflächen von Bäumen, deren Baumfuß außerhalb der Untersuchungsfläche liegen, aber trotzdem in die Untersuchungsfläche hineinragen, werden nicht berücksichtigt. Eine methodisch folgerichtige Kartierung muss also zusätzliche Baumkronen in einem Randstreifen berücksichtigen. Dieses ist bei größeren Flächen ( 0,5 ha) mit einem beträchtlichen Mehraufwand der visuellen Auswertung verbunden. Die Kronenschirmflächen der Bäume innerhalb der Untersuchungsfläche wurden deshalb vollständig berücksichtigt, auch wenn diese über den Rand der Untersuchungsfläche hinausragten. Eine andere Möglichkeit besteht in der Definition einer Teilfläche innerhalb der Untersuchungsfläche durch Berücksichtigung eines Randstreifens, dessen Breite dem Abstand der mittleren Kronenbreite entspricht.

B: Eine überwachte Klassifikation des digitalen Orthofotos wurde als erste Stufe der digitalen Auswertung durchgeführt. Hierzu wurden sowohl spektrale Signaturen des Orthofotos als auch Höhen eines normaliserten Oberflächenmodelles eingesetzt. Die Ergebnisse einer digitalen Klassifikation können auf eine größere Fläche in der Nähe der Untersuchungsflächen übertragen werden und erlauben eine Schätzung des Überschirmungsgrades mit geringerem Aufwand als eine flächige visuelle Kartierung. Hierzu wurde ein Ausschnitt mit einer Fläche von 5,5 ha aus dem Bestand in der Nähe der Fläche 502 ausgewählt. In einer ersten Teilstudie wurden zwei Klassen definiert: Krone und Schatten-Lücke. Eine zweite Teilstudie führte zur Unterscheidung von drei Klassen: Krone-Licht, Krone-Schatten und Lücke-Schatten. Als Trainingsgebiete des überwachten Klassifikationsverfahrens wurden die fotogrammetrisch bestimmten Kronenschirmflächen für die Klasse Krone definiert. Die Klasse Krone-Schatten konnte aus der Beleuchtungs- und Sonnengeometrie zum Zeitpunkt der Bildaufnahme und dem digitalen Oberflächenmodell mit dem GRASS-Befehl r.sunmask modelliert werden. Die Klasse Lücke-Schatten wurde ausgewählt, indem zusätzliche Polygone innerhalb des Bildausschnittes digitalisiert wurden (GRASS-Modul v.digit). Da keine terrestrischen Referenzdaten vorlagen, wurde die Übereinstimmung zwischen den überwachten Klassifikationsergebnissen und einer Luftbildinterpretation anhand von Testflächen überprüft.

\subsubsection{Stehendes Totholz}

A: Totholz wird als Kronenverlichtungsstufe 4 (Blattverlust 100\%) im Rahmen der Waldzustandsinventuren erfasst. In großmaßstäbigen CIR-Luftbildern ist diese Stufe durch eine starke Veränderung der Rückstrahlung gegenüber vitaler Belaubung gekennzeichnet (totaler Blattverlust). In geschlossenen Laubholzbeständen sind häufig nur noch der Stamm und wenige Hauptäste erkennbar.

B: Zur Klassifikation des stehenden Totholzes wurde ein einfaches Schwellenwertverfahren angewendet. Der Differenzkanal $V I_{\text {diff }}=G R \ddot{U} N-R O T$ der spektralen Farbauszüge des CIR-Filmes ist gut geeignet für die Auswahl eines Schwellenwertes, mit dem alle Pixelwerte unterhalb eines bestimmten Grenzwertes ausgewählt werden kön- 
nen. Diese kennzeichnen den typischen Reflexionsgrad von kahlen Ästen mit geringen Anteilen im Infrarot und hohen Blau- und Grün-Anteilen.

Mit Hilfe der GRASS-Funktion r.clump können zusammenhängende Regionen definiert werden. Mit einem Bufferverfahren (r.buffer) werden diese mit einer Distanzzone von 2 m erweitert . Das entspricht einer Modellvorstellung einer Baumkrone mit einem minimalen Kronenradius von $2 \mathrm{~m}$. Für die so entstandenen Segmente wird ein mittlerer Vegetationsindex (NDVI) berechnet. Mit der Auswahl eines zweiten Schwellenwertes können nun abgestorbene kahle Äste innerhalb von Baumkronen von stehendem Totholz unterschieden werden. Der mittlere NDVI trennt stehendes Totholz mit überwiegenden Schattenanteilen von Ästen innerhalb lebender Bäume. In Abb. 3.14 wird das nach dieser Methode klassifizierte Totholz wiedergegeben. Ein vergrößerter Ausschnitt des Orthofotos 1988, überlagert mit den Vektoren der Totholzsegmente, veranschaulicht stehendes Totholz und abgestorbene Äste von Bäumen mit starkem Blattverlust.

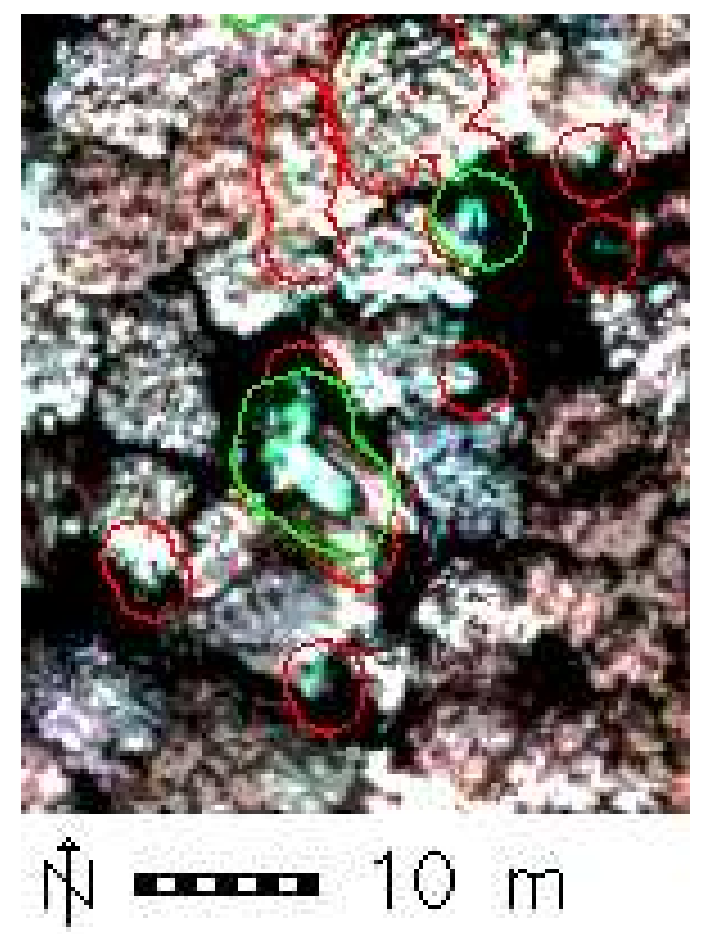

Abb. 3.14: Ausschnitt aus einem digitalen Farbinfrarot-Orthofoto mit abgestorbenen Bäumen (stehendes Totholz, grüne Vektoren) und abgestorbenen Ästen innerhalb lebender Baumkronen (rote Vektoren) als Ergebnis eines Schwellenwertverfahrens

\subsubsection{Erkennung von ausgeschiedenen Bäumen}

Die Erfassung von Bäumen, die durch Holzeinschlag entnommen werden, ist für die Überwachung von Bewirtschaftungsmaßnahmen als auch für die Veränderung der Bestandesstruktur von Bedeutung. 
A: Visuelle Bildauswertungen können mit der Segmentierung der Baumkronen der jüngsten Bildaufnahme oder der mittleren Aufnahme beginnen. Bei hohen Überschirmungsgraden des Bestandes ist eine Vektorisierung der Kronendachlücken effektiver (vgl. Münch 1995). Durch Überlagerung der Vektoren der Kronen- bzw. Lückenpolygone über jüngere bzw. ältere digitale Bildaufnahmen werden Veränderungen deutlich.

B: Die Veränderungen des Kronendaches können in einer Zeitreihe von drei Aufnahmezeitpunkten mit der Berechnung des normalisierten Differenz-Vegetationsindex (NDVI) und der Darstellung des RGB-Farbsystems auf dem Bildschirm visualisiert werden (Sader und Winne 1992). Der NDVI wurde aus den digitalen Orthofotos von 3 verschiedenen Befliegungsjahren 1993, 1994 und 1995 berechnet und in eine 8 bitAuflösung transformiert. Grüne Vegetation absorbiert proportional mehr elektromagnetische Strahlung im roten Spektralbereich und weniger im nahinfraroten Spektralbereich. Hohe Anteile grüner Biomasse sind deshalb mit hohen NDVI-Werten, geringe Anteile grüner Biomasse mit niedrigen NDVI-Werten verbunden.

Die Kenntnis des Aufnahmezeitpunktes und die Zuordnung zu einer der additiven Grundfarben Blau, Rot und Grün erlaubt eine Erfassung der Änderungen im Kronendach. Die Gesetze der additiven Farbmischung sind Grundlage für die Interpretation der multitemporalen Farbkomposite. Wenn zwei primäre Farben mit gleichen Helligkeiten vorliegen, lassen sich hieraus die Komplementärfarben blaugrün, purpur und gelb mischen. Kombinationen von allen drei primären Farben Blau, Rot und Grün gleicher Helligkeit ergeben Weiß, die Abwesenheit der drei Grundfarben erzeugt schwarz. Wenn ein Baum in einem bestimmten Jahr ausscheidet, so verringert sich die Reflexion im nahinfraroten und roten Spektralbereich und damit der NDVI-Wert, dem eine entsprechende additive Grundfarbe zugeordnet ist. Die Farbe, die in der Farbkomposite entsteht, kann visuell interpretiert werden. Mit einem RGB-Cluster-Verfahren können die ursprünglichen $256^{3}$ Farben auf 27 Farben reduziert werden (GRASS Funktion r.composite). Die Klassenanzahl kann weiter reduziert werden mittels einer visuellen Zusammenfassung der Klassen. Im vorliegenden Beispiel wurden drei der 27 Klassen zusammengeführt, und ein neues Binärbild erstellt. Zusammenhängende Pixel bekommen ein einheitliches Attribut (GRASS-Function r.clump). Anschließend werden alle Flächen kleiner als eine definierte Mindestgröße (hier $6 \mathrm{~m}^{2}$ ) eliminiert (GRASS-Funktion r.reclass.area).

\subsubsection{Ablauf der Luftbildauswertung}

Es werden zwei Auswertungsverfahren vorgestellt, die als visuelle und digitale Auswertung bezeichnet werden. Sie unterscheiden sich hinsichtlich des rechnergestützten Anteiles an der Informationsverarbeitung. Bei der visuellen Auswertung ${ }^{3}$ hängt der überwiegende Teil der Informationsverarbeitung von den Augen und dem Gehirn eines Auswerters ab. Die technischen Arbeiten werden an einem analytischen Stereoplotter oder aber auch an einem digitalen fotogrammetrischen System (DFS) mit einem Bild-

\footnotetext{
${ }^{3}$ Im englischen Sprachgebrauch wird diese auch - wenig treffend - als „,manual interpretation“ bezeichnet.
} 
schirm für Stereosicht durchgeführt. Die digitale Auswertung ist gekennzeichnet durch den überwiegenden Anteil eines Computers an der Informationserfassung.

\subsubsection{Visuelles Auswertungsverfahren}

Die Luftbilder aller Befliegungsjahre wurden auf allen Untersuchungsflächen mit dem visuellen Verfahren ausgewertet. Dieses Verfahren entspricht dem Standard der Option 4 und 5 der höchsten Kategorie des Handbuches der europäischen Arbeitsgruppe „Remote Sensing“(WGRSA 2000).

Die Arbeitsschritte des Verfahrens werden in der folgenden Reihenfolge unter der Annahme ausgeführt, dass analoge Luftbilder und terrestrische Referenzdaten, Passpunkte bzw. topographische Karten vorliegen:

1. Wenn ein DFS verwendet wird, ist das Digitalisieren der analogen Luftbilder notwendige Voraussetzung - dies entfällt beim analytischen Stereoplotter,

2. Bildorientierung (s. Kapitel 3.3.2.2),

3. Stereosicht - nach abgeschlossener Orientierung kann mit der eigentlichen stereoskopischen Auswertung begonnen werden,

4. Lokalisierung der Untersuchungsfläche und Kartierung der Grenzen,

5. Messen von 5-8 Geländepunkten in der Nähe der Untersuchungsfläche, falls kein externes Geländemodell vorliegt,

6. Zuordnung der Baumkronen zu terrestrisch eingemessenen Baumfußpositionen oder Kronenkarten, wenn diese zur Verfügung stehen,

7. Baumartenerkennung,

8. Messen der Positionen der Kronenrandpunkte,

9. Messen der Kronenspitze,

10. Erstellung einer Kronenkarte, Überprüfung und Anpassung des Interpretationsschlüssels im Gelände,

11. Vergleich der Merkmale des Interpretationschlüssels mit den terrestrischen Kronenansprachen und den Bildinhalten vor Beginn der visuellen Luftbildinterpretation,

12. Interpretation des Vitalitätszustandes nach Blatt- / Nadelverlust in $5 \%$-Stufen, Chlorosestufen in $5 \%$-Stufen, soziale Stellung, Baumklassen nach Kraft in den Stufen 1 - 4,

13. Auswertung und Kontrolle der Interpretationsergebnisse. 


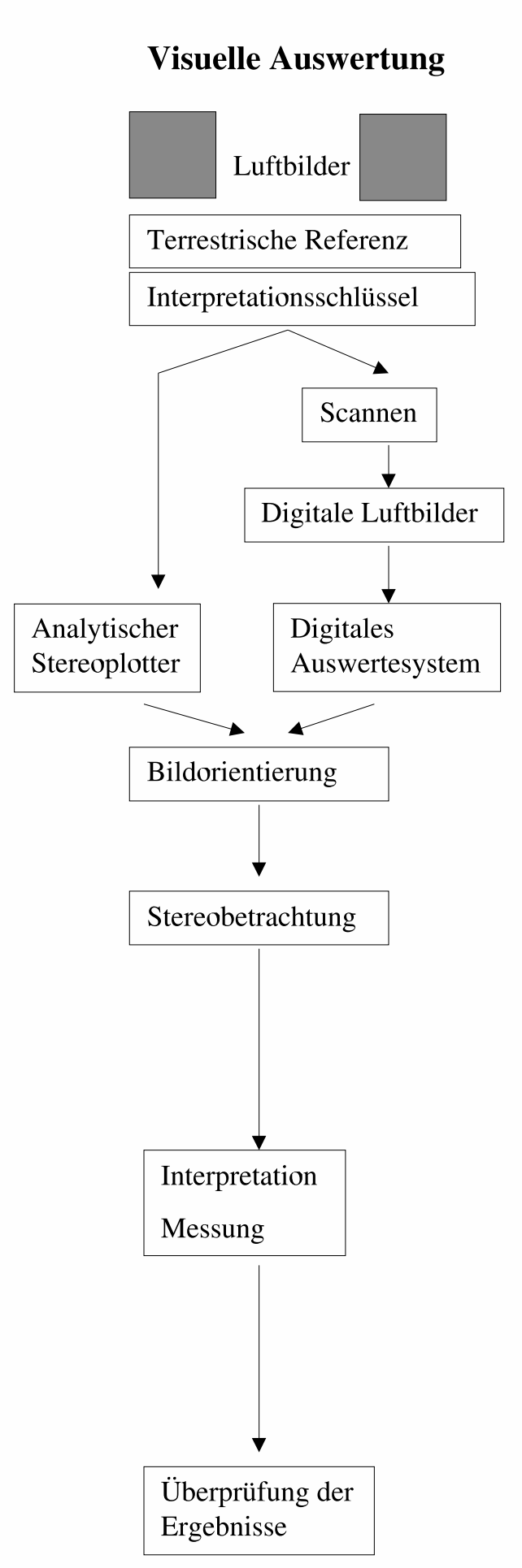

\section{Digitale Auswertung}

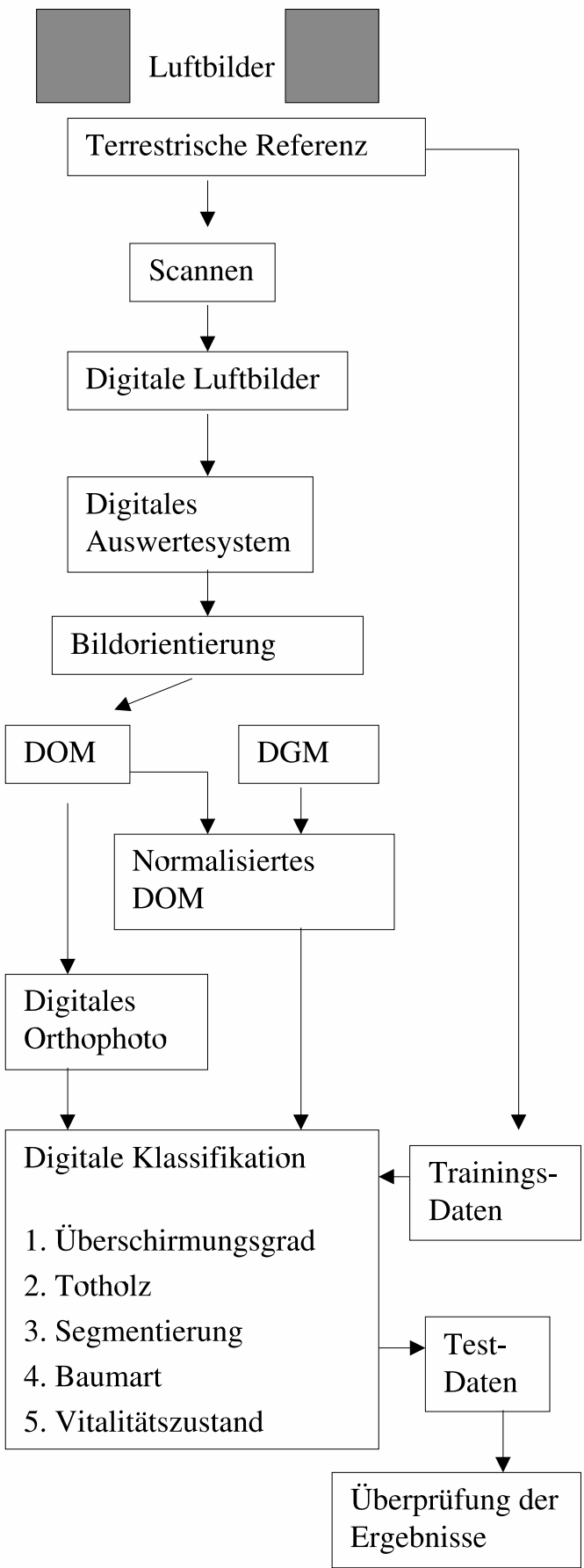

Abb. 3.15: Arbeitsschritte der visuellen und digitalen Luftbildauswertung 


\subsubsection{Digitales Auswertungsverfahren}

Zur digitalen Auswertung werden digitale Bilder und ein digitales fotogrammetrisches System vorausgesetzt. Nach abgeschlossener Bildorientierung wird ein digitales Oberflächenmodell (DOM) mit einer geringen Schrittweite von 0,25 bis 0,5 m mit Hilfe automatischer Bildzuordnung erstellt. Dieses ist Grundlage für die Herstellung eines digitalen Orthofotos mit einer räumlichen Auflösung von 0,25 m. Ein Geländemodell wird aus einer visuellen Stereoauswertung der Luftbilder oder aus externen Datenquellen erzeugt. Im nächsten Schritt wird ein normalisiertes Oberflächenmodell (NDOM) aus der Differenz von DOM und DGM abgeleitet.

Das digitale Orthofoto und das normalisierte Oberflächenmodell sind Grundlage eines hierarchischen Vorgehens (Abb. 3.15) zur thematischen Klassifikation. In der ersten Stufe wird das digitale Bild in den Vorder- und Hintergrund zerlegt, d. h. in Baumkronen und Schatten bzw. Bestandeslücken. Diese Entscheidung kann mit einem ML(Maximum-Likelihood-) oder SMAP- (Sequential-Maximum-A-Posteriori-) Klassifikator getroffen werden. Die Objektklasse Totholz wird mit einem einfachen Schwellenwertverfahren unter Verwendung eines Differenzkanales aus dem grünen und roten Spektralbereich identifiziert. Im nächsten Schritt werden einzelne Kronen abgegrenzt. Eine visuelle stereoskopische Delinierung des Kronenrandes ist auf kleinen Untersuchungsflächen durchführbar und bisher das optimale Verfahren.

In einer Teilstudie werden Baumkronen mit einem Wasserscheidenalgorithmus unter Verwendung des normalisierten Oberflächenmodelles segmentiert. Die SegmentMittelwerte der spektralen und räumlichen Merkmale werden zur Trennung der Baumarten eingesetzt. Als Klassifikationsverfahren wird eine lineare Diskriminanzanalyse angewendet. In der letzten Hierarchiestufe werden mit dem gleichen Verfahren drei Vitalitätsstufen nach dem Blattverlust getrennt.

\subsubsection{Genauigkeit digitaler Höhenmodelle}

Während die Genauigkeit der Höhenmessung mittels digitaler Fotogrammetrie für die Erstellung von Geländemodellen auf Gletschern, in offenen Landschaften und Stadtgebieten untersucht wurde, gibt es nur wenige systematische Untersuchungen zur vertikalen Genauigkeit der digitalen fotogrammetrischen Systeme in Waldgebieten (z. B. Adler 2001, Sysavath et al. 2001). Wälder werden als problematische Bereiche ausgewiesen und aus Genauigkeitsuntersuchungen ausgeklammert (Baltsavias und Käser 1998), als „Geländerauschen“ (Heipke 1995) oder ,3-D-Störung“ (Gülch 1994) bezeichnet. Kraus (1997, S. 227) gibt Definitionsunsicherheiten bei der fotogrammetrischen Punktmessung von Sträuchern und Bäumen an, die im Bereich von 20 bis $100 \mathrm{~cm}$ liegen. Die terrestrische Bestimmung von Waldoberflächen ist aufwendig oder unmöglich. Die Genauigkeit der digitalen Höhenmodelle wurde deshalb mit Hilfe verschiedener Vergleichsmessungen untersucht.

Als Referenz wurden Höhenmessungen aus einer visuellen Messung mit dem analytischen Stereoplotter DSR 18 eingesetzt. Die visuelle Zweibildauswertung mit analytischen Auswertegeräten ist bisher das Verfahren mit der höchsten erreichbaren Messge- 
nauigkeit in Luftbildern. Theoretische und empirische Genauigkeitsschätzungen gehen von 0.06 - 0.08\%o der Flughöhe aus (Kraus 1997, S. 226). Konecny und Lehmann (1984, S. 54) leiten die Höhengenauigkeit der fotogrammetrischen Messung als Standardabweichung $\sigma_{z}$ her:

$$
\sigma_{z}=\frac{h_{g}}{c} \cdot \frac{h_{g}}{b} \cdot \sigma_{p x}
$$

wobei

$$
\begin{aligned}
h_{g} & =\text { Flughöhe über Grund } \\
b & =\text { Aufnahmebasis im Bild } \\
c & =\text { Kammerkonstante } \\
\sigma_{p x} & =\text { Standardabweichung der x-Parallaxenmessung }
\end{aligned}
$$

Die Höhengenauigkeit eines Punktes ist demnach abhängig vom Basisverhältnis $b v=$ $\frac{b}{h_{g}}$, vom Bildmaßstab $M_{b}=\frac{c}{h_{g}}$ und der x-Parallaxen-Genauigkeit $\sigma_{p x}$. Konecny und Lehmann (1984) nehmen für Weitwinkelaufnahmen $\sigma_{z}=0,065 \%$ o bei kontrastarmen Punkten $\sigma_{z}=0,1 \%$ (vgl. Jacobsen 2002, S. 17) an.

Zum anderen wurden terrestrische Baumhöhen als Referenz verwendet. Auf der Fläche 502 standen trigonometrische Messungen an stehenden Bäumen (Eiche und Buche) aus den Jahren 1995 (LÖBF) und 2000 (eigene Aufnahme) zur Verfügung.

Wiederholungen der Erstellung eines digitalen Oberflächenmodelles mit verschiedenen digitalen fotogrammetrischen Systemen, Berechnungsstrategien, Bildkanälen und geometrischer Scanner-Auflösung sollten Hinweise auf eine Verbesserung der Präzision geben.

Den Versuchsaufbau zur Überprüfung der Höhengenauigkeit digitaler fotogrammetrischer Auswertesysteme gibt das Flußdiagramm Abb. 3.16 wieder. Der Maßstab der Luftbilder und die Wahl der Scannerauflösung haben direkte Auswirkungen auf die Wirtschaftlichkeit (Speicherplatz, Rechenzeiten) und Qualität einer digitalen fotogrammetrischen Auswertung (vgl. Kraus 1996, S. 296 ff.). Die originalen Luftbilder Nr. 56 und 57 der Fläche 502 Kleve, Befliegungsjahr 1999, Maßstab 1: 3500 wurden mit dem fotogrammetrischen Scanner Wehrli RM2 (Scanner A) und Flachbett-Scanner Mustek Paragon 1200 (Scanner B) mit verschiedenen geometrischen Auflösungen digitalisiert. Aus den digitalisierten Originalluftbildern mit den Kanälen NIR, Rot und Grün wurde in einem weiteren Verarbeitungsschritt die erste Hauptkomponente (PCA1) als zusätzlicher Bildkanal berechnet.

Die analogen Luftbilder wurden mit einer einstufigen Bündelausgleichung des analytischen Stereoplotters DSR 18 orientiert. Die äußeren Orientierungsparameter (Drehwinkel: Längsneigung $\varpi$, Querneigung $\phi$ und Kantung $\kappa$; Koordinaten des Projektionszentrums: X, Y, Z ; jeweilige Standardabweichungen) wurden exportiert und in die drei DFS „SoftPlotter“, „OrthoBase“ und „OrthoEngine“ eingelesen (s. Abb. 3.16). Die Verwendung der Bildorientierung aus dem Stereoplotter sollte die Orientierungsfehler konstant halten und somit gleiche Voraussetzungen für den Vergleich der Genauigkeit 


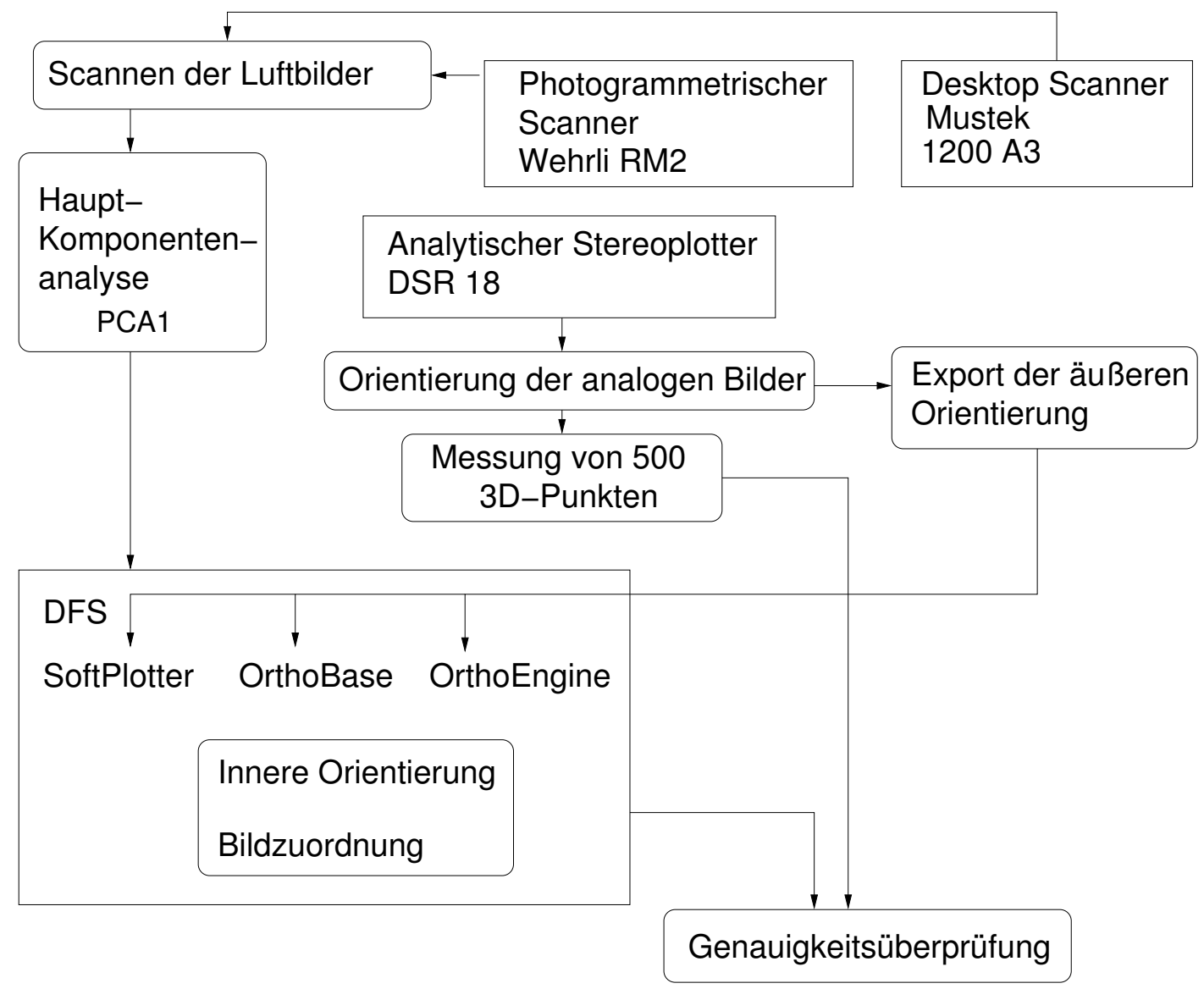

Abb. 3.16: Flußdiagramm zum Vergleich der automatischen Höhenermittlung digitaler fotogrammetrischer Systeme mit Messungen am analytischen Stereoplotter

der Höhenmodelle der DFS bieten. Es wurden 500 Punkte als systematische Stichprobe eines Luftbildmodelles mit einer Schrittweite von 26,7 m bestimmt. Unter Verwendung des analytischen Stereoplotters DSR 18 wurde jeder Stichprobenpunkt visuell interpretiert und einer Landbedeckungsklasse zugeordnet (s. Tab. 4.3).

Die Schrittweite zur Berechnung der digitalen Höhenmodelle wurde mit 0,2 m für die Digitalisierintervalle 12 - $48 \mu \mathrm{m}$ und 0,4 m für $96 \mu \mathrm{m}$ sehr klein gewählt, um Fehler einer anschließenden Interpolation möglichst gering zu halten. Bei der Auswahl der Steuerungsparameter für die Berechnung der Oberflächenmodelle wurde auf Standardeinstellungen der Software-Hersteller zurückgegriffen. Im SoftPlotter wurden zwei verschiedene Strategien (mit und ohne Interpolation bzw. Filterung (Postprocessing)) ausgewählt.

In einem weiteren Experiment wurden Messwiederholungen im Überdeckungsbereich von drei aufeinanderfolgenden Luftbildern durchgeführt. Bei einer Längsüberdeckung von $80 \%$ entsteht ein Bereich, der für die Bildzuordnung in drei verschiedenen Stereomodellen geeignet ist. 307 Punkte der Bildpaare (56/57 = Modell Rechts, 57/58 
$=$ Modell Mitte, 56/58 = Modell Links) wurden hierzu ausgewählt. Die äußere Orientierung der drei Luftbilder erfolgte unter Verwendung von signalisierten Passpunkten mit einer einstufigen Bündelausgleichung des DFS SoftPlotter.

\subsubsection{Statistische Auswertung}

\subsubsection{Geometrische Genauigkeit}

Die Genauigkeit eines raumbezogenen Datensatzes ist definiert als die Nähe der Ergebnisse von Beobachtungen, Berechnungen und Schätzungen zu den wahren Werten oder den als wahr angenommenen Werten. Es wird zwischen geometrischer und thematischer Genauigkeit unterschieden.

Tab. 3.10: Statistische Kennwerte zur Höhengenauigkeit

\begin{tabular}{|c|c|c|}
\hline Beschreibung & Symbol & Formel \\
\hline $\begin{array}{l}\text { Differenz für jedes } \\
\text { Meßwertpaar } i\end{array}$ & $d_{i}$ & $d_{i}=z_{i}-w_{i}$ \\
\hline Mittlere Differenz & $\bar{d}$ & $\bar{d}=\frac{\overline{i=1}}{n}$ \\
\hline $\begin{array}{l}\text { Mittlere absolute } \\
\text { Differenz }\end{array}$ & $d_{a}$ & $d_{a}=\frac{i=1}{n}$ \\
\hline $\begin{array}{l}\text { RMS-Fehler oder } \\
\text { mittlerer Fehler }\end{array}$ & $R M S E$ & $R M S E=\sqrt{\frac{\sum\left(z_{i}-w_{i}\right)^{2}}{n}}$ \\
\hline Median & median & median $=\left\{\begin{array}{cc}d_{\left(\frac{n+1}{2}\right)} & \text { wennn ungerade } \\
\frac{d_{\left(\frac{n}{2}\right)}+d\left(\frac{n}{2}+1\right)}{2} & \text { wennngerade }\end{array}\right.$ \\
\hline $\begin{array}{l}\text { Median der } \\
\text { absoluten Differenz }\end{array}$ & $M A D$ & $M A D=1,483 \cdot$ median $\left|d_{j}-\operatorname{median}\left(d_{i}\right)\right|$ \\
\hline $\begin{array}{l}\text { Schwellenwert für } \\
\text { Extremwerte }\end{array}$ & $N_{\max , \min }$ & $N_{\max , \min }=\operatorname{median}\left(x_{i}\right) \pm 3 \cdot M A D$ \\
\hline
\end{tabular}

wobei

$$
\begin{aligned}
n & =\text { Anzahl der Messpaare } \\
z & =\text { Höhe im digitalen Oberflächenmodell } \\
w & =\text { Referenzhöhe mit höherer Genauigkeit gemessen }
\end{aligned}
$$


Um verschiedene Methoden der Oberflächenerstellung zu vergleichen, wird die mittlere quadratische Abweichung (mean square error, MSE) verwendet (Millard und Nagaraj 2001, Mendenhall et al. 1990). Die MSE einer Schätzung ist die mittlere quadratische Abweichung vom zu schätzenden Parameter. Es kann gezeigt werden, dass die MSE gleich der Varianz plus dem Quadrat des Bias (Verzerrung) ist. Der root mean square error (RMSE oder „mittlerer Fehler“) ist die Wurzel aus der MSE und wird bevorzugt, da die Einheiten der Variablen beibehalten werden (FGDC 1998).

Im vorliegenden Versuchsaufbau wurde Höhen einer systematischen Punktstichprobe aus einem Luftbildmodell erhoben. Die wahren Höhenwerte der Population sind nicht bekannt. Deshalb werden die Fehler aus Vergleichsmessungen anderer Beobachtungsmethoden geschätzt.

Als Schwellenwert für die Ausscheidung von groben Fehlern (Extremwerten) wird der Median und der Median der absoluten Differenz (MAD) berechnet (Gülch 1994) (s.

Tab 3.10).

\subsubsection{Thematische Genauigkeit}

Als Kriterien zur Überprüfung der Übereinstimmung von zwei Klassifikationen derselben Objekte wurde die Gesamtgenauigkeit und das Kappa-Maß (Cohen 1960) berechnet. Die Kappa-Statistik wird als ein geeigneteres Maß betrachtet als die summarische Gesamtgenauigkeit. Sie gibt die Verbesserung der Übereinstimmung im Vergleich zu einer zufälligen Übereinstimmung an und kann für nominale als auch für ordinale Daten verwendet werden.

Zur Berechnung wird eine quadratische Kontingenztabelle oder Fehlermatrix angefertigt. Jedes Objekt wird nach Maßgabe seiner Klassenzugehörigkeit als Klassifikationsergebnis oder Referenz ${ }^{4}$ eingetragen (Tab. 3.11):

Tab. 3.11: Aufbau einer Fehlermatrix für eine Klassifikation mit $k$ Klassen

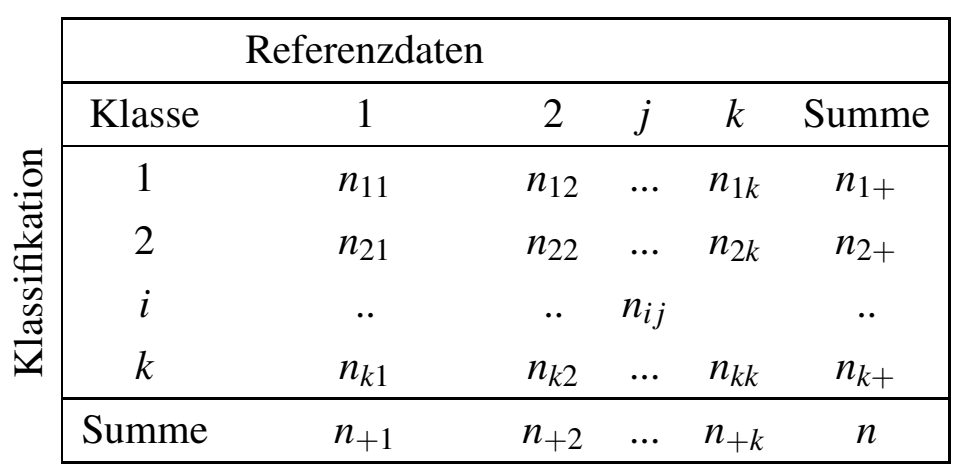

\footnotetext{
${ }^{4}$ bzw. als erste oder zweite Beobachtung bei Wiederholungen
} 
Als einfaches beschreibendes Maß wird die Gesamtgenauigkeit als Summe der Objekte in der Diagonalen dividiert durch die Gesamtzahl der Beobachtungen definiert als:

$$
P_{0}=\frac{\sum_{i=1}^{k} n_{i i}}{n}
$$

Die erwartete Übereinstimmung $P_{e}$ ist die Summe der Kreuzprodukte der beobachteten Anteile in Reihen und Spalten und gibt den Anteil der zufällig korrekt klassifizierten Objekte wieder

$$
P_{e}=\frac{\sum_{i=1}^{k} n_{i+} n_{+i}}{n^{2}}
$$

wobei

$n \quad=$ Anzahl der Beobachtungen in der Stichprobe

$n_{i i}=$ Anzahl der Beobachtungen in der Klasse $i$ für die Klassifikation und Klasse $i$ für die Referenz

$n_{i+}=$ Anzahl aller Beobachtungen in der Klasse $i$ für die Klassifikation

$n_{+i}=$ Anzahl aller Beobachtungen in der Klasse $i$ für die Referenz.

Das Kappa-Maß $\kappa$ berechnet das Verhältnis zwischen der Verbesserung der beobachteten Gesamtgenauigkeit zur zufälligen Übereinstimmung und der theoretisch höchst möglichen Verbesserung zur zufälligen Übereinstimmung:

$$
\kappa=\frac{P_{0}-P_{e}}{1-P_{e}} .
$$

Ein Kappa-Maß von 1 zeigt eine perfekte Übereinstimmung zwischen Beobachtungen an, ein Wert von 0 bedeutet, dass die Übereinstimmung derjenigen entspricht, die auch bei einer Zufallsklassifikation erreicht worden wäre. Dieses würde bedeuten, dass mit dem angewendeten Klassifikationsverfahren keine Steigerung der Genauigkeit erzielt wird gegenüber einer uneingeschränkten Zufallsauswahl. Bei negativen Werten ist die Übereinstimmung geringer als bei einer zufälligen Klassifikation. Das Kappa-Maß ist immer kleiner als oder gleich der Gesamtgenauigkeit.

Als zusätzliche Kennwerte können der Standardfehler des Kappa-Maßes und eine Test-Statistik angegeben werden. Die Test-Statistik der R-Funktion „kappaFor2“ (Baron und Li 2001) basiert auf einem zweiseitigen Z-Test mit einem P-Wert für die NullHypothese $\kappa=0$.

\subsubsection{Klassifikation}

Das Problem einer Klassifikation besteht darin, die Gruppen- / Klassenzugehörigkeit von Pixeln oder Bildsegmenten aus den Merkmalswerten zu schätzen. Bei der überwachten Klassifikation sind die ordinal oder nominal skalierten Gruppen vorgegeben. Datensätze, für die die Gruppenzugehörigkeit mit anderen Mitteln festgestellt wurde 
(Feldaufnahmen oder Luftbildinterpretation), dienen dazu, die Verteilungen der Merkmalswerte zu schätzen. Diese werden Trainings-Daten genannt. Der Bildauswerter hat durch die Auswahl von Trainings-Daten großen Einfluß auf das Ergebnis der Klassifikation.

Es wurden drei verschiedene überwachte Klassifikationsalgorithmen für eine quantitative Analyse des digitalen Orthofotos und Oberflächenmodelles verwendet: der Maximum-Likelihood-Algorithmus (ML), der Sequential-Maximum-A-Posteriori-Algorithmus (SMAP) von Bouman und Shapiro (1994) und die lineare Diskriminanzanalyse für metrisch skalierte Variablen. Die Algorithmen von ML und SMAP sind in GRASS implementiert. Die lineare Diskriminanzanalyse wurde mit der Bibliothek „MASS“ in dem Statistik-System R als Funktion lda und lda.predict durchgeführt (Venables und Ripley 2000).

ML ist einer der am häufigsten verwendeten Algorithmen in der Auswertung multispektraler Fernerkundungsdaten. Die Gruppenzuordnung von einzelnen Pixeln wird nach der maximalen Wahrscheinlichkeit getroffen, die aus Trainings-Daten geschätzt wird. Die Trennungsfunktion erfordert die Berechnung der Kovarianzen und Mittelwertvektoren. Dabei wird eine Normalverteilung der Merkmalswerte vorausgesetzt ( $\mathrm{Ri}$ chards und Xiuping 1999).

Der SMAP-Algorithmus berücksichtigt nicht nur einzelne Pixel, sondern gleichzeitig die räumliche Umgebung in verschiedenen Auflösungen. Der räumliche Kontext wird durch ein Markov'sches Zufallsfeld modelliert. Die Aneinanderreihung von Zufallsfeldern in einer Pyramidenstruktur mit verschiedenen Auflösungen von grob bis fein wird Markov-Kette genannt. Die Wahrscheinlichkeiten des höher aufgelösten Zufallsfeldes ist abhängig von den Wahrscheinlichkeiten des vorherigen geringer aufgelösten $\mathrm{Zu}$ fallsfeldes. Als Trennfunktion stellen Bouman und Shapiro (1994) einen "SequentialMaximum-A-Posteriori“ Schätzer (SMAP) vor, der den räumlichen Fehler minimiert.

Die Diskriminanzanalyse ist eine Methode zur Überprüfung von Gruppenunterschieden. Sie ermöglicht eine gleichzeitige Untersuchung der Bedeutung mehrerer abhängiger Variablen für die Unterscheidung der Gruppen. Ein weiterer Anwendungsbereich besteht in der Klassifikation neuer Objekte, wobei vorausgesetzt wird, dass Datensätze mit bekannter Gruppenzugehörigkeit vorliegen.

Ausgehend von einer vorgegebenen Gruppierung eines Trainings-Datensatzes werden Koordinatenachsen berechnet, die eine optimale Trennung der Gruppen ermöglichen. Die Kriterien für die Verschiedenheit der Gruppen sind zum einen der Abstand zwischen den Gruppenmittelpunkten und zum anderen die Variation innerhalb der Gruppen. Die Zielsetzung besteht nun in der Konstruktion einer neuen Achse, auf der das Abstandsquadrat zwischen den Gruppenmittelpunkten möglichst groß und die Summe der quadrierten Abweichungen vom jeweiligen Gruppenmittelpunkte möglichst klein ist. Diese Rotationstransformation der abhängigen Merkmalsvariablen läßt sich als Linearkombination der Messwerte darstellen, d. h. das Auffinden der optimalen Position der neuen Achse ist gleichbedeutend mit der Festlegung von Gewichtskoeffizienten für die Variablen, so dass eine optimale Gruppentrennung erreicht wird. Der theoretische Hintergrund und Rechenschritte werden ausführlich in Bortz (1999) sowie Bahrenberg etal. (1992) erläutert. Die lineare Diskriminanzanalyse wurde als über- 
wachtes Klassifikationsverfahren für die Trennung der Baumarten Buche, Eiche und Vitalitätsstufen nach Blattverlust angewendet.

Die Eignung der Variablen für eine Klassentrennung wurde mit einem Auswahlverfahren nach Smith (beschrieben in Huberty 1994) beurteilt. Die Auswahl der besten Variablen ist bei korrelierenden Variablen problematisch, da die Bedeutung einer Variablen davon abhängt, welche anderen bereits ausgewählt wurden. Es wird deshalb ein iteratives Auswahlverfahren (schrittweise vorwärts) durchgeführt, das optimale Teilmengen der Variablen vergleicht (Huberty 1994):

1. Berechnen von $p$ univariaten Diskriminanzanalysen und Registrieren der Gesamtgenauigkeit und Kappa-Statistik für jeden Schätzer. Die beste Teilmenge der Größe 1 besteht aus derjenigen Variablen V1 mit der höchsten Trefferquote.

2. Durchführung von $p-1$ bivariaten Diskriminanzanalysen, in denen die Variablen V1 und V2, V1 und V3, V1 und Vp verwendet werden. Die Gesamtgenauigkeit und Kappa-Statistik wird für jedes Paar registriert und die beste Variablenteilmenge der Größe 2 (z. B. V1,V2) wird ausgewählt.

3. Durchführung von $p-2$ trivariaten Diskriminanzanalysen mit den Variablen V1, V2 und V3; V1, V2, und V4;..; V1, V2, und Vp, Registrieren der Genauigkeitsmaße und Auswählen des besten Triplets (z. B. V1, V2, V3) mit der höchsten Genauigkeit.

4. Dieses wird wiederholt mit allen Teilmengen der Größe $p-1$. Insgesamt werden mindestens $p(p+1) / 2$ Analysen durchgeführt.

\subsubsection{Lineare Regressionsanalyse}

Die Beziehungen zwischen den im Luftbild gemessenen Kronenvariablen (Kronenbreite, -länge, -schirmfläche, -mantelfläche und -volumen) und dem terrestrisch hergeleiteten Grundflächenzuwachs wurden mit einfachen linearen Regressionsmodellen

$$
y=\beta_{0}+\beta_{1} x+\varepsilon
$$

und multiplen linearen Regressionsmodellen

$$
y=\beta_{0}+\beta_{1} x_{1}+\beta_{2} x_{2}+\cdots+\beta_{k} x_{k}+\varepsilon
$$

untersucht. Der Grundflächenzuwachs ist die Zielgröße $y$ (,,response“ oder abhängige Variable), die Kronendimensionen $K B, K L, K S F, K M F, K V O L$ sind die Ausgangsgrößen $\mathrm{x}$ (Regressoren oder unabhängige Variablen). Beim einfachen linearen Modell ist $\beta_{0}$ der Achsenabschnitt und $\beta_{1}$ die Steigung der allgemeinen Geradengleichung. $\varepsilon$ ist der Term für die als zufällig aufgefaßte Abweichung der beobachteten Werte von der Geraden. Die Regressionskoeffizienen $\beta_{0}$ und $\beta_{j}, j=1,2, \ldots, k$, werden mit der Schätzmethode der Kleinsten Quadrate bestimmt. Im vorliegenden Fall der Untersuchung von wenigen Versuchsflächen wird davon ausgegangen, dass diese Flächen selbst schon die Grundgesamtheit darstellen. Die berechneten Regressionskoeffizienten sind daher als para- 
metrische Koeffizienten zu interpretieren; d. h., dass statistische Tests nicht angezeigt sind. Es bedeutet auch, dass die Ergebnisse streng genommen als Fallstudienergebnisse zu interpretieren sind, die sich ausschließlich auf die betrachtete Grundgesamtheit beziehen. Extrapolationen und Rückschlüsse über diese Grundgesamtheit hinaus sind mit Vorsicht zu interpretieren.

Als Maß für die Güte eines Regressionsmodelles wird das Bestimmtheitsmaß $R^{2}$ angegeben. In der einfachen linearen Regression ist das die quadrierte ProduktmomentKorrelation $r_{x y}$, die die Stärke des Zusammenhanges zwischen x und y misst. Das Bestimmtheitsmaß $R_{p}^{2}$ eines Regressionsmodelles mit $p$ Termen, d. h. $p-1$ Regressoren und einem Achsenabschnitt $\beta_{0}$, berechnen Montgomery et al. (2001) als

$$
R_{p}^{2}=\frac{S S_{R}(p)}{S S_{T}}=1-\frac{S S_{R e s}(p)}{S S_{T}}
$$

wobei $S S_{R}(p)$ die Summe der Abweichungsquadrate der Regression, $S S_{\text {Res }}(p)$ die Summe der Quadrate der Residuen oder zufälligen Abweichungen für ein Modell mit $p$ Regressionskoeffizienten und $S S_{T}$ die Gesamt-Varianz bezeichnen. Eine möglichst geringe Residualstreuung der Regression ist ein weiteres Kriterium für die Auswahl des besten Modelles:

$$
M S_{\text {Res }}(p)=\frac{S S_{\text {Res }}}{n-p}
$$

Für die univariaten Regressionsmodelle wird zusätzlich der Standardfehler der Steigung $s e_{\beta_{1}}$ angegeben, der einen Hinweis gibt, ob die Steigung $\beta_{1}$ ungleich Null ist und somit ein linearer Zusammenhang zwischen $\mathrm{y}$ und $\mathrm{x}$ gegeben ist. 


\section{Ergebnisse}

\subsection{Bewertung der automatischen Erstellung von Höhenmodellen}

\subsubsection{Vergleich mit terrestrischen Baumhöhen}

Die terrestrischen Einzelbaumhöhen, die als Referenz zu den fotogrammetrisch ermittelten Baumhöhen verwendet wurden, haben eine relativ geringe Spannweite (Tab. 4.1). Es sind Bäume der Baumklassen 1 bis 3, die im Luftbild in der Bestandesoberschicht eindeutig identifiziert werden konnten.

Tab. 4.1: Statistische Kennzahlen der Baumhöhenmessung im Wald

\begin{tabular}{cccccccc}
\hline Zeitpunkt & $N_{\text {gesamt }}$ & $N_{\text {Eiche }}$ & $N_{\text {Buche }}$ & Mean & Min & Max & Stdev \\
\hline November 1995 & 26 & 21 & 5 & 28,1 & 25,7 & 32,2 & 1,7 \\
Juli 2000 & 17 & 12 & 5 & 30,0 & 23,0 & 33,9 & 2,9 \\
\hline
\end{tabular}

Zur Bestimmung der fotogrammetrischen Baumhöhe sind jeweils zwei Messungen notwendig: die Höhe des Baumfußes und die der Kronenspitze. Nach dem Fehlerfortpflanzungsgesetz addieren sich die Fehler beider Messungen. Die höchste mittlere Abweichung zeigt das Verfahren der visuellen Kartierung (DFS SoftPlotter) unter Verwendung einer fotogrammetrischen Regressionsebene zur Herleitung der Höhe des Baumfußes. Bodenpunkte zur Bestimmung der Regressionsebene konnten aufgrund des hohen Überschirmungsgrades nur entfernt von den Untersuchungsflächen im Luftbild gemessen werden. Sämtliche Verfahren mit Verwendung des Geländemodelles aus terrestrischer Vermessung der Baumfußpunkte erzielen deshalb geringere Abweichungen (Tab. 4.2).

Der Vergleich der fotogrammetrischen mit den terrestrischen Baumhöhen gibt Hinweise auf die systematische Unterschätzung der Baumhöhen im Luftbild. Die mittleren Differenzen sind mit Ausnahme des automatischen Verfahrens 1995 negativ (Tab. 4.2). 
Tab. 4.2: Genauigkeit der fotogrammetrischen Baumhöhen von Eichen und Buchen im Vergleich zu terrestrischen Baumhöhen (Referenz)

\begin{tabular}{|c|lc|cccccccc|}
\hline Jahr & \multicolumn{2}{|c|}{ Messung } & \multicolumn{6}{|c|}{ Differenzen } \\
\hline 1995 & Krone & DGM & N & RMSE & Mean & Min & Max & Stdev & $\begin{array}{l}\text { Abs. } \\
\text { Mean }\end{array}$ \\
\hline & $\begin{array}{l}\text { DSR } \\
\text { visuell }\end{array}$ & terrestr. & 26 & 1,84 & $-0,79$ & $-4,51$ & 2,72 & 1,66 & 1,36 \\
& $\begin{array}{l}\text { Softplotter } \\
\text { visuell }\end{array}$ & fotogr. & 26 & 2,56 & $-2,07$ & $-5,00$ & 1,80 & 1,50 & 2,29 \\
& $\begin{array}{l}\text { Softplotter } \\
\text { visuell }\end{array}$ & terrestr. & 26 & 1,66 & $-0,82$ & $-4,21$ & 2,87 & 1,45 & 1,29 \\
\hline \hline 1999 & $\begin{array}{l}\text { Softplotter } \\
\text { digital }\end{array}$ & terrestr. & 26 & 1,80 & 0,13 & $-2,57$ & 5,96 & 1,79 & 1,30 \\
& $\begin{array}{l}\text { DSR } \\
\text { visuell }\end{array}$ & terrestr. & 17 & 2,36 & $-0,96$ & $-5,78$ & 2,31 & 2,16 & 1,86 \\
& $\begin{array}{l}\text { Softplotter } \\
\text { visuell }\end{array}$ & fotogr. & 17 & 4,10 & $-2,99$ & $-7,80$ & 4,20 & 2,80 & 3,72 \\
\hline & $\begin{array}{l}\text { Softplotter } \\
\text { visuell }\end{array}$ & terrestr. & 17 & 3,32 & $-1,83$ & $-6,79$ & 5,36 & 2,77 & 2,80 \\
\hline & $\begin{array}{l}\text { SoftPlotter } \\
\text { digital }\end{array}$ & terrestr. & 17 & 2,99 & $-2,33$ & $-6,97$ & 0,43 & 1,87 & 2,38 \\
\hline
\end{tabular}

DSR = analytischer Stereoplotter, SoftPlotter $=$ Digitales Fotogrammetrisches System, visuell $=$ visuelle Luftbildmessung, digital $=$ Baumhöhe aus normalisiertem digitalen Oberflächenmodell, terrestr. = DGM aus terrestrisch gemessenen Baumfußpositionen, fotogr. $=$ fotogrammetrisch gemessene Regressionsebene nach Akça (1989).

In anderen Untersuchungen wird ebenfalls über systematisch negative Abweichungen der Baumhöhen im Luftbild im Vergleich zu terrestrischen Baumhöhen berichtet. Wolff (1992) stellt eine mittlere Differenz von -0,4 m und einen RMS-Fehler von 2,6 $\mathrm{m}$ bei Höhenmessungen an einem analytischen Stereoplotter (107 Eichen) im Bildmaßstab 1:12500 fest. Bei Verwendung eines fotogrammetrischen Kronenoberflächenmodelles eines DFS berichten Kätsch und Stöcker (2000) über niedrigere Luftbildmittelhöhen von Fichten im Vergleich zu terrestrischen Baumhöhen. Nach Oester (1991) ergeben Messungen mit analytischen Auswertungsgeräten in großmaßstäbigen Luftbildern (291 Bäume) um 9\% niedrigere Höhen und eine Standardabweichung des Residuums von 2,3 m. Hoffmann (2001) ermittelt ebenfalls geringere Luftbildhöhen am DFS Softplotter im Vergleich zur terrestrischen Baumhöhe, eine mittlere absolute Differenz von $1 \mathrm{~m}$ und eine Standardabweichung von 0,92 m. Neuere Untersuchungen von Heurich et al. (2003) zur Genauigkeit von Baumhöhenmessungen aus Laserscannerdaten im Vergleich zu trigonometrischen Baumhöhen im Bayerischen Nationalpark (308 Laubbäume) stellen mittlere Differenzen von -0,37 m, eine Standardabweichung 
von 1,43 m, und eine mittlere absolute Differenz von 1,01 m fest. Eine systematische Unterschätzung der Nadelbaumhöhen aus Laserscannerdaten wird auch von anderen Autoren berichtet (Magnussen und Boudewyn 1998, Naesset 1997).

Die Ergebnisse des Vergleiches der Baumhöhenmessung im Luftbild mit der trigononmetrischen Höhenmessung im Gelände bestätigt für 1995 den Fehlerrahmen von 1 bis $2,5 \mathrm{~m}$, der in anderen empirischen Untersuchungen für überwiegend kleinere Bildmaßstäbe angegeben wird (z. B. Kätsch und Stöcker 2000, Stellingwerf und Hussin 1997, Wolff 1992, Akça et al. 1971). Bei den untersuchten Luftbildern der Befliegungen 1995 und 1999 führen geringe Basisverhältnisse $b v^{1}(0,15$ bzw. 0,12) und hohe Längsüberdeckungen $p \%^{2}$ (80 bzw. $83 \%$ ) zu eingeschränkten Höhengenauigkeiten. Höhen lassen sich bei einer sechzigprozentigen Längsüberdeckung mit größerer Bildbasis und größerem Konvergenzwinkel besser erfassen.

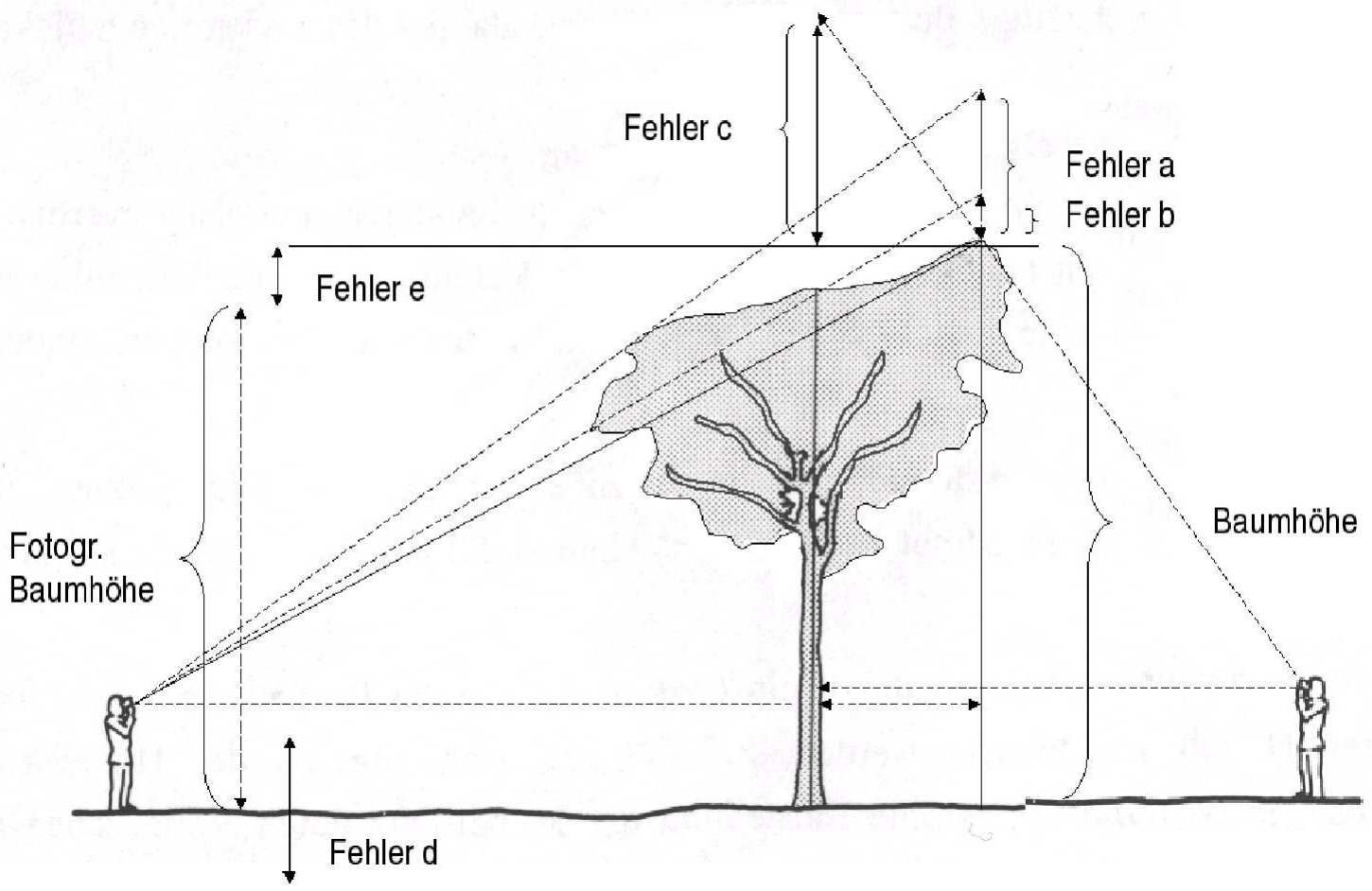

Abb. 4.1: Fehler der trigonometrischen Höhenmessung (a: Anvisieren des Kronenrandes, b: fehlerhaftes Anvisieren der Kronenspitze, c: fehlerhafte Entfernungsmessung) und Fehler der Luftbildmessung (e: begrenztes Auflösungsvermögen des Sensorsystemes, d: Fehler der Messung der Geländeoberfläche bzw. des Geländemodelles)

Für den Bildjahrgang 1999 treten jedoch größere Abweichungen auf, die auf andere Fehlerursachen zurückzuführen sind. Stellingwerf und Hussin (1997) und Spurr (1960)

\footnotetext{
${ }^{1} b v=\frac{b}{h_{g}}$, wobei $b=$ Bildbasis und $h_{g}=$ Flughöhe über Grund

${ }^{2} p \%=\frac{s-b}{s} \cdot 100$, wobei $s=$ Seitenlänge des Luftbildes und $b=$ Bildbasis
} 
nennen das begrenzte Auflösungsvermögen des Sensorsystemes als bedeutende Fehlerquelle der Luftbildhöhenmessung. Einzelne Äste oder Kronenspitzen sind häufig nicht erkennbar. Weitere Einflußfaktoren, die zu unscharfen Bildern führen, sind Bewegungen des Flugzeuges und der Bäume unter Windeinfluß.

Da die Höhenmessungen im Gelände ebenfalls beträchtliche Messfehler aufweisen können, kann die Genauigkeit der Luftbildmessung nicht absolut bestimmt werden. Die höheren Abweichungen der Baumhöhen des Bildjahrganges 1999 sind vermutlich auf den Zeitpunkt der terrestrischen Messung in der Vegetationszeit 2000 und den hohen Überschirmungsgrad zurückzuführen. Durch schlechte Sichtverhältnisse in geschlossenen Beständen und das Anvisieren des Kronenrandes der Laubbäume entstehen besonders gravierende, nicht lineare Messfehler. Die terrestrischen Baumhöhenmessungen in Laubholzbeständen sind überwiegend mit einem positiven Fehler behaftet (Pretzsch 2002, Prodan 1965). Zusätzliche Fehler entstehen durch fehlerhafte horizontale Entfernungsmessungen bei abgewölbten Kronenformen wie im untersuchten Altholzbestand der Fläche 502. Abb. 4.1 stellt die möglichen systematischen Fehler der Baumhöhenmessung im Luftbild und im Gelände am Beispiel eines Laubbaumes mit asymmetrischer Position der Kronenspitze dar.

\subsubsection{Vergleich mit Höhen aus einem analytischen Stereoplotter}

Eine systematische Stichprobe visueller Höhenmessungen wurden vom Autor am analytischen Stereoplotter DSR 18 durchgeführt. Die Mittelwerte aus drei Wiederholungsmessungen im Stereomodell 56/57, Befliegungsjahr 1999, wurden als Referenz zu den Ergebnissen der automatischen Höhenmodelle gesetzt. Die Höhen der Referenzdaten sind nicht normalverteilt, dagegen nähern sich die Differenzen der Wiederholungsmessungen einer Gaußschen Verteilung (Abb. 4.2).

Tab. 4.3: Statistische Kennzahlen der visuellen Höhenmessungen am analytischen Stereoplotter: $M_{\text {mean }}=\frac{M_{1}+M_{2}+M_{3}}{3}$

\begin{tabular}{lccccc}
\hline & $\mathrm{n}$ & $M_{\text {Mean }}$ & Min & Max & Stdev \\
\hline Gesamt & 500 & 42,15 & 26,75 & 61,81 & 9,18 \\
1 Krone-Licht & 219 & 49,8 & 32,66 & 61,81 & 5,57 \\
2 Krone-Schatten & 53 & 46,42 & 29,60 & 58,53 & 5,45 \\
3 Lücke-Boden & 4 & 29,16 & 28,19 & 29,80 & 0,71 \\
4 Lücke-Schatten & 18 & 39,66 & 26,75 & 52,74 & 8,95 \\
5 Kultur & 45 & 34,70 & 32,60 & 44,02 & 1,80 \\
6 Acker, Grünland & 144 & 32,66 & 27,53 & 46,00 & 2,34 \\
7 Bebauung & 17 & 34,65 & 30,14 & 42,16 & 2,50 \\
\hline
\end{tabular}




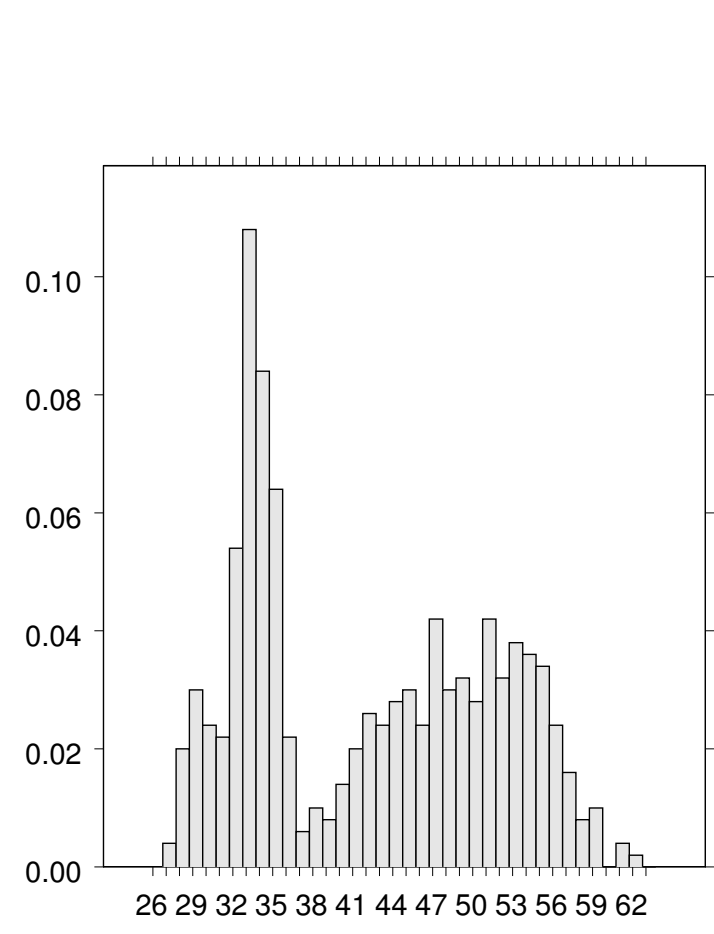

(a) Höhe ü. NN (Visuelle Messungen DSR 18)

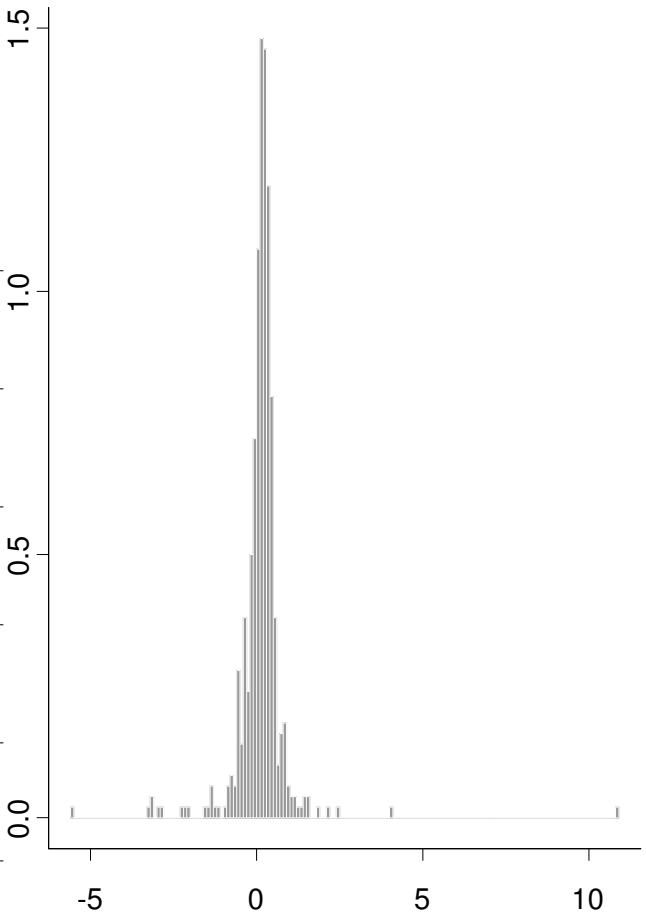

(b) Höhendifferenzen aus Wiederholungsmessungen (M2-M3, DSR 18)

Abb. 4.2: Histogramme der mittleren Höhen und Höhendifferenzen aus visuellen Wiederholungsmessungen $M_{1}-M_{2}$ (Stereoplotter DSR 18) im Stereomodell Bildnr. 56/57, 1999

Tab. 4.3 gibt einen Überblick über die Landbedeckungsklassen der 500 Testpunkte im fotogrammetrischen Modell 56/57, 1999. Der Waldanteil mit den Klassen Krone, Lücke und Laubholzkultur überwiegt vor den Klassen Acker / Grünland und Bebauung. Die Standardabweichungen der aus drei Wiederholungsmessungen gemittelten Höhenwerte sind in den Baumkronen und Lücken mit Schatten am höchsten.

Die geringsten Standardabweichungen der Differenzen zwischen visuellen Wiederholungsmessungen am analytischen Stereoplotter sind erwartungsgemäß in den Freilandklassen 6 (Acker, Grünland) und 7 (Bebauung, Verkehrsflächen) zu finden. Der Fehler der Wiederholungsmessungen ist aber auch in Bestandeslücken mit Bodensicht (Boden-Lücke) gering und liegt unter 0,5 m. Die Baumkronen im Licht werden dagegen mit höheren Standardabweichungen von 0,6 bis 1,2 m erfasst. Münch (1995) berichtet von einer Standardabweichung von 0,4 m bei Doppelmessungen der Kronenspitzen von Laubbäumen mit einem analytischen Stereoplotter. In den Klassen Kronen-Schatten, Lücke-Schatten und Laubholzkultur treten die höchsten Standarabweichungen auf (Tab. 4.4). 
Tab. 4.4: Standardabweichungen der Differenzen zwischen Wiederholungsmessungen $\left(M_{1}, M_{2}, M_{3}\right)$ am analytischen Stereoplotter

\begin{tabular}{l|c|c|c|c}
\hline \multicolumn{4}{c}{ Differenzen } \\
\hline & & $M_{1}-M_{2}$ & $M_{2}-M_{3}$ & $M_{1}-M_{3}$ \\
\hline & $\mathrm{n}$ & Stdev & Stdev & Stdev \\
Gesamt & 500 & 1,58 & 0.80 & 1.66 \\
1 Krone-Licht & 219 & 1,15 & 0,60 & 0,96 \\
2 Krone-Schatten & 53 & 1,98 & 1,77 & 3,12 \\
3 Lücke-Boden & 4 & 0,46 & 0,22 & 0,30 \\
4 Lücke-Schatten & 18 & 3,84 & 1,90 & 3,38 \\
5 Kultur & 45 & 3,16 & 0,28 & 3,15 \\
6 Acker, Grünland & 144 & 0,36 & 0,17 & 0,34 \\
7 Bebauung & 17 & 0,46 & 0,11 & 0,48 \\
\hline
\end{tabular}

In Abb. 4.3 werden die Fehlerkennwerte mit Hilfe dreier digitaler fotogrammetrischer Systeme automatisch erstellten Höhenmodellen mit visuellen Höhenwerten des analytischen Stereoplotters verglichen. Die RMS-Fehler schwanken unter Berücksichtigung aller 500 Vergleichspunkte zwischen 2,6 und 4,3 m. Die gemittelten Abweichungsbeträge und RMS-Fehler geben die geringsten Werte für das DFS SoftPlotter wieder.

Die mittlere Differenz ist bei allen digitalen fotogrammetrischen Systemen positiv. Die automatischen Höhenwerte werden im Vergleich zu den visuellen Messungen am analytischen Stereoplotter geringfügig überschätzt (Abb. 4.3).

Ausreißerwerte treten besonders zahlreich beim DFS OrthoEngine auf. Bis zu 48 $\%$ der Höhenwerte liegen außerhalb des Bereiches Median $\pm 3 \times M A D$ (Median der absoluten Differenz) (Abb. 4.3). Die Visualisierung der Ausreißer kann ein interaktives Editieren der Höhenmodelle erleichtern.

Die höchste Zahl an Ausfällen der automatischen Höhenwerte sind bei OrthoEngine anzutreffen. Der Grund liegt vermutlich in der Definition eines Schwellenwertes für die Bildzuordnung innerhalb des Algorithmus, der vom Anwender nicht beeinflußt werden kann. In einem weiteren Bearbeitungsschritt können die Bildpunkte ohne Ergebnis aus den benachbarten Höhenwerten interpoliert werden. Die Bildzuordnung vom SoftPlotter liefert im Randbereich des vorgegebenen Modellbereiches keine Ergebnisse. Durch Interpolation und Filterung der Ergebnisse ohne Höhenwerte wird die Gesamtgenauigkeit von OrthoBase gesteigert (Abb. 4.3).

Das Auswahlkriterium des Scannertyps und des Digitalisierungsintervalles beeinflusst die Höhengenauigkeit (s. Abb. 4.4). Die geringsten mittleren Fehler wurden nicht mit dem kleinsten Intervall von $12 \mu \mathrm{m}$ sondern mit $48 \mu \mathrm{m}$ am fotogrammetrischen Scan- 

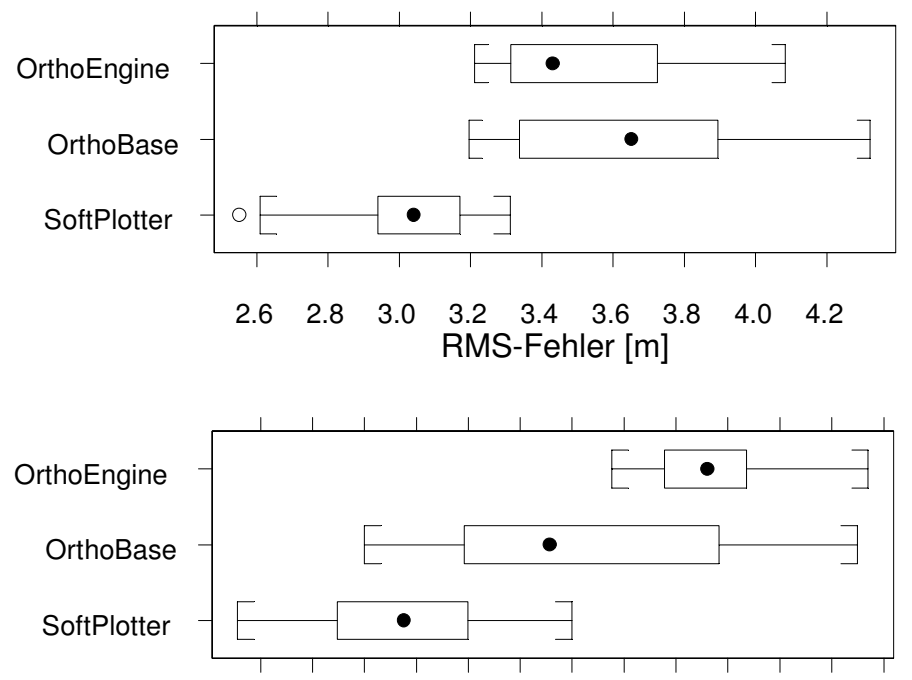

$\begin{array}{lllllllllllll}1.1 & 1.2 & 1.3 & 1.4 & 1.5 & 1.6 & 1.7 & 1.8 & 1.9 & 2.0 & 2.1 & 2.2 & 2.3\end{array}$ Mittlere absolute Differenz [m]

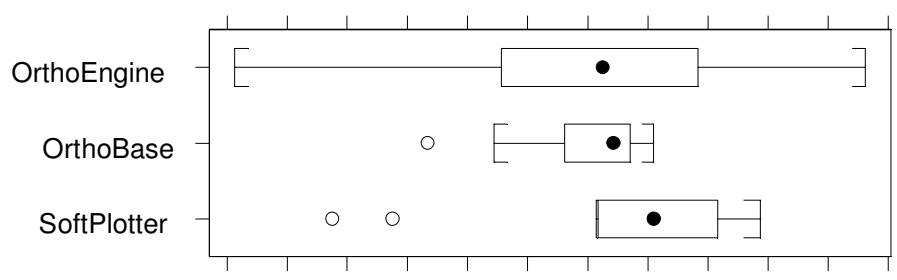

$\begin{array}{llllllllllll}0.0 & 0.1 & 0.2 & 0.3 & 0.4 & 0.5 & 0.6 & 0.7 & 0.8 & 0.9 & 1.0 & 1.1\end{array}$ Mittlere Differenz [m]
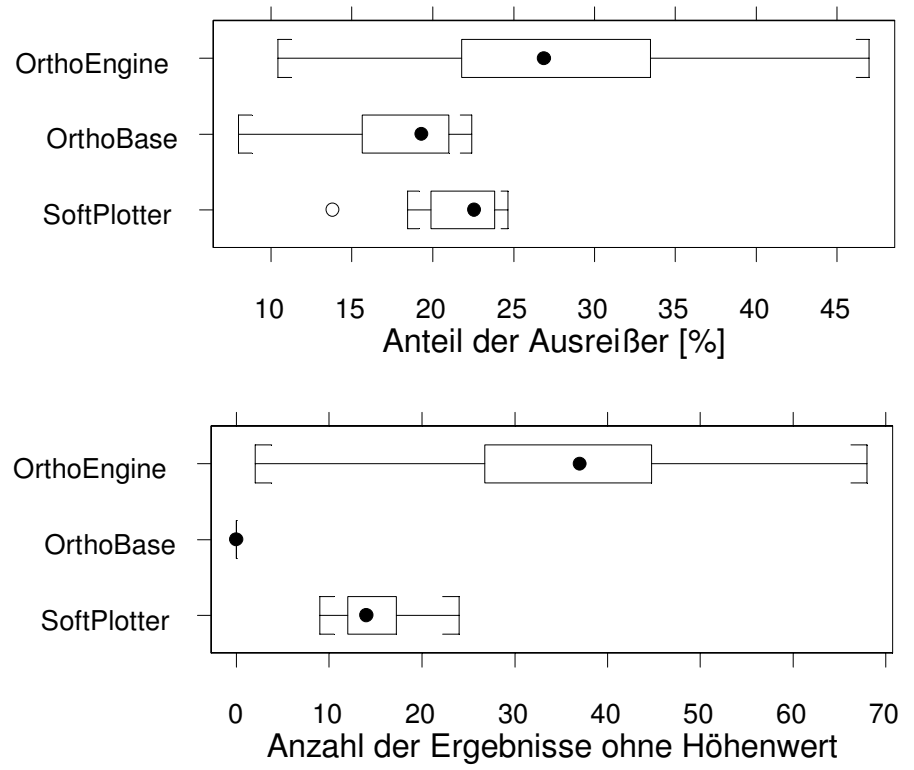

Abb. 4.3: Fehlerkennwerte der automatisch generierten Höhen minus Referenzdaten (visuelle Höhenmessungen des analytischen Stereoplotters), 500 Vergleichspunkte, 10 Wiederholungen. 
ner A erreicht. Der Büroflachbettscanner B erreicht mit einem Digitalisierungsintervall von $42 \mu \mathrm{m}$ (entspricht $600 \mathrm{dpi}$ ) unter Verwendung der Bildzuordnung mit dem DFS Softplotter relativ gute Genauigkeiten und liegt damit noch vor den Ergebnissen des Scanners A mit einem Intervall von $96 \mu \mathrm{m}$. Eine Scannerauflösung von $48 \mu \mathrm{m}$ ist für das vorliegende großmaßstäbige Bildmaterial optimal. Die höhere Detailerkennbarkeit einer geometrischen Auflösung von 12 oder $24 \mu \mathrm{m}$ rechtfertigt nicht den erheblich höheren Speicherbedarf und die längeren Rechenzeiten.

Die Auswahl der Bildkanäle NIR, Rot, Grün und der ersten Hauptkomponente (PCA1) beeinflusst die Genauigkeit eines automatischen Höhenmodelles, was für drei DFS untersucht wurde. Die Verwendung der ersten Hauptkomponente ergibt eine geringe Steigerung der Genauigkeit gegenüber der Verwendung des infraroten Kanals bei SoftPlotter und OrthoEngine (Abb. 4.4).

RMS-Fehler Mittlere absolute Differenz

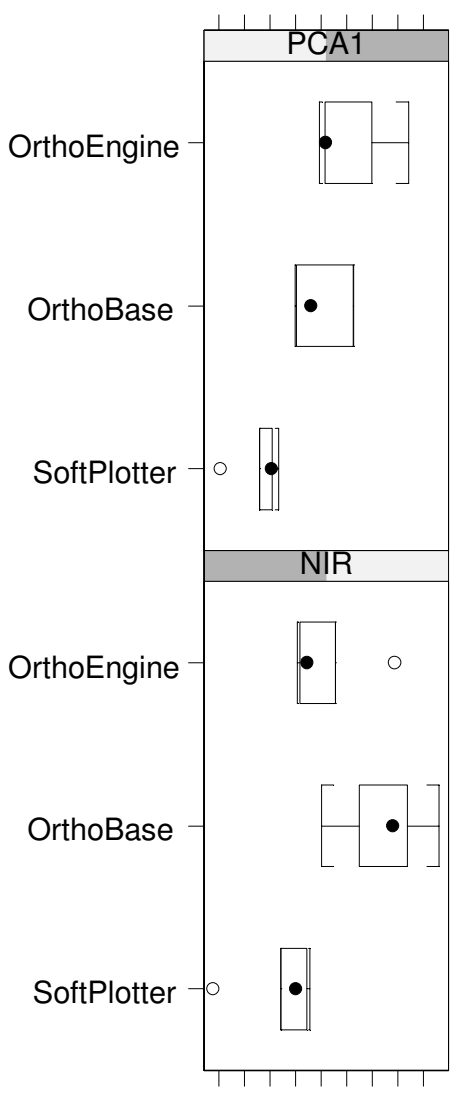

2.63 .03 .43 .84 .2

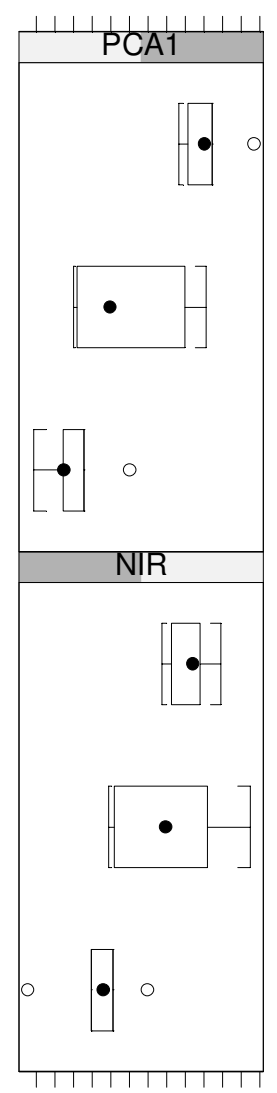

$\begin{array}{llll}1.1 & 1.4 & 1.7 & 2.0 \\ {[\mathrm{~m}]} & 2.3\end{array}$

Abb. 4.4: Vergleich der automatisch generierten Höhen minus Referenzdaten (visuelle Höhenmessungen des analytischen Stereoplotters) in Abhängigkeit vom DFS und Bildkanal 
RMS-Fehler

Mittlere absolute Differenz

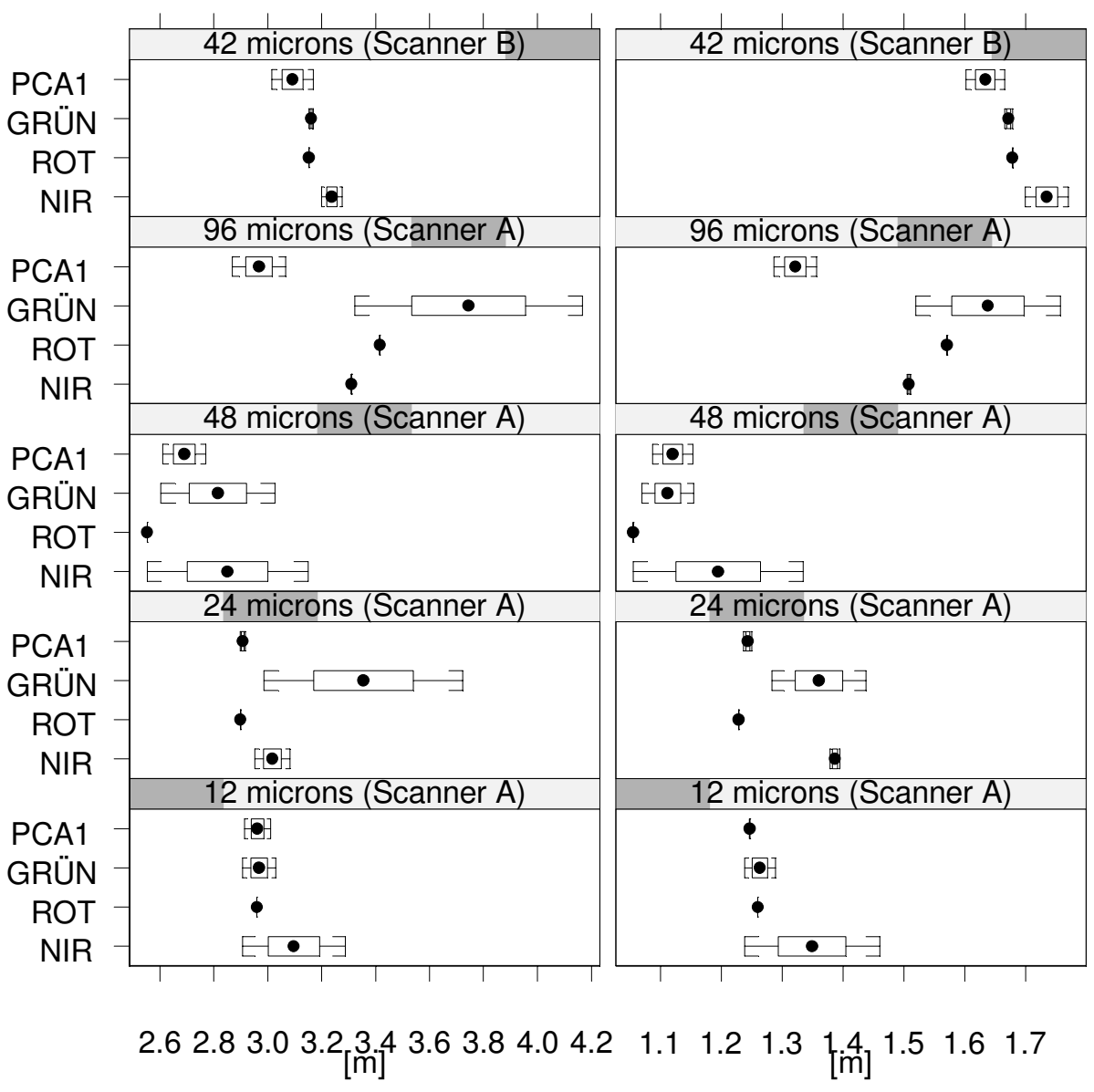

Abb. 4.5: Vergleich der automatisch generierten Höhen des DFS SoftPlotter mit visuell ermittelten Höhen des analytischen Stereoplotters in Abhängigkeit vom Bildkanal und der Scannerauflösung

Die Kistendiagramme in Abb. 4.5 vergleichen RMS-Fehler und mittlere absolute Differenzen der automatisch erstellten Höhenmodelle des DFS SoftPlotter und visuell durchgeführten Messungen am analytischen Stereoplotter. Die Auswahl der ersten Hauptkomponente (PCA1) mit einem Digitalisierungsintervall von 12, 42, und $96 \mu \mathrm{m}$ ist für die automatische Bildzuordnung mit den Bildkanälen NIR, Rot und Grün zu empfehlen. Im Fall der Intervalle 24 und $48 \mu \mathrm{m}$ war die Bildzuordnung von Kanal Rot erfolgreicher. Der Bildkanal NIR weist insgesamt die höchsten Abweichungen auf.

Der Einfluß verschiedener Landbedeckungsklassen auf die Höhengenauigkeit dreier digitaler fotogrammetrischer Systeme geht aus Abb. 4.6 hervor. Die höchsten Genauigkeiten im Vergleich zu visuellen Referenzmessungen des analytischen Stereoplotters weisen die Landbedeckungsklassen 5 (Laubholzkultur), 7 (Acker / Grünland) und 2 (Krone-Licht) auf. Eine Verminderung der Genauigkeit tritt in den Schattenbereichen 
der Baumkronen auf. Die mittleren Fehler im Vergleich zu visuellen Messungen am analytischen Stereoplotter sind in der Klasse 3 (Boden-Lücke) und 4 (Schatten-Lücke) am höchsten (Abb. 4.6). Adler (2001) und Halbritter (1996) berichten ebenfalls über hohe Abweichungen der automatisch generierten Oberflächenmodelle gegenüber visuellen Höhenmessungen in steilen Randbereichen von Waldbeständen.

In Abb. 4.7 sind die RMS-Fehler der Höhenwerte des DFS Softplotter nach Landbedeckung ohne die problematischen Bereiche der Klassen Boden-Lücke und SchattenLücke dargestellt. Geringe Fehler für die Klassen Krone-Licht und Krone-Schatten werden für die Auflösung $48 \mu \mathrm{m}$ des Scanners Wehrli RM2 bestätigt. Zwischen den Medianen der RMS-Fehler der Klasse Krone-Licht sind für die verschiedenen Bildkanäle nur geringe Unterschiede zu erkennen. Die Auswahl des Kanales Rot führt zu geringeren Fehlern in der Klasse Krone-Schatten im Vergleich zu anderen Bildkanälen.

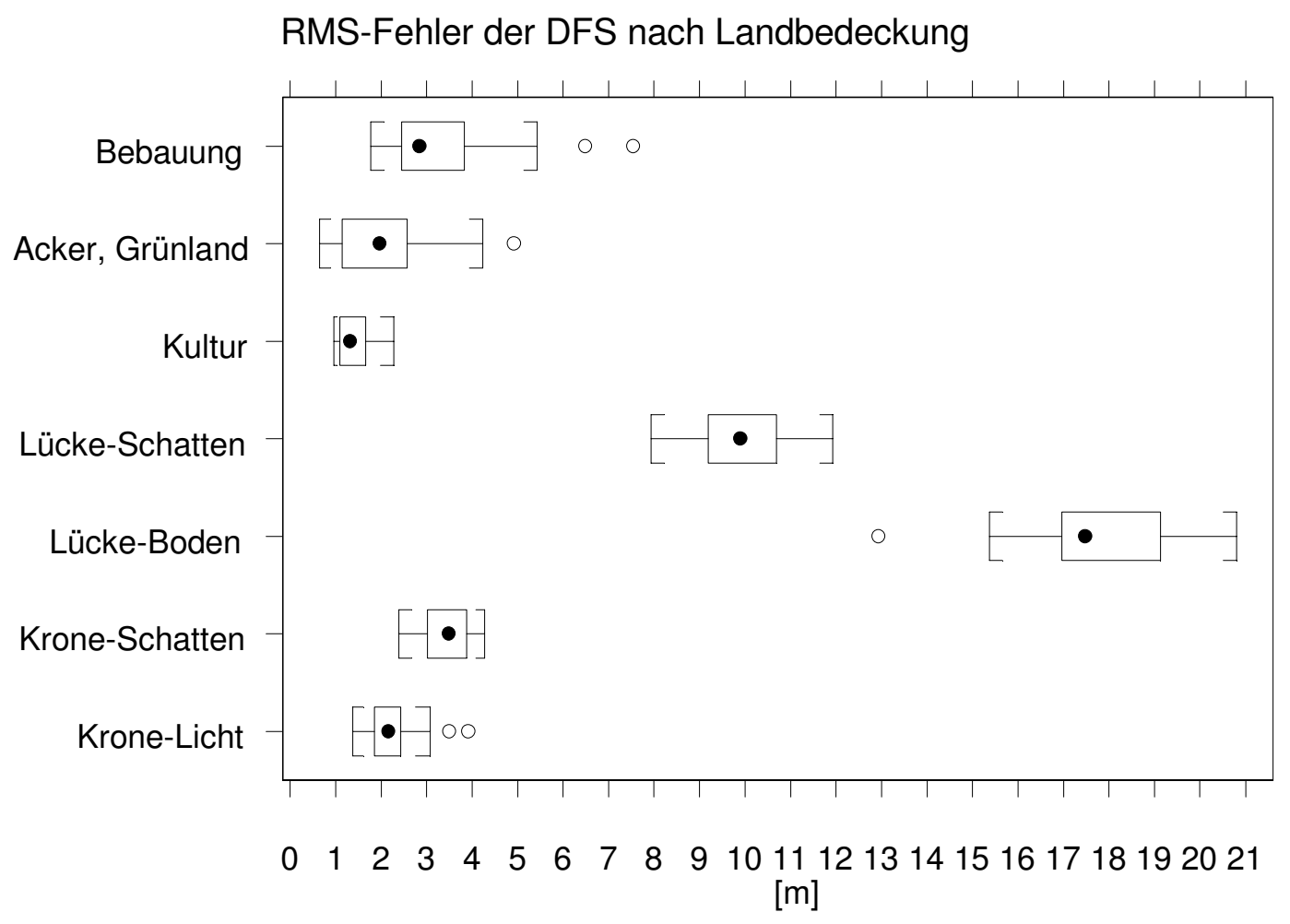

Abb. 4.6: Vergleich der automatisch generierten Höhen von drei DFS mit visuell ermittelten Höhen des analytischen Stereoplotters in Abhängigkeit von der Landbedeckung 
RMS-Fehler des SoftPlotters

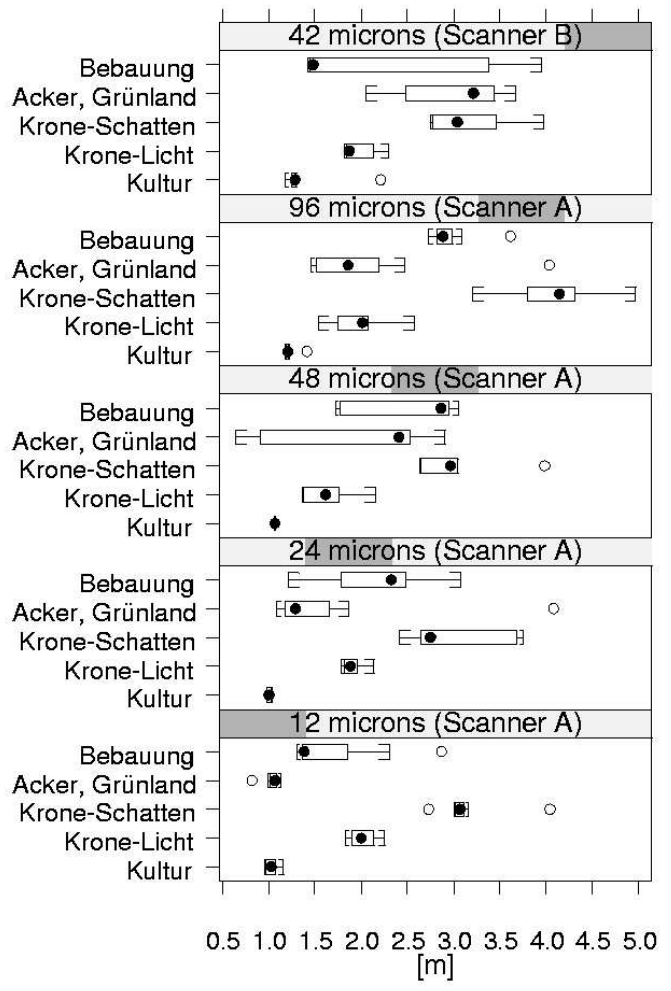

(a)
RMS-Fehler des SoftPlotters

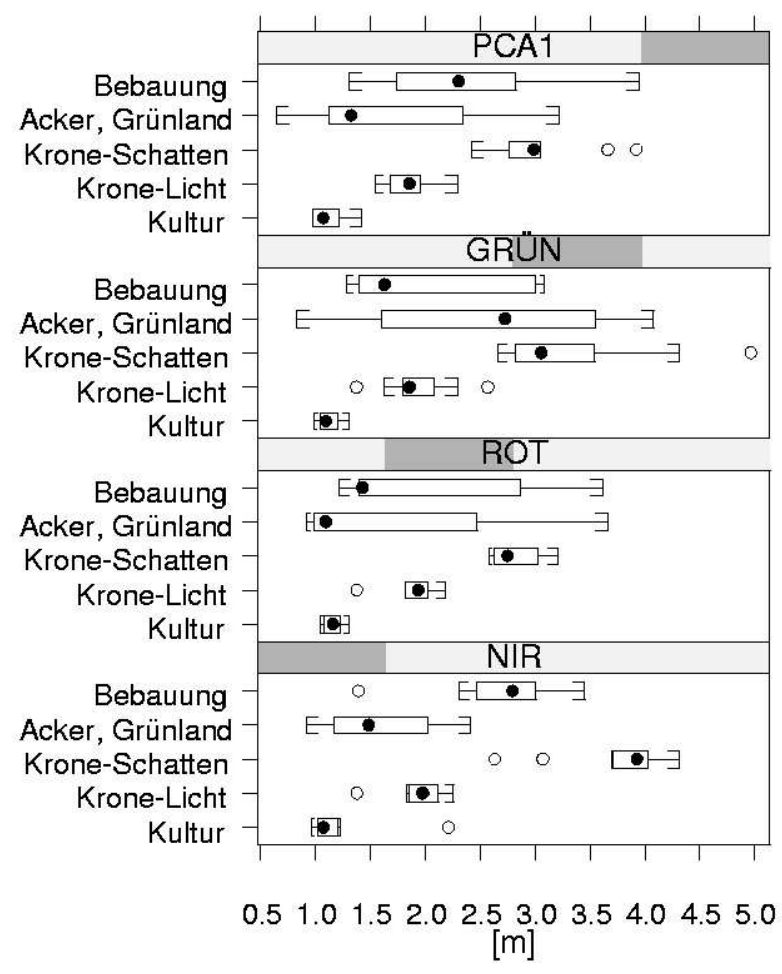

(b)

Abb. 4.7: Vergleich der RMS-Fehler zwischen automatisch erstellten Höhen des DFS SoftPlotter und visuell ermittelten Höhen des analytischen Stereoplotters (a) in Abhängigkeit von Landbedeckung und der Scannerauflösung und (b) in Abhängigkeit der Landbedeckung und des Bildkanals

\subsubsection{Vergleich von Höhendifferenzen im dreifachen Überdeckungsbereich von Stereomodellen}

Die Genauigkeit der automatisch erstellten Höhenmodelle wurde durch den Vergleich von Höhen im mehrfachen Überdeckungsbereich des Bildjahrganges 1988 untersucht. Drei aufeinanderfolgende Bilder werden als L, M, R (Links, Mitte, Rechts) bezeichnet. Es ergeben sich zur Ableitung der Oberflächenmodelle folgende Kombinationsmöglichkeiten der Stereomodelle: LM, MR, LR. Aus den Standardabweichungen der Differenzen LM - LR, MR - LR, LM - MR lassen sich Aussagen zur Höhengenauigkeit ableiten (Sysavath et al. 2001).

Die Standardabweichung der Höhendifferenzen fallen unerwartet hoch aus. In der Gesamtwertung liegen sie bei allen Luftbildmodellen über 1,9 m. Dies ist wahrschein- 
lich auf den geringen Abstand der Projektionszentren der Stereobilder (kleine Bildbasis, Längsüberdeckung ca. 80\%) zurückzuführen.

Die Standardabweichungen der Klasse Krone reichen von 1,5 bis 2,7 m, wobei sich die Klassen Krone-Licht und Krone-Schatten nicht wesentlich unterscheiden (Tab. 4.5). Die geringsten Streuungen der Differenzen treten bei den Klassen Aufforstung, Acker und Grünland und Bebauung auf. Die höchsten Abweichungen sind in der Klasse LückeBoden anzutreffen. In der Klasse Lücke-Schatten gibt es häufig keine Ergebnisse der Bildzuordnung. Die Abweichungen dieser Höhenwerte sind relativ gering, da die Höhen mit einem Interpolationsverfahren aus benachbarten Bildpunkten ermittelt werden, was zu reproduzierbaren Ergebnissen führt.

Im Vergleich sind die Standardabweichungen der automatischen Höhenermittlung der Lichtkronen um den Faktor 2 bis 2,5 größer als die der visuellen Luftbildmessungen am analytischen Stereoplotter (s. Tab. 4.4).

Tab. 4.5: Vergleich der Standardabweichungen (Stdev) bei automatisch erstellten Höhenmodellen (DFS SoftPlotter, Kanal Grün, mit Filterung) zwischen benachbarten Luftbildmodellen (Befliegung 1988, Bildnr. 56, 57, 58)

\begin{tabular}{lc|c|c|c}
\hline & \multicolumn{4}{c}{ Differenzen } \\
\hline & $\mathrm{n}$ & $\begin{array}{c}\text { LM - LR } \\
\text { Stdev [m] }\end{array}$ & $\begin{array}{c}\text { MR - LR } \\
\text { Stdev [m] }\end{array}$ & $\begin{array}{c}\text { LM-MR } \\
\text { Stdev [m] }\end{array}$ \\
\hline Gesamt & 307 & 2,23 & 2,48 & 1,90 \\
1 Krone-Licht & 142 & 2,30 & 2,69 & 1,66 \\
2 Krone-Schatten & 45 & 2,26 & 2,46 & 1,48 \\
3 Boden-Lücke & 3 & 6,79 & 5,96 & 2,10 \\
4 Schatten-Lücke & 8 & 1,82 & 1,51 & 2,98 \\
5 Kultur & 50 & 0,32 & 2,46 & 2,47 \\
6 Acker, Grünland & 42 & 1,01 & 0,73 & 1,49 \\
7 Bebauung & 17 & 2,06 & 1,44 & 2,69 \\
\hline
\end{tabular}

$\mathrm{L}=$ Bild Links, $\mathrm{M}=$ Bild Mitte, $\mathrm{R}=$ Bild Rechts

\section{2 Überschirmungsgrad}

Der Überschirmungsgrad wurde als erster Schritt einer automatischen Segmentierung in den Bildjahrgängen 1988, 1992 und 1999 auf der Fläche 502 bestimmt. Zur Anwendung kamen überwachte Klassifikationsverfahren wie Maximum-Likelihood (ML) und Sequential-Maximum-A-Posteriori (SMAP). Terrestrische Erhebungen des Überschirmungsgrades lagen nicht vor. Es wurden deshalb Trainings- (blau) und Testgebiete 
(grün) einer visuellen Interpretation des digitalen Orthofotos ausgewählt. Sie werden für die Klassifikation 1999 in Abbildung 4.8 (d) dargestellt.

In einer ersten Teilstudie konnten die beiden Klassen Krone und Schatten-Lücke mit einer Gesamtgenauigkeit von 98 - 100\% unterschieden werden. Der SMAP-Klassifkator ergibt höhere oder gleich gute Kappa-Werte wie der ML-Klassifikator. Mit der SMAPKlassifikation entstehen größere zusammenhängende Bildbereiche. Abbildungen 4.8 (a), (b) und (c) stellen einen Ausschnitt der Ergebnisse unter Verwendung der Kanäle Grün, Rot , NIR (nahes Infrarot), NDVI (normalisierter Differenz-Vegetationsindex) und NDOM (normalisiertes Oberflächenmodell) dar. Die Verwendung von fünf Kanälen führt in dieser Fallstudie zu keiner Verbesserung gegenüber drei Kanälen (Grün, Rot, und NIR) des Orthophotos. Der hohe Überschirmungsgrad des Eichen-BuchenBestandes erleichtert die Trennung von Kronendach und Schatten-Lücken aufgrund der spektralen Signaturen. Die Information eines normalisierten Oberflächenmodelles könnte in offenen Waldbeständen mit reflektierender Bodenvegetation von Bedeutung für die Klassifikation des Überschirmungsgrades sein.

Tab. 4.6: Überprüfung der Übereinstimmung der Klassifikation mit der Luftbildinterpretation (2 Klassen: Lücke-Schatten, Krone)

\begin{tabular}{cc|cc|cc|cc|cc}
\hline & & \multicolumn{2}{|c|}{ G-R-NIR } & \multicolumn{2}{c|}{$\begin{array}{c}\text { G-R-NIR } \\
\text {-NDVI }\end{array}$} & \multicolumn{2}{c|}{$\begin{array}{c}\text { G-R-NIR } \\
\text {-NDOM }\end{array}$} & \multicolumn{2}{|c}{$\begin{array}{c}\text { G-R-NIR } \\
\text {-NDVI-NDOM }\end{array}$} \\
\hline Jahr & & OA & Kappa & OA & Kappa & OA & Kappa & OA & Kappa \\
{$[\%]$} & & & & \\
{$[\%]$} & & & \\
\hline 1988 & ML & 99 & 0,96 & 99 & 0,96 & 99 & 0,97 & 99 & 0,97 \\
& SMAP & 100 & 0,98 & 100 & 0,97 & 100 & 0,98 & 100 & 0,97 \\
\hline 1992 & ML & 99 & 0,92 & 98 & 0,85 & 98 & 0,89 & 98 & 0,87 \\
& SMAP & 99 & 0,93 & 98 & 0,90 & 99 & 0,95 & 99 & 0,93 \\
\hline 1999 & ML & 98 & 0,87 & 99 & 0,90 & 98 & 0,89 & 99 & 0,90 \\
& SMAP & 99 & 0,91 & 99 & 0,91 & 99 & 0,92 & 99 & 0,92 \\
\hline
\end{tabular}

Bildkanal: $\mathrm{G}=$ Grün, $\mathrm{R}=$ Rot, NIR = nahes Infrarot, NDVI = normalisierter DifferenzVegetationsindex, NDOM = normalisiertes digitales Oberflächenmodell, OA = Gesamtgenauigkeit, Kappa = Kappa-Maß

Die Ergebnisse der Klassifikation der zweiten Teilstudie kennzeichnen Gesamtgenauigkeiten von 57 - 74\% (s. Tab. 4.7). Die Definition der Klasse Krone-Schatten führt zu teilweise unbefriedigenden Ergebnissen, die zum Rand des Bildausschnittes hin zunehmen. Die Klassifikation mit SMAP führt auch hier zu einer besseren oder gleich guten Übereinstimmung mit der visuellen Interpretation als die ML-Klassifikation.

Die Veränderungen des Überschirmungsgrades in den Jahren 1988, 1992 und 1999 von zwei Teilflächen (BDF und Level 2, Fläche 502) werden als Ergebnis von drei 


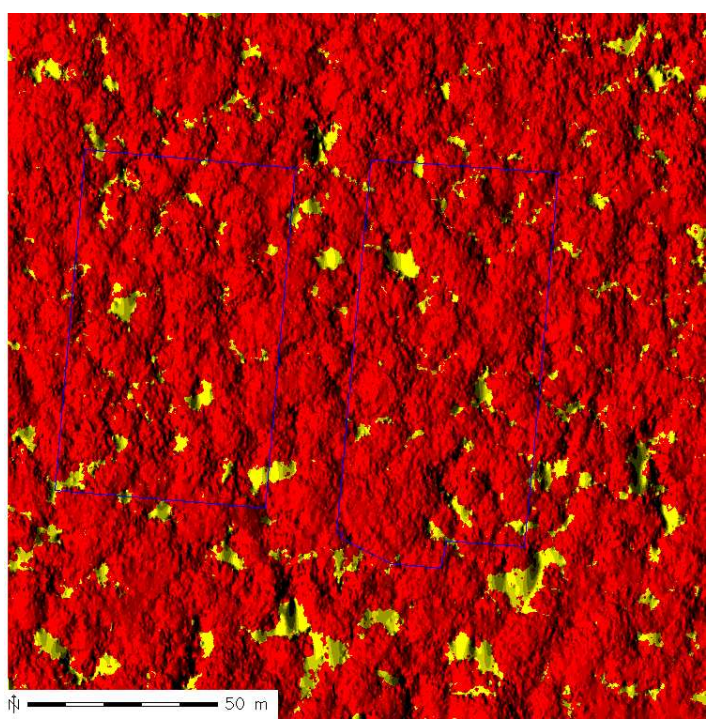

(a) Klassifikation SMAP 1988

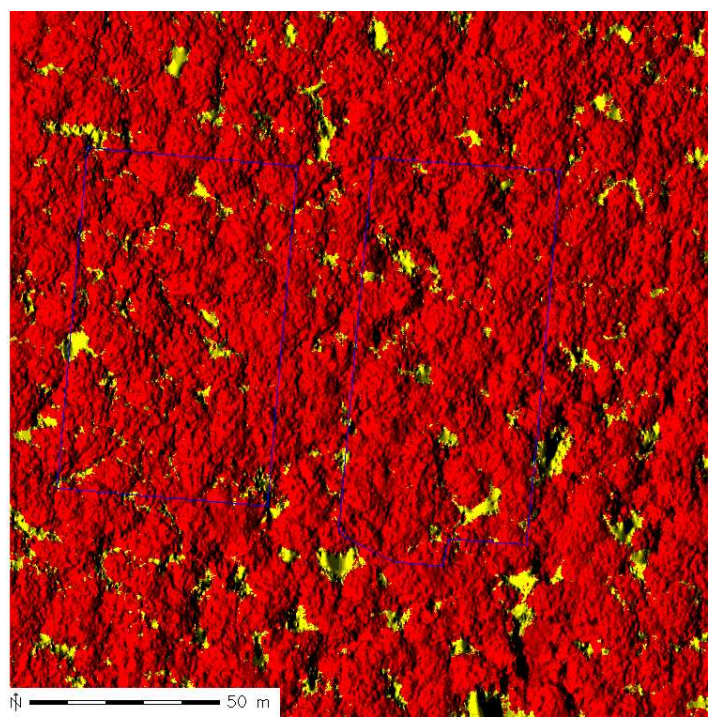

(c) Klassifikation SMAP 1999

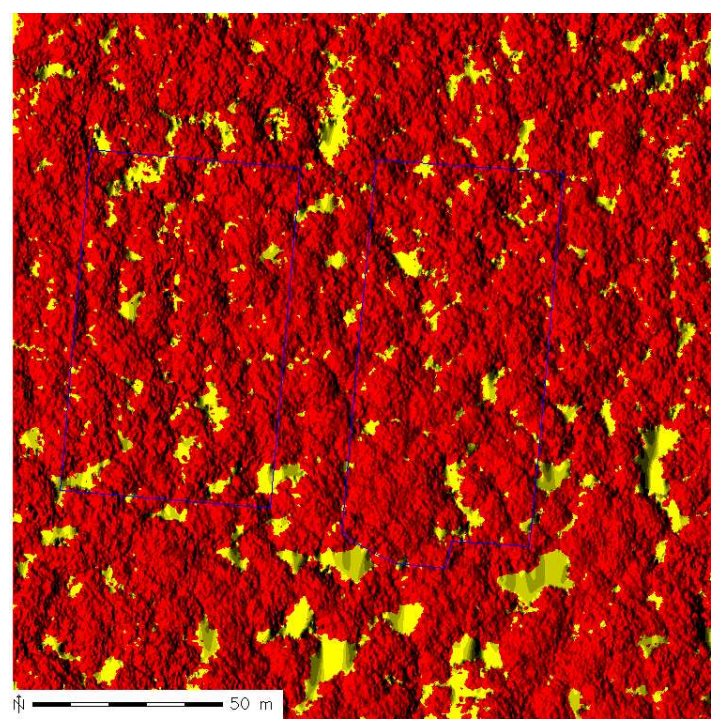

(b) Klassifikation SMAP 1992

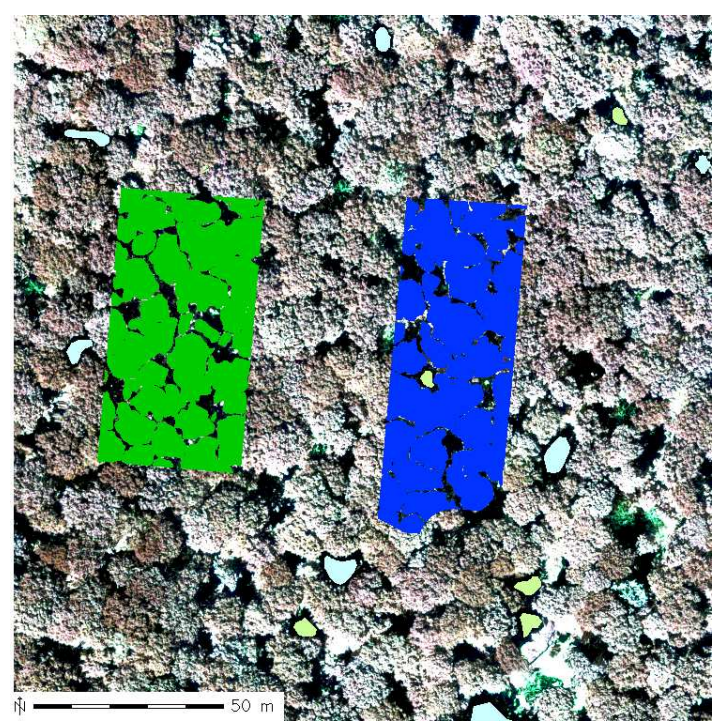

(d) Trainings- (blau) und Testgebiete (grün) im Orthophoto 1999

Abb. 4.8: Ergebnisse der SMAP-Klassifikation von Krone (rot) und Schatten-Lücke (gelb) mit 4 Kanälen (Grün, Rot, NIR und NDOM) (a) 1988, (b) 1992 und (c) 1999, (d) Trainings- (blau) und Testgebiete (grün) überlagert über digitalem Orthofoto 1999. 
Tab. 4.7: Überprüfung der Übereinstimmung der Klassifikation mit der Luftbildinterpretation (3 Klassen: Lücke-Schatten, Krone-Licht, Krone-Schatten)

\begin{tabular}{cc|cc|cc|cc|cc}
\hline & \multicolumn{2}{|c|}{ G-R-NIR } & \multicolumn{2}{c|}{$\begin{array}{c}\text { G-R-NIR } \\
\text {-NDVI }\end{array}$} & \multicolumn{2}{c|}{$\begin{array}{c}\text { G-R-NIR } \\
\text {-NDOM }\end{array}$} & \multicolumn{2}{c}{$\begin{array}{c}\text { G-R-NIR } \\
\text { NDVI-NDOM }\end{array}$} \\
\hline & & $\begin{array}{c}\text { OA } \\
{[\%]}\end{array}$ & Kappa & OA & Kappa \\
{$[\%]$} & & OA & Kappa \\
{$[\%]$} & & $\begin{array}{c}\text { OA } \\
{[\%]}\end{array}$ & Kappa \\
\hline 1988 & ML & 70 & 0,43 & 66 & 0,37 & 67 & 0,40 & 67 & 0,38 \\
& SMAP & 74 & 0,47 & 72 & 0,45 & 69 & 0,42 & 70 & 0,43 \\
\hline 1992 & ML & 69 & 0,43 & 69 & 0,38 & 57 & 0,33 & 63 & 0,35 \\
& SMAP & 69 & 0,43 & 73 & 0,45 & 60 & 0,36 & 64 & 0,37 \\
\hline 1999 & ML & 65 & 0,36 & 66 & 0,36 & 67 & 0,40 & 60 & 0,34 \\
& SMAP & 66 & 0,39 & 71 & 0,44 & 67 & 0,41 & 63 & 0,38 \\
\hline
\end{tabular}

Bildkanal: $\mathrm{G}=$ Grün, $\mathrm{R}=$ Rot, NIR = nahes Infrarot, NDVI = normalisierter DifferenzVegetationsindex, NDOM = normalisiertes digitales Oberflächenmodell, OA = Gesamtgenauigkeit, Kappa = Kappa-Maß

methodischen Varianten vorgestellt (Tab. 4.8). Die erste Variante als Ergebnis der digitalen Klassifikation (SMAP) erzielt die höchsten Überschirmungsgrade. Die visuelle Kronenkartierung mit vollständiger Berücksichtigung der Kronenschirmflächen von Bäumen innerhalb der Untersuchungsfläche (Vis. 1) und die visuelle Kronenkartierung einer kleineren Teilfläche (Vis. 2) sind erheblich geringer im Vergleich zur digitalen Klassifikation. Die visuelle Kartierung von Einzelkronen unterschätzt den Überschirmungsgrad um ca. 10 - $20 \%$. Die Abweichungen entstehen durch Kronenteile im Schatten oder in der Unterschicht, die während der visuellen Abgrenzung von Einzelkronen in der Bestandesoberschicht nicht eindeutig zugeordnet werden können. Münch (1995) stellt eine visuelle Luftbildkartierung der Standflächen von Baumkronen vor, die Schatten zwischen den Kronen einbezieht. Diese Auswertung wird mit einer Kartierung der Bestandeslücken verglichen, die als Öffnungen im Kronendach mit einer Mindestfläche und relativen Höhe der umstehenden Bäume definert werden. Die visuelle Standflächenkartierung der Baumkronen ergab ebenfalls eine höhere Lückenfläche bzw. einen geringeren Überschirmungsgrad als die Kartierung der Bestandeslücken Münch (1995).

Der Fehler durch Ausschluß der Kronenschirmflächen von Randbäumen beim visuellen Verfahren Vis. 1 wird demgegenüber als gering eingeschätzt wie ein Vergleich der Überschirmungsgrade zwischen den visuellen Kronenkartierungen Vis. 1 und Vis. 2 zeigt (Tab. 4.8). 
Tab. 4.8: Änderungen des Überschirmungsgrades am Beispiel von zwei Untersuchungsflächen BDF und Level 2

\begin{tabular}{c|cc|cc|cc|cc|cc|cc}
\hline Jahr & \multicolumn{5}{|c|}{ BDF } & \multicolumn{5}{c}{ Level2 } \\
\hline Jahr & \multicolumn{2}{|c|}{ Vis.1 } & \multicolumn{2}{|c|}{ Vis.2 } & \multicolumn{2}{c|}{ SMAP } & \multicolumn{2}{c|}{ Vis.1 } & \multicolumn{2}{c}{ Vis.2 } & \multicolumn{2}{c}{ SMAP } \\
& $m^{2}$ & $\%$ & $m^{2}$ & $\%$ & $m^{2}$ & $\%$ & $m^{2}$ & $\%$ & $m^{2}$ & $\%$ & $m^{2}$ & $\%$ \\
\hline 1988 & 3738 & 73,1 & 2004 & 72,4 & 4807 & 94,0 & 3849 & 74,4 & 2035 & 75,3 & 4896 & 94,7 \\
1992 & 4032 & 78,8 & 2169 & 78,3 & 4645 & 91,8 & 4166 & 80,6 & 2174 & 80,4 & 4806 & 93,0 \\
1999 & 4098 & 80,1 & 2200 & 79,5 & 4802 & 93,9 & 4059 & 78,5 & 2154 & 79,7 & 4518 & 87,4 \\
Ges. & 5115 & 100 & 2769 & 100 & 5115 & 100 & 5170 & 100 & 2703 & 100 & 5170 & 100 \\
\hline
\end{tabular}

Vis. 1: Visuelle Auswertung, Summe der Kronenschirmflächen aller Bäume mit Baumfüßen innerhalb der Fläche

Vis. 2: Visuelle Auswertung, Summe der Kronenschirmflächen mit Baumfüßen innerhalb u. außerhalb einer kleineren Teilfläche

SMAP: digitale Klassifikation mit SMAP, Fläche der Klasse Krone

\subsection{Segmentierung}

Der Eichen-Buchen-Bestand 502 wurde als Testfläche für eine automatische Segmentierung ausgewählt. Regionen der Klasse Lücke-Schatten als Ergebnis der überwachten Klassifikation des Überschirmungsgrades wurden im normalisierten digitalen Oberflächenmodell der Befliegung 1999 als Bildhintergrund kodiert. Nach Invertierung der Höhenwerte wurde das normalisierte Oberflächenmodell mit Gaußschen Filterkernen verschiedener Fenstergrößen und Standardabweichungen geglättet. Abb. 4.9 stellt das Ergebnis des Wasserscheiden-Algorithmus (GRASS-Programm r.terraflow) mit der Option der Mehrwege-Fließrichtung als Segmentierung der Baumkronen vor. Nach mehreren Iterationen wurde eine Filtergröße von $15 \times 15$ Pixeln und eine Standardabweichung von $\sigma=7$ gewählt. Zur visuellen Überprüfung wurde das Orthofoto mit den Wasserscheiden überlagert (Abb. 4.9 (a)).

Abb. 4.9 (b) zeigt einen vergrößerten Bildausschnitt der Level 2-Fläche 502. Die grünen Vektoren der Kronenränder werden als Ergebnis der visuellen Stereokartierung am SoftPlotter dargestellt. Die Baumkronen wurden während der visuellen Kartierung mit einem terrestrisch Stammverteilungsplan verglichen. Die roten Vektoren sind das Ergebnis der automatischen Segmentierung nach dem Wasserscheidenverfahren. Bei der Segmentierung entstehen zum Teil sehr kleine Polygone. Polygone, die eine Mindestfläche von $2 \mathrm{~m}^{2}$ unterschreiten, wurden aufgelöst und angrenzenden Polygonflächen zugeteilt.

Tab. 4.9 vergleicht das Ergebnis der visuellen mit der digitalen Segmentierung auf der Testfläche Level 2. Das Ergebnis der automatischen Segmentierung unterschätzt die Kronenzahl um $20 \%$ und überschätzt die mittlere Kronenschirmfläche um $47 \%$. 


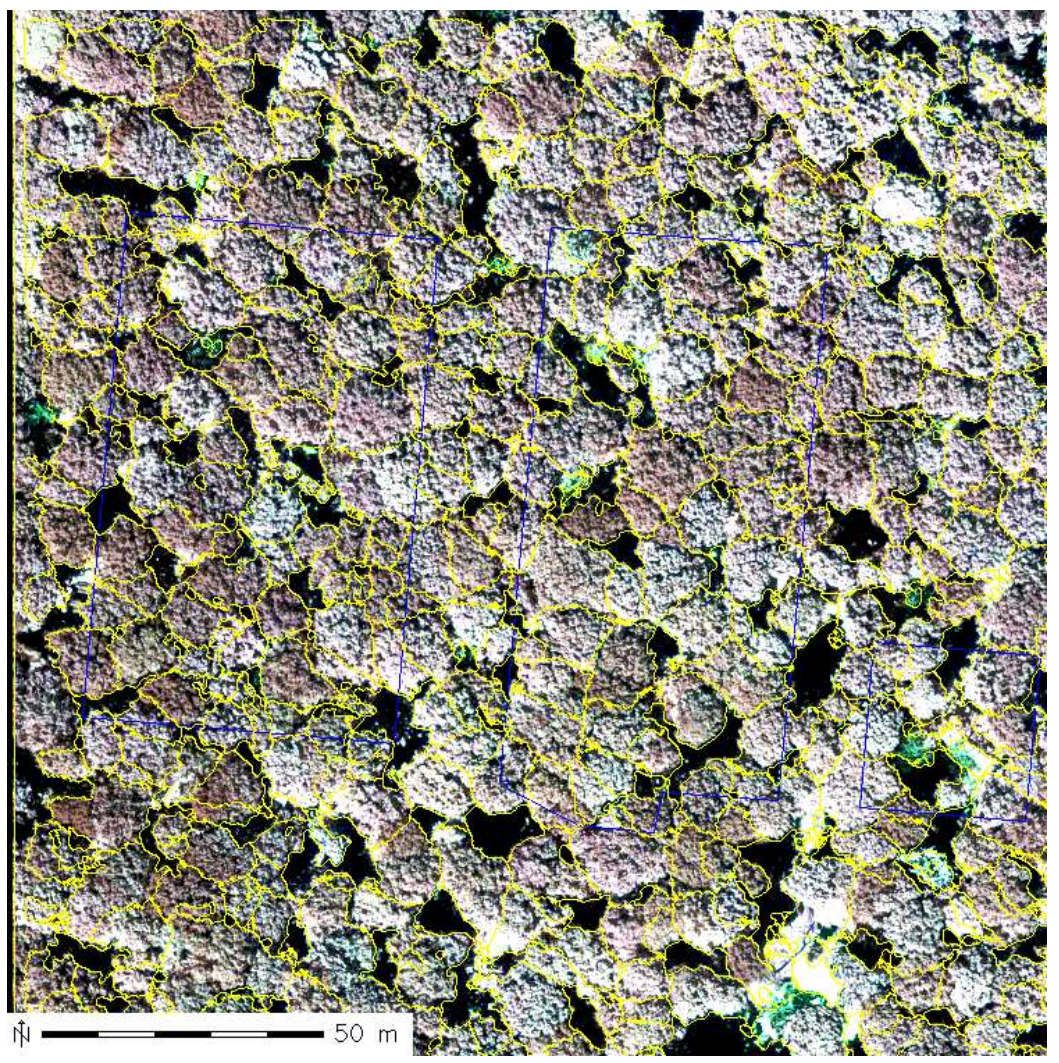

(a) Digitales Orthophoto 1999 überlagert mit Segmentgrenzen des Wasserscheiden-Algorithmus (gelb)

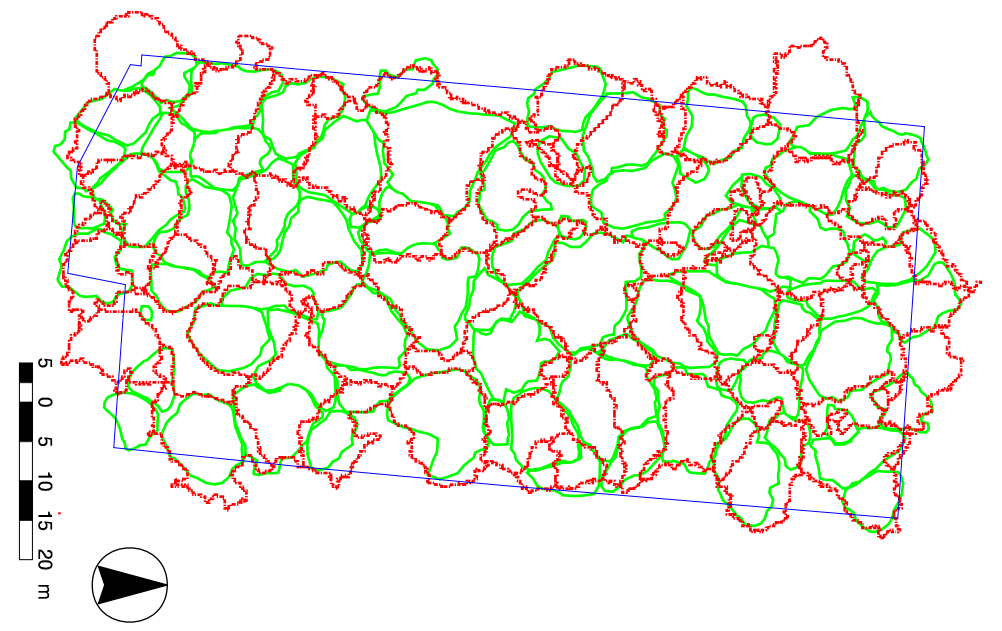

(b) Vergleich der visuellen (grün) mit der automatischen Segmentierung (rot) der Baumkronen auf der Fläche 502, Level 2, 1999

Abb. 4.9: Ergebnis der Segmentierung mit dem Wasserscheidenverfahren, Fläche 502, Bildflug 1999 
Die Abweichungen des automatischen Verfahrens entstehen überwiegend durch das Nichterkennen von Kronenrändern zwischen Baumkronen, die sich berühren und zusammenballen. In diesen Fällen sollte eine Segmentierung durch Berücksichtigung zusätzlicher Baummerkmale durchgeführt werden. Das Ergebnis der automatischen Segmentierung ist stark abhängig von der Größe und Standardabweichung des Gaußschen Filterkerns bei der Glättung des Oberflächenmodelles.

Tab. 4.9: Genauigkeit der automatischen Segmentierung im Vergleich zur visuellen fotogrammetrischen Auswertung

\begin{tabular}{cccccc}
\hline \multicolumn{5}{c}{ Kronenschirmfläche KSF } \\
\hline & $\mathrm{N}$ & Mean $\left[\mathrm{m}^{2}\right]$ & $\mathrm{Stdev}\left[\mathrm{m}^{2}\right]$ & $\operatorname{Min}\left[\mathrm{m}^{2}\right]$ & $\operatorname{Max}\left[\mathrm{m}^{2}\right]$ \\
\hline Visuelle Auswertung & 80 & 51,5 & 27,6 & 2,1 & 163,2 \\
Automatische Segmentierung & 64 & 75,6 & 52,6 & 2,1 & 216,8 \\
\hline
\end{tabular}

\subsection{Farb- und Formmerkmale von Baumkronen}

\subsubsection{Farbmerkmale im digitalen Orthofoto}

Die Baumkronen auf der Fläche 502 werden mit einer visuellen Stereoauswertung des digitalen fotogrammetrischen Systems SoftPlotter kartiert. Die Bildpunkte innerhalb der visuell abgegrenzten Baumkronen werden zu Segmenten zusammengefasst, die mit verschiedenen Merkmalen näher beschrieben werden können. Damit findet ein Übergang von einer pixelbasierten zu einer listenorientierten Bildverarbeitung statt. Zur Klassifikation der Segmente sind in einer Voruntersuchung Merkmale zu bestimmen, die eine möglichst gute Unterscheidung der Segmente erlauben.

Die spektralen Reflexionseigenschaften der Kronensegmente werden in einer Signaturanalyse am Beispiel des digitalen Orthofotos 1988 untersucht. Grundlage sind 3 RGB-Kanäle (Grün, Rot, NIR), der Normalisierte Differenz-Vegetationsindex (NDVI), die erste und zweite Hauptkomponente (PC1 und PC2). Die Kanäle NDVI, PC1, PC2 werden linear auf eine 8-Bit-Auflösung transformiert. Zusätzlich werden die Texturmerkmale contrast und variance einer Grauwertübergangsmatrix des Spektralbereiches des nahen Infrarot berücksichtigt.

Zunächst wird die Möglichkeit der Baumartentrennung anhand der Mittelwerte der segmentbasierten Mittelwerte und Standardabweichungen von Farb- und Formmerkmalen am Beispiel von 231 Eichen und 44 Buchen auf der Fläche 502 untersucht. In einem zweiten Schritt werden die spektralen und geometrischen Signaturen der Kronenverlichtungsstufen der Eichen charakterisiert. Als Referenz stehen terrestrische Einschätzungen des Blattverlustes von 81 Eichen zur Verfügung. 

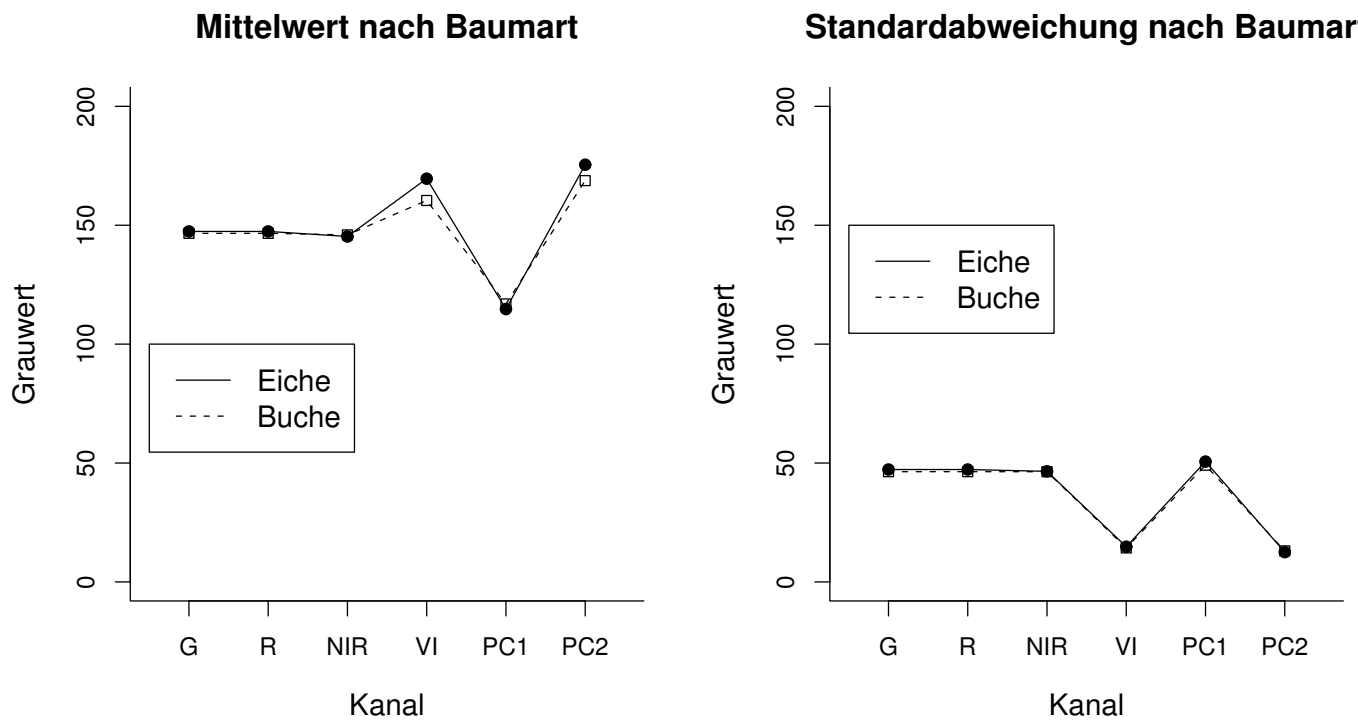

(a) Spektrale Signaturen von Eichen und Buchen, Fläche 502, 1988

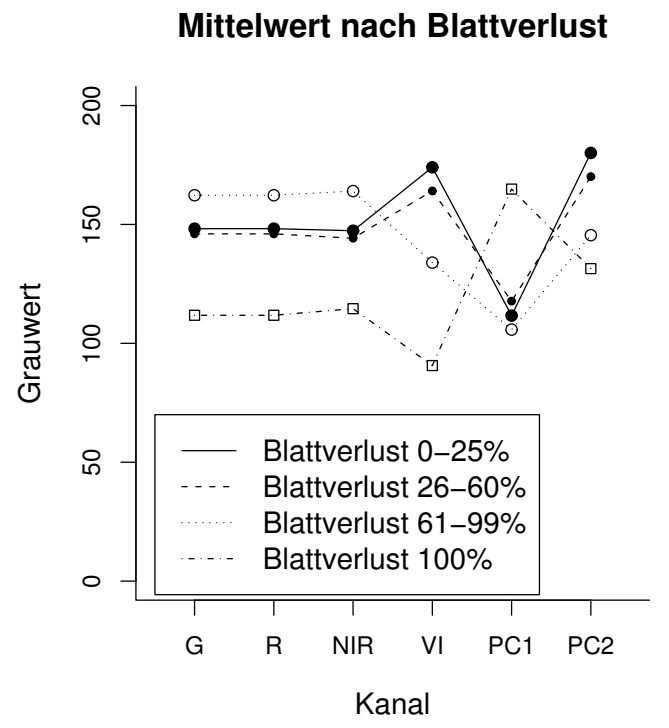

\section{Standardabweichung nach Blattverlust}

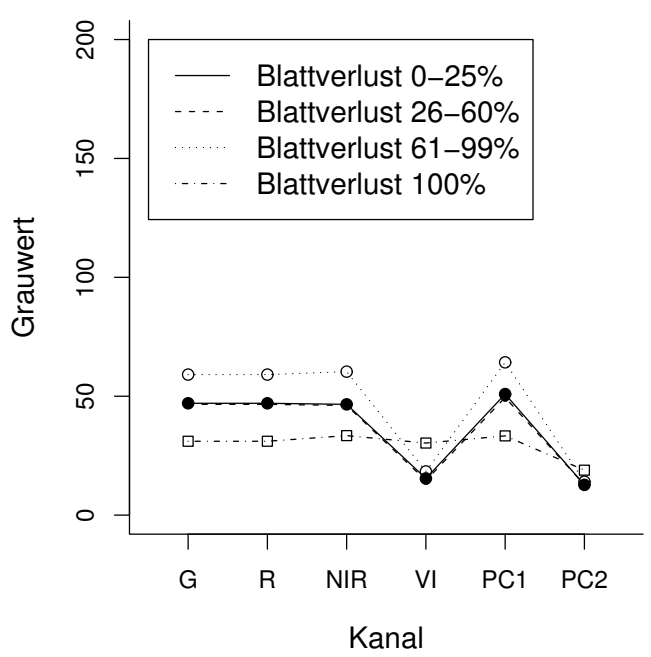

(b) Spektrale Signaturen der Blattverluststufen von Eichen, Fläche 502, 1988

Abb. 4.10: Spektrale Signaturen im Orthofoto 1988 (Mittelwerte der baumkronenspezifischen Mittelwerte und Standardabweichungen) ( $\mathrm{G}=$ Grün, $\mathrm{R}=\mathrm{Rot}, \mathrm{NIR}$ = nahes Infrarot, VI = NDVI-Vegetationsindex, PC1 und PC2 = Hauptkomponenten) 
Die Verbindungslinien in den Diagrammen stellen keinen funktionellen Zusammenhang dar, sondern dienen der anschaulichen Darstellung der Unterschiede zwischen Baumarten und Blattverluststufen.

Eine Trennung der Baumarten Eiche und Buche ist nach Abb. 4.10 mit Hilfe der ursprünglichen Kanäle Grün, Rot, nahes Infrarot und der ersten Hauptkomponente des Orthofotos nicht möglich. Die Kanäle NDVI und PC2 zeigen sehr geringe Unterschiede zwischen den Mittelwerten von Eichen und Buchen. Die mittlere Standardabweichung bietet keinen zusätzlichen Informationsgehalt zur Baumartentrennung (Abb. 4.10 (a)).

Die Mittelwerte der Blattverluststufen lassen eine Differenzierung der Stufen B3 (61 - $99 \%)$ und B4 (100 \%) in allen spektralen Kanälen zu. Die Trennung der Blattverluststufen B1 (0 - 25 \%) und B2 (25-60\%) ist dagegen unter ausschließlicher Verwendung der Kanäle Grün, Rot und NIR nicht möglich. Die Unterschiede der Mittelwerte nach Blattverluststufen sind in den Kanälen NDVI und PC2 am größten. Sie sind außerdem durch relativ geringe mittlere Standardabweichungen innerhalb der Kronensegmente gekennzeichnet (Abb. 4.10 (b)). Mit zunehmendem Blattverlust sinken die Werte des NDVI und der zweiten Hauptkomponente PC2.

Die Unterschiede der Mittelwerte der Texturmerkmale contrast und variance sind am höchsten im Bereich des nahen Infrarot im Vergleich zum grünen und roten Spektralbereich. Buchen zeigen etwas geringere Mittelwerte der Texturen contrast und variance im Vergleich zu den Eichen. Beide Texturmerkmale sinken bei größeren Blattverlusten der Eichen. Dies kann als eine zunehmend diffuse und unscharfe Darstellung der Baumkronen bei fortschreitender Entlaubung interpretiert werden. Contrast und variance sind straff miteinander korreliert (Abb. 4.11).

Mittelwert nach Baumart

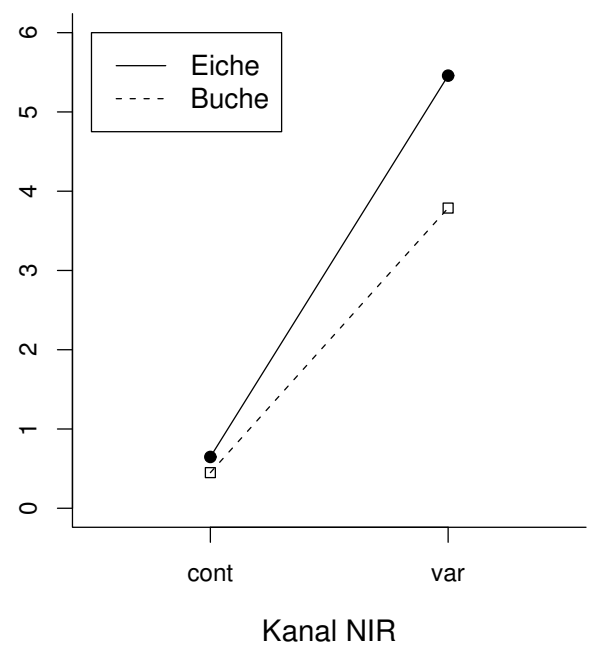

Mittelwert nach Blattverlust

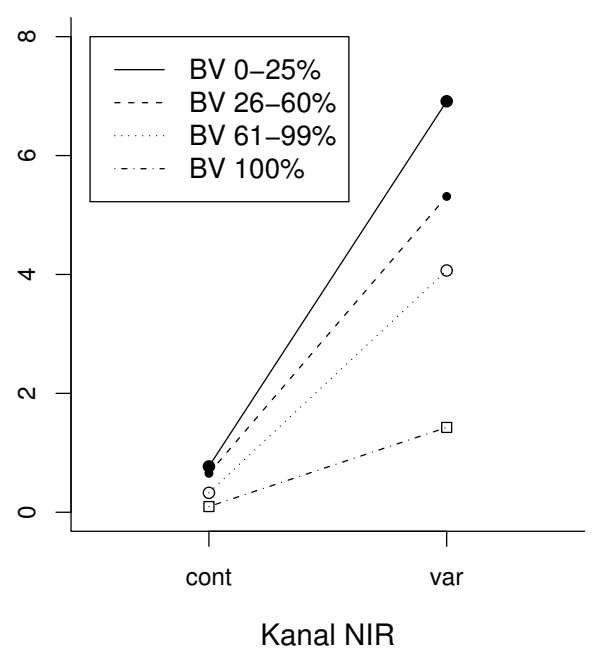

Abb. 4.11: Texturmerkmale contrast (cont) und variance (var) im spektralen Bereich des nahen Infrarot im Orthofoto 1988 


\subsubsection{Kronenbreite, -länge, -schirmfläche, -mantelfläche und -volumen}

Die Erfassung der Kronendimensionen werden im Folgenden als Ergebnisse von Zeitreihenauswertungen dargestellt. Die Veränderung der Mittelwerte und Standardabweichungen in Untersuchungszeiträumen von 3 bis 12 Jahren können aus tabellarischen Übersichten für Eichen, Buchen und Fichten entnommen werden.

Tab. 4.10: Ergebnisse der visuellen Bildauswertung als Mittelwert (Mean) und Standardabweichung (Stdev) der Kronenbreite $(K B)$, Kronenschirmfläche $(K S F)$, Kronenmantelfläche $(K M F)$ und des Kronenvolumens $(K V O L)$ auf den Eichenflächen

\begin{tabular}{|c|c|r|r|r|r|r|r|rr|r|r|}
\hline & & \multicolumn{10}{|c|}{ Eiche } \\
\hline & Fl.Nr. & \multicolumn{2}{|c|}{405} & \multicolumn{2}{|c|}{ WB0 } & \multicolumn{2}{|c|}{ WBK } & \multicolumn{3}{c|}{502} \\
\hline & Jahr & 1992 & 1999 & 1992 & 1999 & 1992 & 1999 & 1988 & 1992 & 1999 \\
\hline & $\mathrm{N}$ & 51 & 46 & 39 & 34 & 37 & 37 & 192 & 192 & 192 \\
\hline$K B$ & Mean & 7,0 & 6,9 & 7,0 & 7,2 & 7,5 & 7,5 & 8,1 & 8,5 & 9,0 \\
{$[m]$} & Stdev & 1,6 & 1,2 & 1,3 & 1,4 & 1,7 & 1,7 & 2,1 & 2,2 & 2,3 \\
\hline$K L$ & Mean & 4,2 & 5,1 & 4,1 & 4,5 & 4,8 & 4,2 & 3,6 & 4,1 & 5,0 \\
{$[m]$} & Stdev & 1,2 & 1,7 & 1,0 & 1,1 & 1,3 & 1,0 & 1,0 & 1,1 & 1,5 \\
\hline$K S F$ & Mean & 41,0 & 39,5 & 40,7 & 42,4 & 45,4 & 45,8 & 55,3 & 60,8 & 67,3 \\
{$\left[m^{2}\right]$} & Stdev & 19,1 & 19,6 & 15,2 & 15,7 & 18,9 & 19,5 & 27,3 & 29,7 & 32,3 \\
\hline$K M F$ & Mean & 101,6 & 129,9 & 97,5 & 109,2 & 121,3 & 104,3 & 98,5 & 117,5 & 153,0 \\
{$\left[m^{2}\right]$} & Stdev & 44,2 & 69,1 & 32,9 & 42,2 & 48,5 & 44,3 & 45,2 & 50,6 & 73,4 \\
\hline$K V O L$ & Mean & 141,4 & 196,9 & 128,6 & 154,6 & 180,3 & 147,4 & 136,5 & 177,9 & 264,4 \\
{$\left[m^{3}\right]$} & Stdev & 90,0 & 148,2 & 62,2 & 87,0 & 105,6 & 96,2 & 94,9 & 113,2 & 186,3 \\
\hline$C R$ & Mean & 2,5 & 3,3 & 2,4 & 2,6 & 2,7 & 2,3 & 1,8 & 1,9 & 2,3 \\
\hline
\end{tabular}

Kronenrauhigkeit $C R=K M F / K S F$

In Tab. 4.10 werden die Ergebnisse für die untersuchten Eichenflächen zusammengefasst. Die mittleren Kronenschirmflächen der Eichen auf den Teilflächen 405, WBK und WBO sind innerhalb der siebenjährigen Untersuchungsperiode gleich geblieben oder nur geringfügig gestiegen. Dagegen steigt die mittlere Kronenschirmfläche auf der Fläche 502 kontinuierlich an. Die mittlere Kronenlänge wird durch die Bestandesdichte beeinflusst. Das Beispiel der Flächen WBO und 405 zeigt, dass die Entnahme von fünf bzw. sechs Bäumen im Jahr 1999 zu einer erheblich größeren Kronenlänge führt als 1992. Mantelfläche und Volumen der Kugelschnitte vergrößern sich ebenfalls. Auf der WBK-Fläche wurden dagegen keine Bäume entnommen. Die mittlere Kronen- 
länge ist am Ende der Untersuchungsperiode kleiner als am Anfang. Hieraus ergeben sich ebenfalls geringere Kronenmantelflächen und -volumina.

Die mittlere Kronenrauhigkeit als Verhältnis zwischen Kronenmantelfläche und Kronenschirmfläche ist mit Ausnahme der Fläche WBK am Ende des Untersuchungszeitraumes größer als am Anfang und liegt bei den untersuchten Altbeständen zwischen 1,8 und 3,3.

Die Mittelwerte der Kronengrößen der Buchen beschreiben einen kontinuierlichen Anstieg in den zwölfjährigen Untersuchungszeiträumen (Tab. 4.11). Eine Ausnahme bildet auch hier die mittlere Kronenlänge, die größeren Schwankungen in der Zeitreihenentwicklung unterliegt, was nicht durch Veränderungen der Bestandesdichte erklärt werden kann. Die mittlere Kronenrauhigkeit ist am geringsten in dem ca. 150-jährigen Buchenaltbestand und liegt zwischen 1,6 und 2,6.

Tab. 4.11: Ergebnisse der visuellen Bildauswertung als Mittelwert (Mean) und Standardabweichung (Stdev) der Kronenbreite $(K B)$, Kronenschirmfläche $(K S F)$, Kronenmantelfläche $(K M F)$, Kronenvolumen $(K V O L)$ der Buchenflächen

\begin{tabular}{|c|c|r|r|r|r|r|r|r|}
\hline & & \multicolumn{6}{|c|}{ Buche } \\
\hline & Fl.Nr. & \multicolumn{6}{|c|}{203} & \multicolumn{3}{|c|}{502} \\
\hline & Jahr & 1984 & 1987 & 1992 & 1995 & 1988 & 1992 & 1999 \\
\hline & $\mathrm{N}$ & 31 & 31 & 31 & 31 & 20 & 20 & 20 \\
\hline$K B$ & Mean & 9,2 & 9,5 & 10,0 & 10,8 & 6,1 & 6,2 & 7,2 \\
{$[m]$} & Stdev & 1,6 & 1,6 & 1,8 & 1,8 & 2,5 & 2,9 & 3,2 \\
\hline$K L$ & Mean & 2,6 & 2,9 & 2,7 & 4,4 & 3,4 & 3,6 & 4,5 \\
{$[m]$} & Stdev & 0,7 & 0,8 & 1,0 & 1,5 & 1,5 & 1,2 & 1,9 \\
\hline$K S F$ & Mean & 57,9 & 61,2 & 66,3 & 77,1 & 34,6 & 36,9 & 48,9 \\
{$\left[m^{2}\right]$} & Stdev & 22,8 & 21,8 & 22,0 & 25,5 & 26,0 & 30,4 & 37,4 \\
\hline$K M F$ & Mean & 90,4 & 101,1 & 105,8 & 161,5 & 77,9 & 82,8 & 124,9 \\
{$\left[m^{2}\right]$} & Stdev & 28,0 & 31,7 & 33,8 & 63,0 & 53,3 & 49,9 & 84,0 \\
\hline$K V O L$ & Mean & 99,9 & 123,1 & 125,2 & 271,7 & 102,2 & 110,0 & 209,2 \\
{$\left[m^{3}\right]$} & Stdev & 47,7 & 61,6 & 66,1 & 164,7 & 93,3 & 89,2 & 184,4 \\
\hline$C R$ & Mean & 1,6 & 1,7 & 1,6 & 2,1 & 2,3 & 2,2 & 2,6 \\
\hline
\end{tabular}

Kronenrauhigkeit $C R=K M F / K S F$

Im Fall der Fichtenflächen 130 - 133 wurden nach fünf Jahren kleinere Kronendimensionen ermittelt als im Jahr 1992. Auch auf der Fichtenfläche 108 wurden im Jahr 1986 auffallend geringe Kronenlängen gemessen (Tab. 4.12). Diese Ergebnisse sind auf den ungünstigen Einfluss eines niedrigen Sonnenzenitwinkels (ca. $40^{\circ}$ ) zum Zeitpunkt der Bildaufnahme am 22.9.1997 und 25.9.1986 zurückzuführen. 
Tab. 4.12: Ergebnisse der visuellen Bildauswertung als Mittelwert (Mean) und Standardabweichung (Stdev) der Kronenbreite $(K B)$, Kronenschirmfläche $(K S F)$, Kronenmantelfläche $(K M F)$ und des Kronenvolumens $(K V O L)$ auf den Fichtenflächen. Kronenrauhigkeit $C R=K M F / K S F$.

\begin{tabular}{|c|c|rrrr|}
\hline & & \multicolumn{4}{|c|}{ Fichte } \\
\hline & Fl.Nr. & \multicolumn{4}{|c|}{108} \\
\hline & Jahr & 1984 & 1986 & 1992 & 1995 \\
\hline & $\mathrm{N}$ & 165 & 165 & 112 & 111 \\
\hline$K B$ & Mean & 3,1 & 3,0 & 3,8 & 4,1 \\
{$[m]$} & Stdev & 0,7 & 0,7 & 0,7 & 0,8 \\
\hline$K L$ & Mean & 3,9 & 3,0 & 3,7 & 4,7 \\
{$[m]$} & Stdev & 1,4 & 1,2 & 1,4 & 1,8 \\
\hline$K S F$ & Mean & 6,6 & 6,2 & 10,3 & 12,5 \\
{$\left[m^{2}\right]$} & Stdev & 3,1 & 3,5 & 4,2 & 5,1 \\
\hline$K M F$ & Mean & 21,4 & 16,7 & 25,9 & 35,0 \\
{$\left[m^{2}\right]$} & Stdev & 11,3 & 10,7 & 12,7 & 16,7 \\
\hline$K V O L$ & Mean & 11,2 & 8,5 & 15,7 & 23,7 \\
{$\left[m^{3}\right]$} & Stdev & 8,9 & 8,7 & 10,9 & 15,5 \\
\hline$C R$ & Mean & 3,2 & 2,7 & 2,5 & 2,8 \\
\hline
\end{tabular}

\begin{tabular}{|c|c|rr|r|r|r|r|r|r|}
\hline & & \multicolumn{10}{|c|}{ Fichte } \\
\hline & Fl.Nr. & \multicolumn{2}{|c|}{130} & \multicolumn{2}{|c|}{131} & \multicolumn{2}{|c|}{132} & \multicolumn{2}{|c|}{133} \\
\hline & Jahr & 1992 & 1997 & 1992 & 1997 & 1992 & 1999 & 1992 & 1999 \\
\hline & $\mathrm{N}$ & 51 & 51 & 58 & 58 & 54 & 54 & 40 & 40 \\
\hline$K B$ & Mean & 4,4 & 4,1 & 4,3 & 4,3 & 4,4 & 4,0 & 4,3 & 4,1 \\
{$[m]$} & Stdev & 1,0 & 1,0 & 0,9 & 0,9 & 1,0 & 1,1 & 1,1 & 1,2 \\
\hline$K L$ & Mean & 7,0 & 4,7 & 9,9 & 6,8 & 7,3 & 6,0 & 6,3 & 6,0 \\
{$[m]$} & Stdev & 1,8 & 1,6 & 3,0 & 1,9 & 2,6 & 2,3 & 2,3 & 2,4 \\
\hline$K S F$ & Mean & 15,9 & 13,9 & 15,5 & 15,8 & 15,8 & 13,5 & 15,2 & 14,4 \\
{$\left[m^{2}\right]$} & Stdev & 7,1 & 6,3 & 6,3 & 6,6 & 7,6 & 7,0 & 7,0 & 8,2 \\
\hline$K M F$ & Mean & 52,9 & 35,3 & 71,0 & 51,2 & 55,3 & 42,8 & 47,8 & 44,5 \\
{$\left[m^{2}\right]$} & Stdev & 22,4 & 17,6 & 28,0 & 21,9 & 28,9 & 24,3 & 25,9 & 28,6 \\
\hline$K V O L$ & Mean & 39,9 & 24,6 & 53,1 & 38,4 & 42,7 & 30,7 & 35,9 & 33,6 \\
{$\left[m^{3}\right]$} & Stdev & 25,3 & 17,0 & 28,2 & 23,8 & 31,9 & 25,0 & 27,1 & 32,8 \\
\hline$C R$ & Mean & 3,3 & 2,5 & 4,6 & 3,2 & 4,1 & 3,2 & 3,1 & 3,1 \\
\hline
\end{tabular}


Deshalb entstehen größere Schattenbereiche, die zu einer Unterschätzung der Größe der Fichtenkronen führen. Akça (1989) berichtet von einer fotogrammetrischen Kronenmessung von Fichten in einer Zeitreihenanalyse. Auch hier wurden bei einer Luftbildaufnahme im September 1986 kleinere luftbildsichtbare Kronendimensionen als im Mai 1982 festgestellt.

Tab. 4.13: Ergebnisse der digitalen Bildauswertung mit Mittelwert (Mean) und Standardabweichungen (Stdev) der Kronenbreite $(K B)$, Kronenschirmfläche $(K S F)$, Kronenmantelfläche $(K M F)$ und des Kronenvolumens $(K V O L)$ von Eichen und Buchen der Versuchsfläche 502

\begin{tabular}{|c|c|rrrr|rrr|}
\hline & & \multicolumn{3}{|c|}{ Eiche } & \multicolumn{3}{c|}{ Buche } \\
\hline & Fl.-Nr. & \multicolumn{3}{|c|}{502} & \multicolumn{3}{c|}{502} \\
\hline & Jahr & 1988 & 1992 & 1999 & 1988 & 1992 & 1999 \\
\hline & $\mathrm{N}$ & 192 & 192 & 192 & 20 & 20 & 20 \\
\hline$K L$ & Mean & 4,8 & 5,9 & 8,9 & 5,3 & 5,8 & 7,3 \\
{$[m]$} & Stdev & 2,1 & 2,4 & 3,9 & 3,5 & 2,0 & 4,2 \\
\hline$K S F$ & Mean & 55,4 & 61,0 & 67,8 & 34,6 & 37,5 & 49,2 \\
{$\left[m^{2}\right]$} & Stdev & 27,5 & 30,0 & 31,9 & 26,7 & 31,5 & 38,6 \\
\hline$K M F$ & Mean & 86,0 & 117,5 & 142,9 & 60,6 & 75,6 & 112,2 \\
{$\left[m^{2}\right]$} & Stdev & 48,1 & 67,1 & 73,8 & 48,6 & 60,3 & 89,4 \\
\hline$K V O L$ & Mean & 194,7 & 250,6 & 396,0 & 121,9 & 133,6 & 271,3 \\
{$\left[m^{3}\right]$} & Stdev & 183,7 & 201,0 & 339,2 & 134,0 & 129,8 & 304,7 \\
\hline$C R$ & Mean & 1,5 & 1,9 & 2,1 & 1,8 & 2,0 & 2,3 \\
\hline
\end{tabular}

Kronenrauhigkeit $C R=K M F / K S F$

Die Kronendimensionen von Eichen und Buchen in Tab. 4.13 wurden auf Grundlage einer visuellen Kronensegmentierung, eines digitalen Oberflächenmodelles und einer weiteren Verarbeitung als Rasterdaten berechnet. Die mittleren Kronenlängen als Spannweite der Höhenwerte innerhalb eines Kronensegmentes sind größer als die visuell gemessenen Differenzen zwischen Kronenspitze und mittlerer Kronenrandhöhe (vgl. Tab. 4.10 und Tab. 4.11). Die Kronenrauhigkeit der Eichen und Buchen der digitalen Auswertung sind in der Tendenz etwas höher als die der visuellen Kartierung.

Tabelle 4.14 vergleicht Kronenlänge, -mantelfläche und -volumen der visuellen und digitalen Bildauswertung. Die Mittelwerte der Differenzen der Kronenmerkmale aus digitalem und visuellem Verfahren bestätigen die Überschätzung der Kronenlängen des automatischen Verfahrens, was zu einem entsprechend höheren mittleren Kronenvolumen führt. Die Näherung der Kronenmantelfläche mittels eines Kugelschnittes erreicht ein hohes Maß an Übereinstimmung mit der mittleren Oberfläche des digitalen Oberflä- 
chenmodelles. Das Modell eines Kugelschnittes überschätzt die mittlere Kronenoberfläche der Eichen und Buchen nur geringfügig. Im Jahr 1992 sind die Mittelwerte der Kronenmantelflächen der Eichen im Fall beider Verfahren sogar gleich. Die Standardabweichung sowie die minimalen und maximalen Werte lassen jedoch auf erhebliche Abweichungen beim Vergleich von Einzelbäumen schliessen.

Tab. 4.14: Vergleich zwischen visueller und digitaler Bildauswertung als Mittelwert (Mean), Standardabweichung (Stdev), Minimum (Min), Maximum (Max) und Anzahl (N) der Differenzen von Kronenlänge $(K L)$, Kronenmantelfläche $(K M F)$ und Kronenvolumen $(K V O L)$

\begin{tabular}{|r|rrr|rrr|rrr|}
\hline & \multicolumn{7}{|c|}{ Differenzen } \\
\hline & \multicolumn{7}{|c|}{1988} & \multicolumn{3}{c|}{1992} & & 1999 \\
\hline & \multicolumn{7}{|c|}{ Eiche N = 192 } \\
\hline & $K L$ & $K M F$ & KVOL & $K L$ & $K M F$ & $K V O L$ & $K L$ & $K M F$ & $K V O L$ \\
\hline & {$[m]$} & {$\left[m^{2}\right]$} & {$\left[m^{3}\right]$} & {$[m]$} & {$\left[m^{2}\right]$} & {$\left[m^{3}\right]$} & {$[m]$} & {$\left[m^{2}\right]$} & {$\left[m^{3}\right]$} \\
\hline Mean & 1,2 & $-12,5$ & $-58,3$ & 1,8 & 0,0 & 73,5 & 3,2 & $-10,1$ & 131,6 \\
Stdev & 1,7 & 18,2 & 126,7 & 2,0 & 32,0 & 132,6 & 3,5 & 4,3 & 258,7 \\
Min & $-1,8$ & $-91,1$ & $-104,5$ & $-0,8$ & $-70,0$ & $-181,8$ & $-2,3$ & $-242,8$ & $-607,2$ \\
Max & 11,1 & 61,7 & 1141,4 & 14,2 & 265,0 & 712,8 & 17,3 & 110,3 & 1696,6 \\
\hline
\end{tabular}

\begin{tabular}{|r|rrr|rrr|rrr|}
\hline & \multicolumn{7}{|c|}{ Differenzen } \\
\hline & \multicolumn{7}{|c|}{1988} & \multicolumn{3}{c|}{1992} & & 1999 \\
\hline & \multicolumn{7}{|c|}{ Buche N = 20 } \\
\hline & $K L$ & $K M F$ & KVOL & $K L$ & $K M F$ & KVOL & $K L$ & $K M F$ & KVOL \\
\hline & {$[m]$} & {$\left[m^{2}\right]$} & {$\left[m^{3}\right]$} & {$[m]$} & {$\left[m^{2}\right]$} & {$\left[m^{3}\right]$} & {$[m]$} & {$\left[m^{2}\right]$} & {$\left[m^{3}\right]$} \\
\hline Mean & 1,9 & $-17,4$ & 19,8 & 2,2 & $-7,3$ & 23,5 & 2,7 & $-12,7$ & 62,1 \\
Stdev & 2,8 & 18,9 & 56,2 & 2,1 & 19,3 & 71,1 & 3,1 & 3,9 & 142,8 \\
Min & $-2,0$ & $-62,3$ & $-62,3$ & $-1,0$ & $-57,0$ & $-81,8$ & $-0,1$ & $-115,8$ & $-152,8$ \\
Max & 9,4 & 10,2 & 194,0 & 6,8 & 34,1 & 203,7 & 10,7 & 41,4 & 356,4 \\
\hline
\end{tabular}

\subsubsection{Kompaktheit, Länglichkeit und Orientierung}

Die baumkronenspezifische Kompaktheit und Länglichkeit sind formbeschreibende Merkmale. Die Orientierung einer Baumkrone gibt Informationen über ihre Lage. Im Folgenden wird untersucht, ob diese Merkmale für die Trennung von Baumarten, Blattverluststufen und Baumklassen eingesetzt werden können. Die Kompaktheit ist ein In- 
dex, der in vielen vergleichenden Studien der Landschaftsökologie zum Vergleich von Flächenformen eingesetzt wird. Ein weiterer Anwendungsbereich ist der Einsatz als Kriterium für die Segmentbildung innerhalb von Fernerkundungsdaten (vgl. Baatz und Schäpe 2000). Länglichkeit und Orientierung einer Kronenschirmfläche werden aus der Näherung an eine Ellipse mit dem Flächenschwerpunkt als Zentrum bestimmt (s. Abb. 4.12).

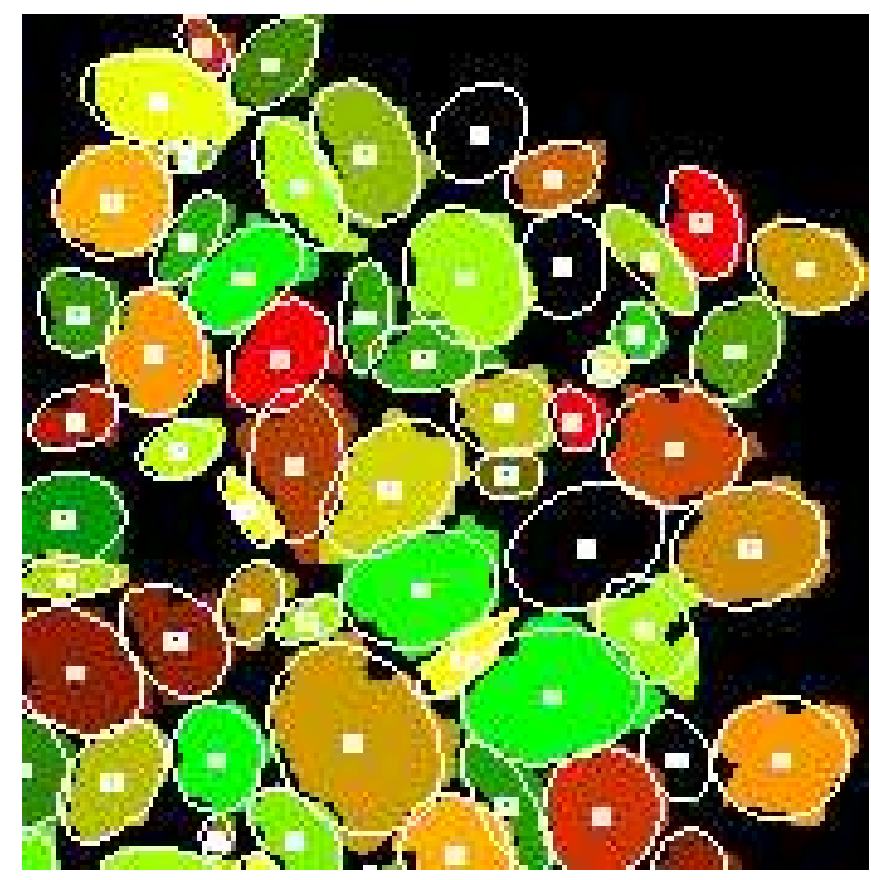

Abb. 4.12: Näherung der Kronenschirmfläche als flächengleiche Ellipse

Das Kistendiagramm ${ }^{3}$ der Abbildung 4.13 (a) läßt eine Anwendung der Kompaktheit zur Trennung von Baumarten, Blattverluststufen und Baumklassen als nicht sinnvoll erscheinen. Die Differenzen zwischen den Medianen sind nur gering und kennzeichnen eine schwache Abweichung von der Form eines Kreises $(K=1,0)$.

Die Länglichkeit als Verhältnis der Halbachsen einer an die Kronenschirmfläche genäherten Ellipse ist bei den Baumarten oder Blattverluststufen sehr ähnlich. Auffallend ist die größere Spannweite der Länglichkeit der Eichen im Vergleich zu den Buchen. Dies kann als größerer Formenreichtum der Eichenkronen im Vergleich zu den Buchenkronen interpretiert werden (Abb. 4.13 (a, b)). Werden verschiedene Luftbildbaumklassen betrachtet, so liefert zumindest die Baumklasse 3 (beherrscht) höhere Werte der Länglichkeit als die Baumklassen 1 (herrschend) und 2 (mitherrschend) (Abb. 4.13 (c)).

\footnotetext{
${ }^{3}$ Die Kistendiagramme verwenden Quartile zur Darstellung der Lage und Streuung und stellen Extremwerte speziell dar. Die Kiste wird vom unteren $(Q 1)$ bis zum oberen $(Q 3)$ Quartil eingezeichnet und beim Median unterteilt. Für die Länge des oben aufgesetzten Stabes wird die größte Beobachtung bestimmt, die kleiner als $Q 3+1,5 \cdot(Q 3-Q 1)$ ist. Die extrem großen Beobachtungen liegen oberhalb dieser angegebenen Grenze und werden als Punkte eingezeichnet.
} 


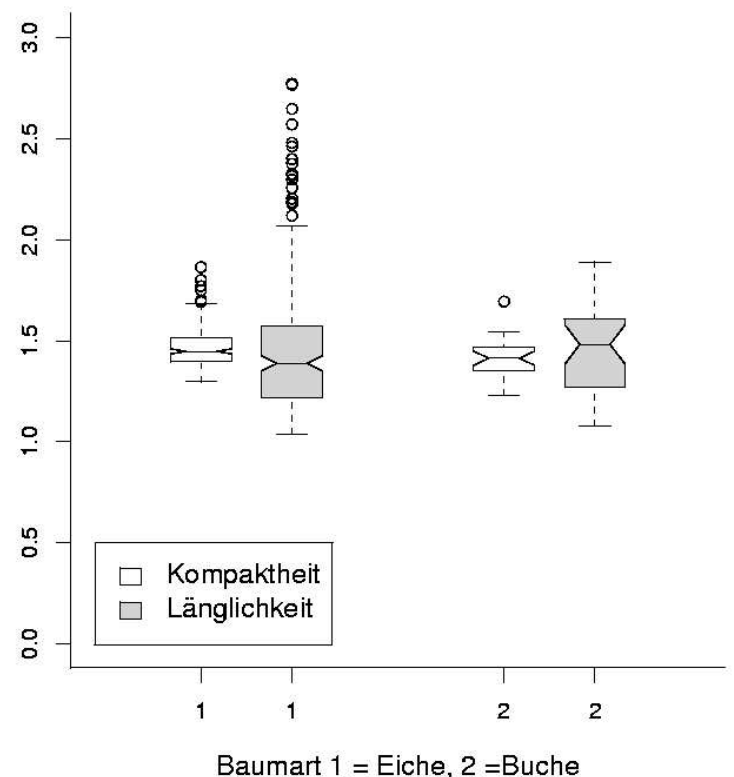

(a)

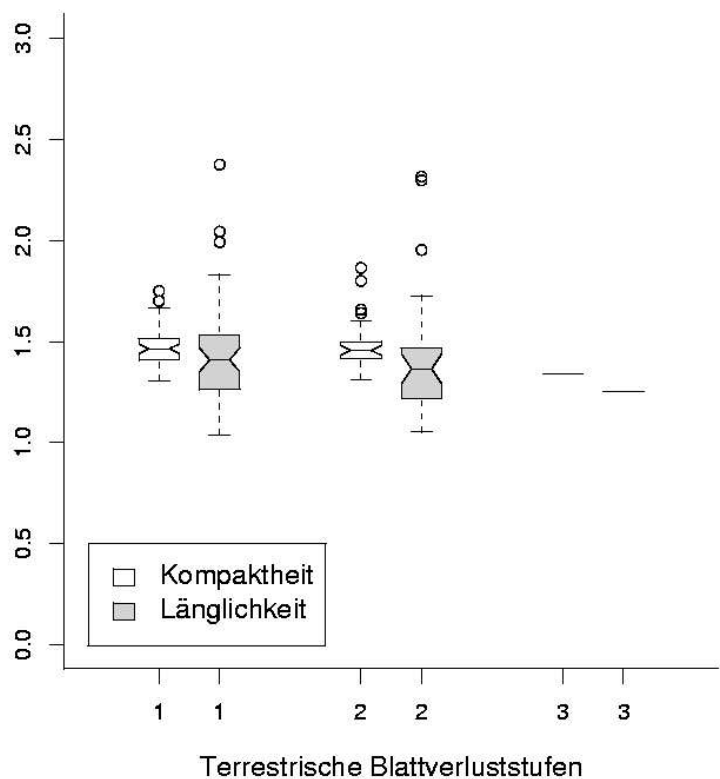

(b)

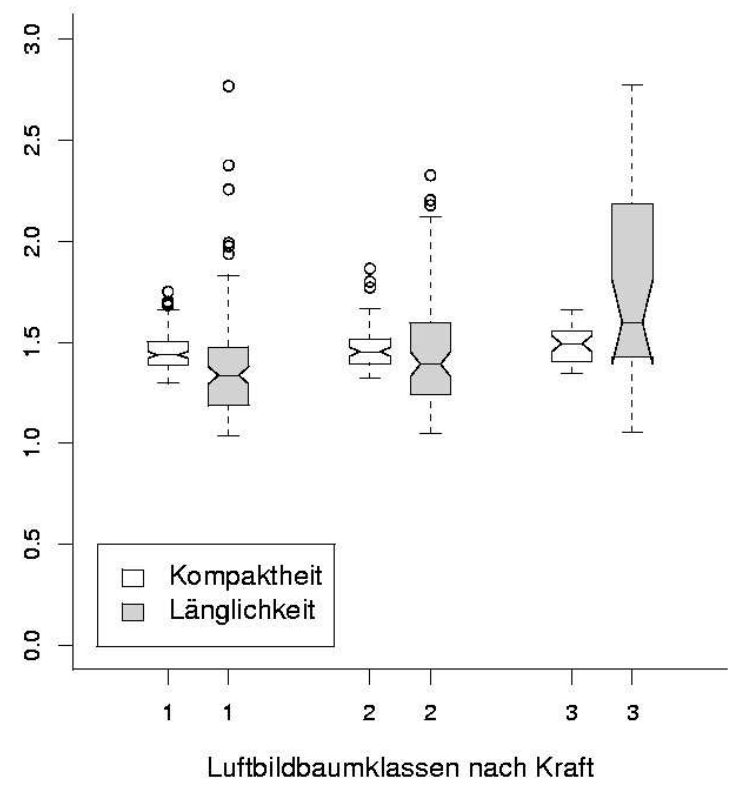

(c)

Abb. 4.13: Kompaktheit und Länglichkeit der Kronen dargestellt nach (a) Baumart, (b) Terrestrischer Blattverluststufe und (c) Luftbildbaumklasse 

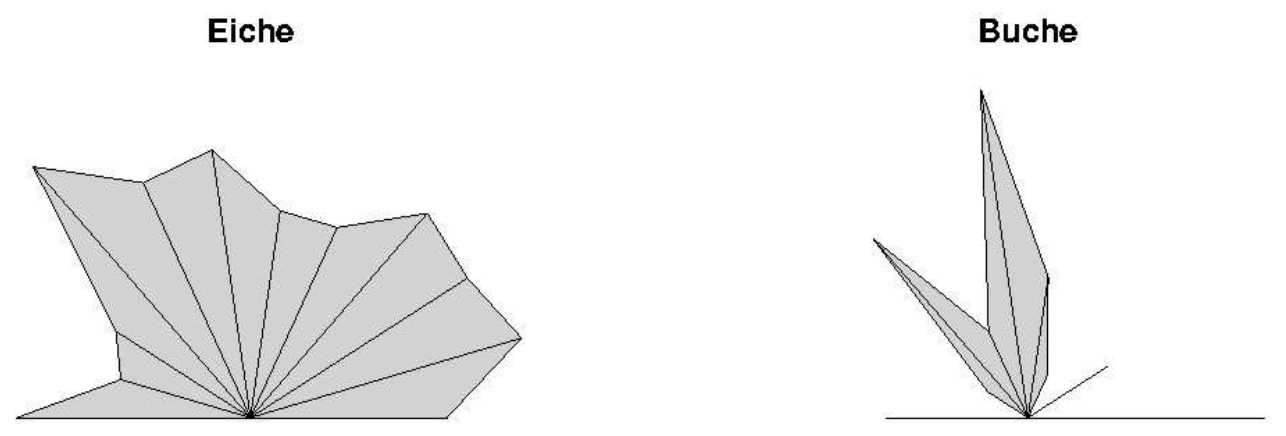

Eiche Blattverluststufe $1(0-25 \%)$

\section{Eiche Blattverluststufe 2 (26-60\%)}
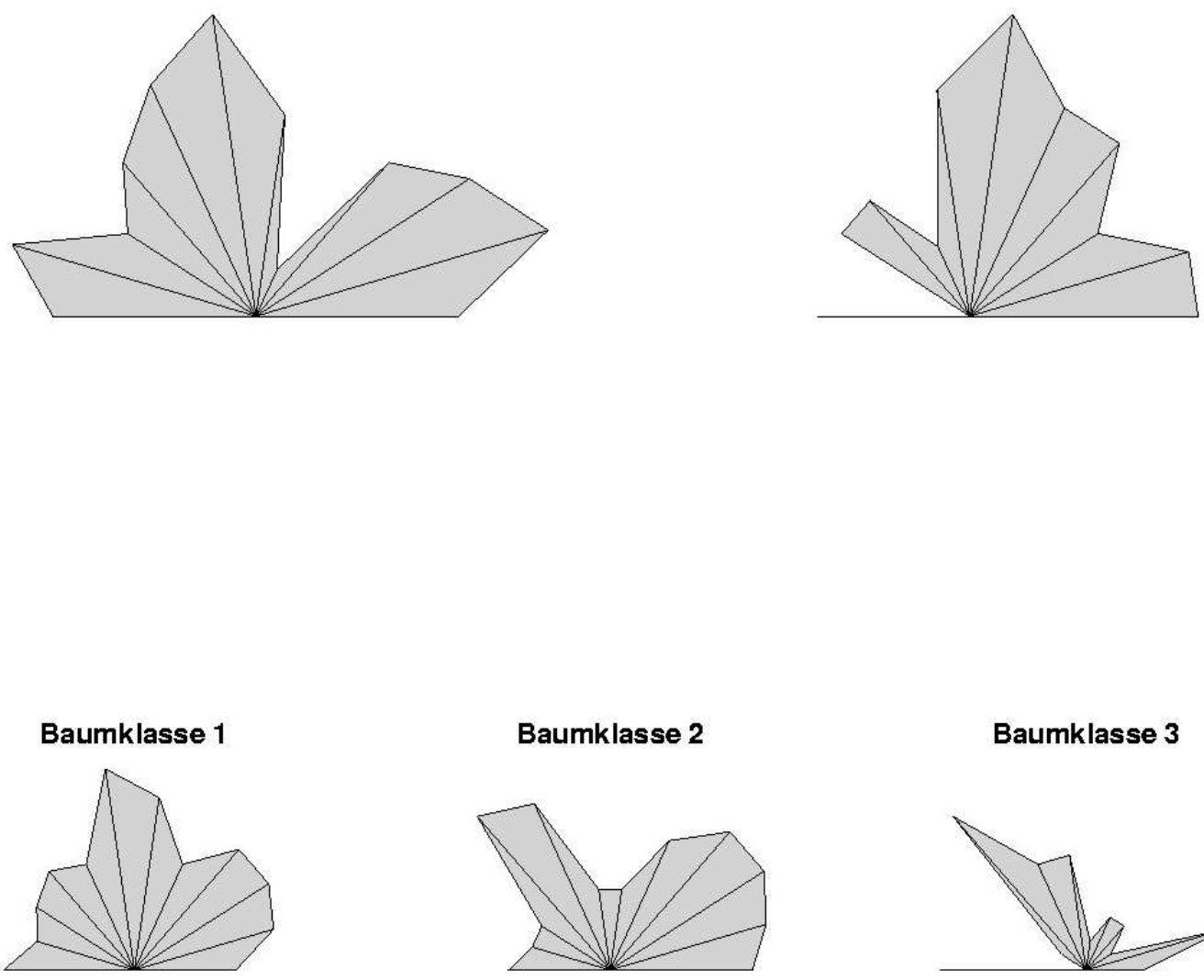

Baumklasse 2

Baumklasse 3
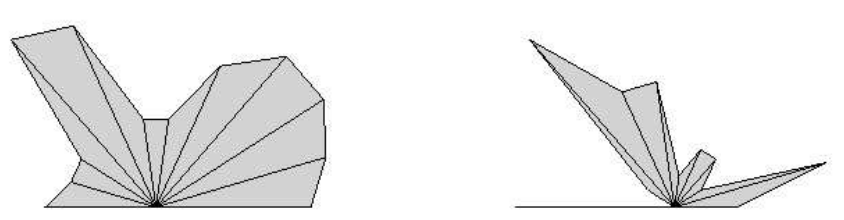

Abb. 4.14: Orientierung der Kronen nach Baumart, Blattverluststufe und Baumklasse. Dargestellt ist der Winkel zur Abszissen-Richtung $(\mathrm{O}-\mathrm{W})$ in $15^{\circ}$-Stufen, beginnend rechts mit $0^{\circ}$. 
Die Hauptorientierung der als Ellipse genäherten horizontalen Kronenschirmfläche wird als Winkel zwischen der x-Achse und der Hauptachse der Ellipse mit den möglichen Werten von $0-180^{\circ}$ definiert. Die Verteilung der Hauptorientierungen nach Baumarten, Blattverluststufen und Baumklassen veranschaulichen die Strahlendiagramme der Abbildung 4.14. Jeder Strahl repräsentiert eine Richtungsklasse (1 - 12) in einem Halbkreis mit einer Klassenbreite von $15^{\circ}$. Die Häufigkeiten werden als Distanz vom Mittelpunkt bis zum Punkt auf dem Strahl aufgetragen. Die Richtungsklassen beginnen auf der rechten Seite und nehmen links drehend zu. Fehlende Werte werden als Null behandelt.

Eichen auf der Fläche 502 haben eine relativ ausgeglichene Häufigkeitsverteilung mit einer Besetzung in allen Richtungsklassen. Die vorherrschenden Orientierungen sind $120-135^{\circ}$ und $15-30^{\circ}$. Bei den Buchen ist eine Konzentration auf wenige Richtungen festzustellen. Sie haben leicht dominierende Richtungen bei $90-105^{\circ}, 120-135^{\circ}$ und $0-15^{\circ}$. Beim Vergleich der Strahlendiagramme der Blattverluststufe 2 sind die fehlenden bzw. gering besetzten Richtungsklassen $<105^{\circ}$ auffällig. Werden die Richtungshäufigkeiten nach Baumklassen gruppiert, sind die Baumklassen 2 und 3 durch ein ausgeprägtes Maximum von $120-135^{\circ}$ gekennzeichnet. In der Baumklasse 1 sind die Richtungen von $90-105^{\circ}$ bestimmend, während diese bei den Klassen 2 und 3 unterbesetzt sind.

\subsubsection{Kronenrauhigkeit und Gaußsche Krümmung}

Wird die Rauhigkeit einer Baumkrone als Quotient aus Kronenmantelfläche und Kronenschirmfläche betrachtet, so haben Eichen eine höhere Kronenrauhigkeit als Buchen (siehe Abb. 4.15 (a)). Die Rauhigkeiten in den Blattverluststufen der Eiche sind in Klasse 1 und 2 sehr ähnlich. Mit höheren Blattverlusten (Klasse 3 und 4 (abgestorben)) verringert sie sich (s. Abb. 4.15 (b)). Die Kronenrauhigkeit stellt in dem untersuchten Bestand ein Merkmal dar, das zur Unterscheidung der Baumklassen 1 - 3 geeignet ist (Abb. 4.15 (c)).

Als weiteres Merkmal zur Charakterisierung der Kronenoberflächen wurde der Absolutwert der Gaußschen Krümmung berechnet. Die Mittelwerte geben Auskunft über die durchschnittliche Oberflächenkrümmung in einem Fenster von $5 \times 5$ Bildelementen. Das entspricht einem Quadrat der Seitenlänge von 1,25 m der natürlichen Kronenoberfläche. Eichen haben in dieser Auflösung geringere Oberflächenkrümmungen als $\mathrm{Bu}-$ chen, was vermutlich mit dem typischen Kronenaufbau mit steil aufragenden Ästen der Buchen im Gegensatz zu den kompakten Ballenstrukturen der Eichen zusammenhängt. Zur Unterscheidung der Blattverluststufen 1 und 2 der Eichen und der Baumklassen ist dieses Merkmal weniger geeignet, wie aus Abb. 4.15 ersichtlich ist. 


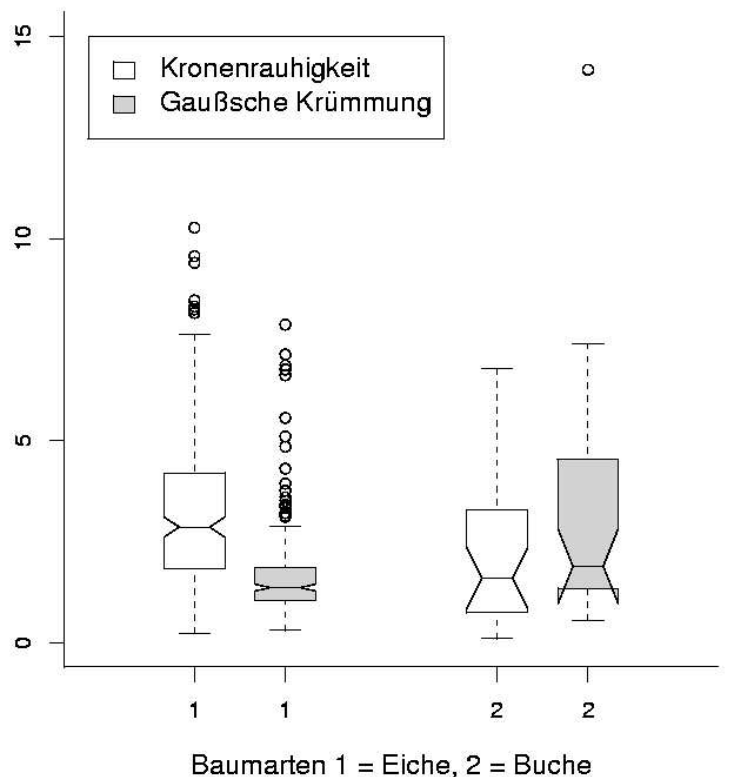

(a)

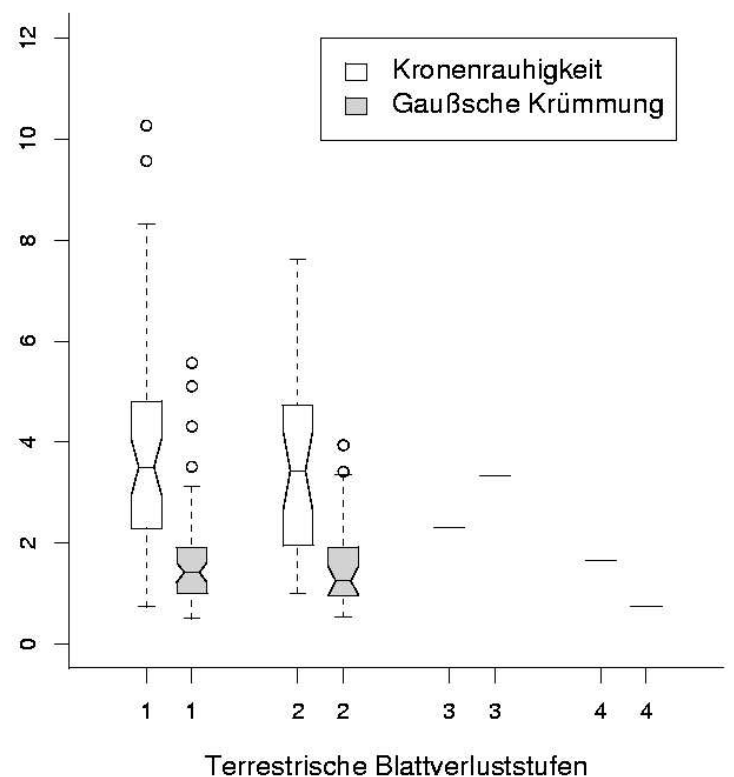

(b)

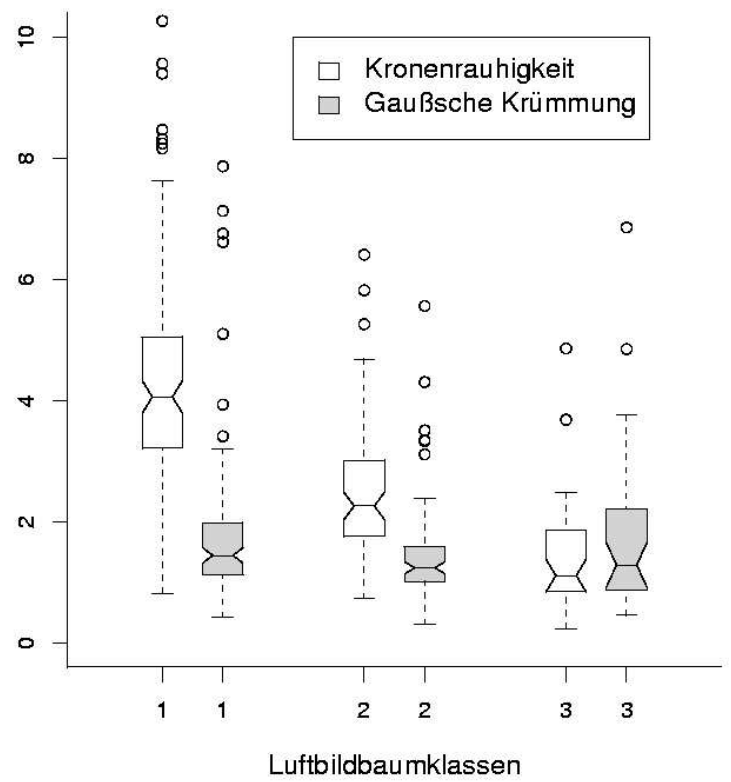

(c)

Abb. 4.15: Rauhigkeit und Gaußsche Krümmung der Kronen dargestellt nach (a) Baumart, (b) Terrestrische Blattverluststufe und (c) Luftbildbaumklasse 


\subsection{Erkennung von ausgeschiedenen Bäumen}

Die NDVI-Kanäle der Orthofotos aus den Befliegungsjahren 1993, 1994, 1995 wurden für die Erkennung von Bäumen verwendet, die im Zuge einer Durchforstung auf der Fläche 502 entnommen wurden oder abgestorben sind. In einer multitemporalen Zeitreihenanalyse mit drei Bildaufnahmen aus verschieden Jahren können die primären und komplementären Farben, die auf dem Bildschirm entstehen, hinsichtlich einer Veränderung interpretiert werden. Das Datum der Aufnahme und die Zuordnung zum Farbkanal müssen bekannt sein (Blau: 1993, Rot: 1994, Grün: 1995). Ein RGB-ClusterVerfahren reduziert die ursprünglichen $256^{3}$ Farben auf nur noch 27 Farben. Die Farbkomposite der Abbildung 4.16 wird visuell interpretiert:

Tab. 4.15: Interpretation der additiven Farben in der RGB-Farbkomposite (vgl. Sader und Winne 1992)

\begin{tabular}{ccccc}
\hline & \multicolumn{3}{c}{ NDVI-Datum } & \\
\cline { 2 - 4 } Additive Farbe & 1993 & 1994 & 1995 & Interpretation der Änderung \\
\hline Schwarz & G & G & G & geringer Biomassenanteil, keine Änderung \\
Blau & H & G & G & Abnahme 1993 - 1994 \\
Rot & G & H & G & Anstieg 1993 bis 1994, Abnahme 1994 - 1995 \\
Grün & G & G & H & Anstieg1993 bis 1994 \\
Purpur & H & H & G & Abnahme 1994 - 1995 \\
Blaugrün & H & G & H & Abnahme 1993 - 94, Zunahme 1994 - 1995 \\
Gelb & G & H & H & Anstieg 1993 - 1994 - 1995 \\
Weiß, Hellgrau & H & H & H & Hoher Biomassenanteil, keine Änderung \\
\hline
\end{tabular}

$\mathrm{G}=$ Geringer Anteil grüner Biomasse, $\mathrm{H}=$ Hoher Anteil grüner Biomasse

Auffallend ist ein blaugrünes Band im oberen Drittel des Bildausschnittes (Abb. 4.16 (a)). Diese Farbe kommt durch eine Kombination eines hohen Blau-, geringen Rotund hohen Grün-Anteiles zustande. Die visuelle Überprüfung ergab eine auffallende Häufung von chlorotischen Baumkronen im Jahr 1994, die sich 1995 wieder regeneriert haben.

Aus den 27 Farbklassen wurden 3 Klassen mit blauen Farbtönen selektiert. Alle Bildpunkte der Farbe Blau mit einer Flächengröße unter $6 \mathrm{~m}^{2}$ (entspricht einer Mindestfläche der visuellen Kronenkartierung) wurden nicht weiter berücksichtigt. Die verbleibenden Regionen sind Baumkronen, die im Zeitraum 1993 - 1994 im Wege einer Durchforstung ausgeschieden sind.

Abbildung 4.16 (b) zeigt das Ergebnis eines Vergleiches zwischen Luftbildinterpretation und digitaler Klassifikation der NDVI-Farbkomposite. 


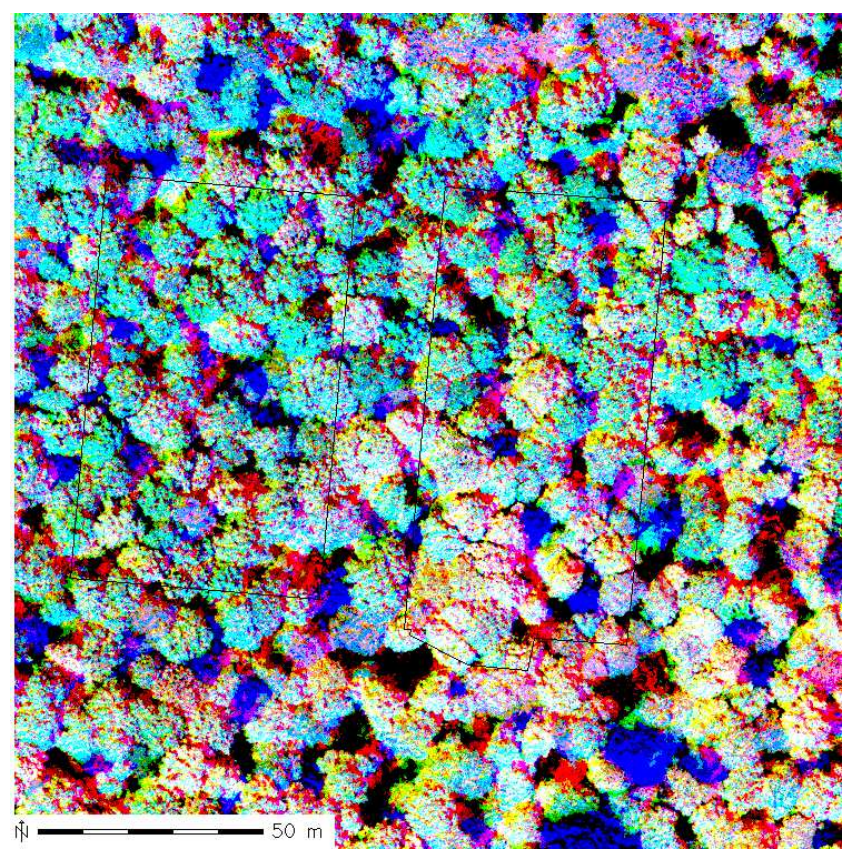

(a) Multitemporale Farbkomposite aus 3 NDVIKanälen: 1993 (blau), 1994 (grün), 1995 (rot)

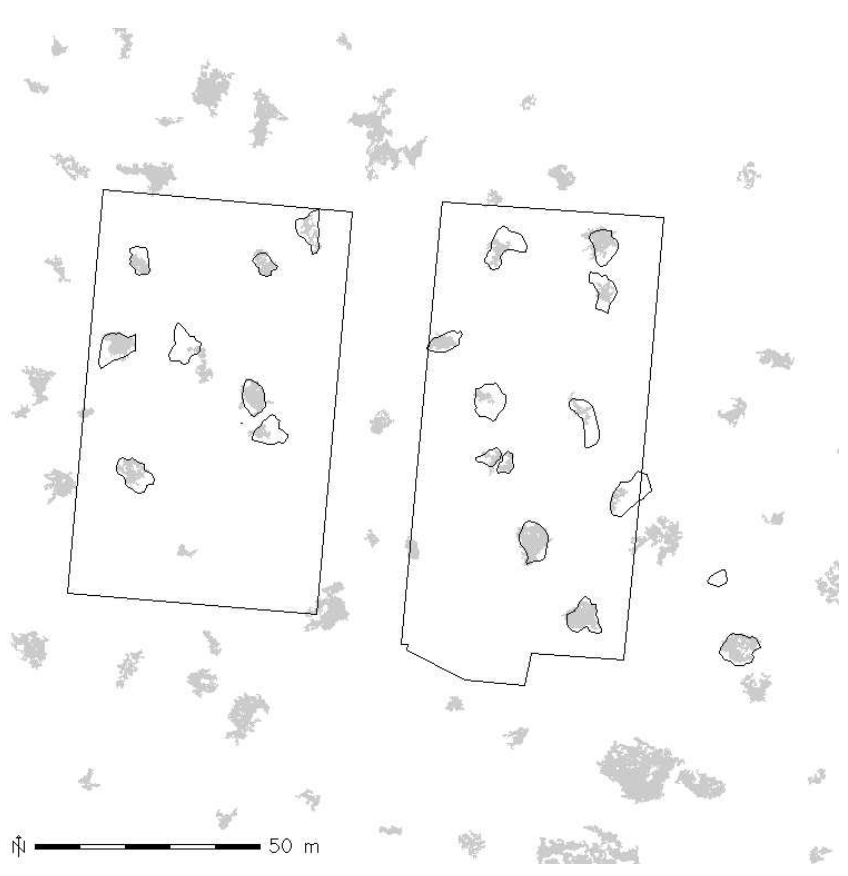

(b) Binärbild der entnommenen Bäume 1993-1994 als Ergebnis der RGB-Farbkomposite überlagert mit Vektoren als Ergebnis einer visuellen Luftbildinterpretation

Abb. 4.16: Erkennung von ausgeschiedenen Bäumen anhand einer multitemporalen RGB-NDVI-Farbkomposite 
Die visuelle Interpretation ergab als Referenz 19 Bäume, die im Zeitraum 1993 bis 1994 auf den Teilflächen BDF und LEVEL2 (502) entnommen wurden. Die Vektoren der Kronenschirmflächen dieser identifizierten Bäume überlagern das Binärbild der automatischen Erkennung der ausgeschiedenen Bäume mittels der multitemporalen NDVI-Farbkomposite. Mit dem halbautomatischen Verfahren wurden 23 entnommene Bäume identifiziert. Eine visuelle Überprüfung ergab eine gute Übereinstimmung mit den Ergebnissen der Luftbildinterpretation. In zwei Fällen wurde die Entnahme von Randbäumen bei der visuellen Auswertung der Untersuchungsflächen übersehen. Zwei Bäume der Klassenbildung der NDVI-Farbkomposite konnten nicht als Entnahme bestätigt werden.

Die Methode ist geeignet, die Anzahl der ausgeschiedenen Bäume nach einer Durchforstung zu schätzen. Das Intervall zwischen den Befliegungszeitpunkten darf hierbei nicht zu groß werden. Der Zeitraum zwischen den Befliegungen sollte fünf Jahre möglichst nicht überschreiten, da sich die entstandenen Bestandeslücken wieder schließen und ein Nachweis der entnommenen Einzelbäume schwieriger wird. Als Voraussetzung sollten die Luftbilder möglichst zur gleichen Jahres- und Tageszeit aufgenommen werden, um Unterschiede, die auf phänologische Veränderungen zurückgehen, auszuschließen. Schwankungen der Farbwiedergabe, die aufgrund von Unterschieden in der Sensitivivtät der Filmemulsionen und des Entwicklungsprozesses entstehen, sollten möglichst gering sein. Eine hohe geometrische Genauigkeit der Luftbildentzerrung auf Grundlage signalisierter Passpunkte ist zu empfehlen.

\subsection{Baumart}

Die Baumarten Eiche und Buche wurden terrestrisch bestimmt und den visuell segmentierten Baumkronen als Attribut zugeordnet. Als Trainingsdaten für die Baumartenerkennung wurden 75 Bäume (68 Eichen und 7 Buchen) zufällig ausgewählt, als TestDaten zur Überprüfung der Diskriminanzfunktion wurden 183 Bäume klassifiziert. Für die Test-Daten wurden a priori gleiche relative Häufigkeiten der Baumarten angenommen.

Die folgende Tabelle gibt die Ergebnisse der schrittweisen Vorwärtsauswahl der am besten geeigneten Variablen wieder. Die baumkronenspezifische Gaußsche Oberflächenkrümmung ist offensichtlich sehr gut geeignet, Eichen und Buchen zu trennen. Die Gesamtgenauigkeit steigt unter Verwendung der Kronenrauhigkeit und des Texturmerkmales contrast auf $88 \%$ an. Die höchste Gesamtgenauigkeit von $89 \%$ wird mit vier räumlichen Variablen und einer spektralen Variablen (zweite Hauptkomponente) erreicht. Bei Verwendung von mehr als 5 Variablen verringert sich die Gesamtgenauigkeit. Spektrale Merkmale haben nach diesen Ergebnissen eine geringere Bedeutung als Gestaltmerkmale, was mit den Interpretationsanweisungen zur Unterscheidung von Laubholzarten übereinstimmt. Die erreichte Genauigkeit ist vergleichbar mit Ergebnissen einer visuellen Luftbildinterpretation. 
Tab. 4.16: Ergebnisse der linearen Diskriminanzanalyse zur Trennung der Baumarten Eiche und Buche

\begin{tabular}{cccccc}
\hline Anz. & $\begin{array}{c}\text { Anz. } \\
\text { Unters. }\end{array}$ & Beste Teilmenge & OA & $\kappa$ & S.E. \\
\hline 1 & 8 & gc & 0,86 & 0,34 & 0,0074 \\
2 & 7 & kr, gc & 0,87 & 0,36 & 0,0074 \\
3 & 6 & con, kr, gc & 0,88 & 0,38 & 0,0073 \\
4 & 5 & k, con, kr, gc & 0,85 & 0,42 & 0,0074 \\
5 & 4 & pc , k, con, gc & 0,89 & 0,46 & 0,0740 \\
6 & 3 & var, pc , k, con, cr, gc & 0,89 & 0,42 & 0,0737 \\
7 & 2 & lang, var, pc , k, con, kr, gc & 0,89 & 0,40 & 0,0073 \\
8 & 1 & ndvi, lang, var, pc, k, con, kr, gc & 0,85 & 0,29 & 0,0739 \\
\hline
\end{tabular}

Gesamtgenauigkeit (OA), Kappa-Maß (kappa), Standardfehler (S.E.), pc = 2. Hauptkomponente, $\mathrm{kr}=$ Kronenrauhigkeit, $\mathrm{gc}=$ Absolutwert der Gaußschen Krümmung, con $=$ Texturma $ß$ contrast, var $=$ Texturma $\$$ variance, ndvi $=$ Vegetationsindex, $\mathrm{k}=$ Kompaktheit, lang = Länglichkeit

\subsection{Vitalität}

Als Referenz für die Unterscheidung von relativen Kronenverlichtungsstufen wurden für die Blattverluststufe $1(0$ - $25 \%) 22$ Eichen verwendet. Für die Blattverluststufe 2 (26- $60 \%$ ) wurden 18 Eichen zufällig ausgewählt. Als Testbäume standen 39 Bäume zur Verfügung, deren Verlichtungsgrad im gleichen Jahr im Gelände beobachtet wurde. Wird nur eine Variable für die Bestimmung der Diskriminanzfunktion ausgewählt, so ist die zweite Hauptkomponente mit $74 \%$ Gesamtgenauigkeit am besten geeignet. Diese Trefferquote lässt sich nur noch durch die zusätzliche Auswahl der Kronenrauhigkeit und der Gaußschen Krümmung auf 80 \% erhöhen. Die Texturmerkmale contrast und variance und der Vegetationsindex NDVI tragen nicht zu einer zusätzlichen Verbesserung bei. Dennoch sind die spektralen Informationen der 2. Hauptkomponente von größerer Bedeutung für die Einschätzung der Kronenverlichtung als Formmerkmale. Die Testergebnisse der visuellen Interpretation von 4 Blattverluststufen durch einen erfahrenen Luftbildinterpreten lagen unter Verwendung der gleichen Trainingsund Testbäume bei Gesamtgenauigkeiten von 51 - 67 \% (Akça et al. 2002). 
Tab. 4.17: Ergebnisse der linearen Diskriminanzanalyse zur Trennung der Blattverluststufen 1 und 2

\begin{tabular}{cccccc}
\hline $\begin{array}{c}\text { Subset } \\
\text { Größe }\end{array}$ & Anz. & Beste Teilmenge & OA & $\kappa$ & S.E. \\
\hline 1 & 6 & pc & 0,74 & 0,41 & 0,1556 \\
2 & 5 & kr, pc & 0,74 & 0,44 & 0,1508 \\
3 & 4 & gc, kr, pc & 0,80 & 0,52 & 0,1555 \\
4 & 3 & con, gc, kr, pc & 0,74 & 0,47 & 0,1450 \\
5 & 2 & ndvi, con, gc, kr, pc & 0,72 & 0,43 & 0,1420 \\
6 & 1 & var, ndvi, con, gc, kr, pc & 0,74 & 0,44 & 0,1510 \\
\hline
\end{tabular}

Gesamtgenauigkeit (OA), Kappa-Maß (kappa), Standardfehler (S.E.), pc = 2. Hauptkomponente, $\mathrm{kr}=$ Kronenrauhigkeit, $\mathrm{gc}=$ Absolutwert der Gaußschen Krümmung, con $=$ Texturma $ß$ contrast, var $=$ Texturma $ß$ variance, ndvi $=$ Vegetationsindex

\subsection{Beziehungen zwischen Grundflächenzuwachs und Kronenvariablen}

Die Beziehungen zwischen den im Luftbild gemessenen Kronenvariablen und dem terrestrisch hergeleiteten Grundflächenzuwachs wurden am Beispiel von Eichen, Buchen und Fichten untersucht. Als Variablen wurden die Kronenbreite, -länge, -schirmfläche, -mantelfläche und -volumen einer visuellen Luftbildauswertung eingesetzt. Im Fall der Eichen von Fläche 502 wurden die Kronengrößen zusätzlich mit dem digitalen Auswertungsverfahren hergeleitet (vgl. Kap. 3.3.3.4 ). Als zusätzliche Variable wurde der Belaubungsgrad (BG) berücksichtigt als

$$
B G=(100-B L V) / 100
$$

wobei $B L V$ das Nadel-/Blattverlustprozent ist, das am Anfang oder Ende des Untersuchungszeitraumes visuell in 5\%-Stufen im Luftbild geschätzt wurde. Es wurden drei verschiedene Regressionsmodelle analysiert, um festzustellen, ob sich die im Luftbild geschätzten Kronenvariablen zu einer Schätzung des Grundflächenzuwachses eignen. Zunächst wurden die Beziehungen der Kronendimensionen zum Grundflächenzuwachs in univariaten linearen Regressionen analysiert. In einem weiteren univariaten linearen Modell wurde das Produkt aus den Kronendimensionen mit dem Belaubungsgrad gebildet, um zu untersuchen, ob die Gewichtung der Kronengrößen mit den baumspezifischen Belaubungsgraden zur Verbesserung der Schätzung des Grundflächenzuwachses beitragen. Dem wurde ein multiples lineares Modell mit den unabhängigen Variablen einer Kronendimension und dem Belaubungsgrad gegenübergestellt.

In Tabelle 4.18 werden die statistischen Kennwerte des Grundflächen- und Durch- 
messerzuwachses dargestellt. Die untersuchten Eichen der Fläche 405 weisen die geringsten mittleren Durchmesser- und Grundflächenzuwächse auf. Dies ist auf das Alter von über 200 Jahren und auf das Bodensubstrat des Mittleren Buntsandsteins zurückzuführen. Die Fichten der Flächen 130-133 stocken ebenfalls auf Sandsteinsubstraten und sind im Vergleich zu den Fichten der Fläche 108 geringwüchsig (Tab. 4.18).

Tab. 4.18: Mittelwert $(\bar{x})$, Standardabweichung ( $s$, Minimum (Min) und Maximum (Max) des Durchmesser- $\left(I_{d}\right)$ und Grundflächenzuwachses $\left(I_{g}\right)$. Ei $=$ Eiche, $\mathrm{Bu}=$ Buche, $\mathrm{Fi}=$ Fichte

\begin{tabular}{ccc|ccccc|rrrr}
\hline & & & \multicolumn{5}{|c|}{$I_{d}[\mathrm{~mm}]$} & \multicolumn{4}{|c}{$I_{g}\left[\mathrm{~cm}^{2}\right]$} \\
Art & $\mathrm{N}$ & Fl.Nr. & Periode & $\bar{x}$ & $s$ & Min & Max & $\bar{x}$ & $s$ & Min & Max \\
\hline $\mathrm{Ei}$ & 72 & 502 & $92-99$ & 27,2 & 9,9 & 8,0 & 55,0 & 223,3 & 107,7 & 56,2 & 569,0 \\
$\mathrm{Ei}$ & 45 & 405 & $92-99$ & 12,8 & 5,1 & 2,2 & 23,7 & 87,8 & 46,3 & 9,2 & 199,6 \\
$\mathrm{Bu}$ & 30 & 203 & $86-94$ & 26,6 & 16,2 & 0,0 & 72,0 & 229,7 & 152,0 & 0,0 & 725,6 \\
$\mathrm{Fi}$ & 100 & 108 & $86-94$ & 23,9 & 9,1 & 3,5 & 50,5 & 113,3 & 52,4 & 11,0 & 251,3 \\
$\mathrm{Fi}$ & 175 & $130-33$ & $94-99$ & 8,3 & 5,4 & 0,0 & 36,3 & 83,3 & 53,61 & 0,4 & 362,9 \\
\hline
\end{tabular}

Die Ergebnisse der linearen einfachen Regressionen werden in den Tabellen 4.19, 4.20, 4.21 dargestellt.

Die logarithmische Transformation der Kronendimensionen ergab nur in wenigen Fällen eine bessere Anpassung der Modelle, so dass sich die nachfolgenden Betrachtungen auf die untransformierten Modelle beschränken. Voraussetzungen der Regressionsanalyse sind ein linearer Zusammenhang zwischen den genannten Größen und normal verteilte, unabhängige Residuen mit gleichmäßiger Streuung. Eine Abweichung von dieser Annahme wurde in Streudiagrammen der Residuen gegen die angepassten Werte festgestellt: mit zunehmenden Wert beispielsweise der Zielvariablen Kronenvolumen und -mantelfläche nimmt die Streuung leicht zu.

Die Kronendimensionen der Baumart Eiche (Flächen 502 und 405) zeigen die höchsten Bestimmtheitsmaße zur Schätzung des Grundflächenzuwachses im Vergleich zu den Baumarten Fichte und Buche (Tab. 4.19). Kronenmantel- und Kronenschirmfläche am Ende des Untersuchungszeitraumes sind die besten erklärenden Variablen. Die Verwendung von Kronenmantelflächen, die aus visuell abgegrenzten Segmenten und digitalen Oberflächenmodellen berechnet wurden, (s. digitale Auswertung, Kap. 3.3.4.2) verbessert die Regressionsmodelle der Eichen auf Fläche 502 zum Zeitpunkt 1999 (s. Tab. 4.19). $71 \%$ der Variation des Grundflächenzuwaches wird in diesem Modell durch die Kronenmantelfläche erklärt. Im Jahr 1992 sind keine Unterschiede zwischen der Herleitung der Kronenmantelfläche als Oberfläche eines Kugelschnittes (KMF92) und der Verwendung des automatisch generierten digitalen Oberflächenmodelles (AKMF92) zu erkennen. Die Berechnung der Lichtkronenlänge aus der Spannweite der Höhen innerhalb eines Kronensegmentes führt zu weniger straffen Zusammenhängen mit dem Grundflächenzuwachs als die Herleitung der Kronenlänge aus der Differenz zwischen Höhe der Kronenspitze und mittlerer Höhe der Kronenrandpunkte (vgl. Kap. 3.3.3.4). 
Tab. 4.19: Ergebnisse der einfachen linearen Regressionsanalyse der Kronenvariablen der Eichen gegen den Grundflächenzuwachs (GZ) 1992 -1999. Visuelle Bildauswertung: Kronenbreite $(K B)$, Kronenlänge $(K L)$, Kronenschirmfläche $(K S F)$, Kronenmantelfläche $(K M F)$, Kronenvolumen $(K V O L)$; Digitale Bildauswertung: automatische Kronenbreite $(A K B)$, automatische Kronenmantelfläche $(A K M F)$, automatisches Kronenvolumen $(A K V O L)$

\begin{tabular}{|c|c|c|c|c|c|c|}
\hline \multicolumn{7}{|c|}{ Eiche $\mathrm{N}=72$ (Fläche 502) } \\
\hline $\mathrm{y}$ & $\mathrm{x}$ & $\beta_{0}$ & $\beta_{1}$ & $s e_{\beta_{1}}$ & $\sqrt{M S_{R e s}}$ & $R^{2}$ \\
\hline \multirow[t]{16}{*}{ GZ9299 } & AKMF99 & 50,789 & 1,209 & 0,099 & 58,50 & 0,709 \\
\hline & KSF99 & 43,687 & 2,596 & 0,244 & 67,00 & 0,618 \\
\hline & KMF99 & 66,908 & 1,040 & 0,112 & 72.50 & 0,553 \\
\hline & KVOL99 & 118,541 & 0,404 & 0,044 & 72,90 & 0,548 \\
\hline & KB99 & $-88,043$ & 34,196 & 3,721 & 73,00 & 0,547 \\
\hline & KMF92 & 37,827 & 1,591 & 0,180 & 74,50 & 0,529 \\
\hline & KVOL92 & 100,648 & 0,704 & 1,081 & 75,10 & 0,520 \\
\hline & AKMF92 & 94,750 & 1,031 & 0,122 & 76,20 & 0,506 \\
\hline & KSF92 & 59,883 & 2,628 & 0,315 & 76,80 & 0,499 \\
\hline & KB92 & $-89,681$ & 36,152 & 4,386 & 77,20 & 0,493 \\
\hline & AKVOL99 & 148,520 & 0,188 & 0,026 & 81,98 & 0,428 \\
\hline & KL99 & 34,120 & 39,31 & 6,260 & 86,70 & 0,360 \\
\hline & KL92 & $-12,620$ & 58,50 & 10,03 & 89,00 & 0,327 \\
\hline & AKVOL92 & 154,864 & 0,254 & 0,047 & 91,11 & 0,294 \\
\hline & AKL99 & 141,578 & 10,094 & 2,744 & 99,25 & 0,162 \\
\hline & AKL92 & 149,688 & 11,675 & 3,868 & 102,0 & 0,115 \\
\hline \multicolumn{7}{|c|}{ Eiche $\mathrm{N}=45$ (Fläche 405$)$} \\
\hline \multirow[t]{9}{*}{ GZ9299 } & KB99 & $-61,671$ & 21,716 & 2,304 & 26,73 & 0,674 \\
\hline & KSF99 & 11,411 & 1,928 & 0,202 & 26,50 & 0,679 \\
\hline & KVOL92 & 25,892 & 0,442 & 0,053 & 29,06 & 0,615 \\
\hline & KB92 & $-74,996$ & 23,202 & 2,913 & 29,75 & 0,596 \\
\hline & KMF92 & 2,467 & 0,841 & 0,106 & 29,77 & 0,596 \\
\hline & KL92 & $-14,611$ & 24,187 & 4,731 & 36,92 & 0,378 \\
\hline & KVOL99 & 61,582 & 0,1339 & 0,043 & 42,19 & 0,188 \\
\hline & KMF99 & 51,536 & 0,2809 & 0,092 & 42,41 & 0,179 \\
\hline & KL99 & 47,018 & 8,042 & 4,011 & 44,76 & 0,086 \\
\hline
\end{tabular}

$y=$ Zielvariable, $x=$ Regressor, $\beta_{0}=$ Achsenabschnitt, $\beta_{1}=$ Steigung, $s_{\beta_{1}}=$ Standardabweichung der Steigung, $\sqrt{M S_{\text {Res }}}=$ Standardabweichung der Residuen, $R^{2}=$ Bestimmtheitsmaß 
Die berechneten Bestimmtheitsmaße der Buchen der univariaten linearen Modelle sind sehr gering (Tab. 4.20). Maximal 33 - $34 \%$ der Variation des Grundflächenzuwachses können durch das Kronenvolumen bzw. die Kronenmantelfläche am Anfang des Untersuchungszeitraumes erklärt werden. Die Kronendimensionen zum Beginn des Zeitraumes 1995 sind nicht für eine Schätzung des Grundflächenzuwachses geeignet. Ursachen liegen vermutlich in der geringen Variation der Kronendimensionen auf Fläche 203 (vgl. Tab. 4.11) und in Fehlern, die bei der Abgrenzung der Buchenkronen in einem dicht geschlossenen Kronendach entstehen. Eine Trennung einzelner Kronen ist auch mit Hilfe einer stereoskopischen Bildauswertung am analytischen Stereoplotter nicht immer eindeutig möglich.

Die Zusammenhänge zwischen Luftbildkronengrößen und Grundflächenzuwachs sind auf den Flächen 130 - 133 weniger straff (Tab. 4.21).

Kronenmantelfläche, -schirmfläche und -breite am Ende des Untersuchungszeitraumes sind in den besten Modellen vertreten und erklären 49 - 51\% der Variation des Grundflächenzuwachses auf Fläche 108. Die linearen Modelle mit Verwendung der Kronenlängen der Fichten sind am wenigsten geeignet für eine Schätzung des Grundflächenzuwachses.

Tab. 4.20: Ergebnisse der einfachen linearen Regressionsanalyse der Kronenvariablen der Buchen gegen den Grundflächenzuwachs (GZ) 1986-1994. Visuelle Bildauswertung: Kronenbreite $(K B)$, Kronenlänge $(K L)$, Kronenschirmfläche $(K S F)$, Kronenmantelfläche $(K M F)$, Kronenvolumen $(K V O L)$

\begin{tabular}{ccrrrrr}
\hline \multicolumn{7}{c}{ Buche N = 30 (Fläche 203) } \\
\hline$y$ & $\beta_{0}$ & $\beta_{1}$ & $s e_{\beta_{1}}$ & $\sqrt{M S_{\text {Res }}}$ & $R^{2}$ \\
\hline GZ8694 & KVOL87 & 52,700 & 1,424 & 0,374 & 125,6 & 0,341 \\
& KMF87 & -46.775 & 2,717 & 0,735 & 126,8 & 0,328 \\
& KSF87 & 15,426 & 3,482 & 3,104 & 133,4 & 0,256 \\
& KB87 & -226.80 & 47.90 & 14.720 & 131,7 & 0,274 \\
& KSF95 & 56,367 & 2,225 & 1,040 & 143,4 & 0,140 \\
KB95 & $-73,03$ & 28,03 & 14,760 & 145,6 & 0,114 \\
& KL87 & 61,87 & 57,86 & 1,640 & 147,7 & 0,088 \\
& KL95 & 237,639 & $-1,779$ & 19,080 & 154,6 & 0,000 \\
KMF95 & 171,524 & 0,354 & 0,456 & 153,0 & 0,021 \\
KVOL95 & 198,595 & 0,112 & 0,175 & 153,8 & 0,014 \\
\hline
\end{tabular}

$y=$ Zielvariable, $x=$ Regressor, $\beta_{0}=$ Achsenabschnitt, $\beta_{1}=$ Steigung, $s_{\beta_{1}}=$ Standardabweichung der Steigung, $\sqrt{M S_{\text {Res }}}=$ Standardabweichung der Residuen, $R^{2}=$ Bestimmtheitsmaß 
Tab. 4.21: Ergebnisse der einfachen linearen Regressionsanalyse der Kronenvariablen der Fichten gegen den Grundflächenzuwachs (GZ) 1986 -1994 bzw. 1994 - 1999. Visuelle Bildauswertung: Kronenbreite $(K B)$, Kronenlänge $(K L)$, Kronenschirmfläche $(K S F)$, Kronenmantelfläche $(K M F)$, Kronenvolumen $($ KVOL)

\begin{tabular}{|c|c|c|c|c|c|c|}
\hline \multicolumn{7}{|c|}{ Fichte $\mathrm{N}=100$ (Fläche 108) } \\
\hline$y$ & $x$ & $\beta_{0}$ & $\beta_{1}$ & $s e_{\beta_{1}}$ & $\sqrt{M S_{R e s}}$ & $R^{2}$ \\
\hline \multirow[t]{10}{*}{ GZ8694 } & KSF95 & 15,749 & 7,663 & 0,758 & 37,06 & 0,510 \\
\hline & KMF95 & 31,019 & 2,297 & 0,234 & 37,61 & 0,496 \\
\hline & KB95 & $-79,956$ & 46,630 & 4,764 & 37,66 & 0,494 \\
\hline & KVOL95 & 54,112 & 2,430 & 0,248 & 37,67 & 0,494 \\
\hline & KB86 & $-46,094$ & 52,477 & 5,242 & 37,24 & 0,506 \\
\hline & KSF86 & 37,669 & 11,660 & 1,265 & 38,76 & 0,464 \\
\hline & KMF86 & 49,423 & 3,778 & 0,415 & 39,00 & 0,458 \\
\hline & KVOL86 & 71,692 & 4,917 & 0,564 & 39,75 & 0,437 \\
\hline & KL95 & 24,580 & 18,391 & 2,458 & 42,25 & 0,364 \\
\hline & $K L 86$ & 39,979 & 24,906 & 3,675 & 43,71 & 0,319 \\
\hline \multicolumn{7}{|c|}{ Fichte $\mathrm{N}=175$ (Fläche 130-133) } \\
\hline \multirow[t]{10}{*}{ GZ9499 } & KMF97 & 12,254 & 1,624 & 0,144 & 40,95 & 0,423 \\
\hline & KB97 & $-67,867$ & 36,032 & 3,226 & 41,10 & 0,419 \\
\hline & KSF97 & 5,973 & 5,315 & 0,479 & 41,23 & 0,415 \\
\hline & KVOL97 & 35,391 & 1,512 & 0,137 & 41,35 & 0,412 \\
\hline & KVOL92 & 29,539 & 1,250 & 0,128 & 43,29 & 0,356 \\
\hline & KMF92 & 11,947 & 1,236 & 0,131 & 43,82 & 0,340 \\
\hline & KB92 & $-62,515$ & 33,478 & 3,769 & 44,68 & 0,313 \\
\hline & KSF92 & 8,011 & 4,859 & 0,547 & 44,69 & 0,313 \\
\hline & KL97 & $-5,259$ & 15,006 & 1,695 & 44,73 & 0,312 \\
\hline & KL92 & 11,385 & 9,193 & 1,335 & 47,76 & 0,215 \\
\hline
\end{tabular}

$y=$ Zielvariable, $x=$ Regressor, $\beta_{0}=$ Achsenabschnitt, $\beta_{1}=$ Steigung, $s_{\beta_{1}}=$ Standardabweichung der Steigung, $M S_{\text {Res }}=$ Standardabweichung der Residuen, $R^{2}=$ Bestimmtheitsmaß

In der Tabelle 4.22 werden die Ergebnisse der vorher erläuterten einfachen Regressionsmodelle zur Schätzung des Grundflächenzuwaches mit multiplen und einfachen Regressionsmodellen unter Berücksichtigung des Belaubungsgrades verglichen. 
Tab. 4.22: Vergleich der linearen Regressionsmodelle zur Schätzung des Grundflächenzuwachses $(G Z)$ aus Kronenvariablen $(K S F, K M F, K V O L)$ und Belaubungs$\operatorname{grad}(B G)$

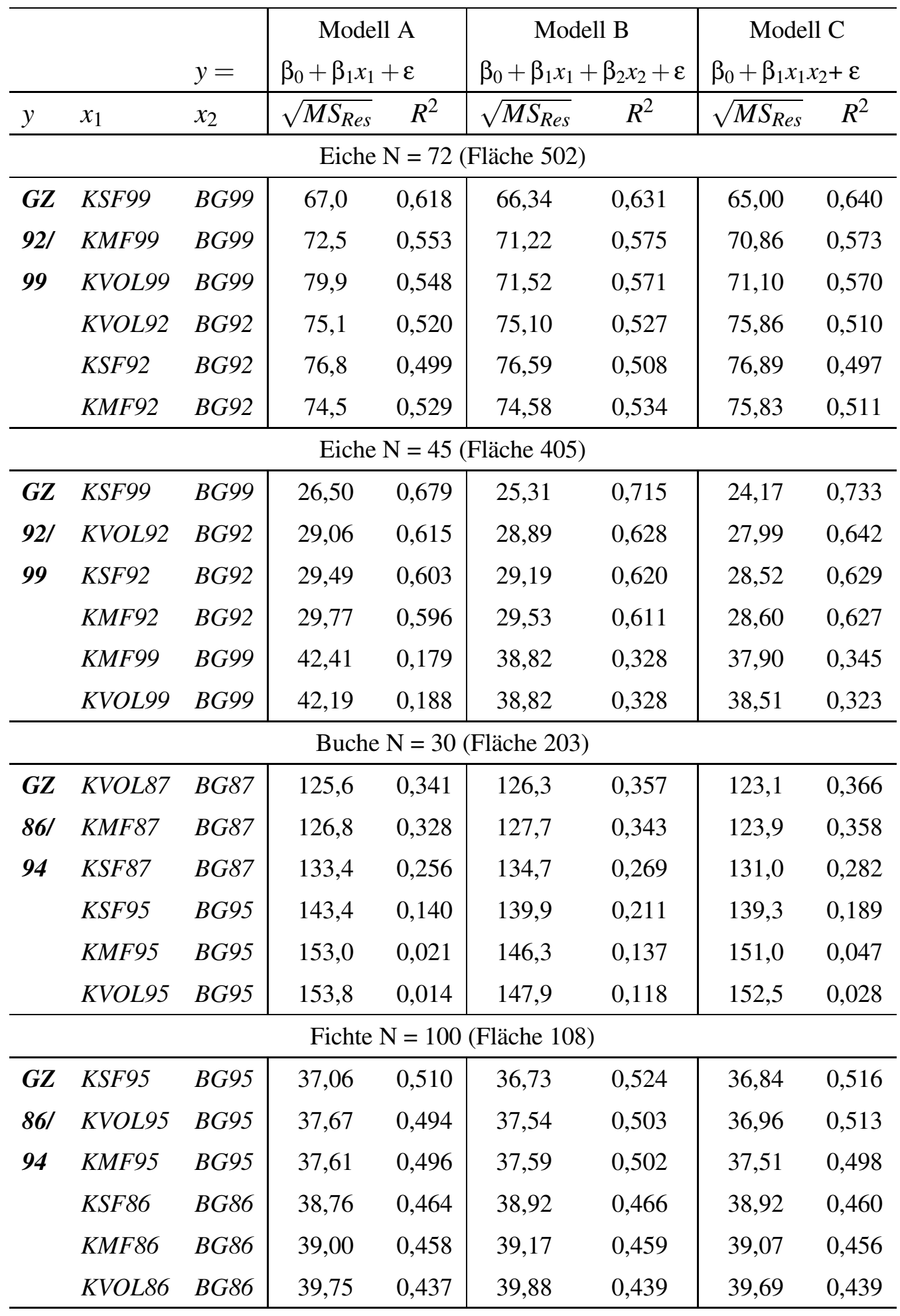

$y=$ Zielvariable, $x_{i}=$ Regressorvariable,$\sqrt{M S_{\text {Res }}}=$ Standardabweichung der Residuen, $R^{2}=$ Bestimmtheitsmaß 
Im Fall der Eichen der Flächen 405 verbessern Modell B und C sämtliche Schätzungen des Grundflächenzuwachses gegenüber Modell A. Bestimmtheitsmaße sind am höchsten und Standardabweichungen der Residuen sind am geringsten beim Modell C mit dem Produkt aus Kronendimensionen und Belaubungsgrad. Die aus dem Luftbild geschätzten Nadel- / Blattverluste verbessern hier eindeutig die Modelle. Bei den Eichen der Fläche 502 haben alle multiplen Modelle B höhere Bestimmtheitsmaße als Modell A. Modell C ist nur mit dem Produkt aus Kronenschirmfläche und Belaubungsgrad 1999 das beste Modell. Da gleichzeitig die Residualstreuung des multiplen Modelles B kleiner ist, als bei Modell A, ist auch hier eineVerbesserung der Schätzungen festzustellen, die jedoch sehr gering ausfällt.

Der Grundflächenzuwachs der Buchen auf der Fläche 203 zum Anfang der Untersuchungsperiode läßt sich am besten mit dem Modell C erklären. Zum Ende des Untersuchungszeitraumes im Jahr 1995 ist das beste Schätzmodell B; die Zusammenhänge sind aber insgesamt bei den Buchen sehr schwach.

Bei den Fichten auf Fläche 108 führt nur das Modell C mit den Kronenvolumen und Belaubungsgraden 1986 und 1995 und das Modell B mit den Kronenmantelflächen 1986 $\mathrm{zu}$ Verbesserungen.

Zusammenfassend läßt sich feststellen, dass von den insgesamt 24 Modellkombinationen 12 mal das Modell $\mathrm{C}$ höhere Bestimmtheitsmaße aufweist als das Modell A. In 11 Fällen ist das Bestimmtheitsmaß des Modelles B höher als das des Modelles A.

Da das Bestimmtheitsmaß mit der Anzahl der Regressionskoeffizienten ansteigt, ist eine Betrachtung der Residualstreuungen aufschlußreicher. Modell C weist bei 16 Modellkombinationen die geringsten Residualstreuungen auf. In drei Fällen wird die Residualstreuung durch Modell B reduziert.

In einigen Fällen kann der Nadel-/Blattverlust, der am Anfang oder Ende des Untersuchungszeitraumes beobachtet wird, zu einer Verbesserung der Schätzung des Grundflächenzuwachses beitragen, die aber insgesamt sehr gering ausfällt. 


\section{Diskussion}

Im Rahmen regionaler und internationaler Inventurprogramme besteht ein zunehmender Informationsbedarf in der Erfassung der räumlichen Verteilung und zeitlichen Entwicklung von Einzelbäumen. Die Informationserhebung auf permanenten Versuchsflächen zur langfristigen Umweltkontrolle oder für Zwecke der Waldwachstumsforschung konzentriert sich überwiegend auf terrestrische Erhebungen. Die Informationen aus Fernerkundungsdaten werden nur mit einer relativ geringen Intensität genutzt. Dieses trifft auch für Betriebsinventuren zu, in denen die Effektivität durch Luftbilderhebungen in einem zweiphasigen Stichprobenverfahren gesteigert wird (z. B. Böckmann et al. 1998).

Visuelle Bildinterpretationen und -messungen sind bisher übliche und operationell durchführbare Verfahren für die Erfassung von Einzelbäumen. Nachteile dieser Verfahren sind die Subjektivität der Interpreten, die langen Einarbeitungszeiten, die Begrenzung auf kleine Flächen und sich mehrfach wiederholende Routinearbeiten. Eine Erhöhung des computergestützten Anteiles der Luftbildauswertung, bis hin zu einer automatischen Auswertung, ist deshalb von praktischem Interesse. Orthophotos und Oberflächenmodelle als Produkte der digitalen Fotogrammetrie bieten hierzu neue Möglichkeiten.

Das in dieser Arbeit vorgestellte Konzept zur Verbesserung der digitalen Luftbildauswertung wurde in einer Fallstudie unter Verwendung eines Farbinfrarot-Stereomodelles im Maßstab 1:3100 an 285 Laubbäumen durchgeführt. Infolge des großen Maßstabes der Luftbilder wird nur eine geringe, stereoskopisch auswertbare Fläche abgebildet, die den praktischen Einsatzbereich auf Stichprobenverfahren oder die nähere Umgebung von Waldversuchsflächen begrenzt. Um Einflüsse der Sonnen- und Beobachtungsrichtung zu verringern, beschränken sich die digitalen Auswertungen auf einen Ausschnitt in der Nähe des Bildnadirs. Die Gültigkeit der nachfolgenden Vergleiche zwischen visuellen und digitalen Auswertungsverfahren ist durch weitere empirische Untersuchungen zu überprüfen.

Die Höhenfehler der digitalen Bildzuordnung, die exemplarisch an einigen Stereomodellen großmaßstäbiger CIR-Luftbilder untersucht wurden, sind im Wald größer als bei anderen Landbedeckungsklassen. Die theoretisch möglichen Höhengenauigkeiten einer visuellen fotogrammetrischen Punktmessung werden nicht erreicht. Sie liegen bei den untersuchten Stereomodellen in einer Größenordnung von 1 bis 2,5 m oder 1,0 2,5\%o der Flughöhe. Diese Ergebnisse bestätigen die Definitionsunsicherheit, die von Kraus (1997) für Bäume und Sträucher angegeben wird.

In beleuchteten Kronenteilen ist der Fehler der digitalen Höhenmodelle etwa zweimal größer als im Fall visueller fotogrammetrischer Messungen. Die Grenzen der Einsatzmöglichkeiten der verwendeten Bildzuordnungsalgorithmen sind besonders deut- 
lich in kontrastarmen Schattenbereichen und an inneren und äußeren Bestandesrändern, in denen es zu Fehlern in der Bildzuordnung kommt. Die Einstellgenauigkeit einer visuellen Stereoauswertung am analytischen Stereoplotter ist hier der automatischen Bildzuordnung weit überlegen. Dem steht eine hohe Punktdichte der automatischen Oberflächenmodelle gegenüber, die in visuellen Auswertungsverfahren nicht zu erreichen ist. Eine Möglichkeit der Steigerung der Genauigkeit der automatischen Höhenmodelle besteht in der Auswahl einer möglichst großen Bildbasis. Die relativ hohen Differenzen zwischen digitalen Höhenmodellen benachbarter Stereomodelle sind wahrscheinlich auf die geringe Basislänge bei einer Längsüberdeckung von $80 \%$ zurückzuführen. Mit einer Abstimmung der Scannerauflösung, der Auswahl geeigneter Bildkanäle und dem Ausschluß problematischer Schatten- und Randbereiche ist eine weitere Reduzierung der Fehler möglich.

Im Zuge der automatischen Luftbildinterpretation werden Merkmale definiert, die komplexe Objekte beschreiben. Darüber hinaus müssen Entscheidungsregeln gefunden werden, die zur Erkennung von Mustern oder zur Trennung verschiedener Objektklassen führen. Ein hierarchisches Vorgehen vom Einfachen zum Komplizierten hat sich hierbei bewährt.

In der ersten Stufe wurde deshalb der Überschirmungsgrad durch Trennung der Klassen „Krone“ und „Nicht-Krone“ bestimmt (vgl. Gougeon (2000), Gerylo et al. (1998)). Die Anwendung eines überwachten Klassifikationsverfahrens, unter Verwendung der spektralen Information der RGB-Kanäle des Orthofotos als auch der räumlichen Information eines normalisierten Oberflächenmodelles mit visueller Abgrenzung der Trainingsgebiete, ergab am Beispiel eines geschlossenen Laubholzbestandes reproduzierbare Ergebnisse in verschiedenen Bildjahrgängen. Konventionelle Klassifikationsverfahren weisen den Nachteil auf, dass sie eine Klassenzuordnung vornehmen, die nur auf den spektralen Werten des jeweiligen Pixels beruht. Die Berücksichtigung der Werte der Nachbarpixel beim SMAP-Algorithmus verbessert die Klassifikationsergebnisse gegenüber denen eines Maximum-Likelihood-Klassifikators.

Die systematischen Abweichungen der Ergebnisse einer digitalen Klassifikation des Überschirmungsgrades von $10-20 \%$ im Vergleich zu einer visuellen Interpretation sind auf Kronenteile im Schatten und in der Unterschicht des zweistufigen EichenBuchen-Bestandes zurückzuführen, die vom Auswerter visuell nicht eindeutig einer Krone in der Oberschicht zugeordnet werden konnten. Die Größe der Schattenbereiche wird u. a. auch vom Sonnenzenitwinkel beeinflußt. Wird der Überschirmungsgrad verschiedener Bildjahrgänge verglichen, so ist auf den Sonnenstand zum Zeitpunkt der Bildaufnahme zu achten.

Die ausschließliche Verwendung der Information eines normalisierten Oberflächenmodelles zur Klassifikation von Bestandeslücken, welches Hoffmann (2001) vorschlägt, ist von der Wahl eines Schwellenwertes abhängig, der die Berechnung einer mittleren Bestandeshöhe in homogenen, einschichtigen Waldbeständen voraussetzt. Für stärker vertikal strukturierte Waldbestände mit geringer Kronenüberschirmung wird vom $\mathrm{Au}-$ tor eine Kombination der RGB-Kanäle mit einem normalisierten Oberflächenmodell in einer überwachten Klassifikation vorgeschlagen. Verbesserte Ergebnisse für die Klassifikation der Vegetation in Stadtgebieten werden in dieser Kombination von Beisch 
(1998) bestätigt.

Eine Schlüsselfunktion der digitalen Auswertung von Luftbildern nimmt die nächste Stufe der Abgrenzung einzelner Baumkronen ein. Die Verwendung multispektraler Klassifikationstechniken führt nur zur Isolierung einzelner Kronen, wenn diese vollständig von Schattenbereichen umgeben sind. Bei den Laubholzkronen des Untersuchungsbestandes war dieses nicht der Fall. Als Ergebnis der Klassifikation des Überschirmungsgrades wurde eine Maske der Klasse Krone erstellt, welche Grundlage für eine automatische Segmentierung bildete.

Die grundsätzliche Eignung des Wasserscheidenverfahrens unter Verwendung eines digitalen Höhenmodelles kann in einem experimentellen Ergebnis für die Abgrenzung von Laubholzkronen bestätigt werden. Der verwendete Algorithmus besteht aus der Kombination der Ansätze des Gebietswachstums und der Kantenextraktion. Es entstehen geschlossene Polygone, die vorteilhaft für die Baumkronenerkennung genutzt werden können. Das Ergebnis der Segmentierung ist aber in hohem Maße von der räumlichen Struktur des digitalen Oberflächenmodelles abhängig. Die räumliche Struktur eines Höhenmodelles steht in Beziehung zur Größe der interessierenden Objekte (hier Baumkronen) und der räumlichen Auflösung (Kantenlänge der Pixel in Bodenauflösung) des verwendeten Bildmaterials. Die Skalenraum-Filterung (scale space filtering) baut auf theoretischen Erkenntnissen der fraktalen Geometrie auf. Als Glättungsfunktion der digitalen Höhenmodelle kann eine Folge von Gauß-Funktionen eingesetzt werden und damit störendes „Rauschen“ beseitigt werden. Die Bestimmung der Grundfläche des Filterkerns und des Skalierungsparameters lässt sich visuell durchführen. Für eine empirische oder automatische Herleitung der Skalenparameter wird auf Ansätze von Haberäcker (2000) und Lindeberg (1994) verwiesen.

Bei der Wahl eines zu kleinen Maßstabes bzw. einer zu kleinen Standardabweichung des Glättungsfilters entsteht eine Übersegmentierung mit zu kleinen Regionen innerhalb der Einzelkronen. Bei Auswahl einer zu großen Standardabweichung, werden nicht mehr einzelne Bäume abgegrenzt. Vielmehr kommt es zur Ausweisung ganzer Gruppen von Baumkronen. Scharfe Kanten bleiben auch bei größeren Standardabweichungen erhalten. Das Ergebnis des Wasserscheidenverfahrens ist nicht so zuverlässig wie eine konventionelle visuelle Stereokartierung. Die Algorithmen sollten durch die Formulierung zusätzlicher Bedingungen, die z. B. multispektrale Merkmale berücksichtigen, weiter verbessert werden (vgl. Gonzalez und Woods 2002). Für die nachfolgende objektbasierte Klassifikation und Bestimmung von Farb- und Formmerkmalen wurden deshalb visuell abgegrenzte Bildsegmente definiert.

Die Güte der Bildsegmentierung ist von großer Bedeutung für die Definition von baumkronenspezifischen Merkmalen und die anschließende objektbasierte Klassifikation. In dieser Arbeit wurden Farb-, Textur- und Formmerkmale definiert, die sich für eine Beschreibung und multivariate Klassifikation von Baumkronen eignen. Die wertvolle Information der Stereoskopie wird als digitales Oberflächenmodell genutzt.

Baumarten haben charakteristische Kronenformen und Verzweigungstypen, welche in den großmaßstäbigen Luftbildern zu erkennen sind. Dies spiegelt sich in zahlreichen Arbeiten wider, die sich mit der Interpretation von Baumarten in Luftbildern befassen (z. B. Rabenau und Blasbichler 1969, Sayn-Wittgenstein 1960, Baumann 1957). Die 
Interpretationsschlüssel beschreiben geometrische Formen und artspezifische Ausformungen der Kronenränder. Strukturen innerhalb der Baumkronen sind häufig erst in einem räumlichen Stereomodell deutlich zu erkennen.

Die Rauhigkeit von Baumkronen kann mit Hilfe digitaler Oberflächenmodelle charakterisiert werden. Ein Merkmal zur Beschreibung der gesamten Krone ist das Verhältnis der Kronenmantel- zur Kronenschirmfläche. Die Gaußsche Krümmung ist im weiteren Sinne ein Maß für die Abweichung einer gekrümmten Oberfläche von einer Ebene (im gegebenen Fall der Flächengröße $5 \times 5$ Pixel (entspricht 1,6 $\mathrm{m}^{2}$ der Bodenauflösung)). Hohe mittlere Absolutwerte der Gaußschen Krümmung einer Krone kennzeichen konvexe oder konkave Oberflächen. Die Modellierung der Kronenschirmfläche als Ellipse führt zur räumlichen Analyse der Kronenraumbesetzung verschiedener Baumarten. Hauptorientierung und Länglichkeit sind dabei interessante Möglichkeiten zur Beschreibung der Laubholzkronen.

Die Unterscheidung der Baumarten Buche und Eiche wurde mit einer klassischen linearen Diskriminanzanalyse durchgeführt. Die Anwendung weiterer multivariater Klassifikationsverfahren (z. B. allgemeine lineare Regressionsmodelle, neuronale Netze) ist ebenfalls möglich, da die aggregierten Segmentmerkmale in Listen vorliegen und in Statistik-Programmen weiterverarbeitet werden können. Kriterien zur Auswahl geeigneter segmentbeschreibender Attribute sind zu definieren. Die Merkmale Gaußsche Krümmung und Kronenrauhigkeit waren in diesem Zusammenhang besser geeignete Variablen als Farb- oder Texturmerkmale. Die Baumarten Eiche und Buche konnten mit einer Gesamtgenauigkeit von $89 \%$ getrennt werden.

Nach der Baumartenklassifikation wird in der letzten Stufe eine vierstufige Klassifikation der Vitalität nach der Kronenverlichtung vorgeschlagen. Die Stufe 4 mit totalem Nadel-/Blattverlust kann aufgrund der typischen spektralen Signaturen und mit Hilfe eines Schwellenwertverfahrenss identifiziert werden.

Eine Trennung von zwei Blattverluststufen der Baumart Eiche wurde mit einer Gesamtgenauigkeit von $79 \%$ durchgeführt. Die Ergebnisse bestätigen die Bedeutung der spektralen Information für die Extraktion von Kronenverlichtungsstufen. Methoden der digitalen Bildverbesserung können vorteilhaft für die Auswertung der digitalen Orthofotos eingesetzt werden. Die zweite Hauptkomponente der ursprünglichen Kanäle des Farbinfrarot-Orthofotos wurde als geeignete Variable für die Trennung der Kronenverlichtungsstufen von Eichen bestimmt. Scheer (2000) führte Signaturanalysen an Fichten mit verschiedenen Kronenverlichtungsgraden durch. Als Ergebnis einer Diskriminanzanalyse berichtet er ebenfalls über die Eignung der zweiten Hauptkomponente der Farbauszüge eines Orthofotos für Zwecke der Vitalitätsbeurteilung.

Für die Bestimmung der Textur wurden klassische Texturmerkmale nach Haralick et al. (1973) eingesetzt. Geeignete Variablen waren die Merkmale contrast und varian$c e$, die sich aus einer $3 \times 3$ Grauwert-Übergangsmatrix des nahinfraroten Kanales ableiten lassen. Die Güte der Klassifikation von Blattverluststufen der Eichen wird damit verbessert. Koch et al. (2002) untersuchten Texturparameter für die Unterscheidung von Nadel- und Laubholzarten und auf Basis von visuell delinierten Baumkronen in großmaßstäbigen CIR-Luftbildern. Sie empfehlen das Texturmerkmal contrast des grünen Spektralbereiches und mean (Mittelwert der Grauwert-Übergangsmatrix) des infraro- 
ten Spektralbereiches für die Baumartentrennung. Yuan et al. (1991) stellen straffe Zusammenhänge zwischen dem Schädigungsgrad von Ahorn-Bäumen als Ergebnis einer Luftbildinterpretation und dem Texturmerkmal contrast des grünen Spektralbereiches einer Videoaufnahme fest.

Die luftbildsichtbare Kronenmantel- und Kronenschirmfläche haben sich bei Eichen und Fichten als geeignete Variablen für die Schätzung des Grundflächenzuwachses erwiesen. Die Ergebnisse bestätigen die Untersuchungsergebnisse von Akça (1984) zur Eignung der fotogrammetrischen Kronengrößen zur Zuwachsschätzung bei Fichte. Die Kronendimensionen der Lichtbaumart Eiche sind mit Ausnahme der luftbildsichtbaren Kronenlänge ebenfalls geeignete erklärende Variablen. Ebert und Rieger (2000) stellen vergleichbare lineare Zusammenhänge zwischen terrestrisch erhobenen Kronengrößen und dem Grundflächenzuwachs von Eichen her und bevorzugen die Kronenmantelfläche als beste Variable zur Zuwachsschätzung. Die luftbildsichtbare Kronenlänge ist nach den Ergebnissen dieser Studie weniger gut zur Verbesserung der Schätzmodelle geeignet. Die fotogrammetrisch erfassten Kronenlängen der Fichten unterliegen in besonderem Maße den Beleuchtungseinflüssen. Ein geringer Sonnenzenitwinkel und die daraus resultierenden Schatten führen zu einer Unterschätzung der Kronendimensionen.

Die visuell im Luftbild geschätzten Nadel-/Blattverluste wurden als Belaubungsgrad zur Gewichtung der Kronendimensionen in verschiedenen Regressionsmodellen zur Schätzung des Grundflächenzuwachses eingeführt. Auf der Eichenfläche 405 konnten die Luftbildansprachen die Beziehungen zum Grundflächenzuwachs im Vergleich zu den univariaten Schätzmodellen mit einer Kronendimension verbessern. Bei den anderen untersuchten Flächen kann der Belaubungsgrad nur zu sehr geringen Verbesserungen der Schätzmodelle beitragen.

In der vorliegenden Arbeit wurden einige Methoden der Bildanalyse zur Erfassung von Bäumen vorgestellt. Es sind erste Schritte, die weitere empirische Untersuchungen und Entwicklung praktischer Algorithmen nach sich ziehen müssen. Die Segmentierung digitaler Bilder in einheitliche Bereiche und Beschreibung dieser Segmente sollten Schwerpunkte der Weiterentwicklung halbautomatischer Auswertungsverfahren sein. Objektorientierte multivariate Klassifikationsverfahren sind für die Informationsgewinnung für forstliche Zwecke von großer Bedeutung.

Die Methodenentwicklung der digitalen Bildanalyse zur Mustererkennung ist für medizinische, physikalische und industrielle Zwecke, z. B. für die Steuerung von Robotern oder zur industriellen Qualitätskontrolle, weit fortgeschritten. Die Übertragung auf Probleme der Fernerkundung und spezielle Anforderungen zur Erkennung von Einzelbäumen ist dagegen schwieriger, da vielfältige Aspekte der natürlichen Umwelt zu berücksichtigen sind.

Analoge Luftbildkameras werden heute weitgehend durch flugzeug- oder satellitengetragene digitale Flächensensoren ersetzt. Die Möglichkeiten der radiometrischen Kalibrierung dieser Sensoren lässt auf verbesserte Anwendungsmöglichkeiten der digitalen Bildverarbeitung für Zeitreihenauswertungen schließen. Mit aktiven Sensoren wie Laserscannern (LIDAR) können Waldoberflächenmodelle in Schatten- und Randbereichen besser erfasst werden. Aus der integrierten Auswertung räumlicher und multispek- 
traler Fernerkundungsaufzeichnungen werden neue Wege der Informationsgewinnung für Waldinventuren entstehen.

Die Untersuchungen wurden mit Programmen durchgeführt, die der freien SoftwareLizenz unterliegen und deren Quelltext für jedermann zugänglich ist. Die Technologie der Geoinformation setzt komplexe Programmsysteme der Datenbankverwaltung, Bildverarbeitung, Visualisierung und statistischen Analyse voraus, die nicht von Einzelpersonen entwickelt werden können. Die Software-Entwicklung wird über das Internet koordiniert und von vielen Nutzern und Entwicklern gemeinschaftlich weitergeführt. Die Erforschung neuer Algorithmen und Anwendungen wird in dieser offenen Umgebung gefördert, da Entwicklungszeiten durch Verwendung und Anpassung vorhandener Programmmodule erheblich verkürzt werden. Das Erlernen der Programmierung wird erleichtert und neue Impulse für eine optimierte Software-Entwicklung gegeben. 


\section{Zusammenfassung}

Seit 1994 wird das europäische Umweltmonitoring durch ein Programm zur intensiven Dauerbeobachtung von Waldökosystemen ergänzt (Level 2). Das Hauptziel des Level 2-Programmes ist die Beschreibung der Veränderungen von Ökosystemen, um zu einem besseren Verständnis natürlicher und anthropogener Faktoren beizutragen, welche die Wälder beeinflussen. Dazu dienen Erhebungen des Kronenzustandes, der Wachstums- und Ernährungssituation der Bäume und des Bodenzustandes sowie der Stoffeinträge und -austräge auf permanenten Beobachtungsflächen. Der Einsatz von Erhebungsmethoden der Fernerkundung ist optional. Ergänzend zu den terrestrischen Erhebungsmethoden wird der Zustand von Level 2-Flächen in Deutschland mit Hilfe großmaßstäbiger Farbinfrarot-Luftbilder dokumentiert.

Ziel der vorliegenden Untersuchung ist die Definition von Baummerkmalen aus digitalen Farbinfrarot-Luftbildern (Maßstab 1:2700 - 1:4300) und die Ableitung von Einzelbaum- und Bestandesvariablen, die relevant sind im Rahmen der Waldzustandserfassung des Level 2-Programmes. Zwei Flächen in Eichen-Buchen-Mischbeständen, zwei Flächen in Fichten-Reinbeständen und eine Fläche in einem Buchen-Reinbestand in Rheinland-Pfalz und Nordrhein-Westfalen wurden unter Verwendung von Methoden der Fotogrammetrie und Fotointerpretation untersucht.

Die Integration der geometrischen Information des digitalen Oberflächenmodelles und des multispektralen digitalen Orthofotos ist die Grundidee für ein neues Verfahren zur Gewinnung von baumspezifischen Informationen. Eine überwachte Klassifikation wird zur Unterscheidung zwischen Kronen und Nicht-Kronen-Bereichen eingesetzt. Eine visuelle Delinierung des Kronenrandes bildet die Grundlage für die Definition von baumkronenspezifischen Farb-, Textur- und Formmerkmalen. Der Einsatz des Wasserscheidenverfahrens unter Verwendung eines digitalen Oberflächenmodelles wird als vielversprechender Ansatz zur automatischen Segmentierung von Laubbaumkronen vorgestellt. Hierbei werden Kronenoberflächen als "Landschaft" aufgefasst, in welcher "Täler" und "Wasserscheiden" identifiziert werden, um einzelne Kronen zu beschreiben.

Die Ergebnisse einer Signaturanalyse identifiziert charakteristische Unterscheidungsmerkmale für die Baumarten Eiche und Buche. Formmerkmale wie die Gaußsche Oberflächenkrümmung oder Kronenrauhigkeit können die Baumartenunterscheidung gegenüber dem Einsatz von spektralen Merkmalen erheblich verbessern. Eine lineare Diskriminanzanalyse führte zu einer Trennung der Eichen und Buchen mit einer Gesamtgenauigkeit von $89 \%$.

Eine Klassifikation der Vitalität nach der Kronenverlichtung in vier Stufen wird vorgeschlagen. Das stehende Totholz (Stufe 4) wird aufgrund seiner charakteristischen spektralen Eigenschaften in einem einfachen digitalen Schwellenwertverfahren unter 
Verwendung eines Differenzindex (Rot minus Grün) und des NDVI (normaliserter DifferenzVegetationsindex) identifiziert. Eine lineare Diskriminanzanalyse ergab für die Trennung von 2 Kronenverlichtungsstufen der Eichen eine Gesamtgenauigkeit von $79 \%$, wobei sich der Einsatz der zweiten Hauptkomponente der RGB-Farbauszüge des Orthofotos als vorteilhaft erwies.

Für die Erfassung von Veränderungen ist die Genauigkeit von digitalen Oberflächenmodellen aus automatischer Bildzuordnung von Bedeutung. Es werden deshalb Methoden zur empirischen Überprüfung der vertikalen Genauigkeit angewendet mit dem Vergleich zwischen terrestrisch ermittelten Baumhöhen und Baumhöhen aus dem Luftbild, dem Vergleich zwischen digitalen Höhenmodellen dreier digitaler fotogrammetrischer Systeme mit visuellen Höhenmessungen an einem analytischen Stereoplotter und der Überprüfung der relativen Differenzen im mehrfachen Überdeckungsbereich benachbarter Stereomodelle. Automatisch generierte digitale Oberflächenmodelle weisen höhere Fehler auf als visuelle Stereomessungen. Die relativen Differenzen digitaler Oberflächenmodelle im Überdeckungsbereich benachbarter Modelle fallen unerwartet hoch aus, was wahrscheinlich auf den geringen Abstand der Projektionszentren der Stereobilder (geringe Basislänge, Längsüberdeckung ca. 80\%) zurückzuführen ist. Die Ergebnisse belegen eine systematische Unterschätzung der Luftbildhöhen im Vergleich zu terrestrischen Baumhöhen. Die relativen Differenzen sind in Waldbereichen höher als bei anderen Landbedeckungsklassen aufgrund von Schattenbereichen und Randeffekten. Die Beziehungen zwischen terrestrisch erhobenem Grundflächenzuwachs und Stammdurchmesser und im Luftbild ermittelten Kronendimensionen wurden mit linearen Regressionsanalysen untersucht. Hohe Bestimmtheitsmaße der Modelle Grundflächenzuwachs gegen Kronengrößen wurden für Eichen und Fichten festgestellt. Kronenmantelund -schirmfläche am Ende der Untersuchungsperiode waren die am besten geeigneten Variablen zur Schätzung des Grundflächenzuwachses. Die Beziehungen erweisen sich bei Buchen als schwächer. Der aus dem Luftbild geschätzte Belaubungsgrad von Einzelbäumen verbessert die Schätzmodelle des Grundflächenzuwachses nur geringfügig. 


\section{Abstract}

Since 1994 the European Environmental Monitoring has been complemented by a program of intensive surveys of forest ecosystems (Level 2). Major goal of the Intensive Monitoring Program is the assessment of changes of the forest through natural and human factors. On permanent observation plots variables such as crown condition, growth, soil condition, foliar nutrient status and atmospheric deposition are recorded. The application of Remote Sensing is optional. In Germany, in addition to the terrestrial surveys, large-scale CIR-aerial photographs were taken of these observation plots.

The objective of the present study is the measurement and estimation of tree characteristics from digital CIR-aerial photographs (scale 1:2700 - 1:4300) which are relevant for the Level 2 program. Two plots in oak-beech stands, two plots in spruce stands and two plots in a beech stand in the states of Rheinland-Pfalz and Nordrhein-Westfalen were analysed by using methods of photogrammetry and photointerpretation.

The integration of geometric information of digital surface models and multispectral digital orthophotos is the basic idea of a new approach to derive information of individual trees. A supervised classification was employed to distinguish crowns and background. A visual delineation of individual tree crowns is used as the base for the definition of colour, texture and shape characteristics. A promising approach is the automated watershed segmentation of broadleaved tree crowns. Crown surface models are understood as a landscape in which valleys and watersheds are identified to describe individual tree crowns.

Distinct features are identified analysing the signatures of oaks and beechs. Shape pattern of tree crowns such as the Gaussian curvature or crown roughness are suitable to improve separation of tree species. Linear discriminant analysis resulted in the identification of oaks and beechs with an overall accuracy of $89 \%$. Four classes to estimate the defoliation of oaks are proposed by the author. Dead standing trees are easily identified by spectral information using thresholds based on a difference index (red minus green) and the NDVI (normalized difference vegetation index). The second principle component of the RGB components of the digital orthophoto is suitable for the separation of two defoliation classes of oaks by a linear discriminant analysis with an overall accuracy of $79 \%$.

For reasons of change assessment the quality of automatic digital surface models from image matching was evaluated. Analysis of the accuracy was performed by comparing terrestrial and aerial tree heights, comparing automatic elevation models of three digital photogrammetric workstation with manual measurements of an analytical stereoplotter and by checking the relative fit of heights in the overlap-region between neighbouring models. Relative differences of digital elevations in the overlap-area bet- 
ween neighbouring models were unexpected large probably due to the small distance between the position of the camera of the stereo images (short length of base, end lap c. $80 \%$ ). The results indicate a systematic underestimation of aerial broadleaved tree heights. The relative differences of elevations in forested areas were larger compared to other landcover classes, the causes being shadows and border effects.

The relations between basal area growth and aerial crown variables were investigated using linear regression analysis. High coefficients of determination were found for models of basal area growth versus crown dimensions for oak and spruce. Crown surface area and crown area were the explanatory variables of the best models. The relationships were not so strong with beech trees. The visual interpretation of defoliation contributed little to the estimation of the basal area growth. 


\section{Literaturverzeichnis}

Ackermann und Hartmann 1992 Ackermann, J. ; Hartmann, G.: Kronenschäden in Eichenbeständen Niedersachsens nach Farbinfrarot-Luftbildern aus den Jahren 1988/89. In: Forst und Holz 47 (1992), S. 452-460

Ackermann et al. 1996 ACKermann, J. ; Ohlhoff, R. ; Fuchs, H. ; Evers, J.: Großräumige Erfassung der Bestandesverlichtung und -auflösung in Luftbildern. In: Allgemeine Forstzeitschrift für Waldwirtschaft und Umweltvorsorge (1996), Nr. 2, S. $62,64-65$

Adler 2001 ADLER, P.: Einsatz digitaler Photogrammetrie zur Beschreibung von Waldbeständen : am Beispiel der digitalen photogrammetrischen Erfassung der Level 2 Flächen Baden-Württembergs, Univ. Freiburg, Forstwiss. Fak., Dissertation, 2001

ADW 1997 ADW ; ARBEITSGEMEINSCHAFT DAUERBEOBACHTUNGSFLÄCHENWALDSCHÄDEN DER LÄNDER UND DES BUNDES (Hrsg.): Waldbäume: Bildserien zur Einschätzung von Kronenverlichtungen bei Waldbäumen. Faste, 1997

AFL/VDI 1993 AFL/VDI ; ARBEITSKREIS FORSTLICHER LUFTBILDINTERPRETEN / Verein DeUtSCher INGENIEURE (Hrsg.): Messen von Vegetationsschäden am natürlichen Standort. Interpretationsschlüssel für die Auswertung von CIRLuftbildern zur Kronenzustandserfassung von Nadel- und Laubbgehölzen. Berlin : Beuth, 1993 (VDI-Richtlinie 3793)

Akça et al. 1995 AKÇA, A. ; FeldKötter, C. ; Henjes, M.: Auswertung einer CIR-Luftbild-Zeitreihe (1991-1994) des Fichten-Kalkungsversuchs an der Waldmeßstation Elberndorf im Hochsauerland. Inst. für Forsteinrichtung und Ertragskunde, Univ. Göttingen, 1995 (Abschlußbericht imAuftrag der LÖBF/LAfAO). - Unveröffentlicht

Akça et al. 2000 AKÇA, A. ; Fuchs, H. ; Cho, H.-K. ; KAhle, M. ; WeGNER, B.: Untersuchungen zum Zusammenhang von Kronenzustand und Zuwachs durch photogrammetrische Kronenvermessung auf Basis einer CIR-Luftbildreihe an Waldökoystem-Dauerbeobachtungsfächen im Forstamt Merzalben (Eiche 405) und Forstamt Hermeskeil (Fichte 130-133). Institut für Forsteinrichtung und Ertragskunde, Universität Göttingen, 2000 (Abschlußbericht im Auftrag der Forstlichen Versuchsanstalt Rheinland-Pfalz). - Unveröffentlicht 
Akça 1970 AкÇA, A.: Eine Untersuchung zur Unterscheidung und Identifizierung einiger Objekte aud Schwarz-Weiß-Luftbildern durch quantitative Beschreibung der photographischen Textur, Univ. Freiburg, Dissertation, 1970

Akça 1979 AKÇA, A.: Aerophotogrammetrische Messung der Baumkronen. In: Deutscher Verband Forstl. Forschungsanst., Mehring (1979), S. 79-91

Akça 1984 AKÇA, A.: Zur Zwachsschätzung mit Hilfe von Kronenmessungen im Luftbild. In: Allg. Forst.- u. J.-Ztg. 155 (1984), Nr. 6, S. 136-140

Akça 1989 AKÇA, A.: Permanente Luftbildstichprobe. In: Allgemeine Forst- und Jagdzeitung 160 (1989), Nr. 4, S. 65-69

Akça et al. 2002 AKÇA, A. ; Fuchs, H. ; Wegener, B.: Untersuchungen zum Zusammenhang von Kronenzustand und Zuwachs durch photogrammetrische Kronenvermessung auf Basis einer CIR-Luftbildreihe an der WaldökosystemDauerbeobachtungsfläche Eiche 502 Tannenbusch im Forstamt Kleve. Institut für Waldinventur und Waldwachstum, Univ. Göttingen, 2002. - Unveröffentlicht

Akça et al. 1971 AKÇA, A. ; Hildebrandt, G. ; Reichert, P.: Baumhöhenbestimmung aus Luftbildern durch einfache Parallaxenmessung. In: Forstwissenschaftliches Centralblatt (1971), S. 201-215

Akça et al. 1990 AKÇA, A. ; PAhl, A. ; Setje-Eilers, U.: Auswertung einer CIR-Luftbild-Zeitreihe des Fichten-Kalkungsversuchs im Forstamt Hilchenbach Nordrhein-Westfalen. 1990. - Untersuchung im Auftrag der LÖLF. Inst. für Forsteinrichtung und Ertragskunde der Univ. Göttingen. Unveröffentlicht

Akça et al. 1991 AKÇA, A. ; Rhein, M. ; Setje-Eilers, U.: Erfassung und Beobachtung eines Fichtenbestandes im Forstamt Hilchenbach NRW mittels Auswertung einer CIR-Luftbild-Zeitreihe und Regressionsanalyse der Beziehungen zwischen Grundfächenzuwachs, Kronenparametern und Schädigung. 1991. - Untersuchung im Auftrag der LÖLF. Inst. für Forsteinrichtung u. Ertragskunde d. Univ. Göttingen. Unveröffentlicht

Al-Rousan et al. 1997 Al-Rousan, N. ; Cheng, P. ; Petrie, G. ; Toutin, Th. ; VALADAN ZOEJ, M.J.: Automated DEM Extraction and Orthoimage Generation from SPOT Level 1B Imagery. In: Photogrammetric Engineering \& Remote Sensing 63 (1997), Nr. 8, S. 965-974

Albertz 1998 Albertz, J.: Methoden der Mustererkennung. In: PfeIL (Hrsg.): Rundgespräche der Kommission für Ökologie: Fernerkundung und ÖkosystemAnalyse Rundgespräch am 26. Und 27. Oktober 1998. München, 1998, S. 37-51

Alexandrov et al. 1989 Alexandrov, A.D. ; Kolmogorov, M.A. ; LAVRENT'EV, M.A.: Mathematics. Its content, methods, and meanings.. Bd. 2. Cambridge : MIT Press, 1989 
Anonymus 2002 ANONYMUS: Manual on methods and criteria for harmonized sampling, assessment, monitoring and analysis of the effects of air pollution on forests Part II: Visual Assment of Crown Condition and Submanual on Visual Assessment of Crown Condition on Intensive Monitoring Plots. United Nations Economic Comission for Europe Convention on Long-Range Transboundary Air Pollution Effects on Forests. 2002. - URL http://www.icp-forests.org/Manual.htm

Arge et al. 2001 Arge, L. ; Chase, J.S. ; Halpin, P. ; Toma, L. ; VitTer, J.S. ; Urban, D. ; WiCKremesighe, R.: Flow Computation on Massive Grid Terrains. In: ACM Symposium on Advances in Geographic Information Systems, URL http: / / www. bowdoin.edu/ltoma/papers/terraflow-geoinformatica.pdf, 2001

Austin 1984 Austin, R.F.: Spatial Statistics and Models. Kap. Measuring and comparing two-dimensional shapes, S. 293-312, Reidel, 1984

Autometric Inc. 1998 Autometric InC.: Softplotter User Manual Version 1.8.3. Autometric Inc. 1998

Baatz und Schäpe 2000 BAATZ, M. ; SCHÄPE, A.: Angewandte Geographische Informationsverarbeitung XII: Beiträge zum AGIT-Symposium Salzburg 2000. Kap. Multiresolution Segmentation - an optimization approach for high quality multi-scale image segmentation, S. 12-23, Wichmann, 2000

Bahrenberg et al. 1992 Bahrenberg, G. ; Giese, E. ; Nipper, J.: Statistische Methoden in der Geographie. 1. Stuttgart : Teubner, 1992

Baltsavias und Käser 1998 BALTSAVIAS, E. ; KÄSER, C.: DTM and Orthoimage Generation - a Thorough Analysis and Comparison of four digital Photogrammetric Systems. In: International Archives of Photogrammetry and Remote Sensing 32 (1998), Nr. 4, S. 42-51

Bannari et al. 1995 BANNARI, A. ; MORIN, D. ; BONN, F.: A review of vegetation indices. In: Remote Sensing Reviews 13 (1995), S. 95-120

Baraldi und Parmiggiani 1995 BARALDI, A. ; PARMiggiani, F.: An Investigation of the textural characteristics associated with gray level cooccurrance matrix statistical parameters. In: IEEE Transactions on Geoscience and Remote Sensing 33 (1995), Nr. 2, S. 293-304

Baron und Li 2001 BARON, J. ; LI, Y.: Notes on the use of $R$ for psychology experiments and questionnaires. 2001. - URL http://lib.stat.cmu.edu/R/CRAN/doc/contrib/Baron-rpsych.pdf

Baumann 1957 Baumann, H.: Forstliche Luftbildinterpretation. Schriftenreihe der Landesforstverwaltung Baden-Württemberg. 1957 
Baumgarten 1990 BAUMGARTEN, R.: Untersuchungen zur Waldschadensklassifizierung der Buche an Hand multispektraler Scannerdaten. Oberpfaffenhofen : DLR Institut für Optoelektronik, 1990 (Forschungsbericht DLR-FB90-56)

Beisch 1996 BEISCH, Th.: Erfassung der Vitalität durch spektrale Analyse digitalisierter Farbinfrarot-Luftbilder. In: Beiträge zur Waldinventur, Festschrift zum 60, Geburtstag von Prof. Dr. Alparslan Akca. Institut für Forsteinrichtung und Ertragskunde, Universität Göttingen, 1996, S. 1-12

Beisch 1998 BEISCH, Th.: Städtische Baum- und Grünflächeninformationssysteme, Univ. Göttingen, Dissertation, 1998

BMELF 1994 BMELF: Dauerbeobachtungsflächen in Deutschland. Beitrag zum europäischen Waldschadensmonitoring (Level II-Programm). BMELF. 1994

BMELF 1997 BMELF: Dauerbeobachtungsfächen zur Umweltkontrolle im Wald. Level II. Erste Ergebnisse. Bundesministerium für Ernährung, Landwirtschaft und Forsten. 1997

Böckmann et al. 1998 Böckmann, Th. ; SABOrowski, J. ; NAGEL, J. ; SPelLMANN, H.: Die Weiterentwicklung der Betriebsinventur in Niedersachsen. In: Forst und Holz 53 (1998), Nr. 8, S. 219-226

Bortz 1999 BORTZ, J.: Statistik für Sozialwissenschaftler. 5. Berlin : Springer, 1999

Böttcher und Kleinn 2003 BÖTTCHER, K. ; KLEINN, Ch.: Using crown surface models for the extraction of forest inventory information: forst experiences with study areas in state of Mecklenburg-Vorpommern. In: HYYPPÄ, J. (Hrsg.) ; NAESSet, E. (Hrsg.) ; Olssson, H. (Hrsg.) ; GranQvist Pahlen, T. (Hrsg.) ; ReEse, H. (Hrsg.): Proceedings of the ScandLaser Scientific Workshop on Airborne Laser Scanning of Forests, September $3 \& 4$, Umea, Sweden, Swedish University of Agricultural Sciences, Department of Forest Resource Management and Geomatics, 2003 (Working Paper 112), S. 189-197

Bouman und Shapiro 1994 Bouman, C.A. ; ShAPIRO, M.: A multiscale random field model for Bayesian Image Segmentation. In: IEEE Transactions on Image Processing 3 (1994), Nr. 2, S. 162-177

Brandtberg 1999 BRAndtBerg, T.: Automatic Individual Tree-Based Analysis of High Spatial resolution Remotely Sensed Data, Swedish University of Agricultural Sciences Uppsala, Dissertation, 1999

Brang 1998 BRANG, P. ; UMWELT, Wald und L. Bundesamt für (Hrsg.) ; WALD, Schnee und L. Eidgenössische Forschungsanstalt für (Hrsg.): Berichte der Eidgenössischen Forschungsanstalt für Wald, Schnee und Landschaft. Bd. 345: SanasylvaBericht 1997. Zustand und Gefährdung des Schweizer Waldes-eine Zwischenbilanz nach 15 Jahren Waldschadenforschung. 1998 
Burger 1939 BURGER, H.: Baumkrone und Zuwachs in zwei hiebsreifen Fichtenbeständen. In: Mitteilung der Schweizerischen Anstalt für das Forstliche Versuchswesen, Zürich 21 (1939), S. 147-176

Burger 1940 BURGER, H.: Holz, Blattmenge und Zuwachs. Ein 80-jähriger Buchenbestand. In: Mitteilung der Schweizerischen Anstalt für das Forstliche Versuchswesen, Zürich 21 (1940), Nr. 2, S. 329

Burger 1947 Burger, H.: Holz, Blattmenge und Zuwachs. Die Eiche. In: Mitteilung der Schweizerischen Anstalt für das Forstliche Versuchswesen, Zürich 25 (1947), Nr. 1, S. 251

Buschmann und Nagel 1992 Buschmann, C. ; NAGEL, E.: Reflexionsspektren von Blättern und Nadeln als Basis für die physiologische Beurteilung von Baumschäden. Karlsruhe : KFK-PEF, 1992 (Forschungsbericht 90)

Ciesla 1974 CIESLA, W.M.: Forest Insect Damage from High Altitude CIR-Photos. In: Photogrammetric Engineering and Remote Sensing 40 (1974), S. 683-689

Clausi 2002 Clausi, D.: An analysis of co-occurrence texture statistics as a function of grey level quantization. In: Canadian Journal of Remote Sensing 28 (2002), Nr. 1, S. $45-62$

Cohen 1960 Cohen, J.: A coeffizient of agreement for nominal scales. In: Educational and Psychological Measurement 20 (1960), S. 37-46

Culvenor 2003 CUlvenor, D.S.: Extracting individual tree information. In: WULDER, M.A. (Hrsg.) ; FRANKLIN, E.F. (Hrsg.): Remote Sensing offorest environments: concepts and case studies. Dordrecht, Netherlands : Kluwer, 2003, S. 255-277

Dralle 1997 DRALLE, K.: Locating trees by digital image processing of aerial photos. Horsholm, Danish Forest and Landscape Research Institute, PhD Thesis, 1997

Dreschler 1981 DRESCHLER, L.: Ermittlung markanter Punkte auf den Bildern bewegter Objekte und Berechnung einer 3D-Beschreibung auf dieser Grundlage, Universität Hamburg, Dissertation, 1981

Duden 2000 Duden ; Wissenschaftlicher RAT DER DUdEnREDAKTION (Hrsg.): Die deutsche Rechtschreibung. 22. Mannheim : Dudenverlag, 2000 (CD$\mathrm{ROM})$

Eastman Kodak Company 2000 EASTMAN KODAK COMPANY: Kodak Aerochrome II Infrared Film 2443 Kodak Aerochrome II Infrared NP Film SO-134. 2000. URL http: //www. kodak. com. - Aerial Data AS-69

Ebert und Rieger 2000 EBERT, H.-P. ; RIEGER, T.: Die Baumkrone als Maßstab für den Zuwachs von Eiche. In: Allgemeine Forstzeitung / Der Wald 8 (2000), S. 403406 
Essery und Morse 1992 ESSERY, C.I. ; MORSE, A.P.: The impact of ozone and acid mist on the spectral reflectance of young Norway spruce trees. In: International Journal of Remote Sensing 13 (1992), S. 3045-3054

Eule 1959 EuLE, H.-W.: Verfahren zur Baumkronenmessung und Beziehungen zwischen Kronengröße, Stammstärke und Zuwachs bei der Rotbuche. In: Allgemeine Forst- und Jagdzeitung 130 (1959), Nr. 7

Feldkötter et al. 1996 FeldKÖTter, C. ; Henjes, M. ; LuCASCHEwsKi, I.: Analytische Auswertung einer Luftbildzeitreihe zum Nachweis der Veränderungen der Vitalitäts- und Wachstumsparameter eines Fichten-Kalkungsversuches. In: BEISCH, T. (Hrsg.) ; АKÇA, A. (Hrsg.) ; KoMP, K.U. (Hrsg.): Fernerkundung und GeoInformationssysteme in der Ökologie der Landoberfläche. Göttingen : Cuvillier, 1996

FGDC 1998 FGDC: Geospatial Positioning Accuracy Standards, Part 3: National Standard for Spatial Data Accuracy. Federal Geographic Data Commitee, U.S. Geological Survey,. 1998. - URL http://fgdc.er.usgs.gov/fgdc.html. - FGDCSTD-007.3-1998

Fischer und von Kienlin 1987 Fischer, J. ; KIENLIN, A. von: Korrektur von Störfaktoren bei der Wald-Schadenserfassung unter Verwendung digitaliserter Luftbilder. In: Bildmessung und Luftbildwesen 2 (1987), S. 50-59

Förstner 1982 FÖRSTNER, W.: On the geometric precision of digital correlation. In: International Archives of Photogrammetry and Remote Sensing 24 (1982), Nr. 3, S. $176-189$

Franklin 2001 FrankLIN, S.E.: Remote Sensing for Sustainable Forest Management. Boca Raton : Lewis, 2001

Fuchs und Cho 2001 FUCHS, H.-J. ; CHO, H.-K.: Waldinventur, Waldwachstum und Forstplanung - Moderne Technologien, Methoden und Verfahrensweisen - Festschrift zum 60. Geburtstag von Prof. Dr. Klaus von Gadow. Kap. Analyse von Waldstrukturen mit Hilfe von großmaßstäbigen Farbinfrarot-Luftbildern am Beispiel einer Level 2-Fläche (Eiche 502), S. 17-30, Zohab, 2001

Gausman 1977 Gausman, H.W.: Reflectance of leaf components. In: Remote Sensing of Environment 6 (1977), S. 1-9

GDT 2002 GDT ; GRASS DEVELOPMENT TEAM (Hrsg.): Grass 5.0 Users Manual. Trento, Italy : ITC-irst, 2002. - URL http://grass.itc.it/gdp/html_grass5/

Gerylo et al. 1998 Gerylo, G. ; Hall R.J. ; Franklin S.E. ; Roberts A. ; Milton E.J.: Hierarchical Image Classification and Extraction of Forest Species Composition and Crown Closure from Airborne Multispectral Images. In: Canadian Journal of Remote Sensing 24 (1998), Nr. 3 
GNU project 2002 GNU PROJECT: The Free Software Definition. 2002. - URL http://www.gnu.org

Gonzalez und Woods 2002 GonZALEZ, R.C. ; WoODS, R.E.: Digital image processing. Upper Saddle River, New Jersey : Addison-Wesley, 2002

Gooch und Chandler 2001 GoOCH, M.J. ; CHAndLER, J.H.: Failure prediction in automatically generated digital elevation models. In: Computers \& Geosciences 27 (2001), S. 913-920

Gougeon 2000 GougEON, F.A.: Towards semi-automatic forest inventories using individual tree crown (itc) recognition. In: Technology Transfer Note, Forestry Research Applications, Pacific Forestry Center, Cannada (2000), Nr. 22, S. 6. - 1-5

GRASS Development Team 2002 GRASS DEVELOPMENT TEAM: What is Grass? 2002. - URL http://grass.itc.it/general.html

Gülch 1994 GüLCH, E.: Erzeugung digitaler Geländemodelle durch automatische Bildzuordnung. München, Universität Stuttgart, Deutsche Geodätische Kommission bei der Bayerischen Akademie der Wissenschaften : Reihe C, Dissertationen ; 418, 1994

Haala und Brenner 1999 HaAla, N. ; BRenner, C.: Extraction of buildings and trees in urban environments. In: ISPRS Journal of Photogrammetry \& Remote Sensing 54 (1999), S. 130-137

Haberäcker 2000 HABERÄCKER, P.: Praxis der digitalen Bildverarbeitung und Mustererkennung. München : Fachhochschule München, Fachbereich Informatik/Mathematik, 2000

Haenel et al. 1988 Haenel, S. ; TrÄnkner, H. ; Eckstein, W.: Automatische Baumkronen-Entdeckung im Luftbild. In: DFVLR-TAGUNGSBÜRO WALDSCHÄDEN (Hrsg.): 2. DFVLR-Statusseminar: Untersuchung und Kartierung von Waldschäden mit Methoden der Fernerkundung, Oberpfaffenhofen, 30. November - 2. Dezember 1987. Köln, 1988

Halbritter 1996 HALBRITTER, K.: Steps towards a forest biodiversity information system: quantifying forest biodiversity by photogrammetry and remote sensing methodology. In: TECHNICAL UNIVERSITY IN ZvOLEN (Hrsg.) ; FACULTY OF FOREStRY (Hrsg.) ; DePartMent of Forest MANAGEMENT AND GEODESY (Hrsg.): Application of Remote Sensing in Forestry, II. International Symposium, Zvolen, September 10 - 12, 1996, 1996, S. 119-133

Haralick et al. 1973 Haralick, R.M. ; Shanmugam K. ; Dinstein, I.: Textural features for image classification. In: IEEE Transactions on Systems, Man, and Cybertinetics SMC-3 (1973), Nr. 6, S. 610-621 
Haralick und Shapiro 1992 Haralick, R.M. ; Shapiro, L.G.: Computer and robot vision. Bd. 1. Reading, Mass. : Addison-Wesley, 1992

Heipke 1995 HEIPKE, C.: Digitale photogrammetrische Arbeitsstationen. München : Deutsche Geodätische Kommission bei der Bayerischen Akademie der Wissenschaften, 1995 (C 450)

Heipke 1996 HEIPKE, C.: Overview of Image Matching Techniques. In: MEYLAN, D. (Hrsg.): OEEPE Workshop on the Application of Digital Photogrammetric Workstations March 4-6, 1996, Lausanne, Ecole Polytechnique Fédérale de Lausanne, 1996. - URL http://phot.epfl.ch/workshop/wks96/tocwks96.html

Heipke 2001 HEIPKE, C.: Digital Photogrammetric Workstations - A review of the state-of-the-art for topographic applications. In: GIM International 15 (2001), Nr. 4, S. 35-37

Henninger und Hildebrandt 1980 Henninger, J. ; Hildebrandt, G.: Bibliography of publications on damage assessment in forestry and agriculture by remote sensing techniques. 2. Freiburg : Universität Freiburg, 1980

Herrmann 1989 HERRMANn, K.: Signaturanalysen und Klassifizierungsergebnisse an Kiefern unter Verwendung multispektraler Flugzeug-Scannerdaten, Universität Münchnen, Dissertation, 1989

Herwitz et al. 2000 Herwitz, S.R. ; SANDlER, B. ; SLYE, R.E.: Twenty-one years of crown area change in the Jasper Ridge Biological Preserve based on georeferenced multitemporal aerial photographs. In: International Journal of Remote Sensing 21 (2000), Nr. 1, S. 45-60

Heurich et al. 2003 Heurich, M. ; Schneider, T. ; Kennel, E.: Laser scanning for identification of forest structure in the bavarian forest national park. In: HYPPÄ, J. (Hrsg.) ; NAesset, H. (Hrsg.) ; Olssen, H. (Hrsg.) ; GranQVist Pahlen T. (Hrsg.) ; REESE H. (Hrsg.): Proceedings of the ScandLaser Scientific Workshop on Airborne Laser Scanning of Forests, September 3 \& 4, 2003, Umea, Sweden, Swedish University of Agricultural Sciences, Department of Forest Resource Management and Geomatics, 2003, S. 98-107

Heydecke und Fuchs 2000 Heydecke, H. ; Fuchs, H.: Krone: Programm zur Ermittlung von Kronenparameter aus Luftbildmessungen. 2000. - Unveröffentlicht

Hildebrandt 1996 HILDEBRANDT, G.: Fernerkundung und Luftbildmessung: für Forstwirtschaft, Vegetationskartierung und Landschaftsökologie. Heidelberg : Wichmann, 1996

Hildebrandt und Kenneweg 1968 Hildebrandt, G. ; Kenneweg, H.: Einige Anwendungsmöglichkeiten der Falschfarbenphotographie im forstlichen Luftbildwesen. In: Allgemeine Forst- und Jagdzeitung 40 (1968), S. 205-213 
Hill und Leckie 1998 HILl, D.A. (Hrsg.) ; LECKIE, D.G. (Hrsg.) ; Canadian Forest Service (Veranst.): Proceedings of the International Forum on Automated Interpretation of High Spatial Resolution Digital Imagery for Forestry. Feb. 10-12. 1998. Victoria, B.C., Canada, 1998

Hoffmann 2001 Hoffmann, B.: Untersuchungen zum Monitoring von Naturwäldern mit Hilfe von Geo-Informationssystemen, modernen Luftbildauswertungsverfahren und Geostatistik, Universität Göttingen, Dissertation, 2001

Hoffmann und Kirchhof 1989 Hoffmann, H. ; KIRCHHOF, W.: Vergleichende reflexionsspektroskopische Vegetationsmessungen im Labor und Gelände an Rotbuchen- und Fichtenzweigen: Teil I. Versuchsaufbau, Labormessungen, Modellierung. Oberpfaffenhofen : Deutsche Forschungs- und Versuchsanstalt für Luftund Raumfahrt, 1989 (DLR-IB-552-8/89)

Holopainen und Wang 1998 Holopainen, M. ; WAng, G.: The calibration of digitzed aerial photographs for forest stratification. In: International Journal of Remote Sensing 19 (1998), Nr. 4, S. 677-696

Huberty 1994 HuberTy, C.J.: Applied Discriminant Analysis. New York : Wiley, 1994 (Wiley series in probability and mathematical statistics. Applied probability and statistics)

Hussein et al. 2000 Hussein, K.A. ; Albert, M. ; Gadow, K.v.: The crown window- a simple device for measuring tree crowns. In: Forstwissenschaftliches Centralblatt 119 (2000), S. 43-50

ICP-FOREST 1994 ICP-FOREST: Manual on methods and criteria for harmonized sampling, assessment, monitoring and analysis of the effects of air pollution on forests. UNECE, Hamburg. 1994. - URL http://www.icp-forests.org/Manual.htm

ICP-FOREST 2000 ICP-FOREST: Political Background. Bundesanstalt für Forst- und Holzwirtschaft, 2000. http://www.icp-forests.org/Political.htm

Jacobsen 2001 JACOBSEN, K.: PC-based Digital Photogrammetry. In: Workshop on Data Analysis and Image Processing Techniques Damascus, GORS 25-29 March 2001 UN/COSPAR/ESA/GORS (Veranst.), 2001

Jacobsen 2002 JACOBSEN, K.: Block Adjustment. Institute for Photogrammetry and Surveying Engineering, Univ. Hannover. 2002. - URL http://www.ipi.uni-hannover.de

Jensen 1996 JENSEN, J. R.: Introductory Digital Image Processing: a Remote Sensing Perspective. Prentice Hall, 1996 
Kahleys und Klier 1968 KAHLEYS, D. ; KLIER, G.: Untersuchungen zur Ermittlung holzmesskundlicher Daten aus Luftbildern bei der Baumart Fichte. In: Archiv für das Forstwesen 17 (1968), Nr. 4, S. 369-379

Kahmen 1993 Kahmen, H.: Vermessungskunde. 18. Berlin : de Gruyter, 1993

Kasser und Egels 2001 KASSER, M. ; EgELS, Y.: Digital Photogrammetry. London : Taylor \& Francis, 2001

Kätsch und Stöcker 2000 KÄTSCH, C. ; STÖCKER, M.: Untersuchungen zur automatischen Ermittlung von Bestandeshöhen auf Luftbildern mit Hilfe der Digitalen Photogrammetrie. In: Allg. Forst- u. J.-Ztg. 171 (2000), Nr. 4, S. 74-80

Kenneweg 1972 Kenneweg, H.: Die Verwendung von Farb- und InfrarotLuftbildern für Zwecke der forstlichen Photointerpretation unter besonderer Berücksichtung der Erkennung und Abgrenzung von Kronenschäden in Fichtenbeständen, Universität Freiburg, Dissertation, 1972

Kenneweg et al. 1991 Kenneweg, H. ; Foerster, B. ; Runkel, M.: Diagnose und Erfassung von Waldschäden auf der Basis von Spektralsignaturen. Teil B. / TU Berlin. 1991. - Untersuchungen und Kartierung von Waldschäden mit Methoden der Fernerkundung Abschlußbericht (B6) für das BMFT Verbundprojekt

Kenneweg und Liesebach 1984 Kenneweg, H. ; Liesebach, M.: Luftbildauswertung und die Analyse großräumiger Inventurdaten zur Walderkrankung. In: Der Forst- und Holzwirt, Hannover 39 (1984), S. 142-150

Kim 1988 KIM, C.: Signaturanalyse auf der Grundlage von CIR-Luftbildern zur quantitativen Erfassung des Kronenzustandes der mitteleuropäischen Baumarten, Univ. Freiburg, Dissertation, 1988

Kleman 1986 KLEMAN, J.: The spectral reflectance of stands of Norway spruce and Scotch pine, measured form a helicopter. In: Remote Sensing of Environment 20 (1986), S. 253-256

Kleman 1987 KLEMAN, J.: Directional reflectance factor distributions for two forest canopies. In: Remote Sensing of Environment 23 (1987), S. 83-96

Koch 1987 KосH, B.: Untersuchungen zur Reflexion von Waldbäumen mit unterschiedlichen Schadsymptomen auf der Grundlage von Labor- und Geländemessungen, Forstliche Fakultät, Universität München, Dissertation, 1987

Koch et al. 2002 Koch, B. ; Svoboda, J. ; Adler, P. ; Dees M.: Automatische Baumartenerkennung auf der Grundlage digitalisierter CIR-Luftbilder. In: Allgemeine Forst- und Jagdzeitung 173 (2002), Nr. 7-8, S. 131-140

Konecny und Lehmann 1984 Konecny, G. ; Lehmann, G.: Photogrammetrie. 4. Berlin : de Gruyter, 1984 
Kraus 1990 KRAUS, K.: Fernerkundung. Band 2: Auswertung photographischer und digitaler Bilder. Bonn : Dümmler, 1990

Kraus 1996 KRAUS, K.: Photogrammetrie. Band 2: Verfeinerte Methoden und Anwendungen. Bonn : Dümmler, 1996

Kraus 1997 Kraus, K.: Photogrammetrie. Bandl: Grundlagen und Standardverfahren. Bonn : Dümmler, 1997

Landauer und Voß 1989 LANDAUER, G. ; Voss, H.-H. ; LANDAUER, G. (Hrsg.) ; Voss, H.-H. (Hrsg.): Untersuchung und Kartierung von Waldschäden mit Methoden der Fernerkundung: Abschlußdokumentation. Oberpfaffenhofen : DLR, 1989

Leckie et al. 1992 LeCKiE, D. ; YuAn, X. ; OstafF, D.P. ; Piene, H. ; MACLEAn, D. A.: Analysis of high resolution multispectral MEIS imagery for spruce budworm damage assessment on a single tree basis. In: Photogrammetric Engineering \& Remote Sensing 40 (1992), S. 125-136

Levesque und King 2003 LeVesque, J. ; KING, D.J.: Spatial analysis of radiometric fractions from high-resolution multispectral imagery for modelling individual tree crown and forest canopy structure and health. In: Remote Sensing of Environment 84 (2003), S. 589-602

Lillesand und Kiefer 1994 LILlESAND, T.M. ; KIEFER, R.W.: Remote Sensing and Image Interpretation. 3. Aufl. New York : Wiley, 1994

Lindeberg 1994 LindeBERG, T.: Scale-Space Theory in Computer Vison. Dordrecht, Nederlands : Kluwer, 1994 (The Kluwer International Series in Engineering and Computer Science)

Magnussen und Boudewyn 1998 Magnussen, S. ; Boudewyn, P.: Derivations of stand heights from airborne laser scanner data with canopy-based quantile estimators. In: Canadian Journal of Forest Research 28 (1998), Nr. 7, S. 1016-1031

Mansberger 1992 MANSBERGER, R.: Ein System zur visuell-digitalen Zustandsbeurteilung von Baumkronen auf Farb-Infrarot-Luftbildern. Wien, Universität für Bodenkultur, Wien, Fachgruppe Forst- und Holzwirtschaft: 97, Dissertation, 1992

Mather 1999 MATHER, P.: Computer processing of remotely-sensed images: an introduction. Chichester : Wiley, 1999

Mauser 1998 MAUSER, H.: Die Verwendung des Luftbildes bei Inventuren im Schutzwald mit besonderer Berücksichtigung photogrammetrischer Messungen an Einzelbäumen zur Beurteilung von Entwicklungsphasen, Universtät für Bodenkultur,Wien, Bd. 47, Dissertation, 1998 
Maxin 1991 MAXIN, J.: Untersuchung zum Anwendungspotential der analytischen Photogrammetrie für die Dauerbeobachtung im Luftbild, Universität Freiburg, Dissertation, 1991

Mayer 1958 MAYER, R.: Kronengröße und Zuwachsleistung der Traubeneiche auf süddeutschen Standorten. In: Allgemeine Forst- und Jagdzeitung 129 (1958), S. 151163

Mendenhall et al. 1990 Mendenhall, W. ; WACKERLY, D.D. ; SCHAEFFER, R.L.: Mathematical Statistics with Applications. 4th ed. PWS-KENT, 1990

Millard und Nagaraj 2001 Millard, S.P. ; NAgARAJ, K.N.: Environmental Statistics with S-Plus. 2. Aufl. CRC Press, 2001 (Applied Environmental Statistics)

Miller et al. 2000 MilleR, D.R. ; QUine, C.P. ; HADley, W.: An investigation of potential of digital photogrammetry to provide measurements of forest characteristics and biotic damage. In: Forest Ecology and Management 135 (2000), S. 279-288

Mitasova und Hofierka 1993 Mitasova, H. ; HofierKa, J.: Interpolation by Regularized Sline with Tension: II Application to terrain modeling and surface geometry analysis. In: Mathematical Geometry 25 (1993), S. 657-669

Montgomery et al. 2001 Montgomery, D.C. ; PeCK, E.A. ; Vining, G.G.: Introduction to Linear Regression Analysis. 3. Aufl. New York : Wiley, 2001

Mozgawa 1984 MozgaWA, J.: Photogrammetrischer Nachweis von Veränderungen der Kronenformen in immissionsgeschädigten Fichtenbeständen. In: AFZ (1984), S. 774-776

Münch 1995 MÜNCH, D.: Bestandesdynamik von Naturwaldreserven - eine Dauerbeobachtung in Luftbildzeitreihen, Univ. Freiburg, Dissertation, 1995

Murtha 1972 MurThA, P.A.: A guide to air-photo Interpretation of forest damage in Canada. 1972 (1292). - Canadian Forestry Publication

Naesset 1997 NAESSET, E.: Determination of mean tree height of forest stands using airborne laser scanner data. In: ISPRS Journal of Photogrammetry \& Remote Sensing 52 (1997), S. 49-56

Neteler 2000 Neteler, M. ; Mosimann, T. (Hrsg.) ; Kuhnt, G. (Hrsg.): GRASS-Handbuch: Der praktische Leitfaden zun Geographischen Informationssystem GRASS. Abteilung Physische Geographie und Landschaftsökologie am Geographischen Institut der Universität Hannover : Abteilung Physische Geographie, 2000 (Geosynthesis 11)

Neteler und Mitasova 2002 Neteler, M. ; Mitasova, H.: Open source GIS: a grass GIS approach. Boston : Kluwer, 2002 (Kluwer international series in engineering aned computer science) 
Oester 1991 OESTER, B.: Erfassen der Waldschaden-Entwicklung anhand von grossmaßstäblichen Infrarot-Farbluftbildern, ETH Zürich, Vol. 19, 1991

OSI 2002 OSI: Open Source Definition. 2002. - URL http://www.opensource.org/docs/definition_plain.php

Paparoditis und Polidori 2002 Paparoditis, N. ; POlidori, L.: Digital Photogrammetry. Kap. Overview of digital surface models, S. 159-164. New York : Taylor \& Francis, 2002

PCI Geomatics 2001 PCI Geomatics: Airphoto DEM Component. PCI Geomatics, Ontario, Canada. 18 April 2001

PostgreSQL Global Development Group 2002 PostgreSQL Global DeveLOPMENT GROUP: PostgreSQL Homepage. http://www.postgresql.org/. 2002. URL http: //www. postgresql.org/

Pretzsch 2002 PRETZSCH, H.: Grundlagen der Waldwachstumsforschung. Berlin : Parey, 2002

Pröbsting 1996 PRÖBSTING, T.: Luftbildgestützte Verfahren für die Forstwirtschaft: Bedarf, Anwendungsbereiche, Auswertungssysteme, Univ. Göttingen, Dissertation, 1996

Prodan 1965 ProdAn, M.: Holzmesslehre. Sauerländer, 1965

R Project 2002 R Project: What is R? 2002. - URL http://www.r-project.org/

Rabenau und Blasbichler 1969 RABEnAU, G. ; Blasbichler, D.: Hilfsmittel zur Holzartenerkennung im Luftbild. In: Allgemeine Forstzeitung (Österreich) 80 (1969), Nr. 6, S. 132-133

Reid 1992 REID, N.J.: Remote sensing and forest damage. In: Environmental science and technology series 21 (1992), S. 428-429

Richards und Xiuping 1999 RICHARDS, J. A. ; XIUPING, J.: Remote sensing digital image analysis: an introduction. Berlin : Springer, 1999

Riggs und Running 1991 Riggs, G.A. ; Running, S.W.: Detection of canopy water stress in conifers using the airborne imaging spectrometer. In: Remote Sensing of Environment 35 (1991), S. 51-68

Rock et al. 1988 Rock, B. ; Hoshizaki, T. ; Miller, J.R.: Comparison of In Situ and airborne spectral measurement of the blue shift associated with forest damage. In: Remote Sensing of Environment 24 (1988), S. 109-127 
Roerdink und Meijster 2001 RoERdink, J. ; MEIJster, A.: The Watershed Transform: Definitions, Algorithms and Parallelization Strategies. In: Fundamenta Informaticae 41 (2001), S. 187-228

Roloff 1989 RoLOFF, A.: Kronenentwicklung und Vitalitätsbeurteilung ausgewählter Baumarten der gemäßigten Breiten. Frankfurt a. M. : Sauerländer, 1989 (Schriften aus der Forstlichen Fakultät der Universität Göttingen und der Niedersächsischen Versuchsanstalt 93)

Sader und Winne 1992 SADER, S.A. ; WINNE, J.C.: RGB-NDVI colour composites for visualizing forest change dynamics. In: International Journal of Remote Sensing 13 (1992), Nr. 16, S. 3055-3067

Sayn-Wittgenstein 1960 SAYN-WitTGEnSTEIn, L.: Recognition of tree species on air photographs by crown characteristics. Comp. Dept. For. Techn. Note 95.1960

Schardt et al. 2002 Schardt, M. ; Ziegler, M. ; Wimmer, A. ; WACK, R. ; HYYPPÄ, J.: Assessment of Forest Parameters by Means of Laser Scanning. In: Symposium 2002, September 9-13, 2002 Graz. Austria ISPRS Commision III, WG III/3 (Veranst.), 2002, S. A-302ff

Scheer 2000 ScheER, L.: Multi-image manipulation of digital image data for increasing the distinctions between some forest characteristics. In: ZIHALAVNIK, S. (Hrsg.) ; SCHEER, L. (Hrsg.): III. International Symposium: Application of Remote Sensing in Forestry, Preceedings Zvolen, September 12-14 Technical University in Zvolen, Faculty of Forestry (Veranst.), 2000

Scherrer et al. 1994 Scherrer, H. U. ; Schmidtke, H. ; Oester, B.: Folgeaufnahmen: Erfassen von Veränderungen des Waldzustandes mit Luftbildern. 1994 (338). - Eidgenössische Berichte der Eidgenössischen Forschungsanstalt für Wald, Schnee und Landschaft

Schneider 1989 SchneIDER, W.: Verfahren, Möglichkeiten und Grenzen der Fernerkundung für die Inventur des Waldzustandes. In: FBVA-Berichte, Schriftenreihe der Forstlichen Bundesversuchsanstalt, Sonderheft (1989)

Schowengerdt 1997 SCHOWENGERDT, R. A.: Remote sensing, models and methods for image processing. San Diego : Academic Press, 1997

Schramm et al. 1989 SCHRAMm, M. ; GutTMAnN, S. ; JANOTH, J. ; LAMBERTY, M. ; ZWENGER, M.: Abschlußdokumentation: Untersuchung und Kartierung von Waldschäden mit Methoden der Fernerkundung. Teil A. Kap. Großflächige Diagnose und Erfassung von Waldschädne mit Hilfe von Multispektral-Scanner-Daten für das Gebiet desFrankfurter Stadtwaldes, S. 213-232. Oberpfaffenhofen : Mory+Meier, 1989 
Schröck 1994 SCHRÖCK, H.W.: Kronenzustand auf Dauerbeobachtungsflächen in Rheinland-Pfalz-Entwicklung und Einflußfaktoren. 1994 (Mitteilungen aus der Forstlichen Versuchsanstalt Rheinland)

SEMEFOR 2002 SEMEFOR ; EUROPEAN COMISSION (Hrsg.): SEMEFOR: Satellite Based Environmental Monitoring of European Forest. Office of Official Publications of the European Communities, 2002. - Project report, Contract ENV4CT97-0398

Softmap 1999 SOFTMAP, Inc.: BC2000/DSR2000/P100 Upgrade Kit. Columbus, Ohio. 1999

Spanner et al. 1990 SPAnNer, M.A. ; PierCe, L.L. ; Peterson, D.L. ; Running, S.W.: Remote sensing of temperate coniferous forest leaf area index. The influence of canopy closure, understory vegetation and background reflectance. In: International Journal of Remote Sensing 11 (1990), S. 95-111

Spellmann 1984 Spellmann, H.: Dendrometrische Luftbildauswertungen in Kiefernbeständen für Zwecke der Forsteinrichtung. In: Forstarchiv 57 (1984), S. 20-24

Spiecker 1991 SPIECKER, H.: Zur Steuerung des Dickenwachstums und der Astreinigung von Trauben- und Stieleichen (Quercus petrea (Matt.) Liebl. Und Quercus robur L.). Bd. 72. Schriftenreihe der Landesforstverwaltung Baden-Württemberg, 1991

Spurr 1960 SPURR, S.H.: Photogrammetry and Photo-Interpretation. 2. Aufl. New York : Ronald Press Company, 1960

Stellingwerf und Hussin 1997 Stellingwerf, D.A. ; Hussin, Y.A.: Measurements and Estimations of Forest Stand Parameters Using Remote Sensing. 1. Aufl. Utrecht : VSP, 1997

Sysavath et al. 2001 SYSAVATH, V. ; MEnZ, J. ; MARTIENSSEN, T.: Waldmonitoring unter besonderer Berücksichtigung der aus den Stereoluftbildern abgeleiteten 3. Dimension. In: SEYFERT, E. (Hrsg.): 22. Wissenschaftliche-Technische Jahrestagung der DGPF, 24.-26.09.2002, Neubrandenburg Bd. 11, 2001, S. 133-142

Tschupke 1974 TSCHUPKE, W.: Untersuchungen zur automatischen Identifizierung forstlich bedeutsamer Bildgestalten durch digitale Auswertung von an InfrarotFarbluftbildern gemessenen Farb- und Texturparametern, Universität Freiburg, Dissertation, 1974

Tucker 1979 TUCKER, C.J.: Red and photographic infrared linear combinations for monitoring vegetation. In: Remote Sensing of Environment 8 (1979), S. 127-150

Tucker 1980 TuCKER, C.J.: Remote Sensing of leaf watercontent in the near infrared. In: Remote Sensing of Environment 10 (1980), S. 23-32 
Van Gool et al. 1985 Van Gool, L. ; Dewaele, P. ; Oosterlinck, A.: Texture Analysis Anno 1983. In: Computer Vision, Graphics and Image Processing 29 (1985), S. 336-357

Venables und Ripley 2000 VEnABles, W.N. ; RIPLEY, B.D.: S Programming. New York : Springer, 2000 (Statistics and Computing)

Voretzsch et al. 1986 Voretzsch, A. ; Herms, P. ; Hartmann, G.: Standardisierung von CIR-Luftbildern. In: Der Forst- und Holzwirt (1986), S. 420-426

Wang et al. 2002 WAng, Le ; Gong, P. ; Biging, G.S.: Automated individual tree crown delineation and treetop detection in high-spatial resolution aerial imagery. (2002). - URL http: //www. cobblestoneconcepts.com/ucgis 2 summer2002/wang/wang.htm

Westman und Price 1988 Westman, W.E. ; Price, C.V.: Spectral changes in conifers subjected to air pollution and water stress. Experiment studies. In: IEEE Transactions of Geoscience and Remote Sensing (1988), S. 11-21

WGRSA 2000 WGRSA ; Working Group on Remote SENSING ApPlicAtiONS FOR Forest HeAlth Assessment (Hrsg.): Remote Sensing Applications for Forest Health Status Assessment. 2. Aufl. Luxembourg : European Union Scheme on the Protection of Forests against Atmospheric pollution European Commission Office for Official Publications of the European Communities, 2000

Wolf 1970 Wolf, G.: Die Interpetation falschfarbiger Luftbilder im Dienst der Rauchschadensdiagnose von Nadelholzbestockungen. In: Beiträge für die Forstwirtschaft 16 (1970), Nr. 8, S. 18-26

Wolf und Dewitt 2000 WOLF, P.R. ; DEWITT, B.A.: Elements of photogrammetry: with applications in GIS. 3. Aufl. Boston : McGraw-Hill, 2000

Wolff 1992 WolfF, B.: Betriebs- und bestandesweise Holzvorratsinventur auf der Basis von permanenten terrestrischen und Luftbild-Stichproben : am Beispiel des Forstamtes Seesen, Univ. Göttingen, Dissertation, 1992

Wolff et al. 1999 WOLFF, B. ; HABERMAnN, R. ; ARENDT, K.: Evaluierung des Luftbildeinsatzes im Rahmen des deutschen Level II-Monitorings / Bundesforschungsanstalt für Holzwirtschaft. Eberswalde, 1999 (Nr. 96.60DL.020.0). - Abschlußbericht zum EU-Projekt

Wood 1996 Wood, J.: The Geomorphological Characterisation of Digital Elevation Models, Dissertation, 1996. - URL http: //www.soi.city.ac.uk/jwo/phd/

Yuan et al. 1991 YuAn, X. ; KInG, D. ; VlCEK, J.: Sugar maple decline assessment based on spectral and textural analysis of multispectral aerial videography. In: Remote Sensing of Environment 37 (1991), S. 47-54 
Ziegler et al. 2000 ZiEgler, M. ; SChardT, M. ; KonRAD, H.: Einsatzmöglichkeiten von Laserscannerdaten für die Forstinventur. In: FORESTRY, Department of Forest Management and Geodesy Faculty of (Hrsg.): Applications of Remote Sensing in Forestry, Proceedings, Zvolen, September 12-14 2000, 2000, S. 57-64 


\section{Lebenslauf}

\section{PERSÖNLICHE DATEN}

Name: $\quad$ Hans-Jörg Fuchs

Geburtstag: 11.12.1961

Geburtsort: Osterode am Harz

AUSBILDUNG

1974 - 1981 Ernst-Moritz-Arndt-Gymnasium, Herzberg am Harz Abitur

1981 - 1988 Studium der Forstwissenschaft, Albert-Ludwigs-Universität, Freiburg im Breisgau Diplom-Forstwirt

1988 - 1992 Aufbaustudium "Forstwirtschaft in den Tropen und Subtropen", Georg-August-Universität, Göttingen

M.Sc. forest. trop.

\section{BERUFLICHE TÄTIGKEIT}

1989 - 1992 Technischer Angestellter, Institut für Forsteinrichtung und Ertragskunde, Universität Göttingen

1992 - 1994 Vorbereitungsdienst für die Laufbahn des höheren Forstdienstes in Nordrhein-Westfalen Assessor des Forstdienstes

1995 - 1996 Wissenschaftlicher Angestellter, Niedersächsische Forstliche Versuchsanstalt, Göttingen

1996 - 1998 Freiberufliche Tätigkeit:

Waldzustandserhebung in Niedersachsen,

Betriebsinventur in niedersächsischen Forstämtern, Lehrbeauftragter für Vermessungskunde an der Fachhochschule Hildesheim/Holzminden, Fachbereich Forstwirtschaft

seit 1998 Wissenschaftlicher Angestellter, Institut für Waldinventur und Waldwachstum, Universität Göttingen 